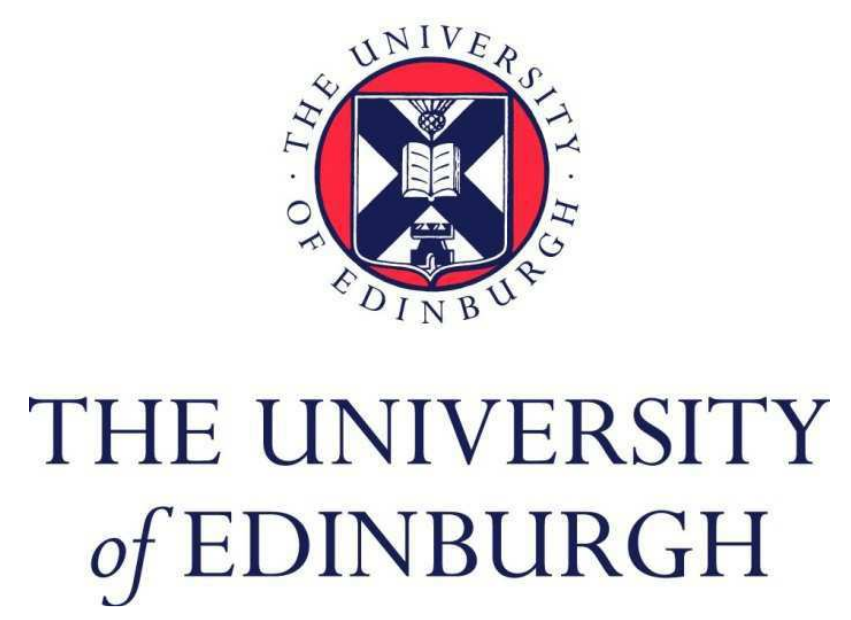

This thesis has been submitted in fulfilment of the requirements for a postgraduate degree (e.g. PhD, MPhil, DClinPsychol) at the University of Edinburgh. Please note the following terms and conditions of use:

This work is protected by copyright and other intellectual property rights, which are retained by the thesis author, unless otherwise stated.

A copy can be downloaded for personal non-commercial research or study, without prior permission or charge.

This thesis cannot be reproduced or quoted extensively from without first obtaining permission in writing from the author.

The content must not be changed in any way or sold commercially in any format or medium without the formal permission of the author.

When referring to this work, full bibliographic details including the author, title, awarding institution and date of the thesis must be given. 


\section{DETECTION OF CARDIAC INFLAMMATION USING ULTRASMALL SUPERPARAMAGNETIC PARTICLES OF IRON OXIDE-ENHANCED MAGNETIC RESONANCE IMAGING}

BY

Colin G Stirrat

A Thesis presented for the degree of Doctor of Philosophy at the University of Edinburgh 


\begin{abstract}
Background Ultrasmall superparamagnetic particles of iron oxide (USPIO)enhanced magnetic resonance imaging (MRI) can detect tissue-resident inflammatory macrophages and identify cellular inflammation. Clinical studies using this non-invasive technique are now emerging.
\end{abstract}

Objectives The aims of this thesis were (i) to determine whether USPIO-enhanced MRI can detect and serially monitor myocardial inflammation after myocardial infarction (MI) using single and repeated USPIO administration, (ii) to report a range of normal R2* (1/T2*) values at 1.5 tesla $(\mathrm{T})$ and $3 \mathrm{~T}$ in healthy myocardium and other tissues before and after USPIO administration, (iii) to determine whether USPIO-enhanced MRI can detect myocardial inflammation in acute myocarditis, and (iv) to determine whether USPIO-enhanced MRI can detect myocardial inflammation in patients with a prior cardiac transplant.

Methods Thirty-one patients were recruited following acute MI and followed up for 3 months with repeated T2 and USPIO-enhanced T2* mapping $3 \mathrm{~T}$ MRI. Twenty healthy volunteers were recruited: 10 imaged each at $1.5 \mathrm{~T}$ and $3 \mathrm{~T}$. T2 and USPIOenhanced T2* mapping MRI was conducted. Fourteen patients with suspected acute myocarditis underwent T2 and USPIO-enhanced T2* mapping 3 T MRI, with further imaging at 3 months. Eleven patients with prior cardiac transplant underwent T2 and USPIO-enhanced $\mathrm{T} 2 *$ mapping $1.5 \mathrm{~T}$ MRI with further imaging at 3 months. Regions of interest within the myocardium, along with other tissues, were selected for analysis. Pre-contrast T2 values, and the change in R2* due to USPIO from baseline to 24 hours after USPIO were compared for each region of interest.

Results In patients with MI, USPIO uptake in the infarct zone peaked at days 2-3, and greater USPIO uptake was detected in the infarct zone compared to remote myocardium in the first 2 weeks after myocardial infarction. In contrast, T2-defined myocardial oedema peaked at days 3-9 and remained increased in the infarct zone throughout the 3-month follow up period. Histology confirmed colocalisation of iron and macrophages within the infarcted, but not the non-infarcted, myocardium.

In healthy volunteers, we reported a range of normal myocardial and tissue R2* values at baseline, and following USPIO. Tissues showing greatest USPIO enhancement were organs of the reticuloendothelial system: the liver, spleen and bone marrow.

Myocarditis was confirmed in 9 of the 14 suspected cases of myocarditis. There was greater myocardial oedema, but no demonstrable difference in USPIO enhancement, in inflamed myocardial regions in patients with myocarditis when compared to healthy myocardium. We recorded an improvement in cardiac function and reduced imaging measures of inflammation after 3 months. 
Ten patients with cardiac transplant were retained for analysis. Measures of myocardial oedema were greater in patients with cardiac transplant than healthy volunteers. There was no difference in the change in R2* due to USPIO between patients with transplantation and healthy volunteers. Imaging recordings did not change when repeated at 3 months.

Conclusions Myocardial macrophage activity can be detected using USPIOenhanced MRI in the first 2 weeks following acute MI. This observed pattern of cellular inflammation is distinct, and provides complementary information to, the more prolonged myocardial oedema detectable using T2 mapping.

In patients with acute myocarditis, USPIO-enhanced MRI does not provide additional clinically relevant information to standard clinical MRI sequences. This suggests that tissue-resident macrophages do not provide a substantial contribution to the myocardial inflammation in this condition.

Stable patients with cardiac transplantation have increased myocardial T2 values, consistent with resting myocardial oedema or fibrosis. In contrast, USPIO-enhanced MRI is normal and stable over time suggesting the absence of chronic macrophagedriven cellular inflammation.

In conclusion, this imaging technique holds promise as a non-invasive method of assessing and monitoring macrophage-driven myocardial inflammation after myocardial infarction with potential application to diagnosis, risk stratification and assessment of novel anti-inflammatory therapeutic interventions. It remains to be determined whether USPIO-enhanced MRI may be able to identify myocardial inflammation in other myocardial inflammatory conditions including acute cardiac transplant rejection. 
For Jill, Lucy and Sophie. 


\author{
Abstract \\ Contents \\ Abbreviations \\ Declaration \\ Acknowledgements
}

CHAPTER 1:

DETECTION OF CARDIAC INFLAMMATION USING ULTRASMALL

SUPERPARMAGNETIC PARTICLES OF IRON OXIDE-ENHANCED

MAGNETIC RESONANCE IMAGING

1.1 Introduction

1.2 Basic principles of cardiac MRI

1.3 Commonly used sequences in cardiac MRI

1.4 T2* imaging in the assessment of iron accumulation

1.5 Iron oxide contrast agents

1.6 Blood pool contrast imaging

1.7 Inflammation imaging

1.8 Summary

1.9 Aims and Hypotheses 
CHAPTER 2:

METHODS

2.1 General

2.2 Magnetic resonance imaging

2.3 Study protocols

$2.4 \quad$ Infusion of USPIO

2.5 Image analysis

2.6 Histological analysis

2.7 Statistical analysis

CHAPTER 3:

FERUMOXYTOL-ENHANCED MAGNETIC RESONANCE IMAGING;

METHODOLOGY AND NORMAL VALUES AT 1.5 TESLA AND 3 TESLA

3.1 Summary

3.2 Introduction

3.3 Methods

3.4 Results

3.5 Discussion

3.6 Study limitations

3.7 Conclusion 
CHAPTER 4:

ULTRASMALL SUPERPARAMAGNETIC PARTICLES OF IRON OXIDEENHANCED T2* MAGNETIC RESONANCE IMAGING CAN BE USED TO DETECT AND SERIALLY MONITOR MYOCARDIAL INFLAMMATION AFTER MYOCARDIAL INFARCTION USING SINGLE AND REPEATED USPIO ADMINISTRATION

4.1 Summary

4.2 Introduction

4.3 Methods

$4.4 \quad$ Results

4.5 Discussion

4.6 Study limitations

4.7 Conclusion

CHAPTER 5:

130-149

FERUMOXYTOL-ENHANCED MAGNETIC RESONANCE IMAGING

IN ACUTE MYOCARDITIS

$5.1 \quad$ Summary

5.2 Introduction

5.3 Methods

$5.4 \quad$ Results

5.5 Discussion

5.6 Study limitations

5.7 Conclusion 
CHAPTER 6:

FERUMOXYTOL-ENHANCED MAGNETIC

RESONANCE IMAGING IN PATIENTS WITH PRIOR

CARDIAC TRANSPLANTATION

6.1 Summary

6.2 Introduction

$6.3 \quad$ Methods

$6.4 \quad$ Results

6.5 Discussion

6.6 Study limitations

6.7 Conclusion

CHAPTER 7:

172-192

CONCLUSION AND FUTURE DIRECTIONS

7.1 Summary

7.2 Summary of thesis findings

7.3 Future directions

7.4 Clinical perspective

REFERENCES

193-207

Publications arising from or relevant to this thesis

208 


\section{ABBREVIATIONS}

\begin{tabular}{|c|c|}
\hline ANOVA & Analysis of variance \\
\hline ANX-SPIO & Annexin V-SPIO \\
\hline $\mathbf{B}_{\mathbf{0}}$ & External magnetic field \\
\hline BMI & Body mass index \\
\hline BSA & Body surface area \\
\hline CABG & Coronary artery bypass graft \\
\hline CD & Cluster of differentiation \\
\hline CI & Confidence interval \\
\hline CKD & Chronic kidney disease \\
\hline CLIO & Cross-linked iron oxide (USPIO) \\
\hline CMR & Cardiac magnetic resonance \\
\hline CRP & C-reactive protein \\
\hline CTIMP & Clinical Trial of an Investigational Medical Product \\
\hline DSI & Delayed signal intensity \\
\hline echos & Echo times \\
\hline EGFP & Enhanced green fluorescent protein \\
\hline ESCs & Embryonic stem cells \\
\hline FDG-PET & ${ }^{18}$ F-Fluorodeoxyglucose positron emission tomography \\
\hline GE & Gradient echo \\
\hline KO mice & Kockout mice \\
\hline LAD & Left anterior descending \\
\hline LGE & Late gadolinium enhancement \\
\hline
\end{tabular}


LV

MHRA

MI

MNPs

MPIO

MRCA

MSCs

MRI

NMV

PCI

PEG

PET

$\mathbf{r}^{2}$

R2*

RES

RF

RITC

ROI

RV

SE

SI

SPIO

SPIO-MAb

SSFP
Left ventricular

Medicines and Healthcare products Regulatory Agency

Myocardial infarction

Magneto-fluorescent nanoparticles

Micron-sized particles of iron oxide

Magnetic resonance coronary angiography

Mesenchymal stem cells

Magnetic resonance imaging

Net magnetisation vector

Percutaneous coronary intervention

Polyethylene glycol

Positron emission tomography

Coefficient of determination

R2-star

Reticuloendothelium system

radiofrequency

Rhodamine B isothiocyanate

Region of interest

Right ventricular

Spin echo

Signal intensity

Small superparmagnetic particles of iron oxide

SPIO-monoclonal antibody

Steady-state free precession 
T helper cell type 1 Th1

$\mathbf{T}$

T1W

T2*

$\mathbf{T} 2 * \mathbf{W}$

T2P

T2W

TE

TI

TR

TrueFISP

USPIO

VSPIO
Tesla

T1-weighted

T2-star

T2*-weighted

T2 prepared

T2-weighted

Echo time

Inversion time

Repetition time

True fast imaging steady-state precession

Ultrasmall superparamagnetic particles of iron oxide

Very small superparamagnetic particles of iron oxide 


\section{DECLARATION}

This thesis represents research undertaken at the Centre for Cardiovascular Sciences, University of Edinburgh and the Edinburgh Heart Centre, New Royal Infirmary of Edinburgh between February 2013 and November 2016.

The studies described in this thesis were funded by the British Heart Foundation (FS/12/83) and the Chief Scientist Office, Scotland (ETM/266). All studies were undertaken in accordance with the Scotland A Research Ethics Committee or the South East Scotland Research Ethics Committee 02, the Medicines and Healthcare products Regulatory Agency and the Declaration of Helsinki of the World Medical Association. Written informed consent of each participant was obtained before entry into the studies.

I was personally involved in every aspect of work presented in this thesis. In keeping with the collaborative nature of research, Dr Shirjel Alam was also involved in the conception of these studies.

This thesis has not been submitted elsewhere in application for a university degree and all sources of information have been acknowledged. Chapters 1, 3, 4, 5 and 6 have either been published in peer-reviewed journals or are currently under review.

\section{Colin Stirrat}

4th October 2017 


\section{ACKNOWLEDGEMENTS}

This thesis has been conducted under the supervision and mentorship of Professor David Newby. Professor Newby guided and encouraged me throughout my research, and testament to his character, his support continues despite leaving his department. I have learned invaluable lessons from him, in particular the qualities he displays as a leader encouraging his team to reach their potential. He always has an 'open door' policy. Professor Newby expects high standards and although he never criticises, every research fellow dreads receiving the notorious 'let's discuss' email reply that greets your inbox soon after sending him a manuscript that fails to meet expectations!

I have worked closely with other people that deserve mention especially at the start of my research where analysis methods were unclear. Doctors Scott Semple, Tom MacGillivray and Calum Gray at the Clinical Research Imaging Centre, University of Edinburgh have vast knowledge and were always on hand to provide support and answer questions. Dr Peter Henriksen was always on hand for advice and to help fine-tune manuscripts.

I would also like to acknowledge the British Heart Foundation and the Chief Scientist Office, Scotland for supporting these studies through research grants. Without their generosity this research material, and indeed this thesis, would not be possible. I am also indebted to the perseverance of Jean Cunningham whose eagleeyed attention to detail has been crucial in the successful formatting of this thesis. 
Most importantly of all, I would like to acknowledge my family. My parents worked hard to ensure I had the opportunity to choose my own path in life. Lastly, I would like to mention my wife Jill, and my two daughters, Lucy and Sophie. Jill has been there for me every step of the way, especially during times when I questioned my future in research and she continues to ensure perspective in my life. I am incredibly grateful to her for her love and support. Alongside Jill, Lucy and Sophie are the apples of my eye and are growing into beautiful, and determined, girls. It is to Jill, Lucy and Sophie that I dedicate this thesis. 


\section{CHAPTER 1}

INTRODUCTION:

DETECTION OF CARDIAC INFLAMMATION USING

ULTRASMALL

SUPERPARAMAGNETIC PARTICLES OF IRON OXIDEENHANCED

MAGNETIC RESONANCE IMAGING 


\subsection{INTRODUCTION}

Beyond the anatomical and morphological information afforded by standard clinical cardiovascular imaging techniques, it is increasingly recognised that additional information examining underlying biological processes would be valuable in a range of cardiovascular diseases to aid understanding of disease pathogenesis, provide diagnostic information, predict disease progression, and to monitor response to treatment. Novel molecular and cellular imaging techniques can identify metabolic and biological processes that are critical to disease pathogenesis, and therefore have the potential to predict clinical events and guide therapeutic intervention. It would therefore be a major clinical advance if a reliable non-invasive imaging technique capable of detecting tissue inflammation could be developed.

A range of potential imaging modalities exists (Table 1.1). Ultrasound is readily available, has good temporal and spatial resolution, is non-invasive, avoids ionising radiation and is relatively cheap. However it suffers from poor penetration and relies heavily on an experienced operator and patient factors such as body habitus and good acoustic windows for optimal imaging. Single-photon emission computerised tomography (SPECT) and positron emission tomography (PET) both suffer from relatively poor spatial and temporal resolution and involve radiation so are not ideal for serial cardiac imaging. Poor penetration of fluorescence imaging limits it's application for inflammatory cardiac imaging in vivo, in man.

Magnetic resonance imaging (MRI) has a number of advantages including excellent discrimination of soft tissue components, good spatial resolution and in particular, 
absence of ionising radiation, an important consideration if serial imaging is envisaged. Here, I will discuss the basic principles of CMR image acquisition, comment upon the use of T2* MRI in the assessment of iron accumulation, and review previous studies employing iron oxide nanoparticles as contrast agents for cardiac MRI. 


\section{TABLE 1.1 Summary of commonly used molecular imaging modalities}

\begin{tabular}{lllllll}
\hline Modality & Spatial & Temporal & Sensitivity & Penetration & Radiation & Cost \\
& Resolution & Resolution & & & \\
& 10-100 micron & High & Low & Good & No & High \\
MRI & 50 micron & High & Medium & cm & No & Medium \\
US & $1-2 \mathrm{~mm}$ & Low & Medium & Good & Yes & Medium \\
PET & $1-2 \mathrm{~mm}$ & Low & High & Good & Yes & High \\
Fluorescence & $2-3 \mathrm{~mm}$ & High & High & $<1 \mathrm{~cm}$ & Y/N & Low
\end{tabular}

MRI - magnetic resonance imaging; US - ultrasound; SPECT - single-photon emission computed tomography; PET - positron emission tomography. 


\subsection{BASIC PRINCIPLES OF CARDIAC MAGNETIC RESONANCE}

\section{IMAGING}

The origin of the magnetic resonance imaging signal is based on nuclear magnetic resonance, which is the response of the hydrogen nuclei to radiofrequency (RF) waves. Each hydrogen nucleus consists of one proton nucleus orbited by one electron. It is the lack of a neutron within the nucleon that allows the hydrogen atom to be magnetically active, giving it a property called 'spin', allowing the proton a small magnetic moment. Although there are other atoms that possess similar magnetic activity, hydrogen is most commonly used for MRI due to its abundance in the human body and large magnetic moment. MRI techniques are essentially based on receiving and processing RF signals from protons.

Protons usually have a randomly orientated magnetic axis. In the presence of a magnetic field however, protons align in the direction, or against the direction of the external magnetic field $\left(\mathrm{B}_{0}\right)$ (Figure 1.1 ). They are unable to align exactly with $\mathrm{B}_{0}$ but rather spin around this axis. Generally, there is a net magnetisation in the direction of $\mathrm{B}_{0}$, called longitudinal magnetisation, although protons possessing more intrinsic energy may align in the opposite direction. In magnets with higher field strength, the native energy possessed by protons is unable to overcome the field strength of the magnet, and greater numbers of protons align in the direction of $\mathrm{B}_{0}$, increasing the net magnetisation vector (NMV). 


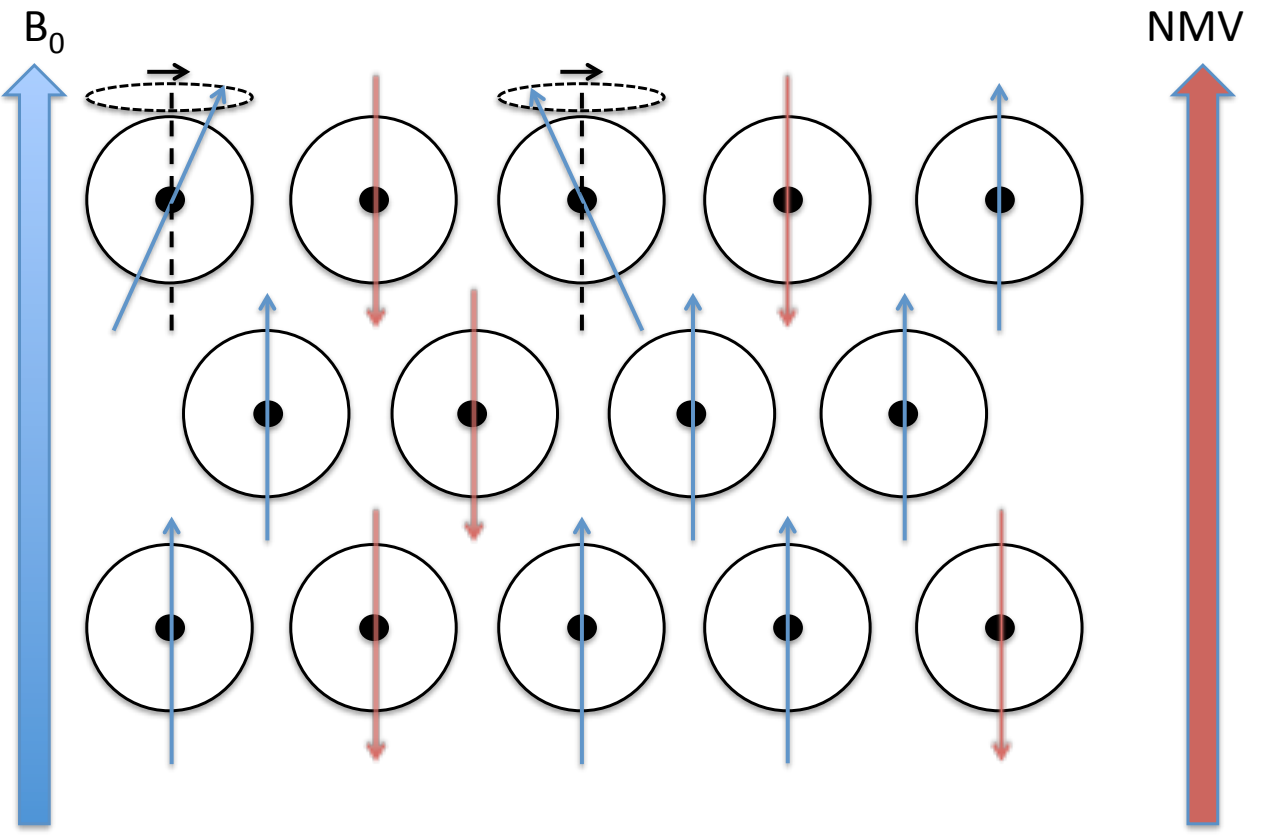

Figure 1.1. In the resting state within an MRI scanner, protons align either in the direction, or in opposition to the external magnetic field, $\mathrm{B}_{0}$. They do not align exactly with $\mathrm{B}_{0}$ but rather spin around an axis that is aligned with $\mathrm{B}_{0}$. An excess of protons align with $\mathrm{B}_{0}$ and the net magnetisation vector (NMV) is in the direction of $\mathrm{B}_{0}$. In the resting state there is no phase coherence as illustrated by the two out-of-phase protons spinning around their axis. MRI - magnetic resonance imaging. 
The spin of protons is termed precession, and the rate at which they spin is termed the precession frequency, which changes linearly as the field strength of the magnet increases. When protons precess in synchrony they are in-phase, and when they are out of synchrony, this is termed out-of-phase.

\subsubsection{RESONANCE AND THE RADIOFREQUENCY PULSE}

Resonance occurs when particles are excited by energy at the same frequency as their own oscillation (the Larmor frequency). For protons precessing at a frequency within a clinical MRI scanner, the frequency of energy required for resonance to occur is within the RF range of the electromagnetic spectrum. The equilibrium of the magnetic field within an MRI scanner can be disrupted by a further magnetic field applied at the Larmor frequency perpendicular to the existing magnetic field. This is termed the radiofrequency field, and the excitation pulse called the RF pulse, transmitted by the RF coil. During excitation, a proportion of protons will gain sufficient energy to flip their axis and spin in the opposite direction from $\mathrm{B}_{0}$. As a consequence of this, the NMV is altered away from its resting state and the angle at which it is altered is termed the flip angle. The flip angle is directly proportional to the amplitude and duration of the RF pulse. In addition, the RF pulse causes all protons, previously precessing out-of-phase, to precess in-phase. This 'phase coherence' enhances the MRI signal generated. 


\subsubsection{DETECTION AND PROCESSING OF THE MAGNETIC RESONANCE SIGNAL}

Fluctuating magnetic fields generated by a radiofrequency pulse in an MRI scanner induce a voltage in the conductive RF coil placed nearby. The RF coil acts as both a signal detector and transmitter, and allows the signal received to be conveyed to specialised computer processors that generate images through a process known as the Fourier transformation. This image formation process is complicated and will not be discussed in depth. Somewhat simplified, by applying a phase encoded magnetic field gradient during the RF pulse, the resonant frequencies of the spins that are subsequently generated vary spatially. As the position of a spinning proton is proportional to its frequency, the Fourier transformation is able to calculate the anatomical position of a signal by determining its spatial frequency.

Following the radiofrequency pulse, protons gradually lose energy, lose phase coherence, and return to their previous state to align with, or opposite to $\mathrm{B}_{0}$. In doing so they recover longitudinal magnetisation ( $\mathrm{T} 1$ recovery) and lose transverse magnetisation (T2 relaxation) simultaneously.

\subsubsection{RELAXOMETRY AND T2*}

$\mathrm{T} 1$ recovery (Figure 1.2) and T2 relaxation (Figure 1.3) are independent exponential processes that are tissue and environment specific. T2 decay is caused by loss of energy between spinning protons that rapidly lose phase coherence gained from the 


\section{T1 Recovery}

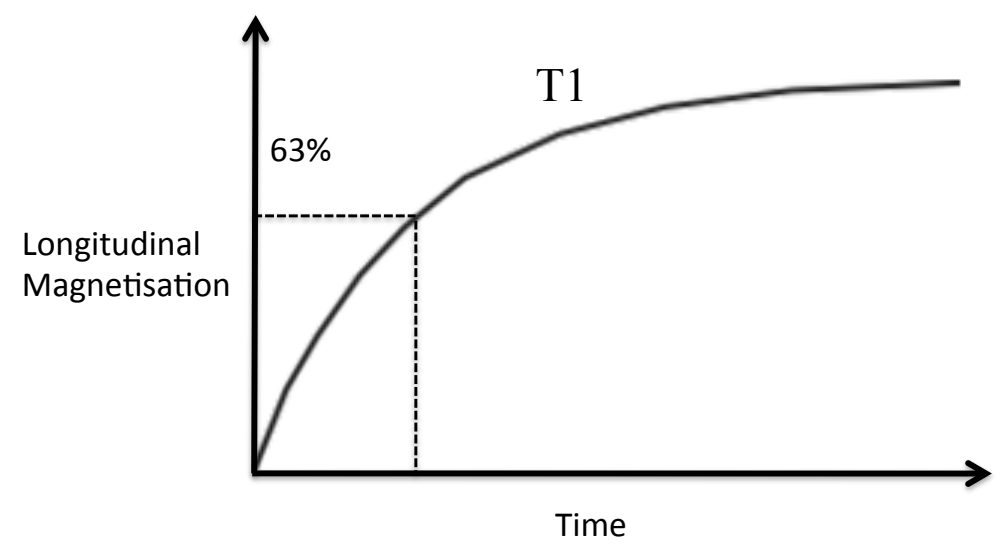

Figure 1.2. T1 recovery occurs during realignment of net longitudinal magnetisation following the $\mathrm{RF}$ pulse. The $\mathrm{T} 1$ value is the time taken for $63 \%$ of longitudinal magnetisation to be recovered. $\mathrm{RF}$ - radiofrequency. 


\section{T2/T2* Decay}

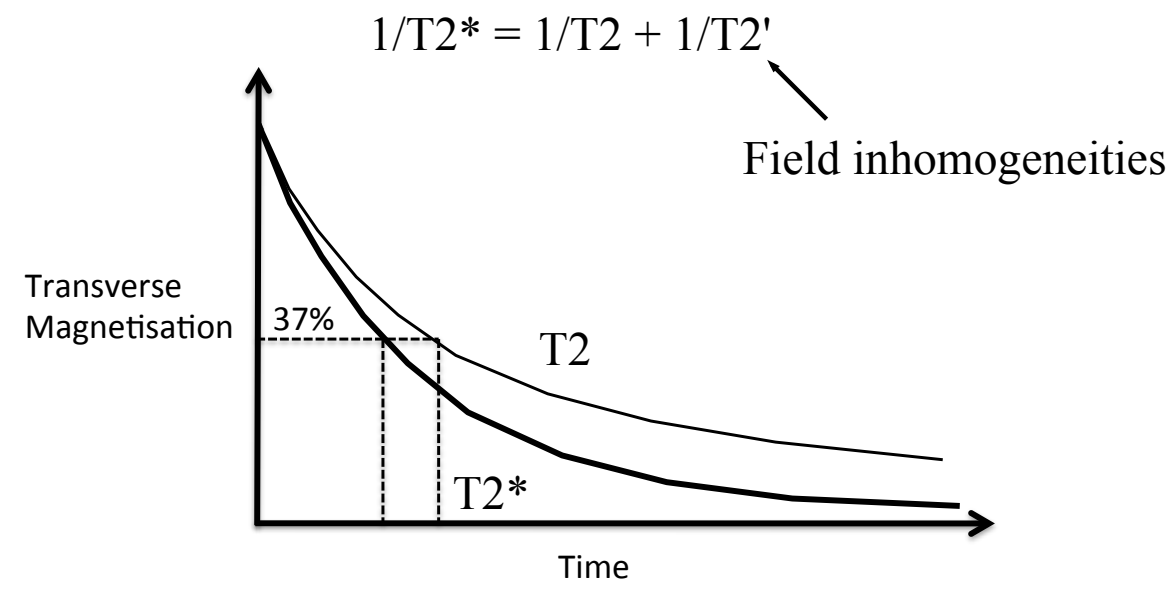

Figure 1.3. T2 decay occurs as a result of spin-spin interaction and dephasing of protons. The $\mathrm{T} 2$ value is time taken for transverse magnetisation to decay to $37 \%$ of its original value. T2* decay is the combination of T2 decay in addition to loss of phase coherence due to magnetic field inhomogeneities. 
RF pulse. This loss of phase coherence occurs prior to realignment of net magnetisation ( $\mathrm{T} 1$ recovery), so transverse relaxation always decays faster than longitudinal recovery. Therefore $\mathrm{T} 2$ is always shorter than $\mathrm{T} 1$. Values can be attributed to $\mathrm{T} 1$ and $\mathrm{T} 2$; the $\mathrm{T} 1$ value is the time taken for $63 \%$ of longitudinal recovery, and $\mathrm{T} 2$ value is the time taken for $\mathrm{T} 2$ to decay to $37 \%$ of the original value.

$\mathrm{T} 2 *$ decay is the combination of $\mathrm{T} 2$ decay in addition to loss of phase coherence due to small magnetic field inhomogeneities. Due to this additional decay process, $\mathrm{T} 2 *$ is always less than T2. These inhomogeneities may be the result of intrinsic defects in the magnet itself, or from susceptibility-induced field distortions produced by tissues or other materials, particularly iron and calcium, placed within the field. Therefore $\mathrm{T} 2 *$ imaging is ideally placed to detect exogenous iron-based contrast agents.

\subsubsection{BASIC PARAMETERS}

Following the radiofrequency pulse, a period of time is allowed to elapse before data sampling takes place. This is called the echo time (TE) and determines the amount of T2 decay that occurs between RF pulses, so a long TE increases the T2 weighting of the sequence. Data sampling at multiple echo times can be made after a single RF pulse to improve accuracy of T2 decay curve fitting. Similarly, the time between RF pulses is called the repetition time (TR) and this determines the amount of $\mathrm{T} 1$ recovery between pulses. Therefore by manipulating TE and TR, the sequence can be optimised for $\mathrm{T} 1, \mathrm{~T} 2$ or $\mathrm{T} 2 *$ depending on the tissue under examination. For example, fat has a short $\mathrm{T} 1$ and $\mathrm{T} 2$, whereas water has a long $\mathrm{T} 1$ and $\mathrm{T} 2$. 


\subsection{COMMONLY USED SEQUENCES IN CARDIAC MAGNETIC RESONANCE IMAGING}

There are two types of sequence fundamental to cardiac MRI; gradient echo (GE) and spin echo (SE). In GE imaging, the composite field strength varies along the axis of $\mathrm{B}_{0}$, being equal to $\mathrm{B}_{0}$ at the centre of the magnet, steadily increasing in strength to one side of $\mathrm{B}_{0}$, and steadily decreasing in strength to the opposite side. As the precession frequency of protons is directly proportional to the magnetic field strength, protons along this magnetic gradient spin at different frequencies. They do not exhibit phase coherence. If a second magnetic gradient in the opposite direction is then applied, phase coherence occurs and the signal sampled at this moment is called the gradient echo. In GE sequences, both blood and fat appear white, so this technique is known as 'white-blood' imaging. Generally gradient echo imaging provides some advantages over spin echo in that flip angles are usually smaller, so recovery of longitudinal magnetisation is faster, allowing shorter repetition times. Rephasing with gradient echo is also faster than the $180^{\circ} \mathrm{RF}$ pulse used in spin echo, and this allows for shorter echo times. As a consequence of shorter TE and TR, scan times are shorter. The disadvantages of GE are poor signal-to-noise ratio and susceptibility to magnetic field inhomogeneities, although this can be advantageous when trying to detect iron. Gradient echo imaging clinically forms the basis of functional imaging and also inversion recovery techniques used in late gadolinium enhancement (LGE) imaging. 
Spin echo imaging uses a different principle, that after the initial RF pulse, a further $180^{\circ}$ pulse is applied to reverse the NMV in the opposite direction, further rephasing protons to achieve phase coherence before data sampling takes place. This additional pulse that rephases protons is required because after the initial RF pulse, spinning protons begin to rapidly dephase and precess at different frequencies, particularly in regions of inhomogeneites. If this did not take place, the signal would be lost before T1 and T2 weighting can be achieved. In spin echo sequences, blood is usually black, fat is white, so is often referred to as 'black-blood' imaging, commonly used for anatomical imaging.

\subsection{T2* IMAGING IN THE ASSESSMENT OF IRON ACCUMULATION}

Gradient echo $\mathrm{T} 2 *$ sequences are highly sensitive to magnetic field inhomogeneities such as susceptibility artefacts due to the presence of iron. In order to maximise T2*weighting, long echo and repetition times are used. Data can be collected at multiple consecutive echo times (echos) following a single RF pulse, speeding up data acquisition, reducing the need for co-registration image that would be required if individual echos were acquired from separate RF pulses.

The intensity of $\mathrm{T} 2 *$ signal exponentially decreases with time and the curve can be plotted using data collected from each echo time. The $\mathrm{T} 2 *$ decay value is the initial decay constant that is calculated using the equation $S(t)=S(0) \exp -\left(t / T 2^{*}\right)$ where $S(t)$ is the signal intensity at time $t$, and $S(0)$ is the signal intensity at time 0 . Thus an increasing rate of decay, as is seen with iron accumulation, results in a reduction in 
$\mathrm{T} 2 *$ value. However iron accumulation is often described and quantified as R2*, the reciprocal of $\mathrm{T}_{2}^{*}$, which has a linear relationship to tissue iron concentration [Wang et al 2005; Wood 2005].

The presence of both endogenous and exogenous iron, including iron-based contrast agents, results in local magnetic field inhomogeneities, rapid dephasing of protons and faster decay of transverse magnetisation. T2* imaging for assessing iron accumulation has successfully been used clinically for over a decade in diagnosing and grading the severity of iron accumulation in transfusion-dependent thalassaemia major, helping guide therapy that improves prognosis, and provides serial disease monitoring [Anderson et al 2001; Anderson et al 2004]. T2* MRI has improved sensitivity in detecting myocardial iron accumulation compared to serum iron sampling [Anderson et al 2001]. This technique is easily quantifiable, well validated, highly reproducible, clinically robust, and is achievable in a single breath-hold [Westwood et al 2003a; Westwood et al 2003b; Tanner et al 2006; Carpenter et al 2009; Kirk et al 2010; Carpenter et al 2011]. Using identical methodology to that described throughout this thesis, Alam et al found excellent repeatability for the assessment of $\mathrm{R} 2 *$ with a mean bias of $-0.208 \mathrm{~s}-1$, coefficient of repeatability of $26.96 \mathrm{~s}^{-1}$, and intraclass coefficient of 0.989 [Alam et al 2012].

T2 also varies inversely with myocardial iron concentration [Wood 2005] although its reciprocal R2 (1/T2) has a curvilinear relationship with tissue iron, in contrast to the more linear relationship of tissue iron and R2* [Anderson et al 2001; 
St Pierre et al 2005], and for this reason, T2* (or R2*) imaging has generally been preferred.

\subsection{IRON OXIDE CONTRAST AGENTS}

Iron oxide particles are a group of magnetic resonance imaging contrast agents that were initially used for gastrointestinal, reticuloendothelial system and lymph node imaging [Saini et al 1987; Hahn et al 1990; Rogers et al 1994], and subsequently as a blood pool contrast agent for the evaluation of cerebral, myocardial and renal perfusion [Canet et al 1993; Trillaud et al 1995; Zimmer et al 1995].

\subsubsection{CLASSIFICATION OF IRON OXIDE PARTICLES}

Iron oxide particles can be categorised according to their size: nano-sized very small (VSPIO, $<20 \mathrm{~nm}$ in diameter), ultrasmall (USPIO, 20-50nm in diameter) and small (SPIO, 60-250nm in diameter) superparamagnetic particles of iron oxide, and micron-sized (MPIO, 1-8 $\mu \mathrm{m}$ in diameter) particles of iron oxide. VSPIO and SPIO are often classified together (as USPIO) due to similar size. Paramagnetism occurs when the size of the iron oxide crystal is smaller than that of ferromagnetic domains (approx. 30nm). Under the influence of a strong external magnetic field, such as exists within an MRI scanner, iron oxide nanoparticles have a large magnetic moment, but unlike larger ferromagnetic particles, this magnetic moment is lost when the field is removed. Table 1.2 lists the different applications for USPIO previously used in MR imaging. 


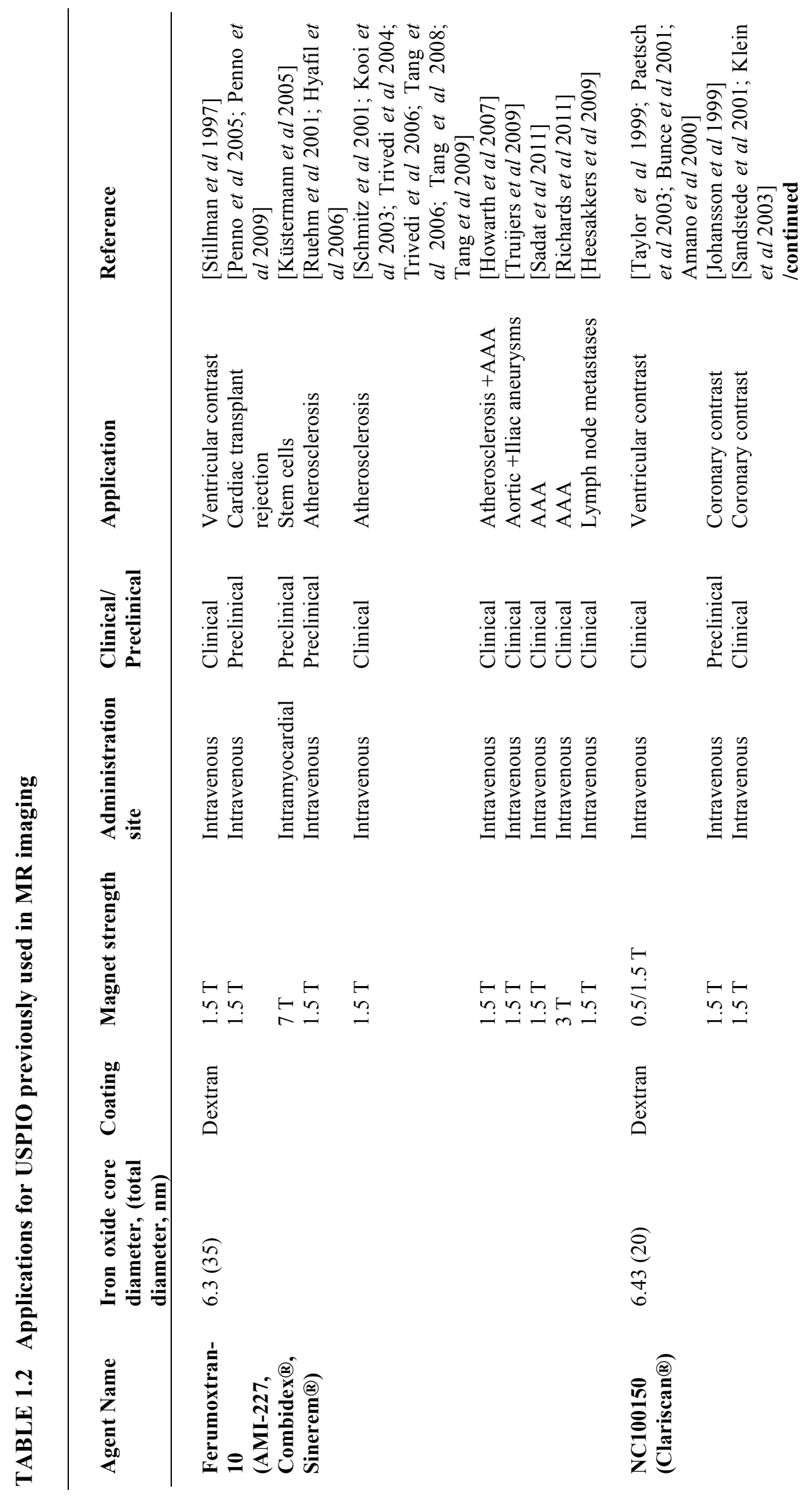




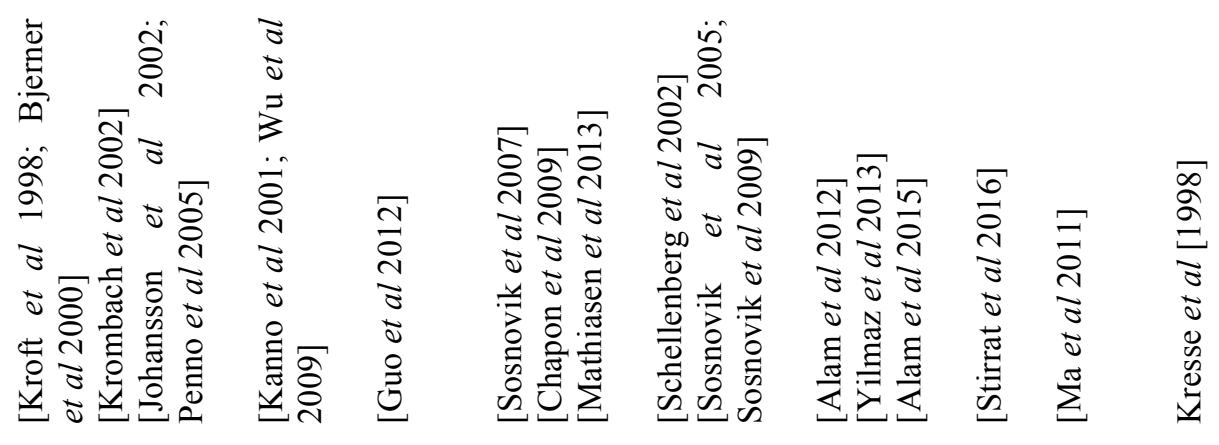

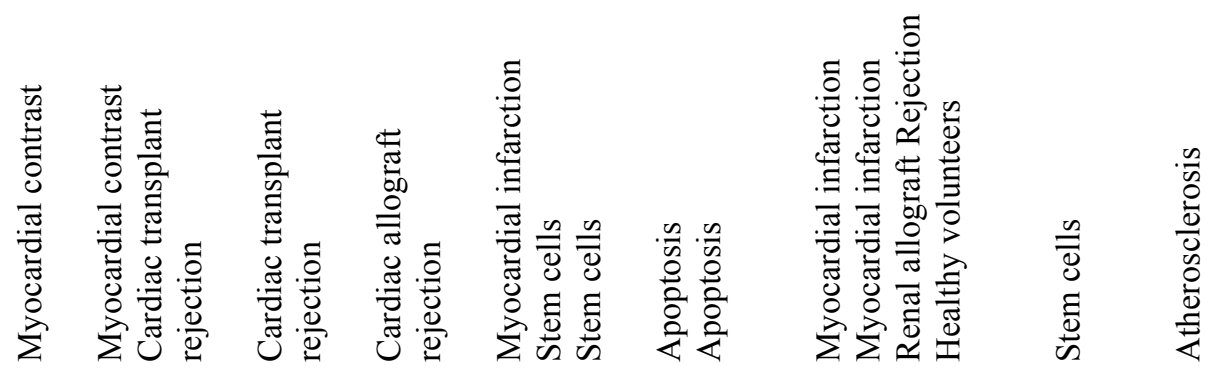

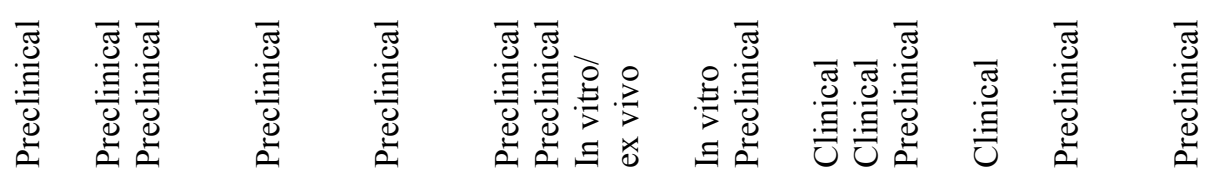

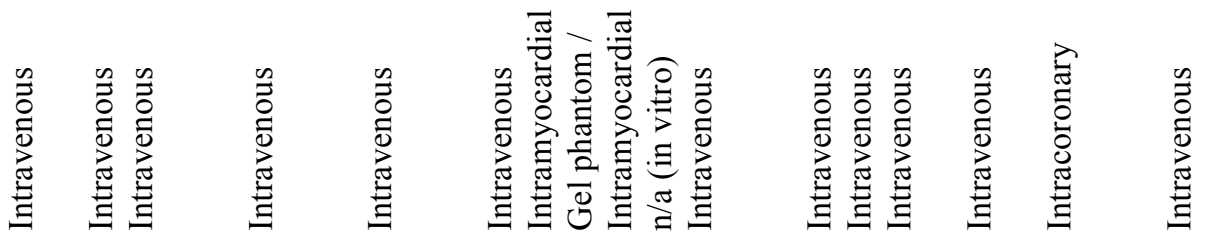

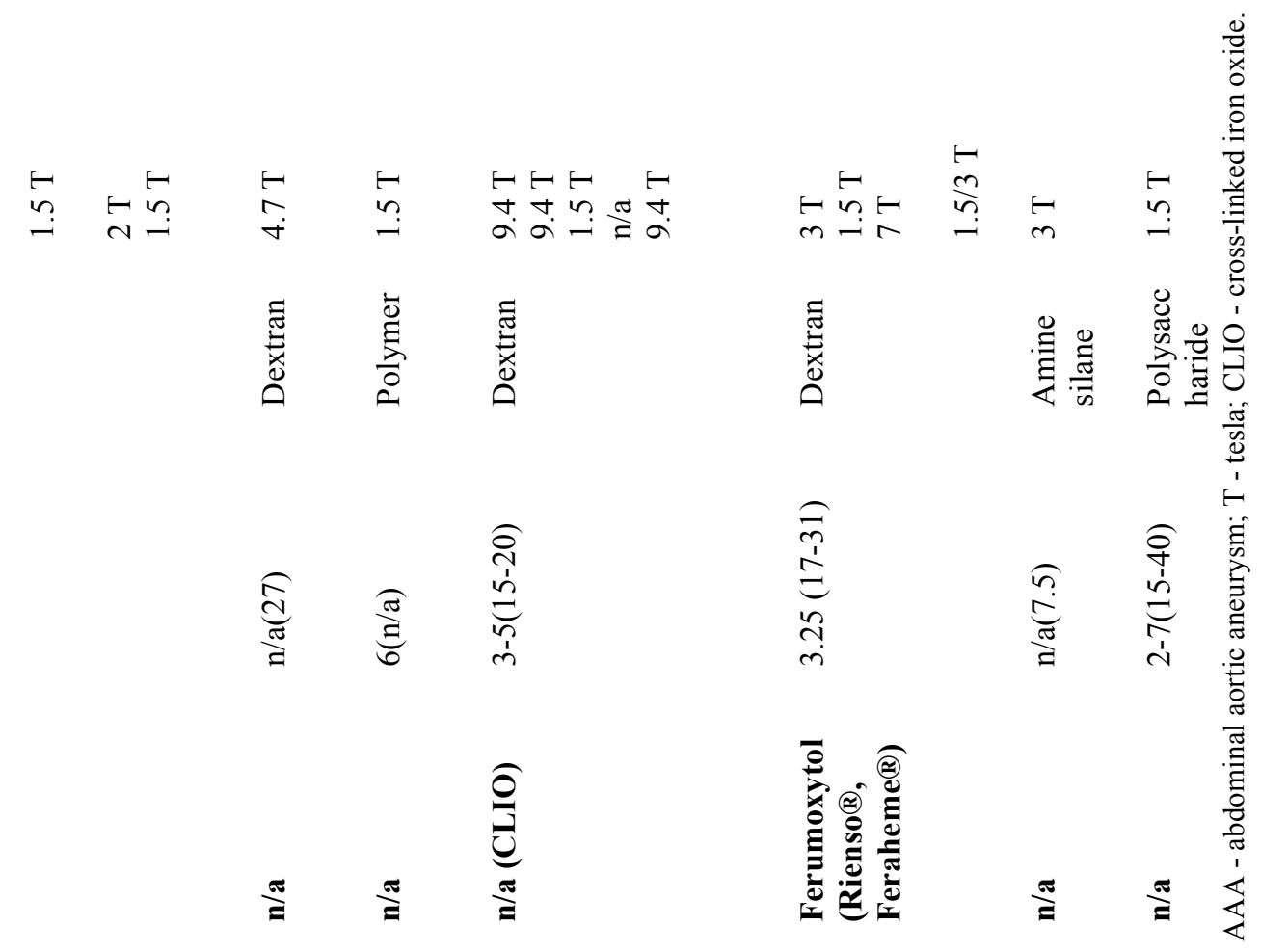




\subsubsection{CONSTRUCTION AND DISTRIBUTION OF IRON NANOPARTICLES}

Ultrasmall superparmagnetic particles of iron oxide generally consist of an iron oxide core surrounded by a coating, usually of carbohydrate or polymer construction, with a total particle diameter of less than $50 \mathrm{~nm}$. The purpose of the coating is to prevent aggregation, to retain particles within a colloidal suspension and to provide a surface for conjugation of drug molecules and targeting ligands. A range of particles exists varying in size and coating; these two properties are the key determinants of biodistribution, magnetic behaviour and thus the potential applications of the particle [Mandeville et al 1997; Allkemper et al 2002]. The biodistribution of USPIO is individual to each particle but in general a blood pool half-life of several hours is expected with distribution predominantly in the spleen, liver, bone marrow and lymph nodes, before being cleared within a matter of days. Cytotoxicity data for iron nanoparticles is limited but is not found until exposure to high levels of iron and is also dependent on factors such as surface coating or it's breakdown products, composition of the cell medium, and protein-iron nanoparticle interaction [Berry et al 2003; Berry et al 2004; Mahmoudi et al 2009; Mahmoudi et al 2010].

\subsubsection{CELLULAR DETECTION OF IRON NANOPARTICLES}

The majority of proposed imaging applications for iron oxide nanoparticles utilise particles within the ultrasmall and small size ranges. Following administration, cells of the reticuloendothelial system, particularly tissue macrophages, identify and internalise iron oxide nanoparticles (Figure 1.4). Ingestion of ultrasmall superparamagnetic particles of iron oxide appear to be predominantly by phagocytes of the reticuloendothelial system, as well as tumour cells, although uptake by 
granulocytes such as neutrophils does occur albeit less readily [Moore et al 1997;

Gellissen et al 1999; Kaim et al 2002; Weinstein et al 2010; Han et al 2015].

Lymphocytes are not phagocytic and therefore do not ingest USPIO.

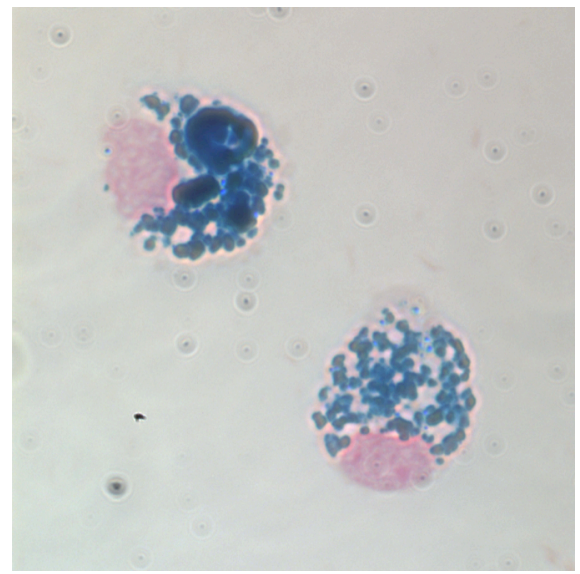

Figure 1.4. Perl's iron stain reveals aggregates of SPIO within the cytoplasm of human mononuclear cells labelled with SPIO ex vivo. SPIO - small superparamagnetic particles of iron oxide. 
Due to their larger size, small superparamagnetic particles of iron oxide are more easily identified and rapidly engulfed, principally by resident macrophages in the lymphoreticular organs of the liver, spleen and lymph nodes. In contrast, USPIO are much smaller and escape immediate phagocytosis, resulting in a much longer blood pool half-life compared to larger particles (up to 36 hours for USPIO [McLachlan et al 1994] versus as low as 2 hours for larger SPIO in humans [Clément et al 1998]).

Whilst circulating within the blood pool, USPIO are small enough to migrate passively across the endothelium [Wang et al 2001] especially in regions of inflamed vasculature where there is loss of endothelial integrity. Once present within tissues, nanoparticles are concentrated and engulfed by resident macrophages through phagocytosis and pinocytosis resulting in their accumulation within inflamed tissues. Concentration and compartmentalisation within macrophages results in magnetic cooperativity and enhances their superparamagnetic effects. This in turn generates markedly shorter $\mathrm{T} 2$ and $\mathrm{T} 2 *$ relaxation times than would be achieved at similar iron concentrations within the interstitium [Brisset et al 2010]. In the presence of iron, local signal deficits are created on T2- and T2*-weighted images, and thus USPIO can be employed as a 'tracer', highlighting areas of tissue inflammation on MRI (Figure 1.5) [Wang 2011]. 

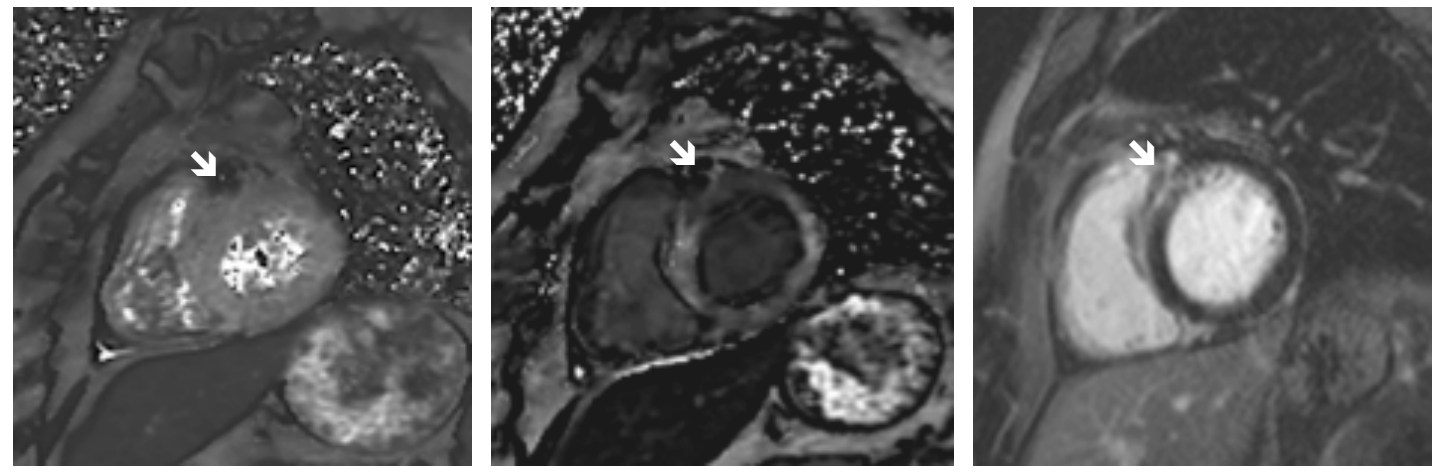

Figure 1.5. Patient imaged 5 days after an anterior myocardial infarct with $\mathrm{T} 2 *$-weighted magnetic resonance imaging. Images show $\mathrm{T} 2 *$ maps of baseline pre iron scan (left), and 24 hours following ferumoxytol (USPIO) administration (middle), and late gadolinium enhancement in the region of infarction (right). Iron uptake is seen in the dark myocardial regions in the region of the anterior infarct (arrow), and also the liver and ventricular cavity blood pool. USPIO - ultrasmall superparamagnetic particles of iron oxide. 


\subsubsection{COMMERCIAL AVAILABILITY AND SAFETY OF IRON NANOPARTICLE AGENTS}

The development of iron nanoparticle-enhanced MRI has been greatly affected by the discontinuation of several agents in recent years. The safety profile of the USPIO, ferumoxtran-10 (Sinerem $\AA)$, was felt to be acceptable $(<1 / 200$ Serious Adverse Event risk) and importantly not toxic to macrophages, but production was discontinued due to a high false-positive rate of detecting lymph node metastasis in patients with prostate cancer [Bourrinet et al 2006; Muller et al 2007; Bernd et al 2009; Heesakkers et al 2009]. NC100500 (Clariscan ${ }^{\mathrm{TM}}$ ) was discontinued over concerns regarding long-term liver retention [Ho and Reddy 2010], and the larger SPIO ferumoxides (Feridex ${ }^{\circledR}$ in USA, Endorem ${ }^{\mathrm{TM}}$ in Europe), and ferucarbotran (Resovist ${ }^{\circledR}$ ) have also been discontinued; the latter two agents were the only two clinically approved SPIO agents for MRI. At present there is only one USPIO preparation available for clinical use, and this is in the USA only: ferumoxytol (Feraheme $^{\mathrm{TM}}$, AMAG Pharmaceuticals), a $17-30 \mathrm{~nm}$ USPIO. Feraheme ${ }^{\mathrm{TM}}$ is marketed for the intravenous treatment of iron deficiency anaemia in adult patients with chronic kidney disease (CKD), but is currently not licensed for MR imaging. The manufacturer recently terminated its EU marketing license (branded Rienso ${ }^{\circledR}$ in the EU), due to economical and not safety reasons. Serious hypersensitivity reactions with ferumoxytol are rare $(0.2 \%)$ and anaphylaxis has been recorded, but no such episodes have been observed in several hundred intravenous injections at our centre [Richards et al 2011; Alam et al 2012; McBride et al 2015; Stirrat et al 2016 and 2017]. 


\subsubsection{ANALYSIS TECHNIQUES AND TECHNICAL CONSIDERATIONS}

Earlier studies using iron nanoparticles employed semi-quantitative methods to calculate reduction in signal intensity consistent with iron accumulation. Such methods involve the comparison of relative signal intensities of a study tissue to a control tissue (such as healthy skeletal muscle). However these methods are liable to variations in signal across the image relative to proximity to surface coils between visits, and also assumes that signal intensity in the control tissue is consistent across scans. Calculation of changes in $\mathrm{T} 2$ and $\mathrm{T} 2 *$ relaxivity values has now been adopted for a more sensitive and objective quantitative approach. The $\mathrm{T} 2 *$ relaxivity value is the initial decay constants for the exponential decay of signal intensity with increasing echo time. Calculation and comparison of decay constants for each region of interest allows a more objective and quantitative approach and accounts for the problems mentioned by older techniques. In the presence of iron, the signal decays more rapidly and the $\mathrm{T} 2$ and $\mathrm{T} 2 *$ relaxivity values are reduced. On the resulting images, particularly on $\mathrm{T} 2 *$ imaging that is more sensitive than $\mathrm{T} 2$ imaging for iron accumulation, a profound reduction in signal intensity is evident as a region of signal void (hypointense region) where USPIO have accumulated (see Figure 1.5).

In the following sections, I will review the use of iron oxide nanoparticles in cardiac MRI. I will discuss their previously studied applications as a blood pool contrast agent, and more recently, a tracer highlighting inflammatory tissue macrophages within the heart. 


\subsubsection{VENTRICULAR CONTRAST}

Ultrasmall superparamagnetic particles of iron oxide have been used successfully as coronary and ventricular MRI blood pool contrast agents for around two decades. USPIO have a longer blood pool circulating time compared to standard MRI angiographic agents such as gadolinium. In 1997, Stillman et al demonstrated ferumoxtran-10 administration improved myocardial to blood pool border definition on T1-weighted cine images. Image intensity gradients at the myocardial to blood pool interface increased significantly in both long and short axis views. At the time it was thought that ferumoxtran-10 would improve and facilitate automated ventricular cavity edge detection allowing for easier and less timeconsuming functional analysis.

These findings were largely replicated by Taylor et al [1999] who used an alternative USPIO agent, NC100150 (Clariscan ${ }^{\mathrm{TM}}$ ) with a blood pool half-life of over 2 hours, to again improve ventricular cavity edge detection using MRI. They administered NC100150 to 18 healthy male volunteers and examined cine imaging sequences. At higher doses ( 3 and $4 \mathrm{mg} \mathrm{Fe} / \mathrm{kg}$ ) an increase in signal intensity was seen in the blood pool once again enabling better detection of ventricular blood pool to myocardial interface, improving functional assessment of the left ventricle. These early studies have since been replicated in other human studies (Figure 1.6) [Amano et al 2000; Bunce et al 2001; Paetsch et al 2003]. 

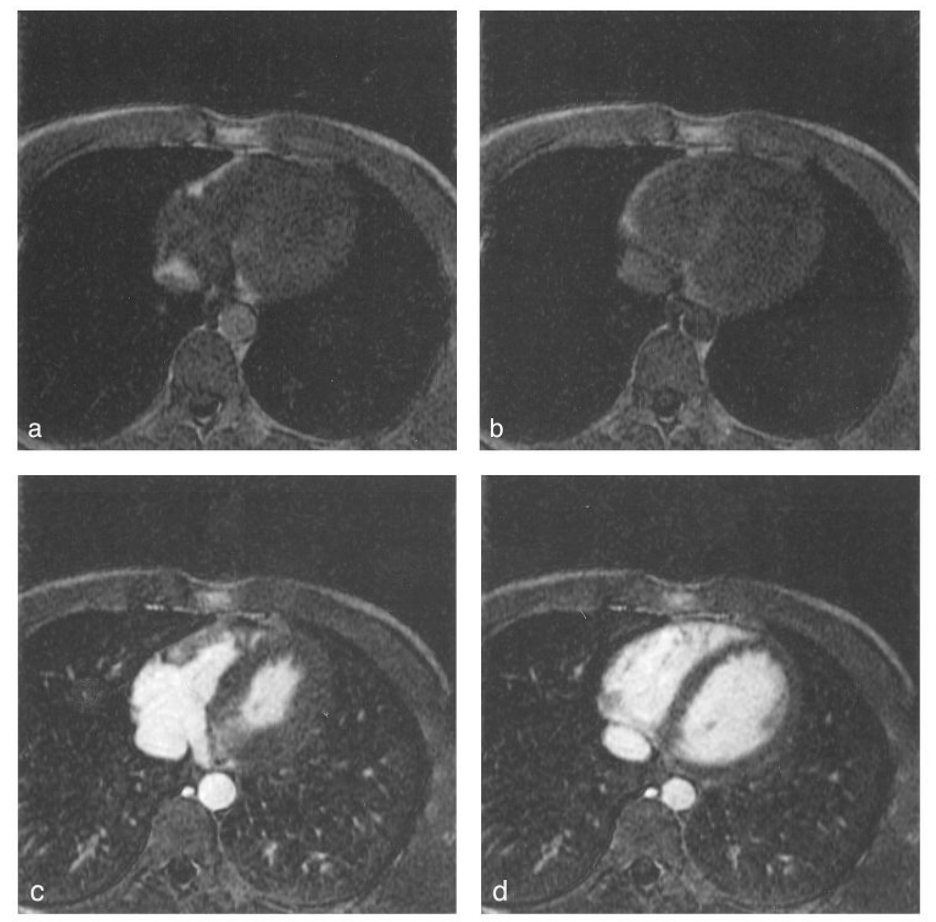

Figure 1.6. A 28-year-old volunteer. A) Pre contrast 3D cardiac cine MR image at end-systole. B) Pre contrast 3D cine image at end-diastole. C) Post contrast 3D cine image at end-systole. D) Post contrast 3D cine image at end-diastole. Pre contrast 3D cardiac cine MR images do not show the contrast between myocardium and ventricular cavity clearly (A and B), whereas post contrast 3D cardiac cine MR images exhibit high imaging contrast between them after administration of USPIO (NC100150) at $3 \mathrm{mg} \mathrm{Fe} / \mathrm{kg}$ body weight (C and D). Reprinted with permission from Amano et al. J Magn Reson Imaging 2000;11:81-86. 


\subsubsection{CORONARY CONTRAST}

Following use as a ventricular blood pool contrast agent, the next logical step worth exploring was the potential for iron nanoparticles to improve coronary artery imaging. This would have an immediate advantage over standard coronary catheterisation, being non-invasive and without radiation. It therefore comes as no surprise that $\mathrm{NC} 100150$ has been used extensively as a coronary contrast agent for magnetic resonance coronary angiography (MRCA), but with mixed results. Johansson et al compared invasive x-ray angiography with T1-weighted MRCA using NC100150 in pigs with coronary artery stenosis [Johansson et al 1999]. Initial results were encouraging. Although the sample size was small $(n=6)$, the location and severity grading of stenoses on NC100150-enhanced MRCA correlated well with x-ray angiography. Within a few years, Sandstede et al [2001] carried out a feasibility study using the same contrast agent, this time in human patients with coronary artery disease. They found that proximal segments of coronary arteries were well visualised, however distal segment visualisation was poor. Increasing doses of USPIO were used in a failed attempt to improve image quality, and only 6 of 8 important proximal coronary stenoses seen on conventional angiography were detected using NC100150 contrast-enhanced MRI.

Klein et al [2003] conducted more coronary imaging comparing NC100150enhanced MRI with unenhanced MRI again yielding disappointing results. Although the visible length of mid and distal portions of the coronary artery improved with USPIO-enhanced imaging, there was no improvement when assessing the proximal segment of the artery. Sensitivity was suboptimal as only 8 of 11 significant coronary 
stenoses ( $>50 \%$ luminal narrowing on conventional angiography) were detected on USPIO-enhanced MRI compared to 6 of 11 on the non-contrast scan.

\subsubsection{MYOCARDIAL VIABILITY}

Soon after the trialling of USPIO as a blood pool contrast agent for ventricular cavity and coronary imaging, attempts were made to illustrate myocardial viability after myocardial infarction (MI). Studies examined the theory that USPIO would illuminate vasculature in viable, but not non-viable, myocardium. Kroft et al [1998] administered ferumoxtran-10 (AMI-227, Sinerem $\left.{ }^{\circledR}\right)$ in a pig model of myocardial infarction. Contrary to expectations, MRI using ferumoxtran-10 failed to illuminate viable myocardium. This was likely due to low dose iron $(30 \mu \mathrm{mol} \mathrm{Fe} / \mathrm{kg})$, fast elimination time of the contrast agent and a low field strength MR scanner (1.5 tesla $(\mathrm{T})$ ). Increasing the magnetic field strength provides improved signal-tonoise ratio, greater spatial resolution and improves sensitivity for detecting lower concentrations of iron, which is advantageous in the detection of USPIO.

Using a similar model, Bjerner et al [2000] had more success using increased iron dosing. Using T1-, T2- and T2*-weighted imaging, they found that T1-weighted MRI at USPIO doses of 4 and $8 \mathrm{mg} \mathrm{Fe} / \mathrm{kg}$ provided the best evaluation of perfused myocardium when compared with fluorescein stain on ex vivo histology.

The use of USPIO as a myocardial tissue contrast agent was extended to a model of ischaemia-reperfusion in rats to evaluate microvascular injury in reperfused myocardium. Krombach et al [2002] employed coronary ligation for 45 minutes and 
waited 3 hours following ligation release to mimic ischaemia-reperfusion, before administering NC100150 to help define regions of microvascular injury and obstruction after myocardial infarction. USPIO distributes by diffusion and is retained in the extravascular space of injured myocardium with damaged, leaky vasculature. They found that USPIO-enhanced imaging provided some indication of transmural and non-transmural microvascular injury. This imaging approach tended to overestimate the area of true infarction when compared to triphenyltetrazolium chloride histological staining, and underestimate the area of myocardium deemed 'at risk' of infarction determined by redo coronary ligation and intravenous phthalocyanine blue dye injection.

\subsection{INFLAMMATION IMAGING}

Endomyocardial biopsy is the 'gold standard' diagnostic investigation for inflammatory diseases of the myocardium, however this carries the risk of life-threatening complications [Cooper et al 2007]. Furthermore, sampling error is common due to the focal nature and spatial heterogeneity of underlying inflammatory processes. Today this procedure is rarely performed outside specialist tertiary referral centres. The following sections discuss applications of iron nanoparticle-enhanced CMR to highlight tissue inflammation as cells of the reticuloendothelial system, particularly tissue macrophages, identify and internalise iron oxide nanoparticles after administration (Figure 1.7). 


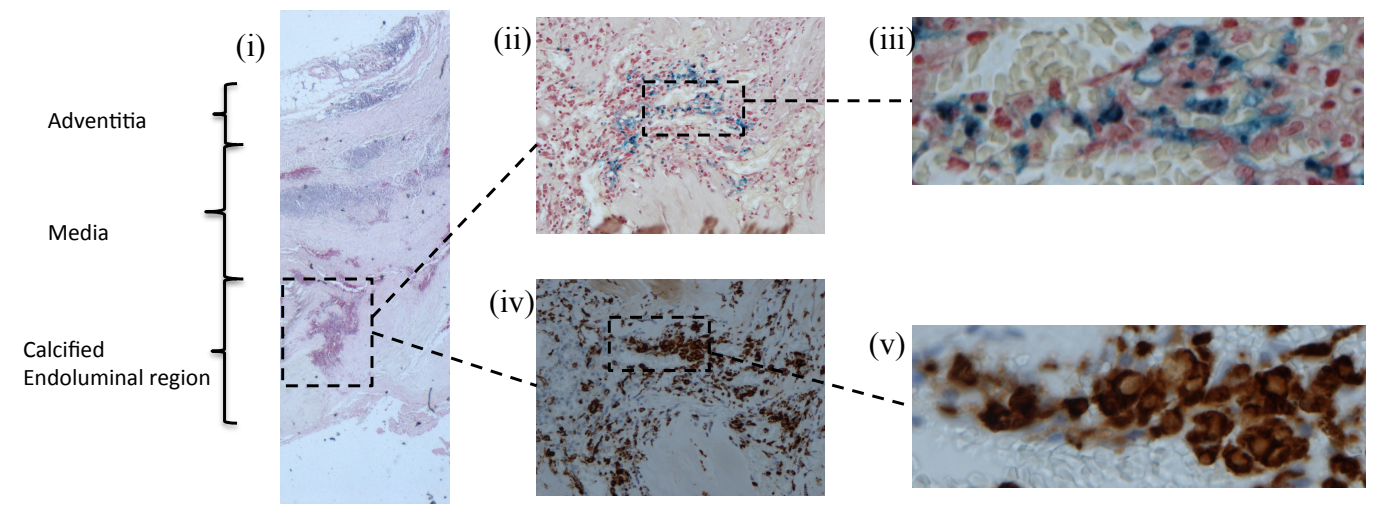

Figure 1.7. Histology from an abdominal aortic aneurysm wall. (i) Haematoxylin and eosin staining at x20 (magnification) - inflammatory cell infiltrates seen in the endoluminal region with focal calcified atheroma. (ii) Perls stain for iron at x100 and (iii) x600 showing the presence of iron (blue). (iv) CD68 staining for macrophages (brown) at $\mathrm{x} 100$ and (v) $\mathrm{x} 600$ showing colocalisation of macrophages and iron staining. CD - cluster of differentiation. 
The development of cross-linked USPIO (CLIO) [Wunderbaldinger et al 2002] opened avenues allowing for more targeted inflammatory imaging. Cross-linking and amination of the USPIO dextran chains encourages circumferential adherence of the dextran coat to the iron nanoparticle providing chemical stability and improved conjugation to other molecules including antibodies thus enabling targeted imaging of specific antigens [Wunderbaldinger et al 2002]. Preclinical studies discussed in the following sections often make use of CLIO for targeted inflammatory imaging.

\subsubsection{MYOCARDIAL INFARCTION}

Myocardial infarction caused by sustained interruption to coronary blood flow is associated with a potent inflammatory response that is essential for cardiac repair. The magnitude of the inflammatory response is greater if ischaemic tissue is reperfused, which is the current standard practice for treating patients with MI in the developed world. The inflammatory response seen is a prerequisite for scar formation and ultimately healing of the injured myocardium.

Acute myocyte death rapidly activates innate immune pathways triggering an inflammatory reaction. Injured, necrotic tissue generates and releases endogenous signals, known as 'alarmins' that promote activation of innate immune cells [Chan et al 2012]. Other intracellular constituents released by necrotic cells also activate an immune response [Arslan et al 2011]. Innate immune cells identify danger signals via engagement of Toll Like Receptors (TLRs) that activate pro-inflammatory cascades. 
Activation of the complement system and generation of reactive oxygen species after ischaemia are also involved in attracting of inflammatory cells. Upregulation of proinflammatory chemokines and cytokines ultimately lead to attraction of inflammatory cells into the heart.

The cellular response is dominated initially by neutrophil influx, and later with interleukin and monocyte-derived macrophage invasion [Entman and Smith 1994; Frangogiannis et al 1998; Frangogiannis et al 2002]. Macrophages contribute a source of cytokines and growth factors necessary to support neovascularisation, extracellular matrix metabolism and fibroblast proliferation. However it is not simply a case of the more macrophages the better. Recently, it has been shown that monocytes and their derived macrophages influence both the extent of myocardial salvage and recovery of left ventricular (LV) function, but if early levels of macrophages become uncontrolled, this leads to poorer outcomes [Maekawa et al 2002; Nahrendorf et al 2007; Tsujioka et al 2009; Nahrendorf et al 2010; Panizzi et al 2010]. Therefore it appears that an 'optimum' degree of macrophage infiltration exists after myocardial infarction, and it would be a major advance if a reliable imaging technique capable of detecting and quantifying inflammatory tissue macrophages after MI could be developed.

Yang et al [2010] successfully imaged inflammatory cell infiltration in a murine left anterior descending (LAD) coronary artery ligation model of myocardial infarction. Large micron-sized particles of iron oxide were administered and $\mathrm{T} 2 *$ signal attenuation was seen in the infarct zone of mice compared with control mice that 
were (i) administered MPIO and had a 'sham' operation, or (ii) subjected to myocardial infarction but did not receive MPIO. Unsurprisingly, they noted increased signal attenuation in the infarct area, illustrating MPIO accumulation secondary to macrophage ingestion, and this was confirmed using histology data. Signal attenuation from accumulation of macrophages correlated with a reduction in left ventricular ejection fraction suggesting greater levels of inflammation predict poorer functional recovery. This adds further evidence to suggest that optimal post-MI recovery is determined by the balance between proinflammatory and reparative macrophages, with uncontrolled early inflammation leading to adverse functional recovery.

The same group subsequently carried out a slightly modified experiment proving that monocytes travelled from storage within organs of the reticuloendothelium system to the myocardium after MI. They injected mice with MPIO and waited 7 days before carrying out iatrogenic myocardial infarction again using LAD ligation. As micronsized particles of iron oxide are rapidly phagocytosed and cleared from the blood within one minute, this allows adequate time for MPIO to be distributed only within organs of the reticuloendothelium system. After LAD ligation 7 days later, mice were imaged at various intervals over the following month, and signal attenuation was seen within the infarct zone for 2 weeks post-MI. The signal attenuation seen must be due to accumulation of MPIO-labelled macrophages originating from the reticuloendothelial system. Myocardial haemorrhage was discounted as a contributing factor to the infarct related signal attenuation as the control group of 
MI-induced mice that did not receive MPIO showed less signal attenuation than the MPIO groups [Yang et al 2011].

Sosnovik et al [2007] used multimodality imaging in assessing inflammation post-MI. They conjugated CLIO with cyanine 5.5 dye creating a magneto-fluorescent particle allowing co-registration of MRI and fluorescence molecular tomography data. They administered CLIO-Cy5.5 48 hours after left coronary artery ligation in a mouse model of myocardial infarction with dual modality imaging 48 hours later. Accumulation of CLIO-Cy5.5 within infarcted regions of myocardium was clearly seen on fluorescence and MR images that were not present on control mice (mice injected with CLIO-Cy5.5 but had a sham operation). In vivo imaging findings were subsequently confirmed with histology: inflammatory cell infiltrate seen on haematoxylin and eosin staining colocalised with regions of increased signal intensity on fluorescence microscopy, and regions displaying an abundance of macrophages on immunohistochemistry staining. This is consistent with uptake of CLIO-Cy5.5 by macrophages infiltrating the infarcted myocardium.

Clinical applications of USPIO highlighting tissue inflammation after myocardial infarction have since emerged. Alam et al [2012] used USPIO to image myocardial tissue inflammation following acute MI. Within 48 hours of acute MI patients underwent MRI scanning at baseline (pre USPIO), before receiving an infusion of ferumoxytol (USPIO) with subsequent serial MRI scans thereafter. Following USPIO administration, there was a significant increase in $\mathrm{R} 2 *(\mathrm{R} 2 *=1 / \mathrm{T} 2 *)$ in the infarct zone (defined by late gadolinium enhancement). Further smaller changes 
were observed in the peri-infarct (surrounding areas of delayed enhancement) and remote myocardial zones when compared with control tissue (skeletal muscle). The authors suggested the R2* increase seen in non-infarcted myocardium was due to pan-myocardial inflammation, however no histological data or healthy volunteer comparison group was available to confirm this. Importantly, changes in R2* were not due to myocardial infarction alone. A control group of MI patients not administered USPIO did not show a change in infarct zone R2*. Notably, the R2* value was greatest 24 hours following USPIO infusion, and had started to decline by 48 hours suggesting that 24 hour post infusion was the most sensitive imaging time point for detecting infarct-related inflammation in this group of patients.

Yilmaz et al [2013] conducted a study of similar design, yielding similar results, detecting significant USPIO uptake within the infarct, peri-infarct and remote myocardium besides the lymphoreticular system. They imaged the first 3 patients at 6, 24, 48 and 96 hours after ferumoxytol administration in an attempt to find the optimal time window to characterise USPIO uptake within the myocardium. Like Alam et al [2012], signal attenuation was maximal at 24 hours, although the authors chose imaging at 48 hours as they considered this the best time for combination imaging of $\mathrm{T} 2$ - and $\mathrm{T} 2 *$ imaging.

The finding of $\mathrm{T} 2 *$ signal void in remote myocardium in both these studies is interesting and may suggest a pan-myocardial inflammatory response in the context of a focal infarct. Clinical histological data are clearly required to address the exact distribution and relationship between USPIO and phagocytosing inflammatory cells 
within the myocardium after MI, and the relative contribution to changes in $\mathrm{T}_{2} *$ of extracellular USPIO accumulation in the interstitium, (peri-) capillary bed and inflammatory macrophages.

\subsubsection{MYOCARDITIS}

Myocarditis is a disease characterised by inflammatory cell infiltration of the myocardium with evidence of myocyte necrosis. Acute myocarditis comprises a wide clinical spectrum from subclinical disease to severe heart failure. There is a variety of different causes for myocarditis that include infections, iatrogenic toxins (such as anthracyclines) and immune-mediated, although frequently no cause is found [Caforio et al 2013].

A variety of inflammatory cells infiltrate the myocardium during myocarditis. The pathogenesis and recruitment of inflammatory cells into the myocardium are determined by the aetiology. In viral myocarditis, the most common cause of myocarditis, myocytes can be injured by direct viral entry and toxicity which can result in cell death and lysis, cytokine expression and inflammatory cell attraction [Esfandiarei $\mathrm{m}$ et al 2008]. In viral myocarditis, the infiltrate is predominantly lymphocyte rich, but plasma cells, neutrophils, eosinophils, giant cells, and macrophages have been found [Aretz et al 1987; Magnani and Dec 2006; Barin et al 2012; Sagar et al 2012; Jaquenod De Giusti et al 2015]. In man, coxsackie B virus is the commonest cause of viral myocarditis [Feldman et al 2000; Li et al 2009]. 
Autoimmune mechanisms have also been implicated in the pathogenesis of myocarditis. Again, viral infection may be the initial insult releasing intracellular antigens that are then recognised as foreign by the body's own antibodies. The T cell response to these 'self-antigens' stimulate other inflammatory B and T cells and cytotoxic cytokines. Autoantibodies to several cardiomyocyte components have been described \{Caforio et al 1997, Schultheiss HP 1989]. Hypersensitivity myocarditis, a further cause for autoimmune myocarditis, is usually related to a recently initiated medication and is characterised by a predominantly eosinophilic infiltrate [Fenoglio et al 1981].

Although not the most prevalent cell type, macrophages appear to be intimately involved in myocarditis. In a rat model of myocarditis, monocytes have been found to differentiate into macrophages at sites of inflammation and exhibit a $\mathrm{T}$ helper cell type 1 (Th1) cytokine/chemokine profile suggesting they play an important role in the pathogenesis of myocarditis [Baba et al 2006]. Again macrophages may have divergent functions. In a mouse model of viral myocarditis, macrophage knock out (KO) mice had higher viral titres but paradoxically lower levels of inflammation seen on histology [Jaquenod De Giusti et al 2015]. In another study, mice with depletion of macrophages failed to develop myocardial inflammation [Hirasawa et al 1996]. These findings may tentatively suggest that similar to myocardial infarction, macrophages have both pro and anti-inflammatory effects in myocarditis.

Endomyocardial biopsy is considered the gold standard, and is recommended for diagnosis however is prone to sampling error and rarely clinically justified or 
performed [Caforia et al 2013]. Cardiac MRI plays a key part in diagnosis and shows typical appearances on $\mathrm{T} 2$-weighted and gadolinium contrast imaging according to the Lake Louise Criteria [Friedrich et al 2009], and recent advances in quantitative MRI mapping techniques have recently been shown to improve diagnostic accuracy (Figure 1.8) [Luetkens et al 2015]. 

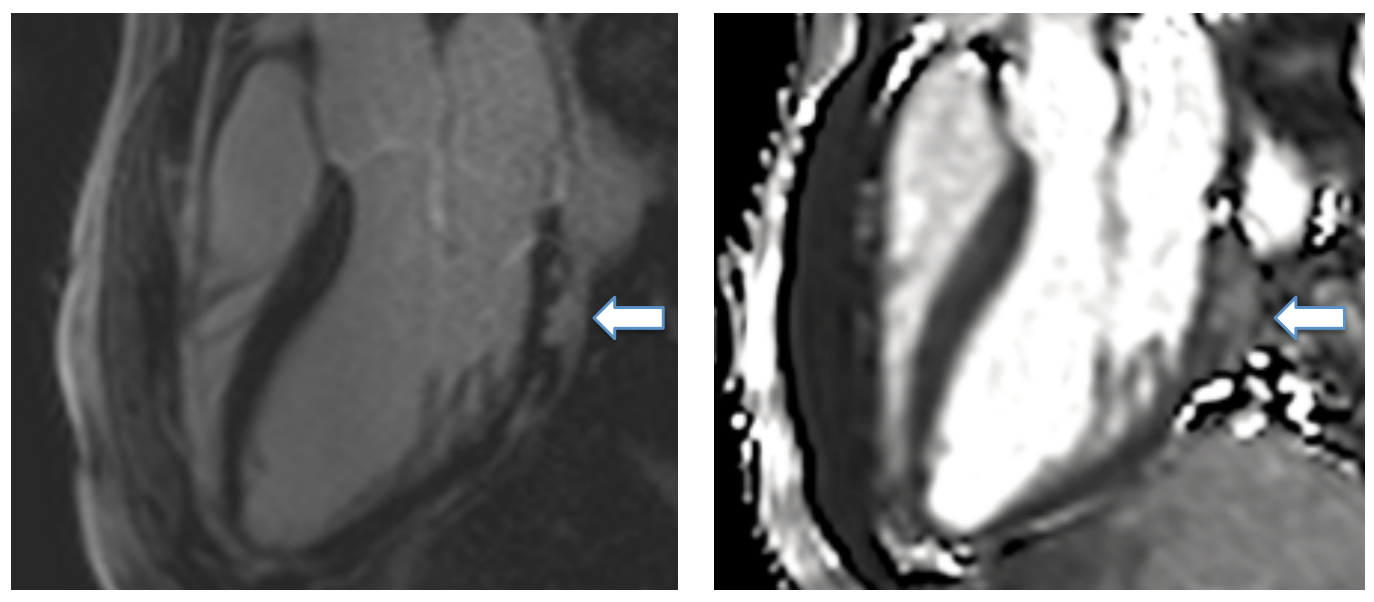

Figure 1.8. Three chamber image of patient with basal posterior wall myocarditis (arrow) displaying typical MRI features of LGE (left) and high signal on T2 mapping (right). MRI - magnetic resonance imaging; LGE - late gadolinium enhancement. 
Experimental models of myocarditis have been scrutinised by molecular imaging employing iron particles. Moon et al [2012] examined a rat model of experimental autoimmune myocarditis. They made use of a dual superparamagnetic and fluorescent 'magneto-fluorescent' nanoparticles (MNPs) consisting of a SPIO core with a rhodamine B isothiocyanate (RITC)-incorporated silica coating of approximately $20 \mathrm{~nm}$ thickness, and polyethylene glycol (PEG) conjugated onto the silica coating. They compared MNP-enhanced (10 mg Fe/kg) MRI with unenhanced conventional MRI protocols using T2-weighted, T1-weighted and early and late gadolinium enhancement sequences. The degree of inflammation was also assessed histologically. MNP-enhanced $\mathrm{T} 2 *$-weighted $\mathrm{CMR}$ more accurately detected scattered foci of inflammation within the myocardium of rats with autoimmune myocarditis and improved visualisation of small and less severe myocardial inflammation than conventional CMR imaging. Areas detected by MNP-enhanced T2*-weighted CMR correlated well with inflammatory foci on histology as well as signal on fluorescence microscopy. However, MNP-enhanced MRI ultimately proved unable to detect milder histological grades of myocardial inflammation, and this may limit its clinical applicability. 


\subsubsection{CARDiaC TRANSPLANTATION}

Cardiac transplantation is a life prolonging treatment option for end stage cardiac disease. Transplant rejection is a major threat to the allograft, occurring in $20-30 \%$ of recipients in the first year [Hertz et al 2009], but can occur at any stage after transplantation and causes significant morbidity and mortality. Rejection is notoriously difficult to diagnose using existing non-invasive imaging methods so surveillance myocardial biopsies are routinely taken after transplantation.

Most cases of acute graft rejection after transplantation are due to cellular rejection with antibody-mediated rejection less prevalent. Acute cell-mediated rejection is primarily a host T-cell response mounted against allograft tissue. Antibody-mediated rejection occurs when the allograft is injured resulting from activation of the complement system, typically by recipient-generated antibodies directed against the allograft tissue.

Following tissue damage by the above mechanisms, bioactive cytokines are released by $\mathrm{T}$ cells and necrotic tissue. Cytokines such as IL-1 $\alpha$ and TNF $\alpha$ are involved in stimulating the production of chemokines that in turn attract neutrophil migration to the site of inflammation [Eigenbrod et al 2008, Ishii et al 2010]. Other 'danger' signaling mechanisms are activated following insult to the graft during the transplantation process. These mechanisms activate dendritic cells leading to antigen-presenting cell maturation and release of proinflammatory cytokines [La Rosa et al 2007, Moreau et al 2013]. Donor antigen presenting cells induce activation and differentiation of alloreactive naïve $\mathrm{T}$ cells into effector $\mathrm{T}$ helper cells 
in secondary lymphoid organs. These effector $\mathrm{T}$ cells subsequently activate macrophages and granulocytes that have infiltrated the graft in response to inflammatory stimuli [Moreau et al 2013].

The severity of rejection is graded according to histological findings, and although the cellular infiltrate in acute cellular rejection is predominantly lymphocytic, macrophage infiltration is known to have a key role [Stewart et al 2005; Mannon 2012]. The importance of macrophages in acute cardiac allograft rejection was highlighted in a recent study that showed depletion of circulating macrophages protected rodent hearts against allograft rejection, thus suggesting potential therapeutic targeting of macrophages in this condition [Wu et al 2013]. This study used USPIO-enhanced MRI to track macrophages within the heart, but the use of this technique in assessing cardiac allograft rejection is not new and has been used for over a decade.

Kanno et al [2001] detected cardiac allograft rejection using MRI and dextran coated USPIO in rats with evidence of allograft rejection, again exploiting the ability of activated macrophages to accumulate iron nanoparticles within their cytoplasm. Histopathology confirmed active rejection and iron staining closely correlated with staining for macrophages. Treatment response could also be monitored with this method; rats that received prolonged immunosuppression, controlling the inflammatory response, show significantly less USPIO uptake on MRI. 
Subsequent studies have again confirmed the value of USPIO-enhanced MRI to detect acute cardiac rejection in rats with intensity of USPIO signal detected correlating with more severe rejection on histology and also poorer cardiac function [Johansson et al 2002; Penno et al 2005; Wu et al 2009; Wu et al 2013], although ferumoxtran-10 proved inferior to NC100150 in one study [Penno et al 2005] and was unable to detect acute rejection in another [Penno et al 2009]. In this latter study, ferumoxtran-10 was given at two doses of $2 \mathrm{mg}$ and $11 \mathrm{mg} \mathrm{Fe} / \mathrm{kg}$ with T2*-weighted MRI conducted using 1.5 T MR imaging at postoperative day 7, 24 hours after USPIO administration. Failure to detect USPIO was likely due to the low magnetic field strength of the MR scanner.

Besides simply tracking tissue macrophages, iron oxide particles can be conjugated to antibodies or peptides allowing targeting of specific pathophysiological processes [McAteer et al 2010]. Recent work by Guo et al [2012] targeted T cells that play a central role in acute cardiac allograft rejection. They used ligand targeting of cells to employ a 'theranostic' strategy, combining diagnostic imaging with administration of therapy within the one system. They targeted $\mathrm{T}$ cells in the endocardium of rejecting rat hearts by using a CD3 antibody multifunctional polymeric nanocarrier that included SPIO and also a therapy gene (pDNA-DGKalpha). This enabled simultaneous imaging by detecting SPIO, at the same time as providing immune modulation to the $\mathrm{CD} 3$ expressing T cells.

USPIO has also proved useful in detecting renal transplant rejection. Recent work by Alam et al [2015] showed that significantly more USPIO accumulated in murine 
renal allografts compared with isografts and native kidneys. USPIO accumulation within macrophages was confirmed using immunohistochemistry and electron microscopy.

\subsection{SUMMARY}

Iron nanoparticles, in particular USPIO, have been used in functional and molecular MR imaging of the heart with variable success over the last 20 years. Initially used as a blood pool contrast agent, it is in their ability to detect cardiac inflammation that there is major promise for clinical translation. This exciting imaging technique has great potential and the results of these research studies, and others in the coming years, will help determine its place in the clinic.

\subsection{AIMS AND HYPOTHESES}

The aim of this thesis is to investigate the role of USPIO-enhanced MR imaging in the detection of cardiac inflammation. Specifically, I will assess whether USPIO-enhanced MRI is able to detect and serially monitor macrophage-driven inflammation after myocardial infarction. Additionally, I wish to assess whether USPIO-enhanced MRI is able to detect cardiac inflammation in other conditions with macrophage infiltration of the myocardium; myocarditis; and cardiac transplant rejection. In order to do this we will also examine a control group of healthy volunteers for comparison. 
The following hypotheses will be addressed:

1. A range of normal $\mathrm{T} 2 *$ values at $1.5 \mathrm{~T}$ and $3 \mathrm{~T}$ in healthy volunteers before and after USPIO administration can be determined (Chapter 3).

2. USPIO-enhanced T2* MRI can be used to detect and serially monitor myocardial inflammation after MI using single and repeated USPIO administration (Chapter 4).

3. USPIO-enhanced T2* MRI can be used to detect myocardial inflammation in acute myocarditis (Chapter 5).

4. USPIO-enhanced T2* MRI can be used to detect myocardial inflammation in cardiac transplantation (Chapter 6). 


\section{CHAPTER 2}

\section{METHODOLOGY}




\subsection{GENERAL}

\subsubsection{ETHICAL AND REGULATORY CONSIDERATIONS}

All studies were approved by the South East Scotland Research Ethics Committee or Scotland A Research Ethics Committee. The Medicines and Healthcare products Regulatory Agency (MHRA) approved the use of Rienso ${ }^{\circledR}$ (Takeda Italia S.p.A. Via Crosa, 86. 28065 Cerano (NO) Italy) to investigate patients with myocarditis, cardiac transplant rejection and healthy volunteers as a Clinical Trial of an Investigational Medical Product (CTIMP). All studies were conducted in accordance with the Declaration of Helsinki. Studies were registered on the public website www.clinicaltrials.gov (NCT01995799; NCT02319278).

\subsubsection{SUBJECT RECRUITMENT}

Written informed consent was received by all participants following appropriate review of the patient information sheet. A copy of the consent form and participant information sheet was filed in the medical case records. Participants' GPs were informed of their inclusion in writing and received a participant information sheet.

\subsubsection{MYOCARDIAL INFARCTION}

Participants were aged 18 to 80 years of age and had sustained a recent MI according to the Third Universal Definition of Myocardial Infarction [Thygesen et al 2012], with 12-hour plasma troponin I concentration $\geq 5000 \mathrm{ng} / \mathrm{L}$. Exclusion criteria were known critical stenosis ( $>95 \%)$ of the left main stem, ongoing symptoms of angina, heart failure (Killip class $\geq 2$ ), renal failure (estimated glomerular filtration rate 
$<30 \mathrm{~mL} / \mathrm{min} / 1.73 \mathrm{~m}^{2}$ ), and contraindication to MR imaging or ferumoxytol infusion.

\subsubsection{Healthy SUBJECTS}

Healthy subjects were recruited via adverts at the Royal Infirmary of Edinburgh, and the Golden Jubilee National Hospital, Clydebank. Healthy subjects were over 18 years of age and had no past medical history of an inflammatory process. Other exclusion criteria were contraindication to MRI or ferumoxytol infusion, pregnancy, breastfeeding and women of childbearing potential without reliable contraception.

\subsubsection{ACUTE MYOCARDITIS}

Adult ( $>18$ years of age) patients with suspected acute myocarditis were recruited into the study. The clinical diagnosis was made by an independent clinical cardiologist based on the history, electrocardiogram, serum troponin and other available imaging modalities. The diagnosis of myocarditis was verified from case note review by an independent cardiologist and retained for analysis if the MRI scan (reported and agreed by two independent radiologists) showed typical imaging features of myocarditis [Friedrich et al 2009]. Healthy volunteers had no clinically significant past medical history. Exclusion criteria were contraindication to MRI or ferumoxytol infusion, any other inflammatory comorbidity, renal failure (estimated glomerular filtration rate $<30 \mathrm{~mL} / \mathrm{min} / 1.73 \mathrm{~m}^{2}$ ), pregnancy, breastfeeding and women of childbearing potential without reliable contraception. 


\subsubsection{CARDIAC TRANSPLANT}

Participants were over 18 years of age and had undergone cardiac transplantation. Exclusion criteria were contraindication to MRI (including pacemaker and ICD) or ferumoxytol infusion, recent myocardial infarction (1 month), any other inflammatory comorbidity, renal failure (estimated glomerular filtration rate $<30 \mathrm{~mL} / \mathrm{min} / 1.73 \mathrm{~m}^{2}$ ), pregnancy, breastfeeding and women of childbearing potential without reliable contraception.

\subsection{MAGNETIC RESONANCE IMAGING}

Basic principles of magnetic resonance imaging are discussed in Chapter 1. Contraindications to MRI scanning included claustrophobia and metallic implants.

\subsubsection{MRI PROTOCOL}

Magnetic resonance imaging was performed using a 3 tesla scanner at the University of Edinburgh and a 1.5 tesla scanner at the Golden Jubilee National Hospital, Clydebank (Magnetom Verio and Avanto respectively, Siemens AG, Healthcare Division GmbH, Erlangen, Germany), all with dedicated cardiac array coils. All images were acquired using electrocardiogram-gated breath-holds.

All cardiac MRI scans had the following sequences as standard. Scanning started with axial and coronal localisers to ensure the heart is in the isocentre of the magnetic field. This was immediately followed by a free breathing, 30 slice series of black-blood HASTE imaging, useful to determine anatomy, followed by breath held 
steady-state free precession (SSFP) vertical and horizontal long, and short axis images of the heart, necessary to plan short axis, 2, 3 and 4 chamber views of the heart.

Following the above standard sequencing, participants were imaged with some or all of three following sequences depending on the specific study protocol.

\subsubsection{T2* IMAGING}

Quantitative USPIO imaging by calculation of $\mathrm{T} 2 *$ decay times was performed in a short axis stack, 2, 3 and chamber views, and a sagittal view through the spine using a prototype $\mathrm{T} 2 *$-weighted multi-echo gradient-echo acquisition using a volumetric shim applied over the entire heart volume (TR 996 ms, TE 2.13, 4.3, 6.4, 8.6, 10.7, 12.8, 15.0, $17.1 \mathrm{~ms}$, flip angle $18^{\circ}$, matrix $130 \times 256$, FoV $400 \mathrm{~mm}$, slice thickness $6 \mathrm{~mm}, 4 \mathrm{~mm}$ gap). The T2*-weighted acquisitions included views through the liver, spleen and spine to allow quantification of USPIO accumulation within organs of the reticuloendothelial system. The same $\mathrm{T}^{*}$ protocol was used to quantify USPIO accumulation 24 hours after infusion allowing calculation of $\mathrm{T} 2 *$ relaxation rates before and after administration of USPIO. The in-plane resolution differed as required for larger or smaller objects; generally, a field of view of $400 \times 300 \mathrm{~mm}$ was used with an in-plane resolution of $2.6 \times 1.6 \mathrm{~mm}$.

\subsubsection{T2 MAPPING}

Oedema imaging was conducted using a Siemens T2 mapping (Siemens Healthcare, Erlangen, Germany) (based on a prototype T2-prepared (T2P) True fast imaging 
steady-state precession (FISP) acquisition acquiring identical long and short axis slice positions (TR $219.3 \mathrm{~ms}$, TE $1.07 \mathrm{~ms}$, T2P durations 0, 25, $50 \mathrm{~ms}$, flip angle $70^{\circ}$, matrix 130x192, FoV $400 \mathrm{~mm}$, slice thickness $6 \mathrm{~mm}, 4 \mathrm{~mm}$ gap). T2P-TrueFisp images were acquired at intervals of at least $3 \mathrm{RR}$ intervals to allow for sufficient magnetisation recovery in between acquisitions.

\subsubsection{WALL MOTION CINE IMAGING}

Routine steady-state free precession sequences were used to acquire long and short axis cine images of the heart (TR $85.8 \mathrm{~ms}$, TE $1.45 \mathrm{~ms}$, flip angle $50^{\circ}$, matrix 173x256, FoV $400 \mathrm{~mm}$, slice thickness $8 \mathrm{~mm}, 2 \mathrm{~mm}$ gap).

\subsubsection{LATE GADOLINIUM ENHANCEMENT IMAGING}

Immediately after the baseline $\mathrm{T} 2$ and $\mathrm{T} 2 *$-weighted scan, participants received an intravenous administration of gadolinium contrast medium $(0.1$ or $0.15 \mathrm{mmol} / \mathrm{kg}$; Gadovist, Bayer Plc, Germany) followed by breath held inversion recovery sequences in long axis and short axis planes to acquire late enhancement images. Gadolinium-enhanced imaging is the current gold standard for infarct detection using MRI. Optimal inversion time (TI) was determined on a slice-by-slice basis using standard late enhancement TI-scout protocols (TR $750 \mathrm{~ms}$, TE $2.61 \mathrm{~ms}$, flip angle $20^{\circ}$, matrix $173 \times 256$, FoV $400 \mathrm{~mm}$, slice thickness $9 \mathrm{~mm}, 1 \mathrm{~mm}$ gap). The inversion recovery late enhancement short axis slices were acquired using similar slice positions as the $\mathrm{T} 2$-oedema and $\mathrm{T} 2 *$-weighted imaging. 


\subsection{STUDY PROTOCOLS (see Figures 2.1a-d)}

Protocols were individually designed for each study.

\subsubsection{HEALTHY VOLUNTEERS}

One pair of pre and 24 hours post USPIO-enhanced MRI scans was sufficient to determine 'normal' tissue uptake of USPIO (Chapter 3).

\subsubsection{MYOCARDIAL INFARCTION}

A variable study protocol was used that was purposely designed for imaging at different time points after acute MI. Over a 3-month period following MI, patients received up to 7 MRI scans and up to 3 infusions of USPIO. Two principal analyses were performed using data from the same participants.

First, to assess the duration of a single dose of ultrasmall superparamagnetic particles of iron oxide, R2* values of participants that first received USPIO within 7 days of myocardial infarction were followed on all subsequent MRI scans prior to receiving any further doses of USPIO (Chapter 4). Second, to assess the duration of myocardial macrophage activity, USPIO uptake was compared using repeated USPIO infusions over the 3-month follow up period. USPIO uptake was calculated by subtracting the baseline $\mathrm{R} 2 *$ value from values obtained at 24 hours following USPIO infusion (Chapter 4). This allowed repeated assessment of myocardial USPIO uptake. Finally, to assess myocardial oedema, T2 values were compared on unenhanced scans, (prior to, and at least 7 days following USPIO administration). 
Following clinical guidance for the administration of ferumoxytol, participants were allowed a maximum of two doses of USPIO in a 1-month period, and a total of three over the 3-month follow up period to prevent iatrogenic iron overload. It was therefore not possible to perform repeated USPIO-enhanced imaging at every time point in the same patient.

\subsubsection{ACUTE MYOCARDITIS AND CARDIAC TRANSPLANTATION}

To assess myocardial macrophage activity in patients with acute myocarditis and cardiac transplantation, USPIO uptake was calculated at baseline, and repeated after 3 months (Chapters 5 and 6 ). 
PARTICLES OF IRON OXIDE AND BLOOD TESTING

Intravenous infusion of USPIO (ferumoxytol, $4 \mathrm{mg} / \mathrm{kg}$ ) (Figure 2.1) was performed immediately following the baseline, and selected subsequent magnetic resonance scans (depending on above protocol) over at least 15 minutes using a concentration of $2-8 \mathrm{mg} / \mathrm{mL}$, diluted in $0.9 \%$ saline or $5 \%$ dextrose. Haemodynamic monitoring was conducted throughout and patients were observed for at least 30 minutes following administration to ensure no hypersensitivity reactions.

Routine blood tests were collected at all visits other than when patients attended for the 24 hour post USPIO MRI scan (as they had been collected the previous day). We measured full blood count, urea and electrolytes, C-reactive protein (CRP) and high sensitivity troponin.

\subsection{IMAGE ANALYSIS}

Several methods of analysis were attempted, but only those retained for data presented in this thesis will be outlined. Using identical methodology to that described throughout this thesis, Alam et al found excellent repeatability for the assessment of R2* with a mean bias of $-0.208 \mathrm{~s}^{-1}$, coefficient of repeatability of $26.96 \mathrm{~s}-1$, and intraclass coefficient of 0.989 [Alam et al 2012]. 


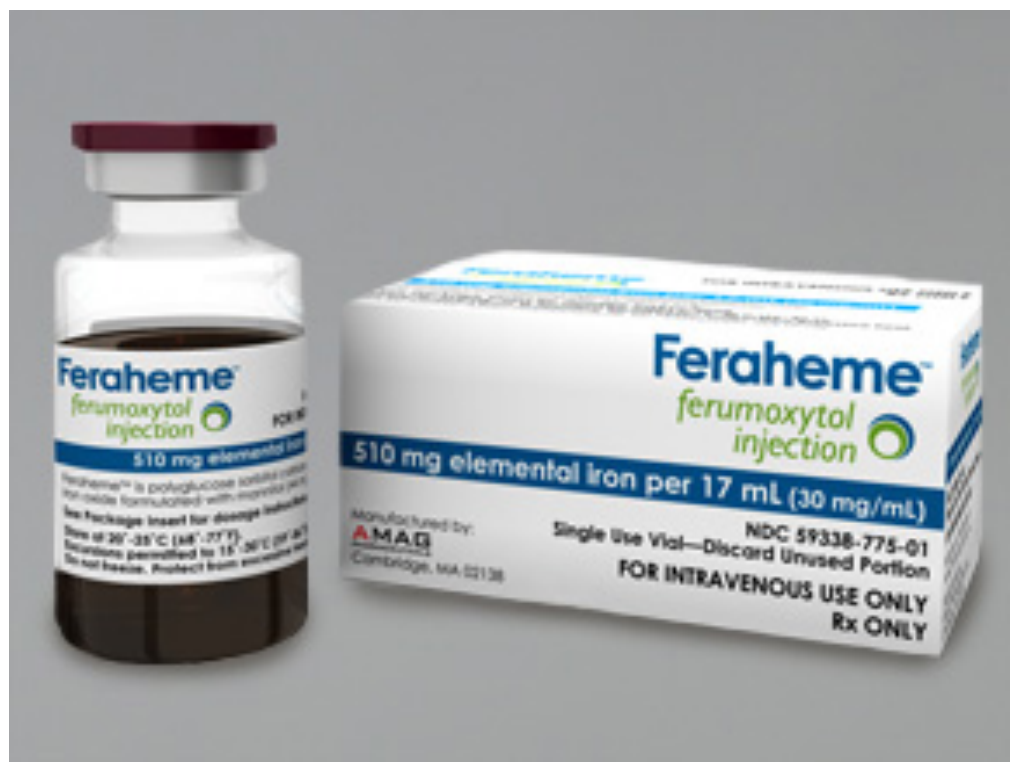

Figure 2.1. Vial of ferumoxytol - $510 \mathrm{mg}$ in $17 \mathrm{~mL}$ (note trade name of Feraheme ${ }^{\mathrm{TM}}$ in the USA; previously licensed as Rienso ${ }^{\circledR}$ in the EU).

\subsubsection{T2 MAP AND T2* ANALYSIS}

All T2 map and T2*-weighted multi-gradient-echo images for each patient were analysed using Circle CVI software (Circle CVI42, Canada). For T2* analysis, an experimentally determined threshold used in previous work [Alam et al 2010] for the coefficient of determination $\left(r^{2}>0.85\right)$ was used to exclude data that did not have an acceptable exponential decay when signal intensity was plotted against echo time. The relaxation rate, $\mathrm{R} 2 *$, is the inverse of the mean $\mathrm{T} 2 *$, and was calculated to assess the uptake of USPIO for each region of interest (ROI), where the higher the value, the greater the USPIO accumulation.

$\mathrm{T} 2$ and $\mathrm{T} 2 *$ values were measured immediately prior to USPIO administration. USPIO-enhanced $\mathrm{T} 2 *$ data was also collected 24 hours following ferumoxytol administration (see Region of Interest Selection below). 


\subsubsection{FUNCTIONAL ANALYSIS}

Ventricular volume and functional analyses were performed using QMass software (Medis Medical Imaging Systems, The Netherlands). Endocardial and epicardial contours were manually drawn on short axis slices through the heart in end-diastole and end-systole from the base of the heart at the level of the mitral valve annulus to the apex (Figure 2.2). Data collected included myocardial mass, end-diastolic and end-systolic volumes (indexed to body surface area (BSA)) and finally ejection fraction.

\subsubsection{LATE GADOLINIUM ENHANCEMENT ANALYSIS AND REGION OF INTEREST} SELECTION

Late gadolinium enhancement analysis was performed using Medis Qmass Delayed Signal Intensity (DSI) analysis wizard (Medis Medical Imaging Systems, The Netherlands). LGE images were aligned with each series of T2 and T2* scans at each visit. After drawing LV endocardial and epicardial contours, we then selected and verified areas of healthy and hyperintense (infarct) myocardium, and verified the 

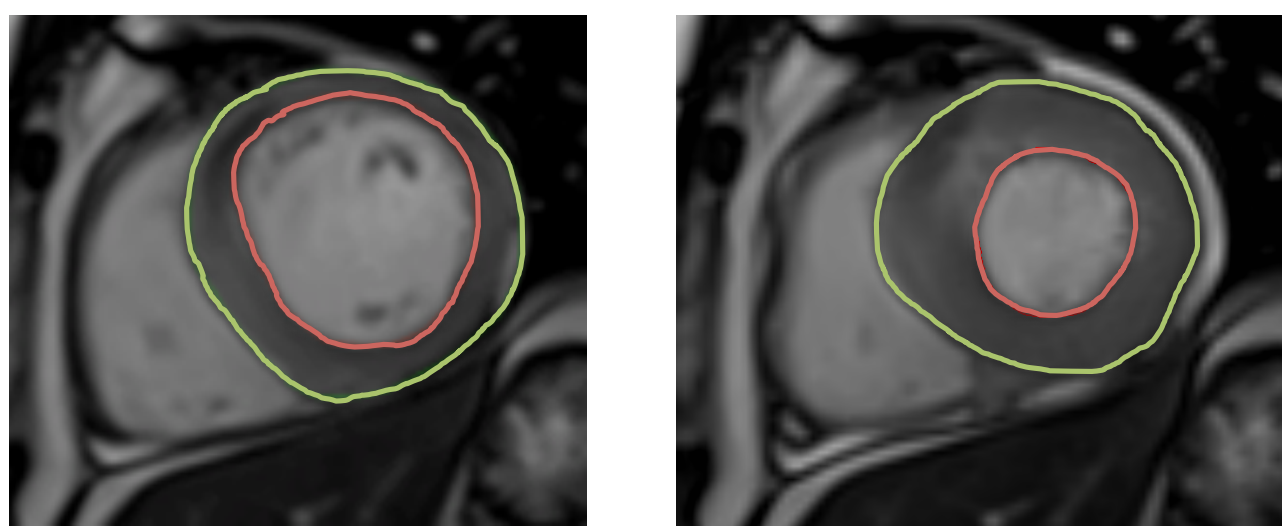

Figure 2.2. Epicardial (green) and endocardial (red) contours for a single short axis slice in enddiastole (left) and end-systole (right) to calculate myocardial mass, end-diastolic and end-systolic volumes, and ejection fraction. 
DSI threshold and segmentation. To delineate areas of LGE, we used two standard deviations above normal remote myocardium, with further adjustments with manual correction if required (Figure 2.3). This allowed myocardial ROIs to be selected on the LGE images and applied to coaligned T2* maps. Regions of interest were also drawn in the heart using standard cardiac segmentation [Cerqueira et al 2002], and pan-myocardial values averaged using segments 1-16 (Figures 2.4 and 2.5).

For patients with myocardial infarction, regions were categorised into infarct zone, peri-infarct zone, and remote myocardium (defined by late gadolinium enhancement, $1 / 2$ segment immediately adjacent to late gadolinium enhancement, and opposite the infarct zone on short axis slice and at least 1 full myocardial segment clear of LGE respectively, Figure 2.6). Where there was not clear delineation between infarct and peri-infarct tissue, the infarct and peri-infarct regions did not meet. This was to ensure the infarct region was purely within infarcted tissue, and the peri-infarct region outwith infarcted tissue.

To focus on inflamed myocardium in patients with acute myocarditis, regions with contiguous LGE $>1 \mathrm{~cm}^{2}$ on a single short axis slice were retained and visually coaligned with corresponding $\mathrm{T} 2$ and $\mathrm{T} 2 *$ images. These corresponding coaligned regions were then averaged to form $\mathrm{LGE}+\mathrm{T} 2$ and $\mathrm{T} 2 *$ regions.

Other selected regions included liver, spleen, bone marrow, kidney, blood pool, aortic wall and skeletal muscle. 


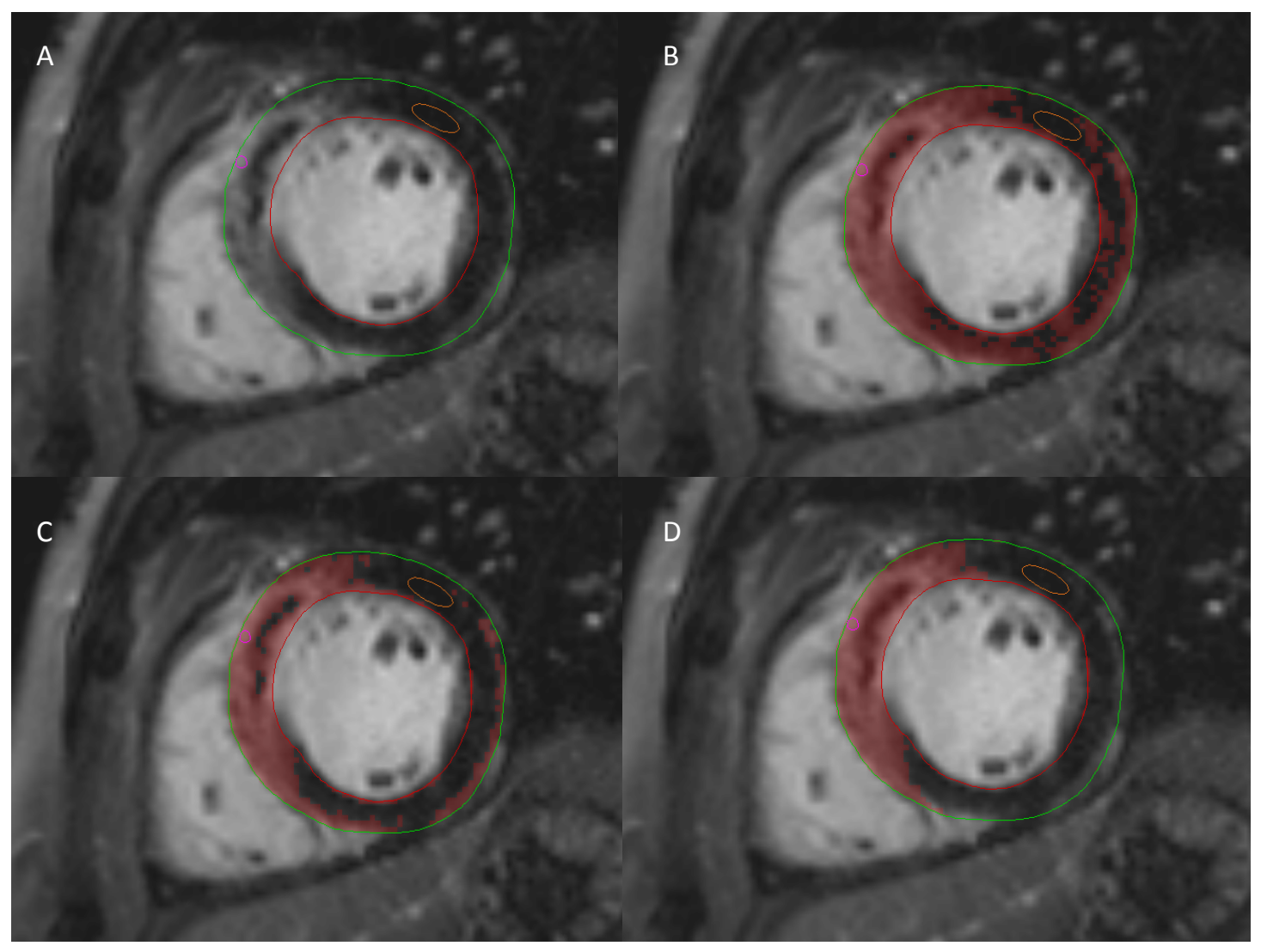

Figure 2.3. LGE selection using Medis Qmass Delayed Signal Intensity (DSI) wizard. After drawing LV endocardial and epicardial contours, we then selected and verified areas of healthy and hyperintense (infarct) myocardium (A), and verified the DSI threshold and segmentation (B). To delineate areas of LGE, we used two standard deviations above normal remote myocardium, with further adjustments with manual correction if required $(\mathbf{C}, \mathbf{D})$. In this example of a large anteroseptal myocardial infarction, reducing the threshold (from B to C), subsequently excluded areas of microvascular obstruction (black core within white infarct area) which then needed to be manually included (C to D). LGE - late gadolinium enhancement; LV - left ventricular. 


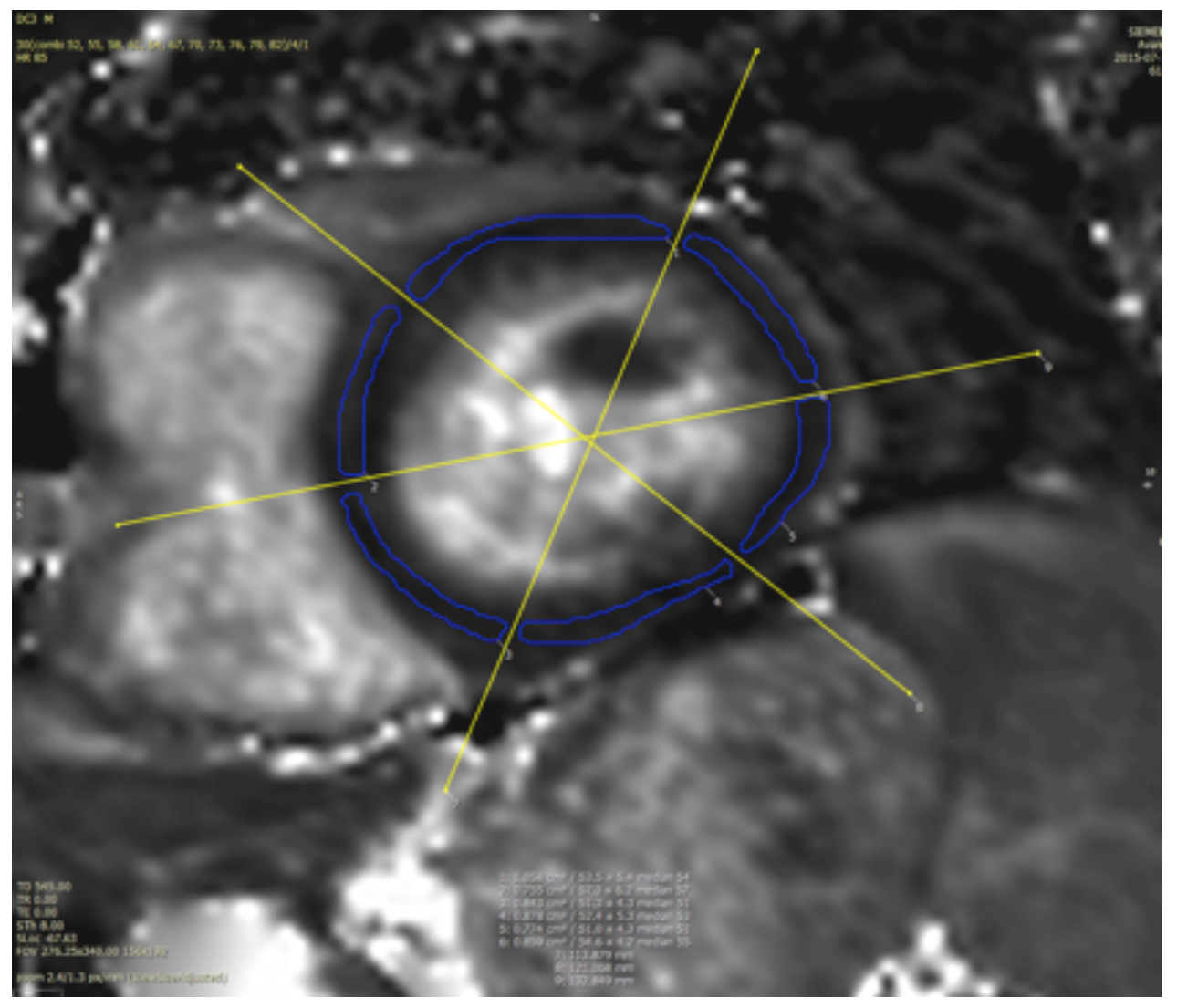

Figure 2.4. Basal short axis slice showing $T 2$ map in greyscale. Segments 1-6 are manually drawn with care taken to ensure sampling of the myocardium only. A mean T2 value (ms) for each segment is provided at the bottom of the image. 


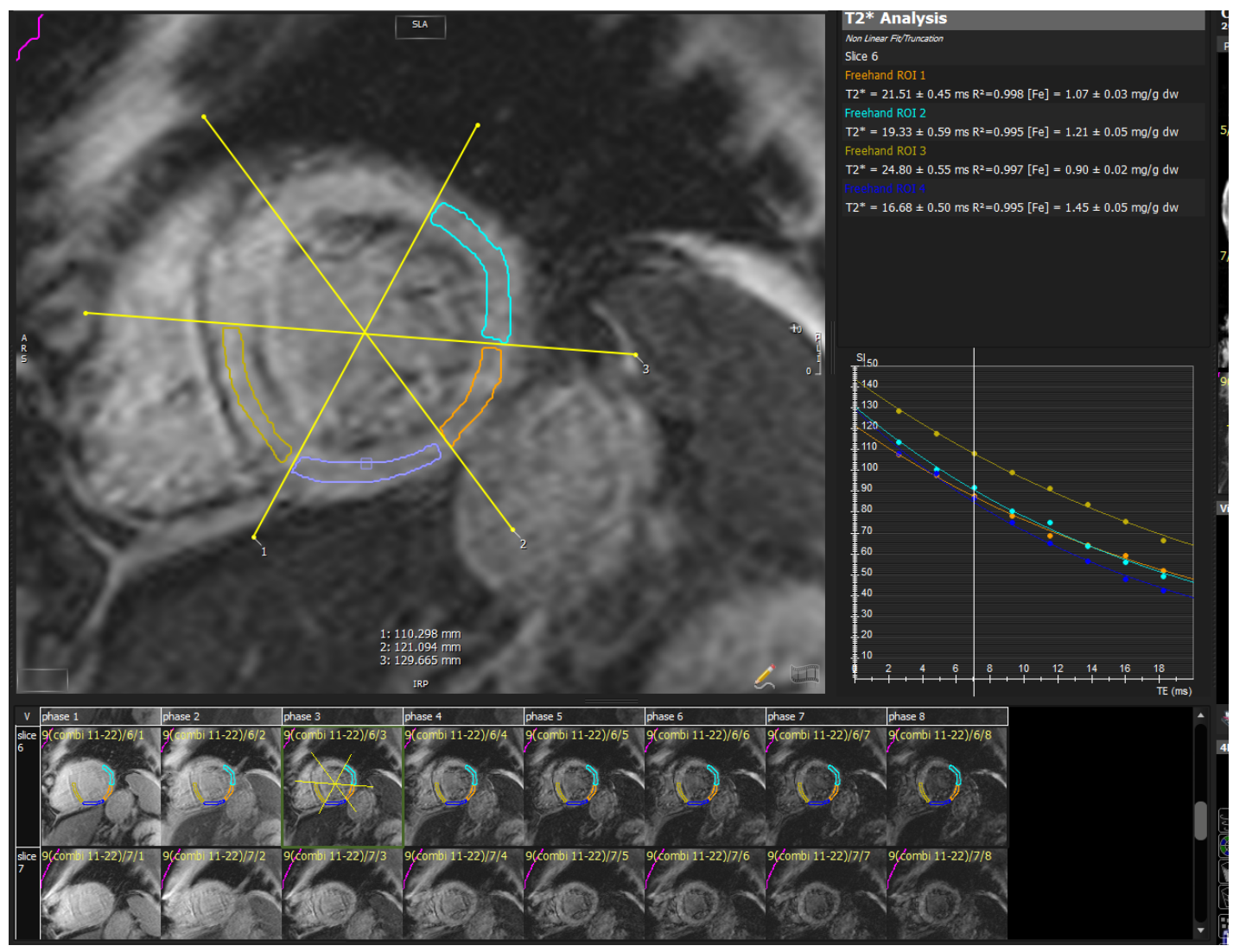

Figure 2.5. Short axis mid ventricular slice of a single T2* echo. Segments 9 (yellow), 10 (purple), 11 (orange) and 12 (turquoise) are selected and the $\mathrm{T} 2 *$ decay curve for each region is displayed on the graph (right). Here, all segments have an $r^{2}$ close to 1 indicating excellent curve fitting. Care is taken to avoid sampling the epicardium and endocardium and regions of artefact. All 8 individual echos for each slice are available for viewing at the bottom of the image to ensure no artefact is included. $\mathrm{r}^{2}$ - coefficient of determination. 


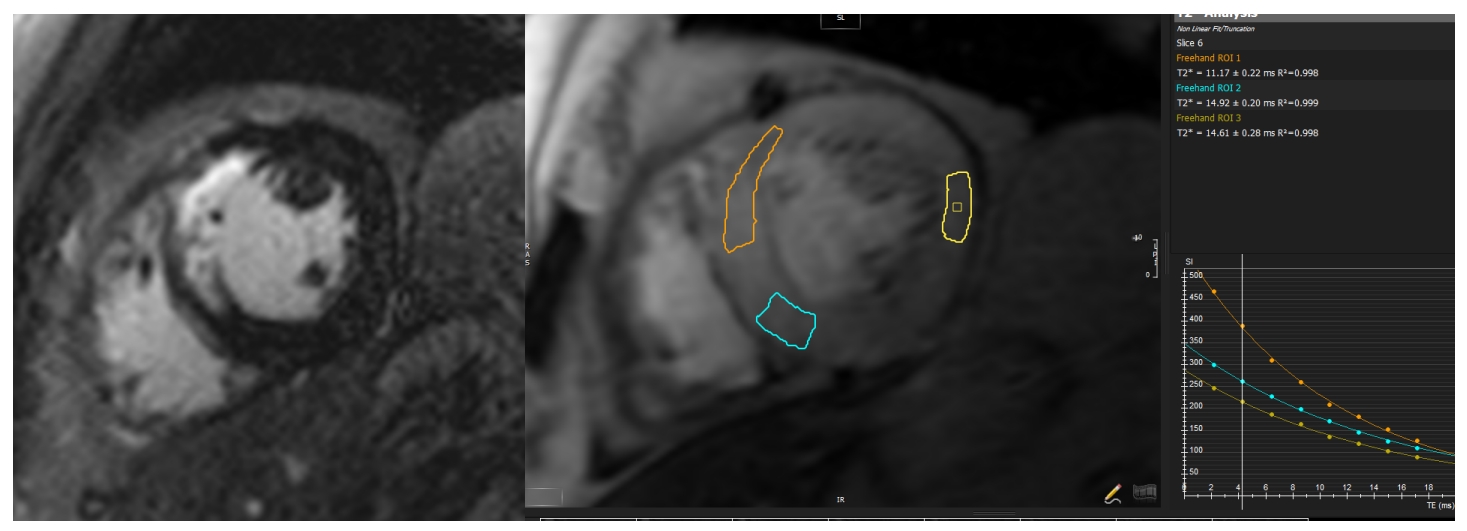

Figure 2.6. Region of interest selection in myocardial infarction. Regions of interest including infarct (orange), peri-infarct (green) and remote (yellow) are drawn with reference to the LGE image. Here all regions on this post USPIO short axis slice display good $\mathrm{T} 2 *$ decay curve fitting $\left(\mathrm{r}^{2}\right.$ close to 1). The infarct zone displays the lowest $\mathrm{T} 2 *$ value (highest $\mathrm{R} 2 *$ ). As the boundary between infarct and peri-infarct tissue was not clear, the infarct region was drawn from purely infarcted tissue, and the peri-infarct region outwith the region of infarction. LGE - late gadolinium enhancement; USPIO - ultrasmall superparamagnetic particles of iron oxide. 


\subsection{HISTOLOGICAL ANALYSIS}

Myocardial tissue samples were obtained from patients undergoing coronary artery bypass graft $(\mathrm{CABG})$ surgery for treatment of their coronary disease within 2 weeks of their index myocardial infarction. A further biopsy was obtained from a patient with cardiac transplant having routine myocardial surveillance biopsies. USPIO was administered 24 hours prior to the biopsy, and a trucut myocardial biopsy sample was taken from the myocardium (infarct area for MI patients) during the procedure. The biopsy sample was fixed in formalin, embedded in paraffin, sectioned, and stained to look at the architecture (haematoxylin and eosin), and the accumulation and distribution of USPIO (Prussian Blue) and macrophages (CD68). Patients that underwent $\mathrm{CABG}$ surgery were excluded from further MRI follow up.

\subsection{STATISTICAL ANALYSIS}

All statistical analysis was performed with GraphPad Prism, version 6 (GraphPad Software, San Diego, CA, USA). To assess duration of USPIO enhancement in tissues following single administration in the first week after myocardial infarction, R2* values from subsequent MRI scans (prior to any further USPIO dosing) were compared using one-way analysis of variance (ANOVA) with Tukey's post hoc test for multiple comparisons. To assess variation and duration of myocardial inflammation after MI, USPIO uptake (R2* increase from pre to 24 hours post USPIO) was compared using one-way ANOVA with Tukey's post hoc test for

multiple comparisons (repeated measures where appropriate). As a further method to 
assess USPIO accumulation within the heart, R2* values 24 hours after administration (without baseline R2* subtraction) was compared in the same way. Finally, to assess variation and duration of myocardial oedema after MI, T2 values were compared again using one-way ANOVA with Tukey's post hoc test for multiple comparisons (repeated measures where appropriate).

To compare USPIO uptake and myocardial oedema between groups (volunteers with either patients with acute myocarditis or cardiac transplantation), R2* and T2 values were compared using unpaired $t$-test. To compare results at 3 months with baseline within patient groups, a paired $t$-test was used. Statistical significance was defined as two-sided $\mathrm{p}<0.05$ for all statistical tests mentioned above.

\subsection{SAMPLE SIZE CALCULATIONS}

Previous work [Alam et al 2012] has shown the change in R2* due to USPIO in 'healthy' myocardium (remote from the site of myocardial infarction) to be $40+/-11$ $\mathrm{s}^{-1}$ at 24 hours following USPIO. Assuming the change due to USPIO is similar in truly healthy myocardium, a sample size of 10 in each group is needed to detect an effect size of $20 \mathrm{~s}^{-1}$ due to USPIO, (ie an increase of $50 \%$ ) with $80 \%$ power and significance level of 0.05 . 
CHAPTER 3

FERUMOXYTOL-ENHANCED MAGNETIC RESONANCE IMAGING;

METHODOLOGY AND NORMAL VALUES

AT 1.5 TESLA AND 3 TESLA 


\subsection{SUMMARY}

Background Ultrasmall superparamagnetic particles of iron oxide-enhanced magnetic resonance imaging can detect tissue-resident macrophage activity and identify cellular inflammation. Clinical studies using this technique are now emerging. I aimed to report a range of normal R2* values at 1.5 tesla and 3 tesla in the myocardium and other tissues following ferumoxytol administration, outline the methodology used and suggest solutions to commonly encountered analysis problems.

Methods Twenty volunteers were recruited: 10 imaged each at $1.5 \mathrm{~T}$ and $3 \mathrm{~T}$. T2* and late gadolinium-enhanced MRI was conducted at baseline with further T2* imaging conducted approximately 24 hours after USPIO infusion (ferumoxytol, $4 \mathrm{mg} / \mathrm{kg}$ ). Regions of interest were selected in the myocardium and compared to other tissues.

Results Following administration, USPIO were detected by changes in R2* from baseline $(1 / \mathrm{T} 2 *)$ at 24 hours in myocardium, skeletal muscle, kidney, liver, spleen and blood at $1.5 \mathrm{~T}$, and myocardium, kidney, liver, spleen, blood and bone at $3 \mathrm{~T}$ ( $p<0.05$ for all). Myocardial changes in R2* due to USPIO were $26.5 \pm 7.3 \mathrm{~s}^{-1}$ at $1.5 \mathrm{~T}$, and $37.2 \pm 9.6 \mathrm{~s}^{-1}$ at $3 \mathrm{~T}(\mathrm{p}<0.0001$ for both). Tissues showing greatest ferumoxytol enhancement were in the reticuloendothelial system: the liver, spleen and bone marrow $\left(216.3 \pm 32.6 \mathrm{~s}^{-1}, 336.3 \pm 60.3 \mathrm{~s}^{-1}, 69.9 \pm 79.9 \mathrm{~s}^{-1} ; \mathrm{p}<0.0001, \mathrm{p}<0.0001, \mathrm{p}=\mathrm{ns}\right.$ respectively at $1.5 \mathrm{~T}$, and $275.6 \pm 69.9 \mathrm{~s}^{-1}, 463.9 \pm 136.7 \mathrm{~s}^{-1}, 417.9 \pm 370.3 \mathrm{~s}^{-1} ; \mathrm{p}<0.0001$, $\mathrm{p}<0.0001, \mathrm{p}<0.01$ respectively at $3 \mathrm{~T})$. 
Conclusion Ferumoxytol-enhanced MR imaging is feasible at both $1.5 \mathrm{~T}$ and $3 \mathrm{~T}$. Careful data selection and dose administration, along with refinements to echo time acquisition, post processing and analysis techniques are essential to ensure reliable and robust quantification of tissue enhancement.

\subsection{INTRODUCTION}

Iron oxide nanoparticles are a class of magnetic resonance imaging contrast agents that are generating interest as a method of detecting tissue inflammation. Historically, these nanoparticles were initially used for gastrointestinal, reticuloendothelial system and lymph node imaging [Saini et al 1987; Hahn et al 1990; Rogers et al 1994], and subsequently in hepatic and cardiac imaging [Canet et al 1993; Ros et al 1995; Kroft et al 1998; Taylor et al 1999]. Recently however, it is in their use as an MRI contrast agent for detecting tissue-resident macrophages that clinical applications are now emerging [Trivedi et al 2004; Tang et al 2006; Trivedi et al 2006; Tang et al 2009; Richards et al 2011; Alam et al 2012; Yilmaz et al 2013; McBride et al 2015].

T2* MR imaging has been successfully used for over a decade in diagnosing and grading severity of iron accumulation in transfusion-dependent thalassaemia major, and has been instrumental in guiding therapy that improves prognosis, and allows serial disease monitoring [Anderson et al 2001; Anderson et al 2004]. T2* MRI in the assessment of iron accumulation is easily quantifiable, well validated, highly reproducible, clinically robust, and is achievable in a single breath-hold 
[Westwood et al 2003a; Westwood et al 2003b; Carpenter et al 2009; Kirk et al 2010; Carpenter et al 2011].

Ultrasmall superparamagnetic particles of iron oxide consist of an iron oxide core surrounded by a carbohydrate or polymer coating. These particles can extravasate through damaged capillaries, where they are engulfed and concentrated by tissueresident macrophages [Ruehm et al 2001]. Gradient echo T2*-weighted sequences are highly sensitive to magnetic field inhomogeneities such as susceptibility artefacts due to the presence of iron, including USPIO. Accumulation of USPIO in macrophages can be quantified and visualised using T2*W MR imaging [Alam et al 2012; Yilmaz et al 2013] and calculation of, and observing the reduction in, T2* relaxation time due to the presence of iron. Thus USPIO-enhanced MRI can detect tissue-resident macrophage activity and identify localised cellular inflammation within tissues.

In this present study I aimed to observe and quantify the distribution of ferumoxytol enhancement following intravenous administration at $1.5 \mathrm{~T}$ and $3 \mathrm{~T}$ MRI and establish a range of normal healthy myocardial and other tissue values. I also aimed to develop our methodology and describe commonly encountered problems in $\mathrm{T} 2 *$ image analysis of USPIO. 


\subsection{METHODS}

This was an open label observational multicentre cohort study using human volunteers recruited as part of a larger trial, recruiting patients with cardiac inflammation. The study was performed in accordance with the Declaration of Helsinki, the approval of the Scotland A Research Ethics Committee, and the written informed consent of all participants.

\subsubsection{SUBJECTS}

Participants were aged over 18 years of age. Exclusion criteria were contraindication to MR imaging or ferumoxytol infusion, any systemic inflammatory comorbidity (eg rheumatoid arthritis), renal failure (estimated glomerular filtration rate $<30 \mathrm{~mL} / \mathrm{min}$ ), pregnancy, breastfeeding and women of childbearing age not ensuring reliable contraception.

\subsubsection{MAgNeTIC RESONANCE IMAGING}

MRI was performed using $3 \mathrm{~T}$ and $1.5 \mathrm{~T}$ scanners (Magnetom Verio and Avanto respectively), with dedicated cardiac array coils. All images were acquired using electrocardiogram-gated breath-hold imaging. Routine steady state free precession sequences were used to acquire long axis and short axis images of the heart. Standard cardiac slice widths (6 mm width with $4 \mathrm{~mm}$ gap) and 8 echo times (2.1-17.1 $\mathrm{ms}$ range) with matrix size of $256 \times 115$ were acquired in order to generate $\mathrm{T} 2 *$ maps. The in-plane resolution differed as required for larger or smaller subjects; generally, a field of view of $400 \times 300 \mathrm{~mm}$ was used with an in-plane resolution of $2.6 \times 1.6 \mathrm{~mm}$. 
T2* relaxation maps were generated before and approximately 24 hours after administration of USPIO.

Immediately after the baseline T2* and SSFP cine imaging, breath held inversion enhancement images were acquired following an intravenous administration of gadolinium contrast medium $(0.1$ and $0.15 \mathrm{mmol} / \mathrm{kg}$ at $3 \mathrm{~T}$ and $1.5 \mathrm{~T}$ respectively). Optimal inversion time was determined on a slice-by-slice basis using standard late enhancement TI-scout protocols. The inversion recovery late enhancement short axis slices were acquired using similar slice positions to the myocardial $\mathrm{T} 2 *$ imaging. The $\mathrm{T} 2 *$ acquisitions also included imaging of the liver, spleen and spine to allow quantification of USPIO accumulation within organs of the reticuloendothelial system.

\subsubsection{USPIO}

Intravenous infusion of USPIO (ferumoxytol, $4 \mathrm{mg} / \mathrm{kg}$ ) was performed immediately following the baseline magnetic resonance scan over at least 15 minutes using a concentration of $2-8 \mathrm{mg} / \mathrm{mL}$, diluted in $0.9 \%$ saline or $5 \%$ dextrose. Haemodynamic monitoring was conducted throughout.

\subsubsection{STUDY PROTOCOL}

Volunteers received two MRI scans approximately 24 hours apart (Figure 3.1).

\subsubsection{IMAGE ANALYSIS}

All T2*-weighted multi-gradient-echo images for each patient were analysed using 
Circle CVI software. Regions of interest were drawn in the heart using standard cardiac segmentation [Cerqueira et al 2002], and pan-myocardial values averaged using segments 1-16. Further regions of interest were drawn in skeletal muscle, kidney, liver, spleen, blood pool (from the LV cavity) and bone marrow.

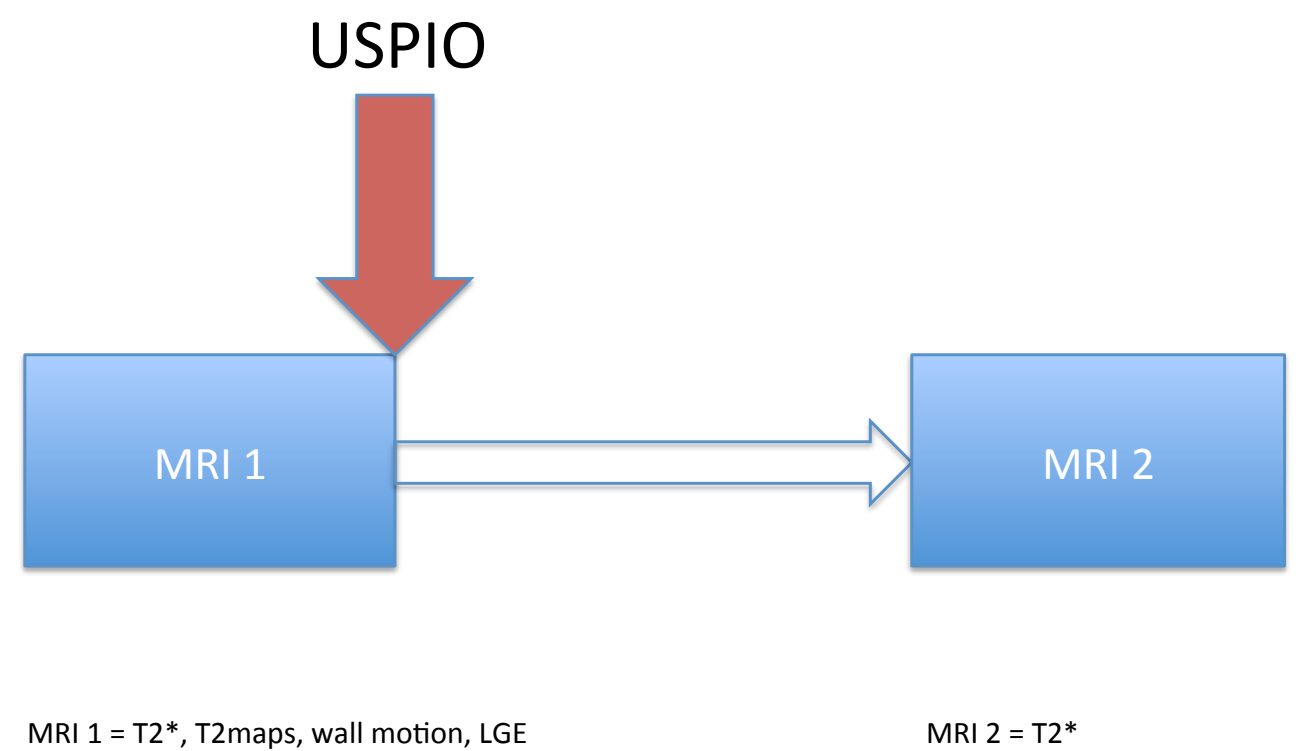

Figure 3.1. MRI Protocol.

USPIO - ultrasmall superparamagnetic particles of iron oxide; MRI - magnetic resonance imaging; LGE - late gadolinium enhancement. 
An experimentally determined threshold used in previous work [Alam et al 2012] for the coefficient of determination $\left(r^{2}>0.85\right)$ was used to exclude data that did not have an acceptable exponential decay when signal intensity was plotted against echo time. The inverse of the mean $\mathrm{T} 2 *(\mathrm{R} 2 *)$ for each ROI was then calculated to assess the uptake of USPIO, where the higher the value, the greater the USPIO accumulation.

Late gadolinium enhancement, ventricular volume and functional analyses were performed using Circle CVI software. T2* data were collected immediately prior to USPIO administration. USPIO-enhanced $\mathrm{T} 2 *$ data were collected $24-25$ hours following ferumoxytol administration.

\subsubsection{STATISTICAL ANALYSIS}

All statistical analysis was performed with GraphPad Prism, version 6. To assess uptake of USPIO in tissues following single administration, R2* increase from pre to 24 hours following USPIO were compared using repeated measures one-way ANOVA. Univariate and multivariable linear regression models were constructed to examine the association of clinically relevant variables with myocardial R2* uptake. As myocardial R2* uptake was highly skewed, this was log transformed. Statistical significance was defined as two-sided $\mathrm{p}<0.05$. 


\subsection{RESULTS}

Twenty volunteer patients were recruited in total (10 at $1.5 \mathrm{~T}, 10$ at $3 \mathrm{~T})$. Forty MRI scans and 20 infusions of ferumoxytol were completed over the course of the study. Data from one participant at $1.5 \mathrm{~T}$ has been removed due to the presence of LGE, (which was included in the MRI protocol so that we could exclude volunteers with any detectable cardiac MRI abnormalities according to standard MRI protocols). All other volunteers that were included had structurally normal hearts. One participant was prescribed antihypertensive medication but had a normal CMR study and was normotensive so the data was retained for analysis. Administration of ferumoxytol was well tolerated with no adverse reactions reported during or immediately after administration in any of the participants.

Participants were predominantly middle-aged, with greater numbers of women in both groups (Table 3.1). There were no differences between $1.5 \mathrm{~T}$ and $3 \mathrm{~T}$ groups in body mass index (BMI) or ejection fraction at baseline.

A summary of results is shown in Table 3.2. At baseline, pan-myocardial R2* values were greater at $3 \mathrm{~T}$ than $1.5 \mathrm{~T}\left(46.9 \pm 4.1\right.$ versus $33.5 \pm 5.4 \mathrm{~s}^{-1}$, Figure $\left.3.2 ; \mathrm{p}<0.01\right)$ as expected. Baseline R2* values were also greater at $3 \mathrm{~T}$ in bone $(\mathrm{p}<0.0001)$ but no baseline differences were seen between magnetic field strength in all other tissues (Figure 3.3; $\mathrm{p}>0.05$ for all). USPIO increased pan-myocardial R2* values at 24 hours in both $1.5 \mathrm{~T}$ and $3 \mathrm{~T}$ scanners $(\mathrm{p}<0.0001$ for both). Post USPIO panmyocardial R2* values were again greater at $3 \mathrm{~T}$ than $1.5 \mathrm{~T}$, as expected 
(84.2 \pm 12.4 versus $\left.60.5 \pm 7.2 \mathrm{~s}^{-1} ; \mathrm{p}<0.0001\right)$. Pan-myocardial change in R2* between baseline and 24 hours post USPIO at $1.5 \mathrm{~T}$ was $26.5 \pm 7.3 \mathrm{~s}^{-1}$ and at $3 \mathrm{~T}$ was $37.2 \pm 9.6 \mathrm{~s}^{-1} ;(\mathrm{p}<0.0001$ for both). Detectable increases in $\mathrm{R} 2 *$ were also observed at 24 hours post USPIO in skeletal muscle, kidney, liver, spleen and blood at $1.5 \mathrm{~T}$, and kidney, liver, spleen, blood and bone at $3 \mathrm{~T}$ (Figure 3.3; $\mathrm{p}<0.05$ for all).

BMI correlated with the pan-myocardial R2* changes due to USPIO contrast (Figure 4.4; $\mathrm{r}=0.72, \mathrm{p}<0.001$ ). With univariate analysis, age, weight and BMI were all associated with $\mathrm{R} 2$ value $(\mathrm{P}<0.05$ for all, Table 3.3$)$. However, in a multivariable adjusted model, only BMI was independently associated with $\mathrm{R} 2$ value $(\mathrm{P}=0.026$, Table 3.3).

TABLE 3.1 Participant Characteristics

\begin{tabular}{lll}
\hline & $\mathbf{1 . 5}$ T & $\mathbf{3 ~ T}$ \\
\hline Number & 9 & 10 \\
Male/Female & $3: 6$ & $4: 6$ \\
Age & $52(45.5-61)$. & $50(45.25-53)$ \\
BMI $\left(\mathrm{kg} / \mathrm{m}^{2}\right)$ & $22.9(20.1-26.9)$ & $25.9(22.5-29.4)$ \\
Ejection fraction (\%) & $63.6 \pm 4.9$ & $61.1 \pm 4.1$ \\
& \\
& \\
N (\%), mean \pm SD, or median (interquartile range). \\
T - tesla; BMI - body mass index; SD - standard deviation.
\end{tabular}




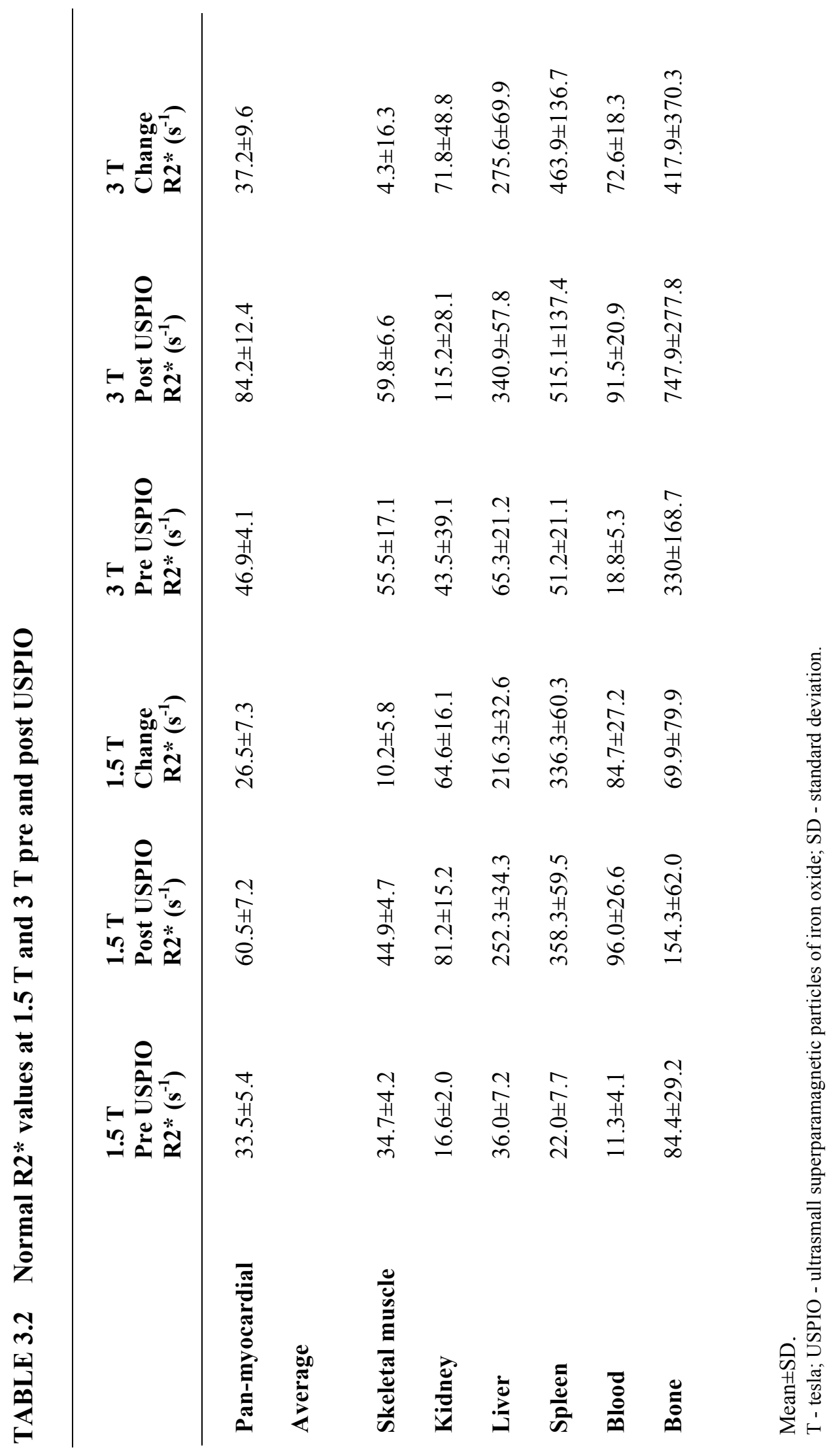




\section{Pan-myocardial average}

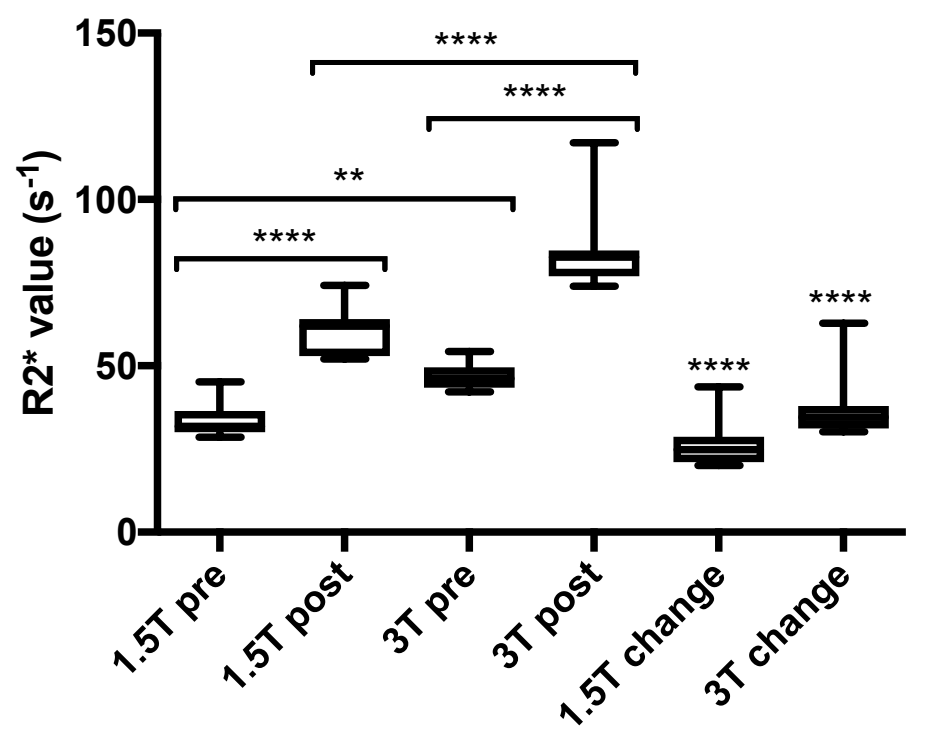

Figure 3.2. Myocardial R2* pre and post USPIO administration at 1.5 tesla and 3 tesla. Following administration, USPIO was detected by an increase in R2* at 24 hours in the myocardium at both $1.5 \mathrm{~T}$ and $3 \mathrm{~T}(* * * *=\mathrm{p}<0.0001, * *=\mathrm{p}<0.01)$. USPIO - ultrasmall superparamagnetic particles of iron oxide; $h$ - hours. 

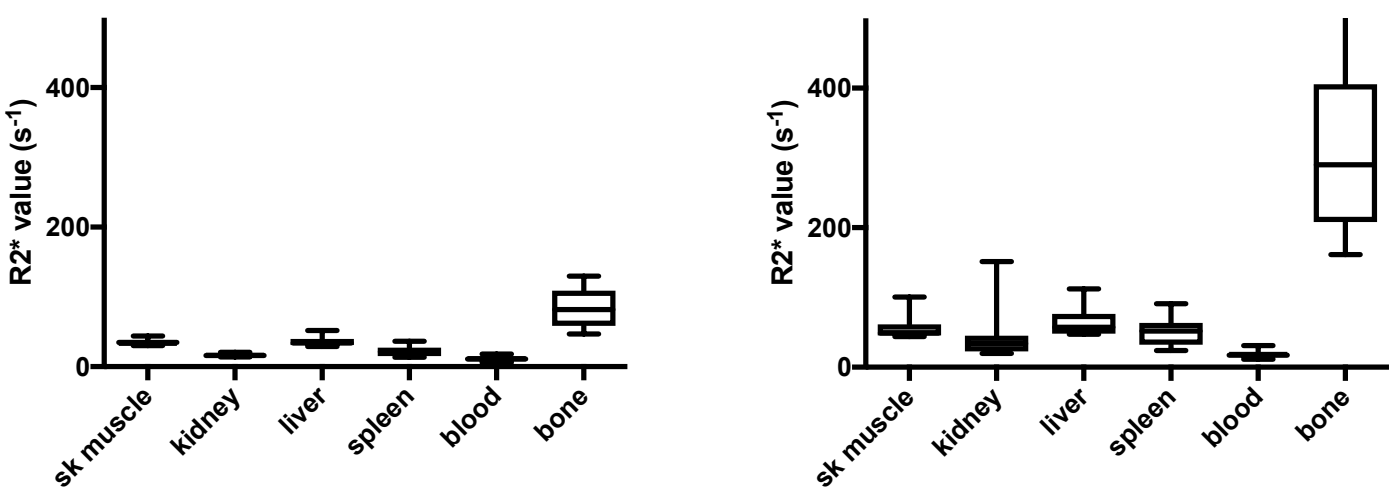

1.5T Post USPIO R2*

3T Post USPIO R2*
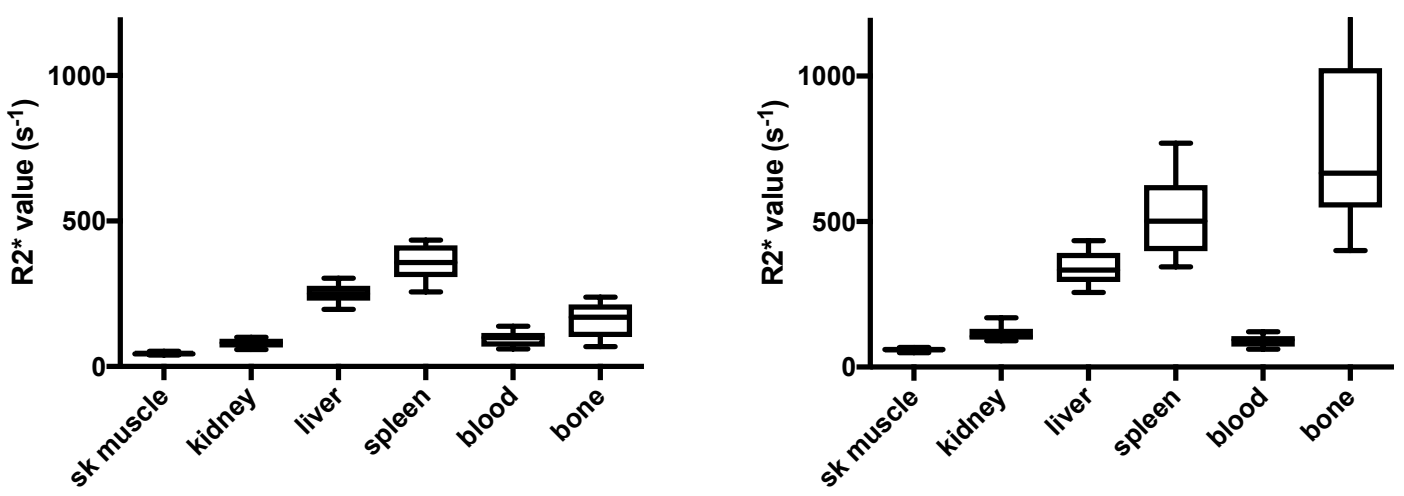

1.5T Change R2*
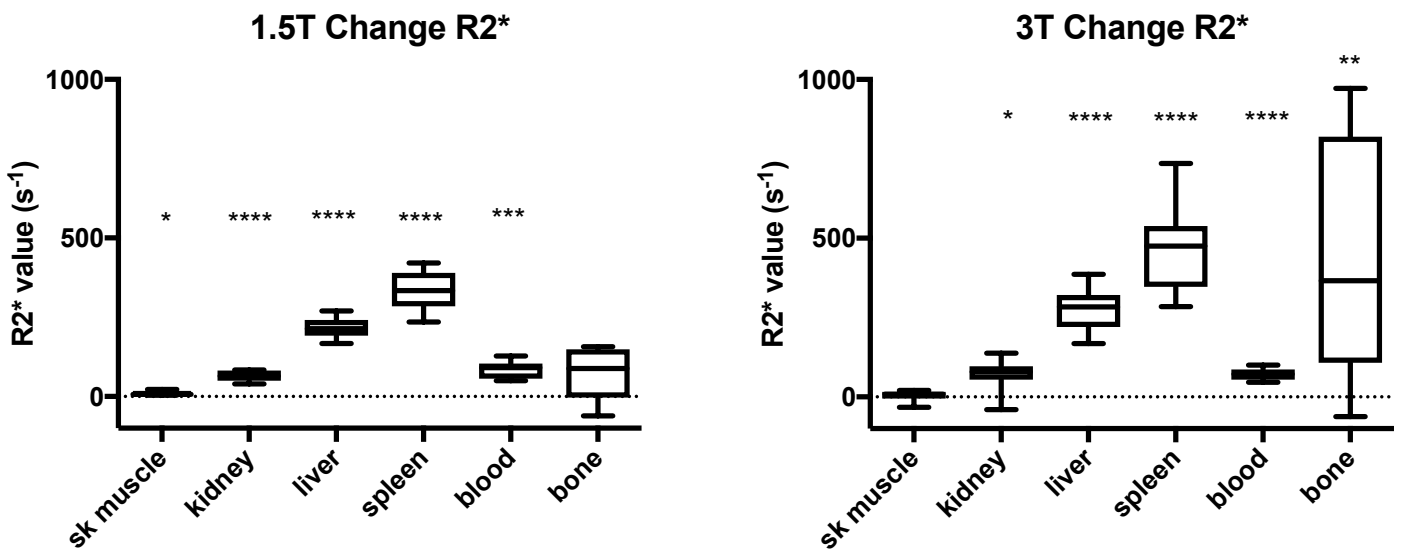

Figure 3.3. Tissue R2* pre and post USPIO administration at 1.5 tesla and 3 tesla. Following administration, USPIO was detected by an increase in R2*, 24 hours after administration in skeletal muscle, kidney, liver, spleen and blood at $1.5 \mathrm{~T}$, and kidney, liver, spleen, blood and bone at $3 \mathrm{~T}$ $(* * * *=\mathrm{p}<0.0001, * * *=\mathrm{p}<0.001, * *=\mathrm{p}<0.01, *=\mathrm{p}<0.05)$. USPIO - ultrasmall superparamagnetic particles of iron oxide; $\mathrm{h}$ - hours. 


\section{BMI vs Pan-myocardial R2* change}

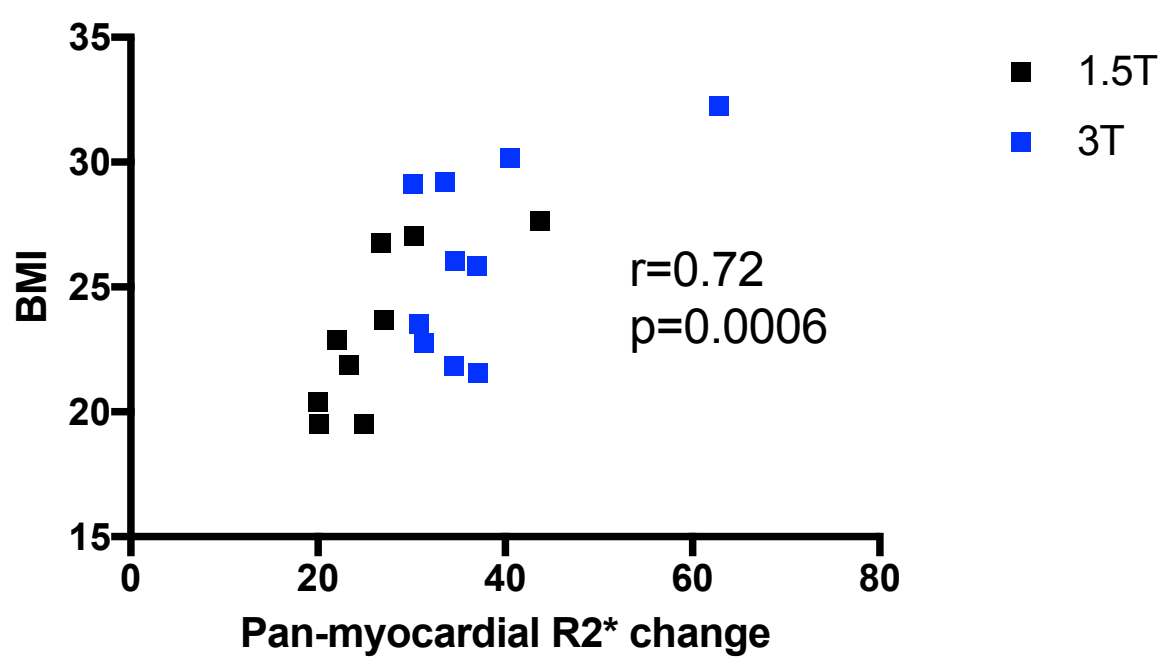

Figure 3.4. Body mass index versus pan-myocardial R2* change at 1.5 tesla and 3 tesla. Body mass index correlates with pan-myocardial R2* change pre and post USPIO. BMI - body mass index; USPIO - ultrasmall superparamagnetic particles of iron oxide. 
TABLE 3.3 ASSOCIATION OF VARIABLES WITH PAN-MYOCARDIAL R2* UPTAKE

\author{
Univariate \\ Relative change in $\mathrm{P}$ value \\ R2* value \\ $(95 \% \mathrm{CI})$
}

\begin{tabular}{|c|c|c|c|c|}
\hline $\begin{array}{l}\text { Age, per } 1 \text { year } \\
\text { increase }\end{array}$ & $\begin{array}{l}0.98 \\
(0.97 \text { to } 0.99)\end{array}$ & 0.001 & $\begin{array}{l}0.99 \\
(0.98 \text { to } 1.01)\end{array}$ & 0.415 \\
\hline Sex (Female) & $\begin{array}{l}0.94 \\
(0.70 \text { to } 1.28)\end{array}$ & 0.688 & $\begin{array}{l}1.08 \\
(0.76 \text { to } 1.55)\end{array}$ & 0.634 \\
\hline $\begin{array}{l}\text { Height, per cm } \\
\text { increase }\end{array}$ & $\begin{array}{l}1.00 \\
(0.99 \text { to } 1.02)\end{array}$ & 0.630 & $\begin{array}{l}1.08 \\
\text { (1.00 to } 1.17)\end{array}$ & 0.050 \\
\hline $\begin{array}{l}\text { Weight, per kg } \\
\text { increase }\end{array}$ & $\begin{array}{l}1.01 \\
(1.00 \text { to } 1.02)\end{array}$ & 0.020 & $\begin{array}{l}0.91 \\
(0.83 \text { to } 1.00)\end{array}$ & 0.055 \\
\hline $\begin{array}{l}\text { BMI, per unit } \\
\text { increase }\end{array}$ & $\begin{array}{l}1.06 \\
(1.03 \text { to } 1.09)\end{array}$ & $<0.001$ & $\begin{array}{l}1.36 \\
\text { (1.04 to } 1.76)\end{array}$ & 0.026 \\
\hline
\end{tabular}

Univariate and multivariable linear regression analysis to examine association of variables with R2* value. R2* values were log-transformed as these were highly skewed. Multivariable linear regression model: adjusted $\mathrm{R}^{2^{*}} 0.66 ; \mathrm{P}=0.001$.
Multivariable

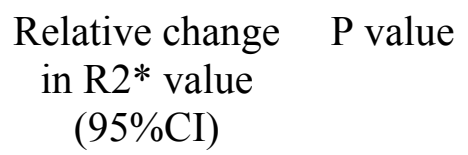

. 


\subsection{DISCUSSION}

For the first time, I report a range of normal T2* values in the healthy human heart and other tissues 24 hours after ferumoxytol administration at $1.5 \mathrm{~T}$ and $3 \mathrm{~T}$. I will also describe problems, solutions and guidance in ferumoxytol-enhanced $\mathrm{T} 2 *$ image analysis.

Following administration, USPIO is detectable by $\mathrm{T} 2 *$ imaging in the myocardium and other tissues at both $1.5 \mathrm{~T}$ and $3 \mathrm{~T}$. Tissues with small increases in R2* (less than the blood pool) are likely to represent detection of USPIO within the intravascular space and include skeletal muscle (at $1.5 \mathrm{~T}$ only), myocardium and kidney. In contrast, R2* changes that are greater than the blood pool must be due to accumulation of USPIO, either through iron storage, uptake by macrophages or other phagocytes, or sequestered within tissue interstitium. In the absence of tissue biopsies, I cannot be certain, but as the most pronounced R2* changes were seen in the spleen, liver and bone marrow (organs of the reticuloendothelial system), it would appear likely that USPIO is incorporated quickly into tissue-resident phagocytes and macrophages.

Detection of USPIO enhancement in skeletal muscle at $1.5 \mathrm{~T}$ but not $3 \mathrm{~T}$ is due to generally noisier data seen across all tissues at $3 \mathrm{~T}$. Due to wider data confidence intervals, a larger sample size would be required to detected the same mean change in $\mathrm{R} 2 *$. The variation in data at $3 \mathrm{~T}$ is partly artefact in the images, but also because of the lower values at $3 \mathrm{~T}$ (USPIO has a faster T2* decay time at $3 \mathrm{~T}$ ). With the same 
sampling echo times, there are fewer data points to construct the decay curve at $3 \mathrm{~T}$ than $1.5 \mathrm{~T}$ so our error in estimation also increases.

I chose 24 hours post USPIO to re-image participants as myocardial signal attenuation at 24 hours has shown to be optimal in the myocardium compared to later time points [Alam et al 2012; Yilmaz et al 2013]. In view of this, scanning appointments were generally separated by 25 hours, and in practice, this regime worked well for both participants and MRI planning. According to previous work [Alam et al 2012], I chose a weight-adjusted USPIO dose of $4 \mathrm{mg} \mathrm{Fe} / \mathrm{kg}$ body weight. However, acknowledging that the distribution of USPIO following administration is predominantly in the organs of the reticuloendothelial system and blood pool, this may not be the optimum administration strategy as blood volume does not increase linearly with weight. I found a correlation between BMI and myocardial R2* change, probably due to increased blood pool USPIO concentration in those with a higher BMI. I therefore suggest that a fixed dose approach may also be appropriate depending on the application.

Artefacts were commonly encountered with USPIO-enhanced $\mathrm{T}^{*} *$ imaging and made data analysis challenging. Post contrast artefacts at the blood pool to myocardial interface were commonly seen and needed careful exclusion when selecting myocardial ROI (Figure 3.5). This limited the assessment of USPIO accumulation at the endocardium. Similarly, blooming artefacts from nearby organs with high iron or blood pool USPIO content, such as lung and liver, commonly created signal deficits within the myocardium. In this situation, examination of $\mathrm{T} 2 *$ 

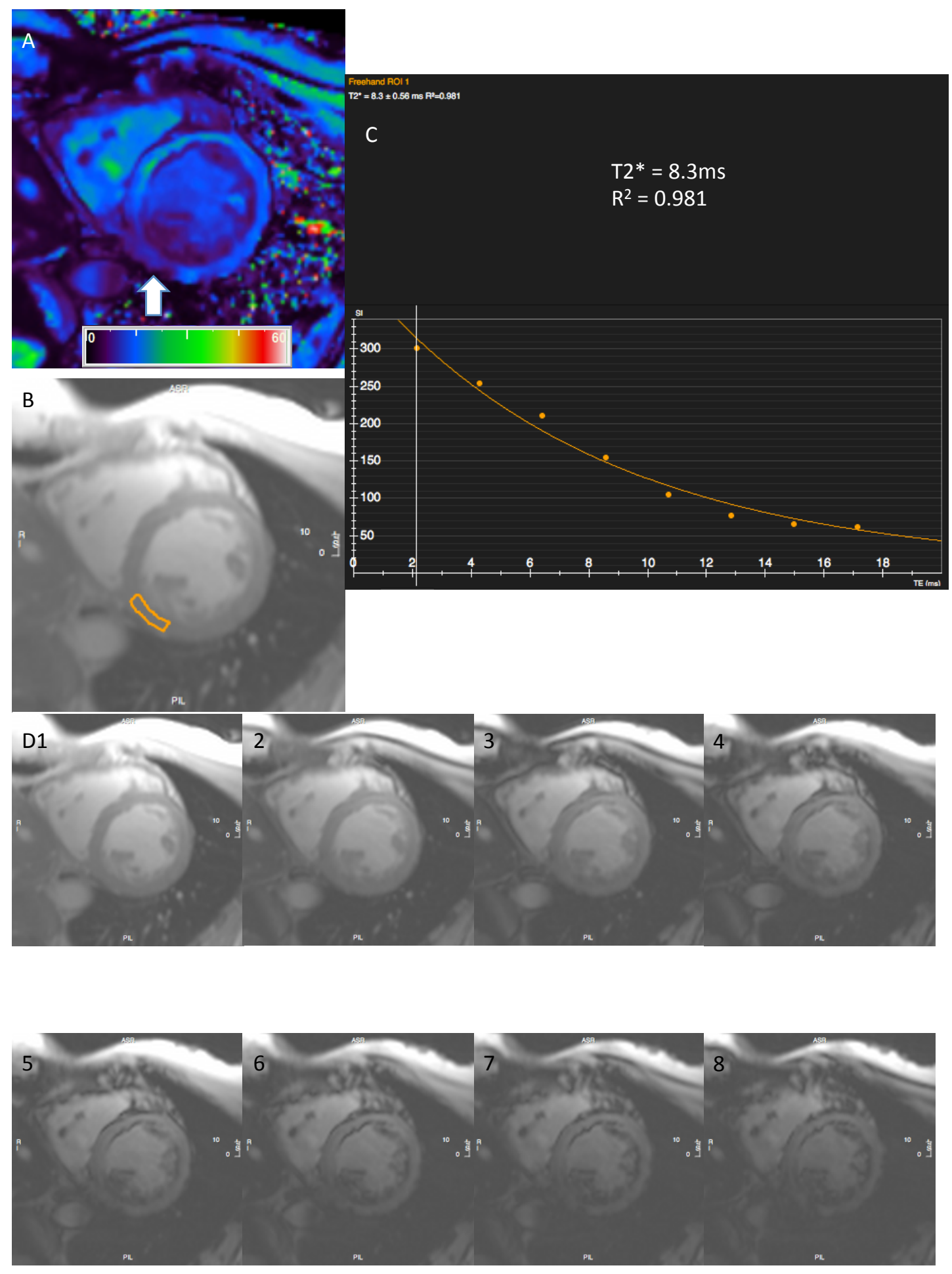

Figure 3.5 (A,B,C,D1-D8). 


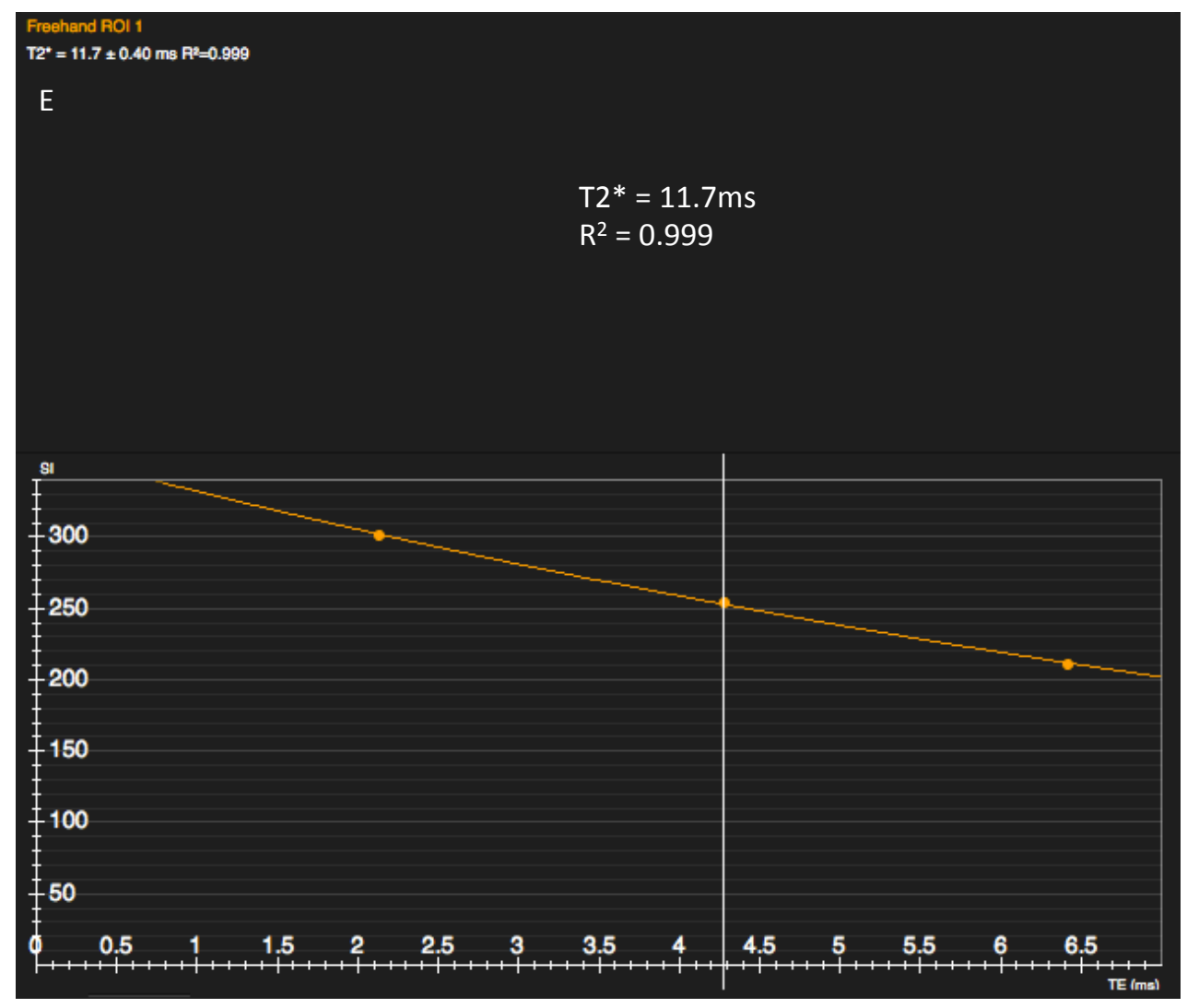

Figure 3.5. Inferior blooming artefact. Example illustrating the challenge in assessing whether the inferior myocardial signal attenuation seen arrowed on the T2* colourmap (A, scale 0-60 ms) is true or caused by artefact. Drawing a region of interest $(\mathbf{B})$ and examining the decay curve $(\mathbf{C})$ along with visualising individual echos (D1-8) helps determine that this is a 'blooming artefact' from outside the heart that affects echos 4-8. These can be manually removed, forming a new decay curve (E) with improvement in curve fitting $\left(\mathrm{r}^{2}\right.$ value), although with fewer fitting points. $\mathrm{r}^{2}-$ coefficient of determination. 
decay curves and excluding echo times influenced by artefact aided $\mathrm{T} 2 *$ curve fitting (Figure 3.5).

The advantage of MRI mapping techniques is that visual assessment and objective quantification can be made using the same image, and these are now entering clinical practice. It seems likely that if UPSIO-enhanced MR imaging is adopted into clinical practice to detect tissue inflammation, T2* mapping would be used for image interpretation. However based on my experiences, I would recommend caution in interpreting maps alone. Signal attenuation seen on the T2* map may be interpreted as tissue USPIO accumulation, but may be due to blooming artefact from nearby susceptibility effects, and close examination of the $\mathrm{T} 2 *$ decay curve, and individual echoes (if possible), is suggested in order to distinguish accurately between tissue USPIO accumulation and artefact. In theory, setting an $r^{2}$ threshold as we did helps to exclude areas grossly affected by artefact. In practice however, regions with a seemingly acceptable $r^{2}$ may still be influenced by artefact (Figure 3.5). Manual exclusion of later echoes (influenced by artefact) from the curve may result in an improvement in $r^{2}$ (a measure of how well the data points fit the curve), however there is the danger that reducing the number of fitting points will in fact reduce the overall sampling accuracy. Clearly, automated software capable of detecting and excluding artefact would be advantageous. This could be achieved by excluding, or applying less weight, to later echo times especially data points at a large distance from the initial decay curve trajectory [He et al 2007; Shah et al 2011]. It should be noted that like all other MRI sequences, poor data quality heavily influenced by 
breathing or movement artefact is generally non-interpretable and post processing using automated $\mathrm{T} 2 *$ decay curve fitting software is not likely to provide a remedy.

Echo times in this study were specific for cardiac imaging and were selected appropriately. Therefore they were not optimal for imaging tissues with $\mathrm{T} 2 *$ values substantially higher or lower than myocardium. Native blood pool and post USPIO bone marrow (Figure 3.6) provide examples of low and high $\mathrm{T} 2 *$ values respectively that I had difficulty accurately fitting a T2* decay curve. With high $\mathrm{T} 2 *$ values, only a short part of the decay curve is plotted over the echo sampling time period, and often the signal has not decayed sufficiently for an accurate decay curve to be plotted. In contrast, regions with particularly short $\mathrm{T} 2 *$ decay times have decayed to a level expected from background noise before sufficient data sampling has been made. Therefore fitting a decay curve from a small number (i.e. 2-4) of echo times is clearly difficult, and often too much emphasis is placed upon data decayed to the baseline level of background noise in order to generate a decay curve. Allowances can be made for background noise but are of limited value in this instance. I strongly advise applying tissue-specific echo times tailored to the expected $\mathrm{T} 2 *$ value in order to achieve the most accurate decay curves possible. 

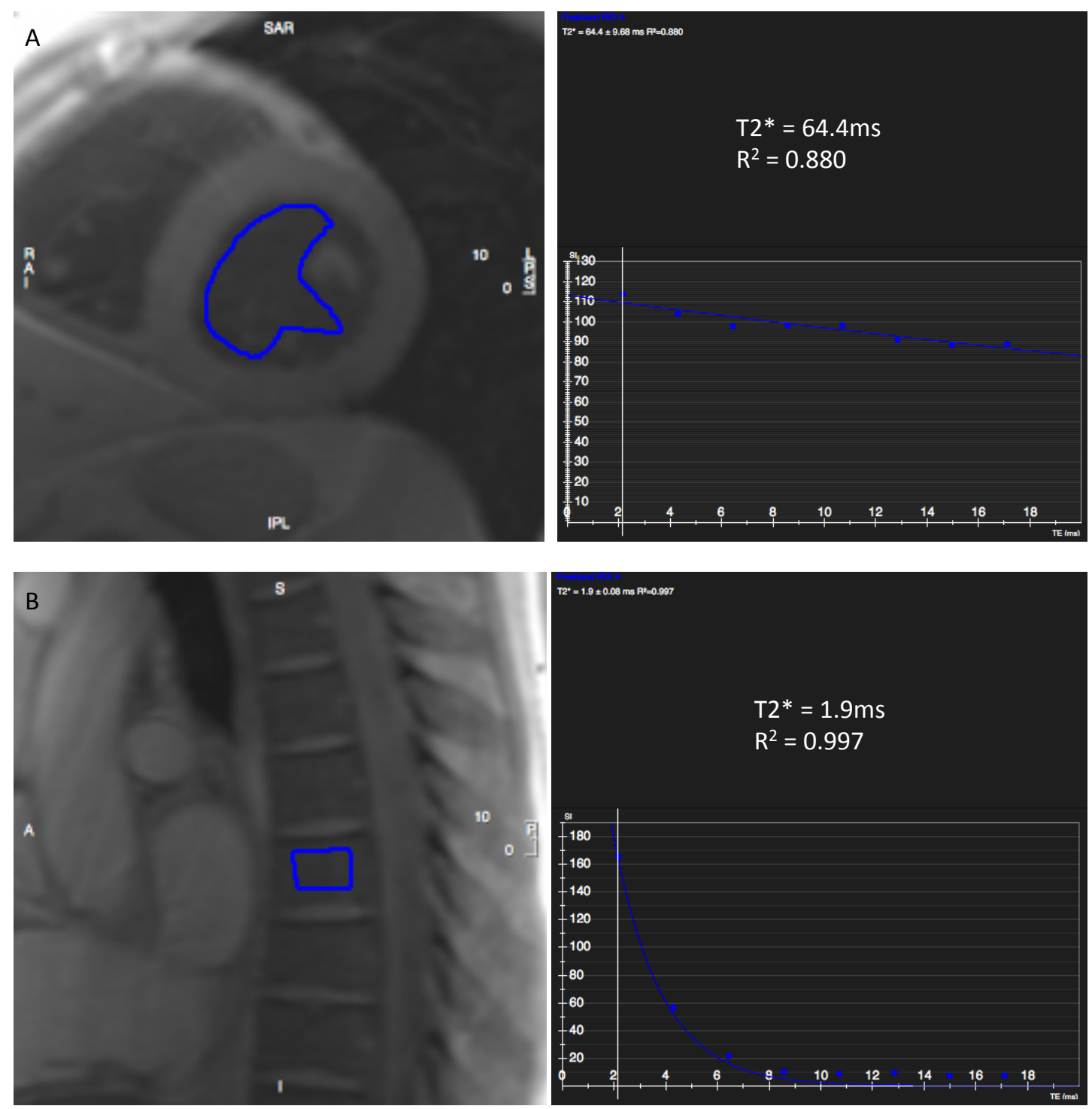

Figure 3.6. Example of high and low $\mathrm{T} 2 *$ values. Regions of interest with excessively low or high T2* value (pre-contrast blood pool, A) and post USPIO bone marrow, B), respectively) can often be difficult to generate an accurate $\mathrm{T} 2 *$ decay curve. Imaging with tissue specific echo times will help generate more accurate $\mathrm{T} 2 *$ decay curves. USPIO - ultrasmall superparamagnetic particles of iron oxide; $\mathrm{r}^{2}$ - coefficient of determination. 


\subsubsection{LiMitATIONS}

There are some limitations that should be taken into account when interpreting these data. First, this study has small numbers and a larger cohort of participants should be studied to further validate these normal values. Furthermore, due to geographical reasons, it was not feasible to scan the same participants at both centres so comparison cohorts at $1.5 \mathrm{~T}$ and $3 \mathrm{~T}$ were different. Despite this, both were healthy volunteers groups and displayed no differences at baseline so I do not feel this has impacted on the results. Finally, due to problems in interpreting high and low T2* values as mentioned above, I recommend caution in interpreting some high noncardiac R2* values; especially in the organs of the reticuloendothelial system at $3 \mathrm{~T}$. In these organs, the spread of $\mathrm{R} 2 *$ data above the median value appears wide. This is possibly caused by artefact and most evident at $3 \mathrm{~T}$, and may additionally explain why these regions have disproportionally high $\mathrm{R} 2 *$ values.

\subsubsection{CONCLUSION}

We have shown that ferumoxytol-enhanced MR imaging is feasible at both $1.5 \mathrm{~T}$ and $3 \mathrm{~T}$, and suggest a range of expected normal values post ferumoxytol in a range of tissues. Refinements of dose administration, optimisation of acquired echo times, careful image analysis, and development of post processing and analysis software capable of excluding common artefacts, are essential to ensure reliable and robust quantification of tissue enhancement. 


\section{CHAPTER 4}

\section{ULTRASMALL SUPERPARAMAGNETIC PARTICLES OF}

IRON OXIDE-ENHANCED T2* MAGNETIC RESONANCE IMAGING CAN BE USED TO DETECT AND SERIALLY MONITOR MYOCARDIAL INFLAMMATION AFTER MYOCARDIAL INFARCTION USING SINGLE AND REPEATED USPIO ADMINISTRATION 


\subsection{SUMMARY}

Objectives Macrophages play a central role in the cellular inflammatory response to myocardial infarction and predict subsequent clinical outcomes. I aimed to assess temporal changes in cellular inflammation and tissue oedema in patients with acute MI using ultrasmall superparamagnetic particles of iron oxide-enhanced magnetic resonance imaging.

Methods Thirty-one patients were recruited following acute myocardial infarction and followed up for 3 months with repeated T2 and USPIO-enhanced T2* mapping MR imaging. Regions of interest were categorised into infarct, peri-infarct, and remote myocardial zones, and compared to control tissues.

Results Following a single dose, USPIO enhancement was detected in the myocardium until 24 hours $(\mathrm{p}<0.0001)$. Histology confirmed colocalisation of iron and macrophages within the infarcted, but not the non-infarcted, myocardium. Following repeated doses, USPIO uptake in the infarct zone peaked at days 2-3, and greater USPIO uptake was detected in the infarct zone compared to remote myocardium until days $10-16(\mathrm{p}<0.05)$. In contrast, T2-defined myocardial oedema peaked at days 3-9 and remained increased in the infarct zone throughout the 3-month follow up period $(\mathrm{p}<0.01)$.

Conclusion Myocardial macrophage activity can be detected using USPIOenhanced magnetic resonance imaging in the first 2 weeks following acute myocardial infarction. This observed pattern of cellular inflammation is distinct, and provides complementary information to, the more prolonged myocardial oedema detectable, using T2 mapping. This imaging technique holds promise as a 
non-invasive method of assessing and monitoring myocardial cellular inflammation with potential application to diagnosis, risk stratification and assessment of novel anti-inflammatory therapeutic interventions.

\subsection{INTRODUCTION}

More than one person every minute suffers a myocardial infarction in the United States of America [Go et al 2014]. Despite improved interventional and medical treatments, mortality rates following MI remain high and many develop heart failure [Go et al 2014]. Post-infarct inflammation plays a key role in the recovery of cardiac function [Nahrendorf et al 2007; Nahrendorf et al 2010], and is a target for therapeutic manipulation to improve clinical outcomes.

After early neutrophil infiltration, monocyte-derived macrophages dominate the cellular infiltrate in the first 2 weeks following $\mathrm{MI}$ and sequentially coordinate digestion of damaged tissue and promotion of infarct healing [Nahrendorf et al 2007; Nahrendorf et al 2010]. Optimal post-MI recovery is determined by the balance between proinflammatory and reparative macrophages, with uncontrolled early inflammation leading to adverse functional recovery [Nahrendorf et al 2007; Tsujioka et al 2009; Nahendorf et al 2010; Panizzi et al 2010].

Ultrasmall superparamagnetic particles of iron oxide consist of an iron oxide core surrounded by a carbohydrate or polymer coating and are small enough to extravasate through diseased microvessels, where they are engulfed and concentrated 
by tissue-resident macrophages [Ruehm et al 2001]. Accumulation of USPIO reduces $\mathrm{T} 2 *$ decay time and creates signal deficits that can be quantified and visualised using $\mathrm{T} 2 *$ magnetic resonance imaging [Alam et al 2012; Yilmaz et al 2013]. Thus USPIO-enhanced MRI can detect tissue-resident macrophage activity and identify cellular inflammation within tissues.

In current practice, T2-weighted MRI is used to evaluate myocardial oedema after MI [Abdel-Aty et al 2004; Kellman et al 2007]. However these imaging techniques assess myocardial free water content and not active cellular inflammation. Development of a reliable non-invasive imaging technique capable of directly detecting myocardial cellular inflammation would be a major advance and could potentially facilitate risk stratification and therapeutic targeting of macrophages immediately after MI. Furthermore, this technique could provide diagnostic information, serial disease monitoring and a measure of treatment response in other conditions mediated by inflammatory cell infiltration of the heart.

In a recent proof-of-principle pilot study, Alam et al [2012], successfully detected inflammation following acute MI using USPIO-enhanced MRI. USPIO uptake, defined as the increase in $\mathrm{R} 2 *(1 / \mathrm{T} 2 *) 24$ hours following administration, was seen in the infarct zone within the heart. In this present study, I hypothesised that I could track the time course of cellular inflammation in the 3-month period following acute MI using USPIO-enhanced MRI. My primary aims were firstly to determine the duration and distribution of USPIO enhancement in tissues following a single infusion. Secondly, I aimed to assess the duration of macrophage infiltration of the 
heart by tracking myocardial USPIO enhancement using repeated USPIO administration, and compare this to measures of myocardial oedema using $\mathrm{T} 2$ mapping.

\subsection{METHODS}

This was an open label observational cohort study. Patients were recruited within 7 days of acute myocardial infarction. The study was performed in accordance with the Declaration of Helsinki, the approval of the Local Research Ethics Committee, and the written informed consent of all participants.

\subsubsection{SUBJECTS}

Participants were aged 18 to 80 years of age and had sustained a recent MI according to the Third Universal Definition of Myocardial Infarction [Thygesen et al 2012] with 12-hour plasma troponin I concentration $\geq 5000 \mathrm{ng} / \mathrm{L}$ (Architect STAT Troponin-I assay, Abbott Diagnostics). Exclusion criteria were known critical stenosis $(>95 \%)$ of the left main stem, ongoing symptoms of angina, heart failure (Killip class $\geq 2$ ), renal failure (estimated glomerular filtration rate $<30 \mathrm{~mL} / \mathrm{min} / 1.73$ $\mathrm{m}^{2}$ ), and contraindication to MRI or ferumoxytol infusion. Results were also compared to 'control', healthy myocardium at $3 \mathrm{~T}$, as described in the previous Chapter.

\subsubsection{USPIO}

Intravenous infusion of USPIO (ferumoxytol, $4 \mathrm{mg} / \mathrm{kg}$; Rienso ${ }^{\circledR}$ ) was performed 
immediately following the baseline magnetic resonance scan over at least 15 minutes using a concentration of $2-8 \mathrm{mg} / \mathrm{mL}$, diluted in $0.9 \%$ saline or $5 \%$ dextrose. Haemodynamic monitoring was conducted throughout.

\subsubsection{STUdY PROTOCOL}

A variable study protocol was used that was purposely designed for imaging at different time points after acute MI. Over a 3-month period following MI, patients received up to 7 MRI scans and up to 3 infusions of USPIO. Two principal analyses were performed using data from the same participants.

First, to assess the duration of a single dose of USPIO, R2* values of only the participants that first received USPIO within 7 days of MI (21/30) were included in this analysis. They were followed on all subsequent MRI scans prior to receiving any further doses of USPIO (Figure 4.1a). Upon receiving further USPIO, data were censored from the point of the second USPIO administration, hence the reduction in numbers over the course of the study.

Second, to assess the duration of myocardial macrophage activity, USPIO uptake was compared using repeated USPIO infusions over the 3-month follow up period. All patients were included in this analysis. USPIO uptake was calculated by subtracting the baseline R2* value from values obtained at 24 hours following USPIO infusion (Figure 4.1b). This allowed repeated assessment of myocardial USPIO uptake. 
Following clinical guidance for the administration of ferumoxytol, participants were allowed a maximum of two doses of USPIO in a 1-month period, and a total of three over the 3-month follow up period to prevent iatrogenic iron overload. Therefore, USPIO-enhanced data was collected up to three times for each patient and it was not possible to perform repeated USPIO-enhanced imaging at every time point in the same patient.

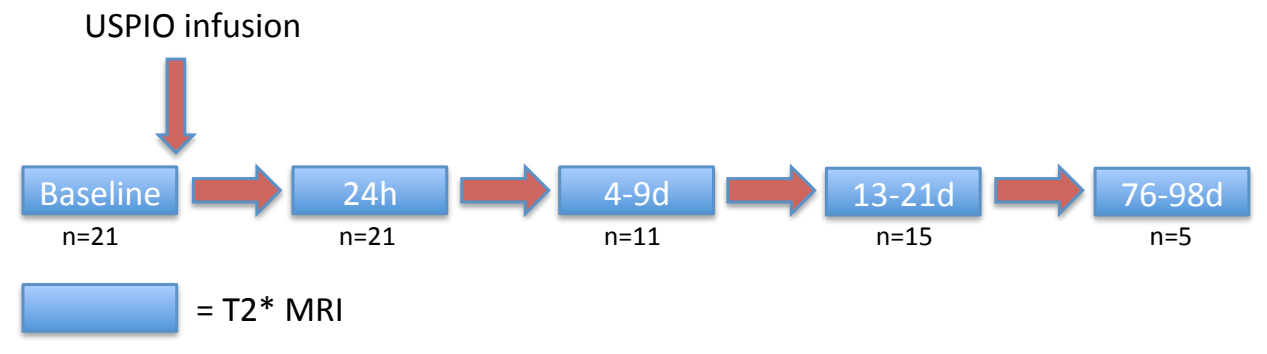

Figure 4.1a. Duration and distribution of USPIO enhancement after single-dose administration in the first week after MI. USPIO - ultrasmall superparamagnetic particles of iron oxide; $h$ - hours; d - days; MRI - magnetic resonance imaging; MI - myocardial infarction.

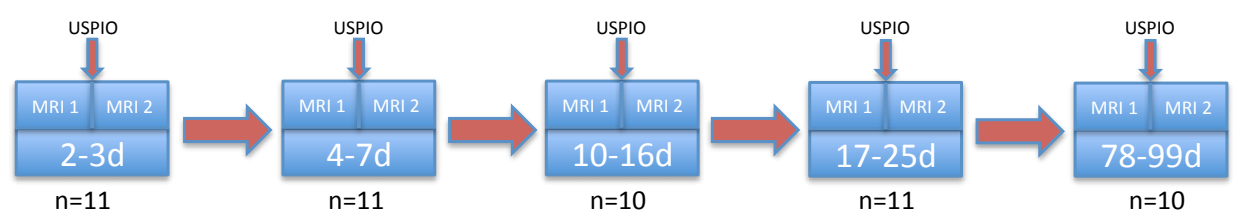

MRI $1=$ T2*, T2maps, wall motion, LGE

USPIO infusion immediately following MRI 1

MRI $2=\mathrm{T} 2 * 24 \mathrm{~h}$ after USPIO

Figure 4.1b. Repeated myocardial uptake of USPIO following MI. USPIO - ultrasmall superparamagnetic particles of iron oxide; MRI - magnetic resonance imaging; $d$ - days; LGE - late gadolinium enhancement; MI - myocardial infarction. 
Finally, to assess myocardial oedema, T2 values were compared on unenhanced scans (prior to and at least 7 days following USPIO administration). All patients were included in this analysis.

\subsubsection{MAGNETIC RESONANCE IMAGING PROTOCOL}

MR imaging was performed using a $3 \mathrm{~T}$ scanner and a Siemens 32-channel cardiac array. All images were acquired with electrocardiogram gating using expiration breath-holds. Routine steady state free precession sequences were used to acquire long and short axis cine images of the heart (TR $85.8 \mathrm{~ms}$, TE $1.45 \mathrm{~ms}$, flip angle $50^{\circ}$, matrix 173x256, FoV $400 \mathrm{~mm}$, slice thickness $8 \mathrm{~mm}, 2 \mathrm{~mm}$ gap). Quantitative USPIO imaging was performed again in similar slice positions using a prototype T2*-weighted multi-gradient-echo acquisition using a volumetric shim applied over the entire heart volume (TR $996 \mathrm{~ms}$, TE 2.13, 4.3, 6.4, 8.6, 10.7, 12.8, 15.0, $17.1 \mathrm{~ms}$, flip angle $18^{\circ}$, matrix 130x256, FoV $400 \mathrm{~mm}$, slice thickness $6 \mathrm{~mm}, 4 \mathrm{~mm}$ gap). The $\mathrm{T} 2 *$-weighted acquisitions included views through the liver, spleen and spine to allow quantification of USPIO accumulation within organs of the reticuloendothelial system. The same $\mathrm{T} 2 *$ protocol was used to quantify USPIO accumulation 24 hours after infusion allowing calculation of $\mathrm{T} 2 *$ relaxation rates before and after administration of USPIO. Oedema imaging was conducted using a Siemens T2 mapping based on a prototype T2-prepared TrueFISP acquisition acquiring identical long and short axis slice positions (TR $219.3 \mathrm{~ms}$, TE $1.07 \mathrm{~ms}$, T2P durations 0, 25, $50 \mathrm{~ms}$, flip angle $70^{\circ}$, matrix 130x192, FoV $400 \mathrm{~mm}$, slice thickness $6 \mathrm{~mm}, 4 \mathrm{~mm}$ gap). T2P-TrueFISP images are acquired at intervals of at least $3 \mathrm{RR}$ intervals to 
allow for sufficient magnetisation recovery in between acquisitions.

Immediately after the baseline $\mathrm{T} 2$ and $\mathrm{T} 2 *$-weighted scan, participants received an intravenous administration of gadolinium contrast medium $(0.1 \mathrm{mmol} / \mathrm{kg})$ followed by breath held inversion recovery sequences in long axis and short axis planes to acquire late enhancement images. Gadolinium-enhanced imaging is the current gold standard for infarct detection using MR imaging. Optimal inversion time was determined on a slice-by-slice basis using standard late enhancement TI-scout protocols (TR $750 \mathrm{~ms}$, TE $2.61 \mathrm{~ms}$, flip angle $20^{\circ}$, matrix $173 \times 256$, FoV $400 \mathrm{~mm}$, slice thickness $9 \mathrm{~mm}, 1 \mathrm{~mm}$ gap). The inversion recovery late enhancement short axis slices were acquired using similar slice positions as the T2-oedema and T2*-weighted imaging.

\subsubsection{IMAGE ANALYSIS}

All T2*-weighted multi-gradient-echo images for each patient were analysed using Circle CVI software. An experimentally determined threshold used in previous work [Alam et al 2012] for the coefficient of determination $\left(\mathrm{r}^{2}>0.85\right)$ was used to exclude data that did not have an acceptable exponential decay when signal intensity was plotted against echo time. The relaxation rate, $\mathrm{R} 2 *$, is the inverse of the mean $\mathrm{T} 2 *$, and was calculated to assess the uptake of USPIO for each region of interest, where the higher the value, the greater the USPIO accumulation.

Late gadolinium enhancement, ventricular volume and functional analyses were determined manually using QMass software. 


\subsubsection{REGION OF INTEREST (ROI) SELECTION}

Late gadolinium enhancement images at the 3-month time point were used to determine the distribution and anatomy of the myocardial infarct, and served as reference images to which each series of $\mathrm{T} 2$ and $\mathrm{T} 2 *$ scans at each separate visit could be visually coaligned. This allowed myocardial ROIs to be selected on the reference short axis LGE images and manually applied to T2* images corresponding to (1) infarct zone (defined by the short axis slice showing greatest LGE volume), (2) peri-infarct zone ( $1 \frac{2}{2}$ segment width immediately adjacent to LGE), and (3) remote myocardium (at least $1 / 2$ segment width, opposite the infarct zone on the same short axis slice, and at least 1 full segment clear of any LGE). Other selected regions included (4) liver, (5) spleen, (6) bone marrow, (7) kidney, (8) blood pool, (9) aortic wall (inside arch) and (10) skeletal muscle.

T2 values were measured immediately prior to USPIO administration. USPIOenhanced data were collected 24 hours following USPIO administration.

\subsubsection{Histology}

Myocardial tissue samples were obtained from patients undergoing coronary artery bypass graft surgery for treatment of their coronary disease within 2 weeks of their index myocardial infarction. USPIO was administered 24 hours prior to the biopsy, and a trucut myocardial biopsy sample was taken from the infarcted area, guided by the distribution of LGE on MR imaging, during the procedure. The biopsy sample was fixed in formalin, embedded in paraffin, sectioned, and stained to look at 
architecture (haematoxylin and eosin), accumulation and distribution of USPIO (Prussian Blue) and macrophages (CD68). Patients that underwent CABG surgery were excluded from further MRI follow up.

\subsubsection{StatisticAL ANALYSIS}

All statistical analysis was performed with GraphPad Prism, version 6. To assess duration of USPIO enhancement in tissues following single administration in the first week after myocardial infarction, R2* values on subsequent MRI scans (prior to any further USPIO dosing) were compared using one-way analysis of variance with Tukey's post hoc test for multiple comparisons.

To assess variation and duration of myocardial inflammation, USPIO uptake (R2* increase from pre to 24 hours following USPIO) was compared using one-way ANOVA with Tukey's post hoc test for multiple comparisons (repeated measures where appropriate). As a further method to assess USPIO accumulation within the heart, post USPIO R2* 24 hours after administration (without baseline R2* subtraction) was compared in the same way.

Finally, to assess variation and duration of myocardial oedema, T2 values for infarct, peri-infarct and remote myocardium were compared again using one-way ANOVA with Tukey's post hoc test for multiple comparisons (repeated measures where appropriate). Statistical significance was defined as two-sided $\mathrm{p}<0.05$. 


\subsection{RESULTS}

Thirty-one patients were recruited although one patient was excluded due to claustrophobia. One hundred and forty-seven MRI scans and 54 infusions of ferumoxytol were completed over the course of the study. Administration of ferumoxytol was well tolerated with no adverse reactions reported during or immediately after administration.

Participants were predominantly middle-aged males with current or previously diagnosed hypercholesterolaemia or smoking habit (Table 4.1). Around half of participants were hypertensive and had a family history of premature coronary heart disease. Twenty-five patients had ST segment elevation myocardial infarction, 5 patients non ST segment elevation myocardial infarction, 26 underwent percutaneous coronary intervention and 4 coronary artery bypass graft surgery. The mean elevation in plasma troponin was $31,200 \mathrm{ng} / \mathrm{L}$.

\subsubsection{ENHANCEMENT AFTER SINGLE USPIO DOSE}

Twenty-one of the thirty participants received USPIO in the first week after MI and were included in this analysis. 


\section{TABLE 4.1 Participant Characteristics}

Number of Participants 30

$\begin{array}{lc}\text { Age (years) } & 58.4 \pm 9.8 \\ \text { Sex } & \text { Male - } 29 \\ \text { Weight (kg) } & \text { Female } \\ & 88.7 \pm 14.8 \\ \text { Risk Factors } & \\ \text { Hypertension } & \\ \text { Diabetes mellitus } & 13(43) \\ \text { Hypercholesterolaemia } & 8(27) \\ \text { Family history } & 27(90) \\ \text { Current or ex-smoker } & 14(47) \\ \end{array}$

\section{Infarct Characteristics}

STEMI

Anterior

$10(33)$

Inferior

$15(50)$

Lateral

$0(0)$

Diagnostic ECG to reperfusion (STEMI, min)

$72.5 \pm 16.8$

NSTEMI

PCI

$26(87)$

Baseline ejection fraction (\%)

$48.5 \pm 11.2$

Infarct size ( $\%$ of LV mass)

$31.6 \pm 19.5$

Infarct volume $(\mathrm{mL})$

$43.9 \pm 27.5$

Plasma troponin $(\mathrm{ng} / \mathrm{L})$

$31,229 \pm 18,563$

Subsequent CABG/Biopsy

4/ 3

mean \pm SD, N (\%).

STEMI - ST elevation myocardial infarction; NSTEMI - non ST elevation myocardial infarction; PCI percutaneous coronary intervention; LV - left ventricular; CABG - coronary artery bypass graft; SD - standard deviation. 
Ultrasmall superparamagnetic particles of iron oxide were detected by an increase in $\mathrm{R} 2 *$ in all myocardial regions, blood pool, kidney and aortic wall until 24 hours, being cleared by 4-9 days (Figure 4.2 and Table 4.2). USPIO were detected in bone marrow until days 4-9, and liver and spleen until days 13-21. There was no change in R2* following USPIO infusion in skeletal muscle.

Myocardial biopsies were taken from 3 of 4 patients undergoing $\mathrm{CABG}$ and revealed an abundance of inflammatory cells and early granulation tissue in keeping with a region of healing myocardium from around the infarct zone. Histological staining revealed colocalisation of the presence of iron (Prussian Blue) and macrophages (CD68). No staining for iron or macrophages was seen within the adjacent regions of healthy myocardium (Figure 4.3).

\subsubsection{TIME COURSE OF ENHANCEMENT WITH REPEATED USPIO ADMINISTRATION}

There were no differences in baseline (pre USPIO) R2* value between infarct and remote myocardium at all time points. Time course variation in USPIO uptake was seen in the infarct zone over the 3-month period, peaking at days 2-3 (Figure 4.4a) and (Table 4.3a). There was no time course variation in USPIO uptake in the periinfarct, remote myocardium or extracardiac tissues over the 3-month follow up period. 

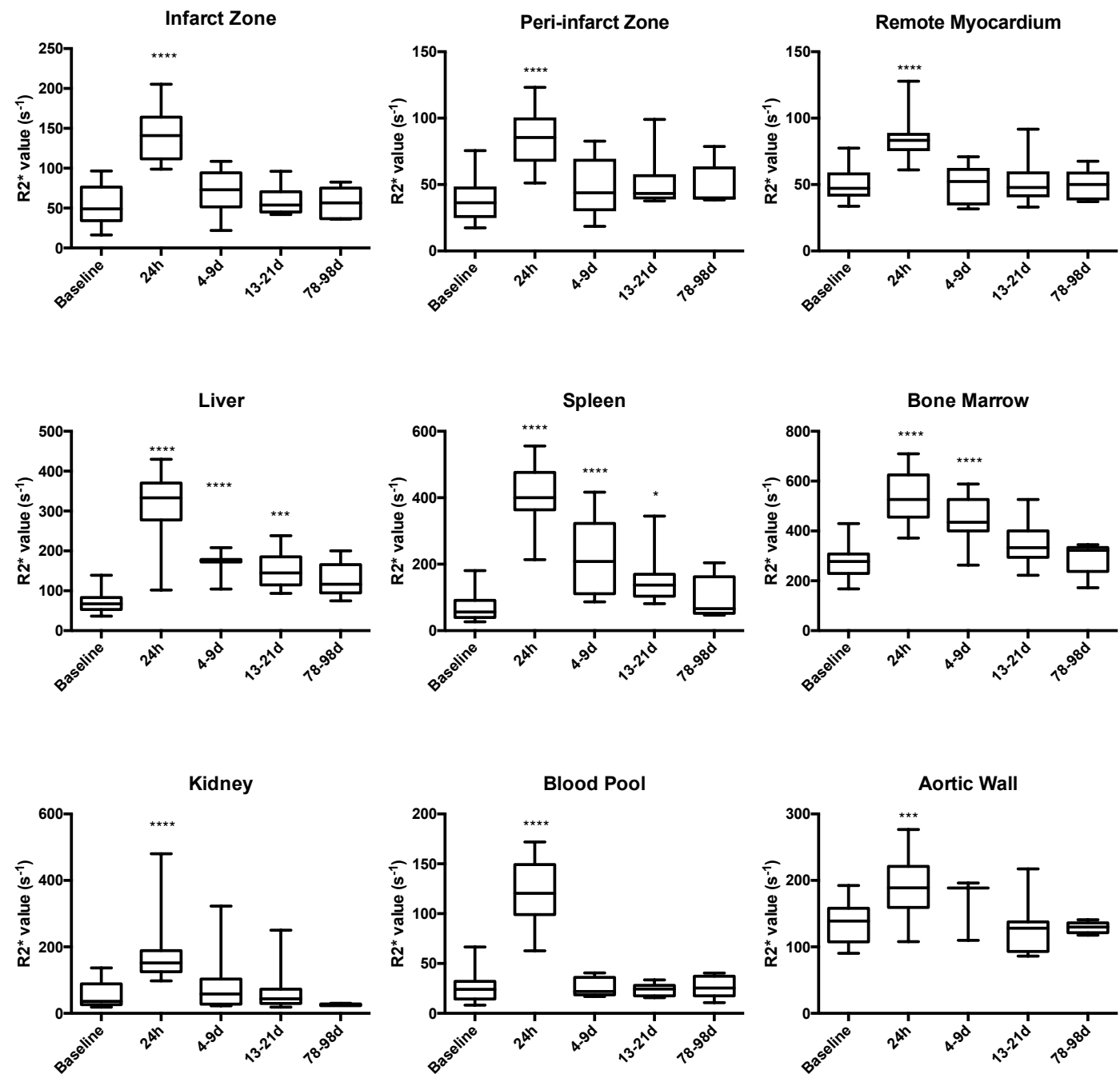

Skeletal Muscle

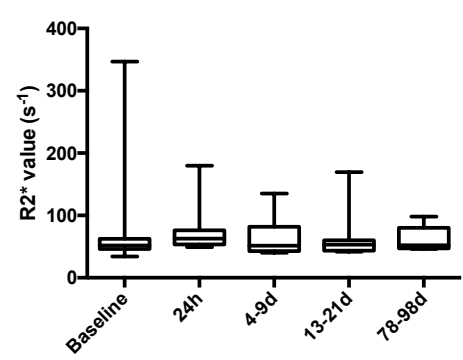

Figure 4.2. USPIO enhancement after single-dose administration in the first week following MI. Following single-dose administration, USPIO enhancement was detected in all myocardial regions, kidney, blood pool and aortic wall at 24 hours. USPIO was detected in bone marrow until days 4-9, and spleen and liver until days 13-21. There was no change in R2* in skeletal muscle following USPIO infusion $(\mathrm{p}>0.05)(* * * *=$ $\mathrm{p}<0.0001, * * *=\mathrm{p}<0.001, *=\mathrm{p}<0.05$ ). $\mathrm{n}=21$ (baseline, $24 \mathrm{~h}) 11$ (days 4-9), 15 (days 13-21), and 5 (days 78-99). USPIO - ultrasmall superparamagnetic particles of iron oxide; MI - myocardial infarction; $\mathrm{h}$ - hours; d - days; MI - myocardial infarction. 

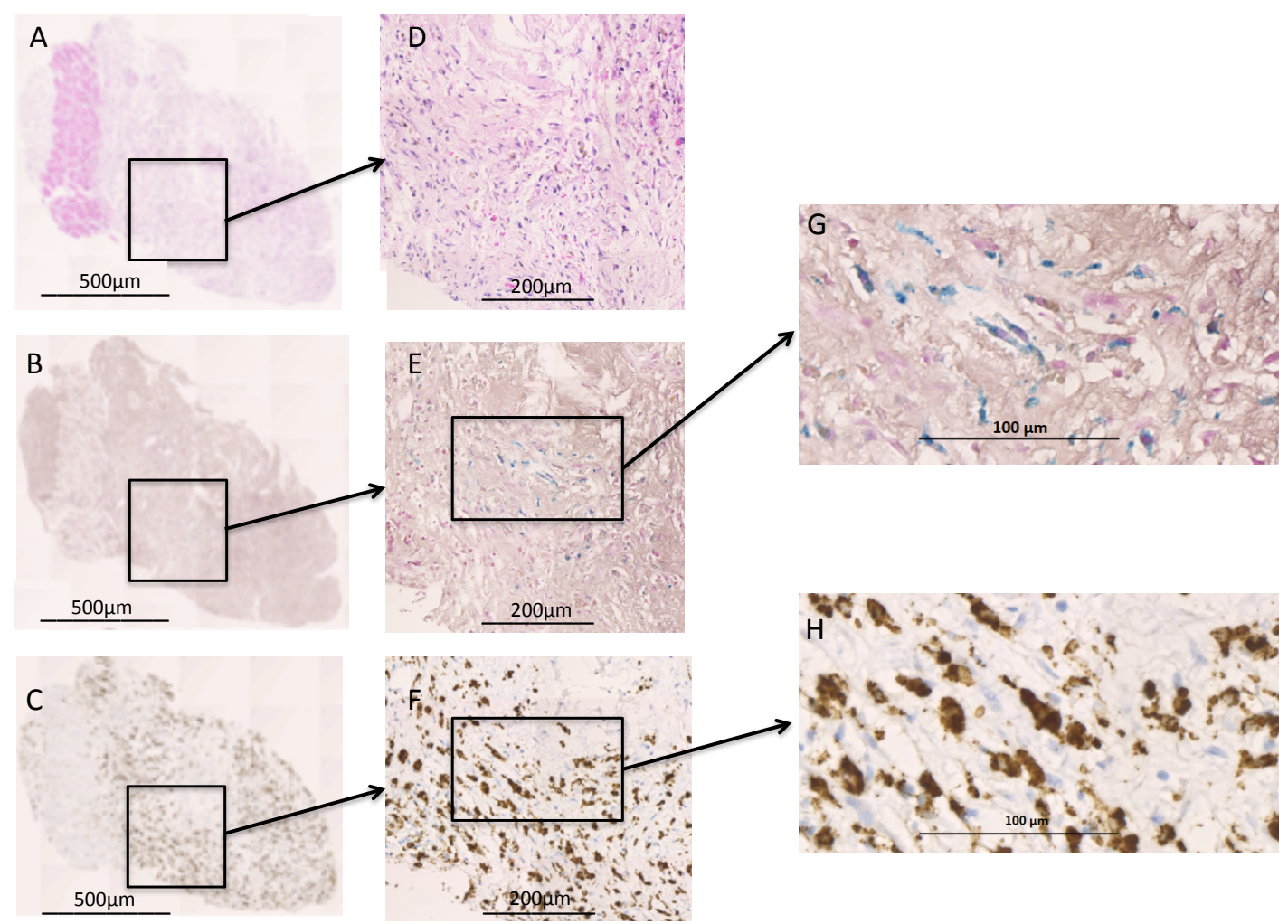

Figure 4.3. Histology section of trucut biopsy from infarcted myocardium. A) Haematoxylin and eosin (x5 magnification) stain displaying a thin region of viable healthy myocardium (strip of dark pink) surrounded by infarcted myocardium (lighter pink). Infarcted myocardium shows an abundance of inflammatory cells and early granulation formation (D). B) Prussian Blue (x5) staining revealing intracellular iron $(\mathbf{E} \times 20$ and $\mathbf{G} \times 50)$, not seen in the region of healthy myocardium. $\mathbf{C})$ CD68 (x5) staining revealing macrophages within the infarcted myocardium ( $\mathbf{F} \times 20$ and $\mathbf{H} \times 50)$, again not seen in healthy myocardium. $\mathrm{G}+\mathrm{H}$ show colocalisation of iron within macrophages. $\mathrm{CD}-\mathrm{cluster}$ of differentiation. 


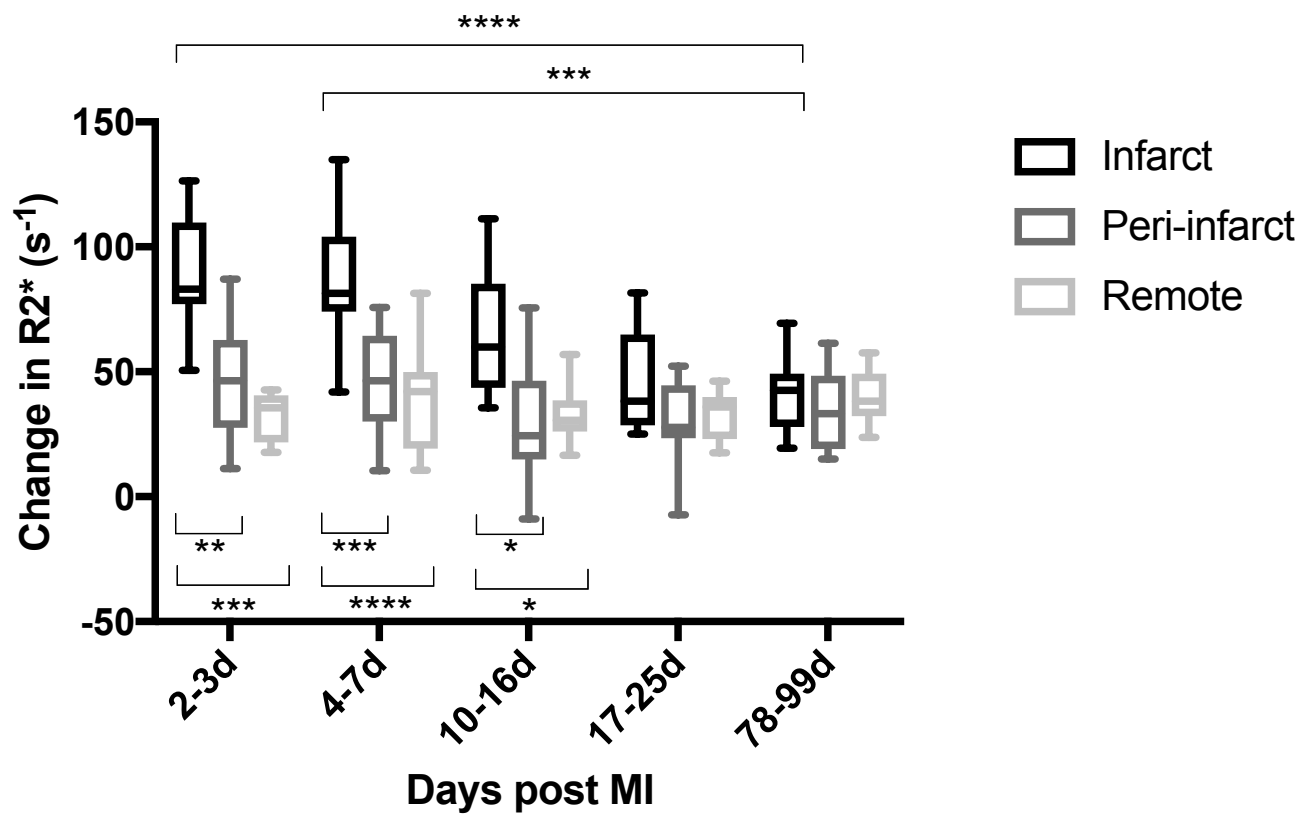

Figure 4.4a. USPIO uptake in myocardium after MI with repeated USPIO administration. Time course variation in USPIO uptake was seen in the infarct zone peaking at days 2-3. No variation of USPIO uptake was seen in peri-infarct and remote myocardium. Compared to remote myocardium, increased USPIO uptake was seen in the infarct zone until days $10-16$ post MI $(* * * *=p<0.0001$, $* * *=p<0.001, * *=p<0.01 *=p<0.05)$. USPIO - ultrasmall superparamagnetic particles of iron oxide; MI - myocardial infarction; $\mathrm{d}$ - days.

Figure 4.4a+4.4b: $n=11$ (days 2-3, 4-7 and 17-25), 10 (days 10-16) and 10 (days 78-99).

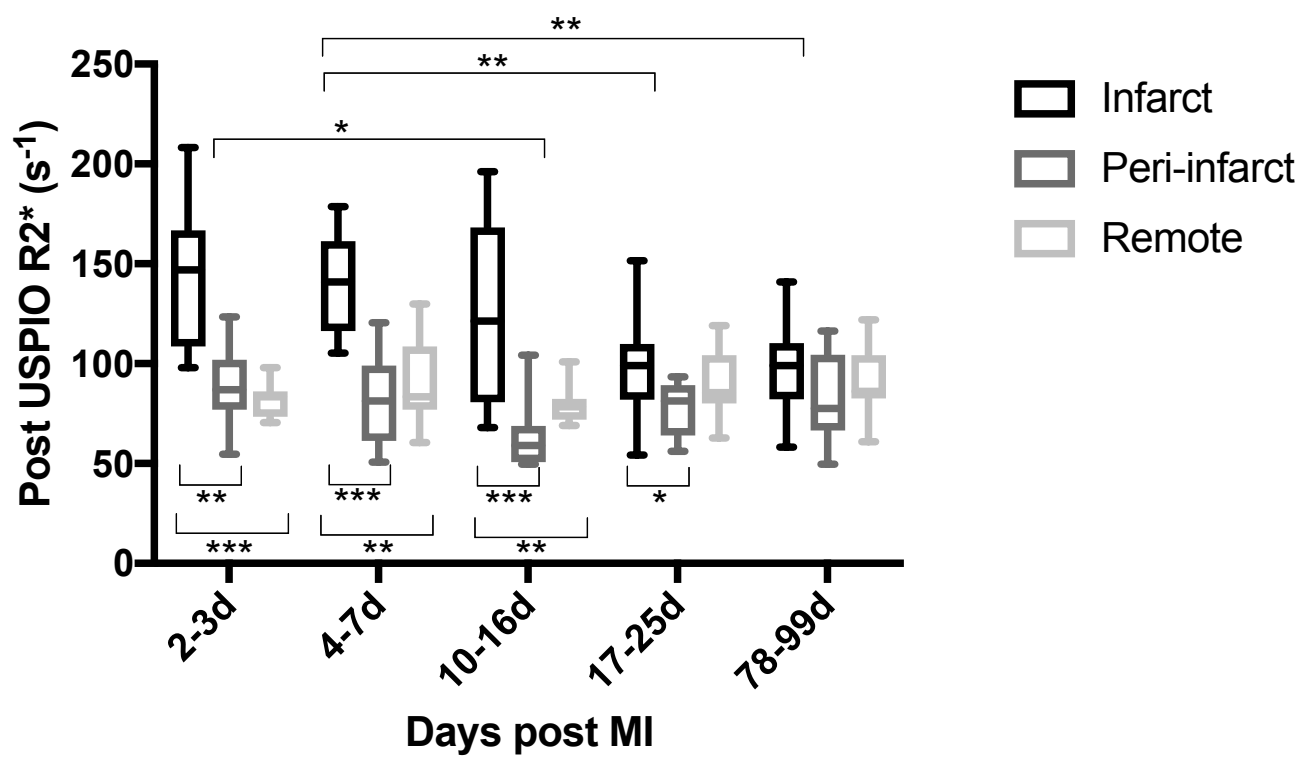

Figure 4.4b. R2* 24 hours following USPIO (without subtraction of baseline values). Compared to remote myocardium, greater post USPIO R2* was seen in the infarct zone until days 10-16 post MI $(* * *=\mathrm{p}<0.001, * *=\mathrm{p}<0.01, *=\mathrm{p}<0.05)$. USPIO - ultrasmall superparamagnetic particles of iron oxide; $\mathrm{d}$ - days; $\mathrm{h}$ - hours; MI - myocardial infarction.

Figure 4.4a+4.4b: $n=11$ (days 2-3, 4-7 and 17-25), 10 (days 10-16) and 10 (days 78-99). 
Compared to remote myocardium, greater USPIO uptake was seen in the infarct zone until days 10-16 (mean +56 seconds $(\mathrm{s})^{-1} ; 95 \%$ confidence interval (CI) 33 to 79 ; $\mathrm{p}<0.001,+47 \mathrm{~s}^{-1} ; 95 \%$ CI 31 to $62 ; \mathrm{p}<0.0001$, and $+33 \mathrm{~s}^{-1} ; 95 \%$ CI 4 to $61 ; \mathrm{p}<0.05$ at days 2-3, days 4-7 and days 10-16 respectively) but not thereafter (Figure 4.4a and Figure 4.5). Similarly, comparing R2* 24 hours following USPIO (without baseline R2* subtraction), greater USPIO uptake was again evident in infarcted myocardium compared to remote myocardium until days $10-16$ (mean $+60 \mathrm{~s}^{-1}$; $95 \%$ CI 33 to 86 ; $\mathrm{p}<0.01,+49 \mathrm{~s}^{-1} ; 95 \%$ CI 21 to $77 ; \mathrm{p}<0.01$, and $+45 \mathrm{~s}^{-1} ; 95 \%$ CI 2 to $87 ; \mathrm{p}<0.05$ at days 2-3, days 4-7 and days 10-16 respectively, (Figure 4.4b and Table 4.3b).

There was a negative correlation between infarct size and USPIO uptake within the infarct zone in the first 2 weeks of MI $(r=-0.51, p<0.01, n=26)$, particularly within the first 2-3 days $(\mathrm{r}=-0.73, \mathrm{p}<0.01, \mathrm{n}=11)$. Nine patients had imaging evidence of microvascular obstruction (MVO). There was no statistically significant difference in USPIO accumulation between the non-MVO group when compared to the MVO group (mean R2* change 94 vs. $79 \mathrm{~s}^{-1}, \mathrm{p}=0.14$ ).

\subsubsection{ASSESSMENT OF MYOCARDIAL OEDEMA USING T2 MAPPING}

Following acute MI, time course variation in infarct and peri-infarct T2 value was seen (Figures 4.5, 4.6 and Table 4.4). No time course variation in T2 value was seen in remote myocardium. Compared to remote myocardium, greater myocardial T2 value was detected in the infarct zone and remained present throughout the 3-month follow up period (mean $+12 \mathrm{~ms} ; 95 \%$ CI 7 to 18 ; $\mathrm{p}<0.001,+14 \mathrm{~ms} ; 95 \%$ CI 9 to 18 ; $\mathrm{p}<0.0001,+15 \mathrm{~ms} ; 95 \%$ CI 6 to $24 ; \mathrm{p}<0.01,+12 \mathrm{~ms} ; 95 \%$ CI 5 to $19 ; \mathrm{p}<0.01$, and 
$+6 \mathrm{~ms} ; 95 \%$ CI 2 to $9 ; \mathrm{p}<0.01$ at days $1-2$, days $3-9$, days $10-16$, days $17-24$ and days 77-98 respectively).

\subsubsection{TIME COURSE OF ENHANCEMENT WITH REPEATED USPIO ADMINISTRATION COMPARED WITH HEALTHY VOLUNTEERS (FROM CHAPTER 3)}

Compared to healthy volunteer myocardium, greater USPIO uptake was seen in the infarct zone until days 10-16 (mean +51 seconds $(\mathrm{s})^{-1} ; \quad 95 \%$ confidence interval (CI) 28 to $75 ; \mathrm{p}<0.0001,+49 \mathrm{~s}^{-1} ; 95 \%$ CI 26 to $73 ; \mathrm{p}<0.0001$, and $+28 \mathrm{~s}^{-1}$; $95 \%$ CI 4 to $52 ; \mathrm{p}<0.05$ at days $2-3$, days $4-7$ and days $10-16$ respectively) but not thereafter (Table 4.5). No significant difference in USPIO uptake existed between peri-infarct or remote myocardial infarction tissue and healthy volunteer control tissue at any of the time points. 
LGE
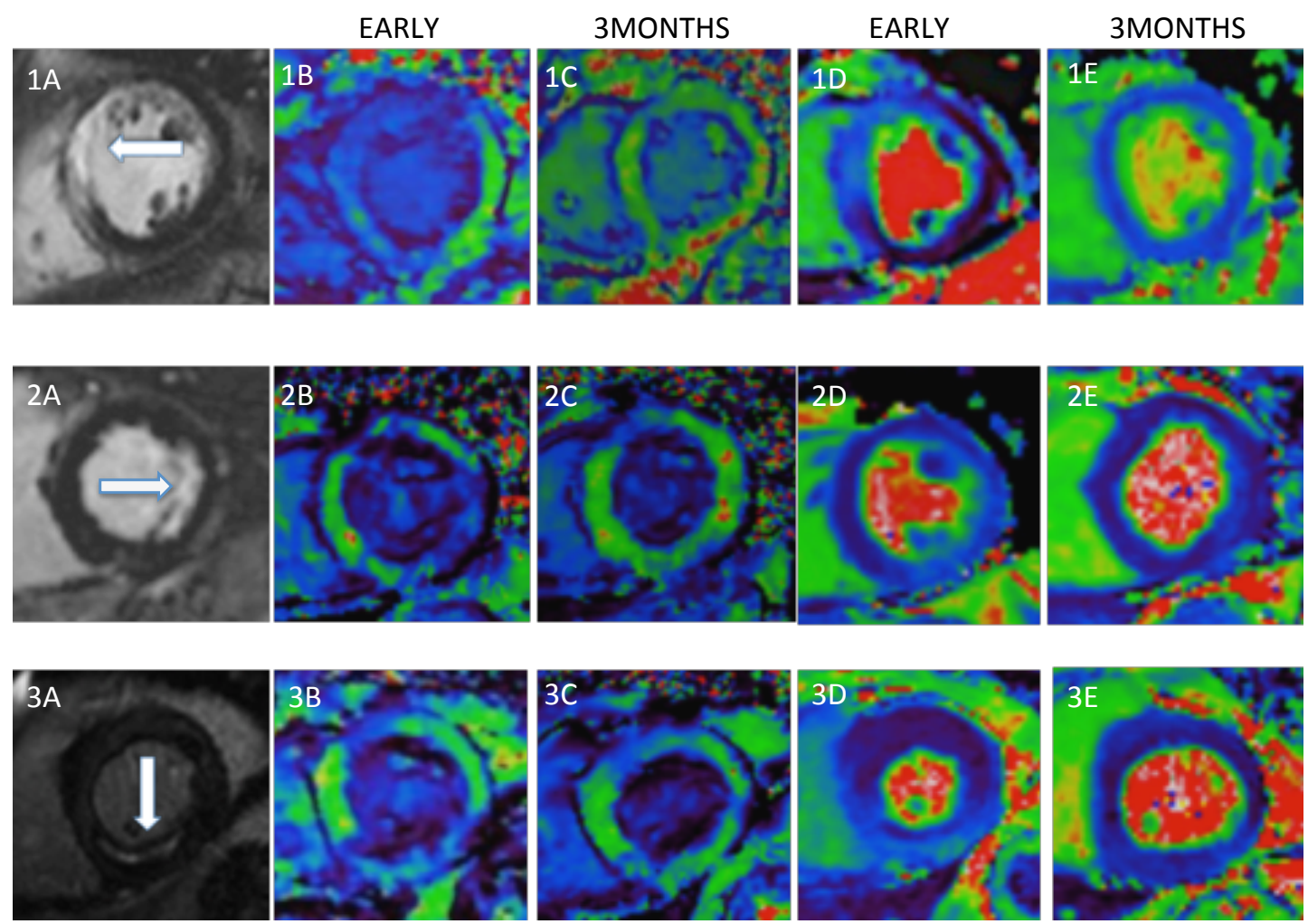

T2 map

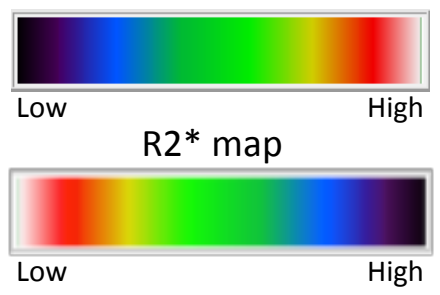

Figure 4.5. Examples of myocardial oedema and USPIO enhancement in the infarct zone after MI. Three examples of MI (1: anteroseptal, 2: lateral and 3: inferior) illustrating LGE, USPIO enhancement (R2* map) and oedema (T2 map), at early (up to 10 days) and late (3 months) time points. Early inflammation and oedema seen on R2* (dark region) and T2 maps (light region) respectively has improved or resolved by 3 months. LGE - late gadolinium enhancement; USPIO ultrasmall superparamagnetic particles of iron oxide; MI - myocardial infarction. 


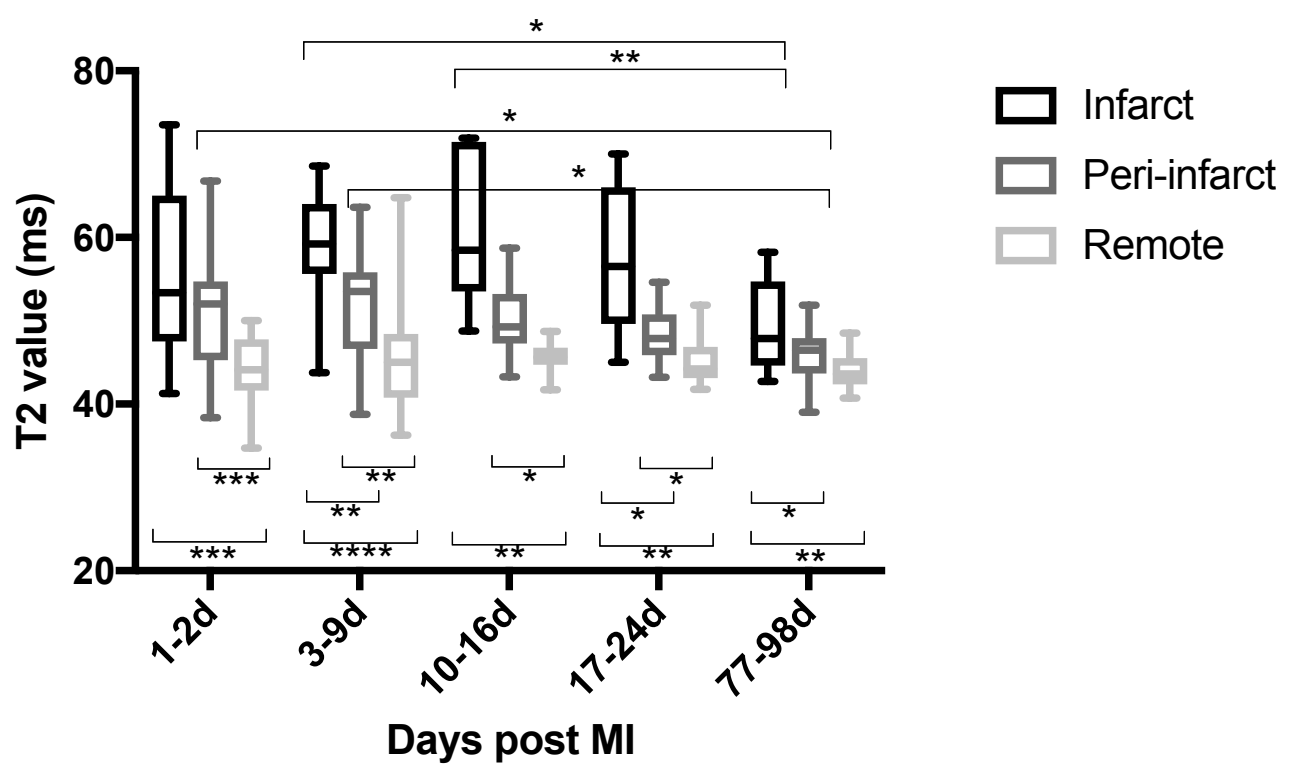

Figure 4.6. T2 value in the myocardium after MI. Time course variation in infarct and peri-infarct T2 was seen in the 3-month period following MI, both peaking at days 3-9. Compared to remote myocardium, higher T2 was seen in the infarct zone throughout the duration of the study, and in the peri-infarct zone until days 17-24 $(* * * *=\mathrm{p}<0.0001, * * *=\mathrm{p}<0.001, * *=\mathrm{p}<0.01, *=\mathrm{p}<0.05)$. $\mathrm{n}=15$ (days 1-2), 14 (days 3-9 and 77-98), 8 (days 10-16) and 10 (days 17-24). MI - myocardial infarction; $\mathrm{d}$ - days. 
TABLE 4.2 USPIO enhancement after single-dose administration in the first week following MI

\begin{tabular}{|c|c|c|c|c|c|}
\hline & Baseline & $24 \mathrm{~h}$ & 4-9d & 13-21d & 78-99d \\
\hline Infarct & $49[34-76]$ & 141 [112-164] & 73 [52-94] & $54[45-70]$ & $57[37-75]$ \\
\hline Peri-infarct & 36 [25-49] & $85[67-101$ & 44 [30-69] & $43[39-58]$ & 40 [39-64] \\
\hline Remote & 47 [41-59] & 83 [75-89] & 52 [34-63] & $48[40-60]$ & $50[38-60]$ \\
\hline Liver & $67[53-83]$ & 333 [278-370] & 175 [172-178] & 145 [115-185] & 116 [95-166] \\
\hline Spleen & 56 [40-91] & $400[364-476]$ & 208 [111-323] & 137 [104-170] & 66 [52-162] \\
\hline Bone marrow & 278 [230-308] & 526 [456-625] & $435[400-526]$ & 333 [294-400] & 323 [238-334] \\
\hline
\end{tabular}

TABLE 4.3a USPIO uptake (change in $\mathrm{R}^{*}, \mathrm{~s}^{-1}$ ) in myocardium after MI with repeated USPIO administration

\begin{tabular}{|c|c|c|c|c|c|}
\hline & $2-3 d$ & $4-7 d$ & 10-16d & $17-25 d$ & 78-99d \\
\hline Infarct & 83 [77-110] & 81 [74-104] & 60 [44-85] & 38 [29-65] & 43 [28-49] \\
\hline Peri-infaret & 46 [28-63] & $46[30-64]$ & $24[15-46]$ & $28[23-45]$ & 33 [19-48] \\
\hline Remote & 36 [22-41] & 42 [19-50] & 31 [26-39] & $36[23-40]$ & 38 [32-49] \\
\hline
\end{tabular}

TABLE 4.3b R2* $\left(\mathrm{s}^{-1}\right) 24$ hours following USPIO (without subtraction of baseline values)

\begin{tabular}{|c|c|c|c|c|c|}
\hline & $2-3 d$ & $4-7 d$ & $10-16 d$ & $17-25 d$ & 78-99d \\
\hline Infarct & 147 [109-167] & 141 [116-161] & $121[81-168]$ & 99 [82-110] & 99 [82-110] \\
\hline Peri-infarct & 87 [77-102] & 81 [61-99] & 59 [51-69] & 81 [64-89] & 78 [67-105] \\
\hline Remote & 83 [74-86] & 83 [77-109] & 78 [72-82] & 85 [80-104] & 86 [83-104] \\
\hline & $1-2 d$ & 3-9d & $10-16 d$ & $17-24 d$ & 77-98d \\
\hline Infarct & 53 [48-65] & 59 [56-64] & 58 [54-71] & 57 [50-66] & $48[45-55]$ \\
\hline Peri-infarct & $52[45-55]$ & $54[47-56]$ & 49 [47-53] & $48[46-51]$ & $46[44-48]$ \\
\hline $\begin{array}{l}\text { Remote } \\
\text { median [IQR] }\end{array}$ & $44[42-48]$ & $45[41-48]$ & 46 [45-47] & $44[43-47]$ & $44[42-46\}$ \\
\hline
\end{tabular}


TABLE 4.5 USPIO uptake (change in $\mathrm{R} 2 *, \mathrm{~s}^{-1}$ ) in myocardium after MI with repeated USPIO administration compared to Healthy Volunteers (Chapter 3 )

\begin{tabular}{|c|c|c|c|c|c|}
\hline & $2-3 d$ & $4-7 d$ & 10-16d & $17-25 d$ & 78-99d \\
\hline Infarct & $83[77-110]^{* * * *}$ & $81[74-104] * * * *$ & $60[44-85]^{*}$ & 38 [29-65] & 43 [28-49] \\
\hline Peri-infarct & $46[28-63]$ & $46[30-64]$ & $24[15-46]$ & $28[23-45]$ & 33 [19-48] \\
\hline Remote & $36[22-41]$ & 42 [19-50] & 31 [26-39] & $36[23-40]$ & 38 [32-49] \\
\hline Healthy Control & $35[31-38]$ & $35[31-38]$ & $35[31-38]$ & $35[31-38]$ & $35[31-38]$ \\
\hline \multicolumn{6}{|c|}{$\begin{array}{l}\text { Compared to healthy control myocardium, increased USPIO uptake was seen in the infarct zone until } \\
\text { days } 10-16 \text { post MI }(* * * *=p<0.0001, *=\mathrm{p}<0.05) \text {. There is no significant difference in USPIO } \\
\text { uptake between peri-infarct or remote myocardial infarction tissue and healthy volunteer control tissue } \\
\text { at any of the time points. }\end{array}$} \\
\hline \multicolumn{6}{|l|}{ median $[\mathrm{IQR}]$} \\
\hline
\end{tabular}




\subsection{DISCUSSION}

For the first time, I have simultaneously investigated cellular inflammation and tissue oedema for 3 months following acute myocardial infarction using USPIO-enhanced $\mathrm{T} 2 *$ and $\mathrm{T} 2$ mapping magnetic resonance imaging. I have demonstrated that whilst tissue oedema persists for at least 3 months, cellular inflammation in the infarct zone is transient and lasts for only 2 weeks following MI. The time course of USPIO uptake following MI demonstrates a pattern of macrophage inflammation that is distinct to tissue oedema caused by loss of capillary integrity. This suggests that whilst capillary integrity may take several months to resolve, cellular inflammation and specifically macrophage activity predominates in the first 2 weeks following myocardial infarction.

Ultrasmall superparamagnetic particles of iron oxide can be used as an MRI contrast agent as it is immediately apparent in the blood pool following intravenous administration [Taylor et al 1999]. Tissue enhancement with USPIO following inflammation has been suggested to occur as a result of either partitioning of USPIO into the tissues because of loss of capillary integrity alone or as a result of macrophage phagocytic activity clearing tissue resident particles [Yilmaz et al 2013]. I here provide confirmatory evidence that USPIO enhancement predominantly occurs as a result of cellular inflammation within the myocardium. I used T2 mapping to describe loss of capillary integrity following myocardial infarction. Using this comparator, I demonstrated that there is a discontinuity between the prolonged (up to 3 months) T2-defined time course of tissue oedema, and the brief 
(first 2 weeks) T2*-defined time limited cellular inflammation. This observed USPIO uptake in the infarct zone for up to 2 weeks following MI is consistent with data from previous studies demonstrating that tissue-resident macrophages are active and predominate within the infarcted myocardium in the first 2 weeks after MI [Tsujioka et al 2009; Nahrendorf et al 2010]. Additionally, I obtained myocardial biopsies in patients undergoing coronary artery bypass graft surgery within 2 weeks of myocardial infarction and 24 hours after USPIO administration. I demonstrated marked colocalisation of iron staining with tissue-resident macrophages in infarcted but not non-infarcted tissue. It is not possible to resolve specific USPIO on histology and I cannot discount the possibility that there is some contribution from post-infarct hemosiderin staining. However, I would contend that the majority of iron staining is attributable to USPIO uptake given the selective, transient and marked R2* enhancement on MRI, and the relatively constant contribution from hemosiderin over the period of assessment. Moreover, I did not detect substantial quantities of iron that were free in the extracellular space and were not associated with areas of macrophage infiltration.

Following a single infusion in the first week after MI, USPIO enhancement is seen in all regions of the myocardium, but greatest within the infarct zone, after 24 hours. USPIO enhancement is also observed until days 4-9 in bone marrow, and until days 13-21 in the liver and spleen. Therefore the duration of USPIO enhancement is longest in the mononuclear phagocyte rich reticuloendothelial system. It is likely that resident phagocytes within these organs ingest USPIO from circulating blood. Efflux of myocardial macrophages containing USPIO may also contribute to the 
reticuloendothelial system $\mathrm{T} 2 *$ signal. Ultimately, once within macrophages, the iron oxide core is broken down by lysosomes, providing a supply of iron ions for haemoglobin synthesis [Van Beers et al 2001; Thorek et al 2006].

The observed duration of enhancement following a single infusion of USPIO in tissues helped guide the minimum time interval between repeated USPIO administrations. Complete washout of the previous dose of USPIO, allowing baseline $\mathrm{R} 2 *$ to return to normal, is required to reassess uptake of USPIO accurately, without influence from previous USPIO administration. I have shown that USPIO is no longer detectable in the myocardium at 1-week following intravenous administration and I suggest that 1-week is the minimum interval between repeated doses of USPIO.

USPIO is detectable in the peri-infarct and remote myocardium, kidney and aortic wall at 24 hours following administration. However the amplitude of R2* change is less than that of blood pool, suggesting this is likely to be due to a diluted effect of blood pool USPIO and not macrophage uptake of USPIO within these tissues. Although there appears a trend to suggest that the peri-infarct zone displays an early relative increase in USPIO uptake compared to remote myocardium, this failed to reach statistical significance.

Previous studies have suggested that the remote myocardium may exhibit USPIO and macrophage accumulation [Alam et al 2012; Lee et al 2012; Yilmaz et al 2013]. I have here shown that there is no time course variation in USPIO uptake in remote myocardium after myocardial infarction and the small R2* increase following 
USPIO is likely to be reflective of intravascular capillary bed blood pool USPIO rather than more widespread macrophage activity throughout the myocardium. This is confirmed with no significant difference between USPIO uptake in remote myocardium and healthy control myocardium (Chapter 3 and Table 4.5).

Although no patients in this study displayed imaging evidence of myocardial haemorrhage, I was able to account for the presence of microscopic iron due to haemorrhage by subtracting baseline $\mathrm{R} 2 *$. Therefore, any $\mathrm{R} 2 *$ increase detected is due to accumulation of USPIO and not iron from myocardial haemorrhage. I acknowledge that intramyocardial haemorrhage occurring between the pre and 24 hours post USPIO scans could theoretically increase R2*, but the earliest I imaged patients was at least 24 hours after myocardial infarction, by which time haemorrhage is likely to have taken place.

The weak negative correlation between infarct size and USPIO uptake within the infarct zone in the first 2 weeks of MI is interesting and deserves mention. This was likely driven by microvascular obstruction in patients with large infarcts that prevented USPIO accessing infarcted tissue. However when we compared subgroups of patients with and without MVO, although no statistically significant difference in USPIO uptake existed, there may be a suggestion that the MVO group had less USPIO uptake. This study lacked power to detect differences in USPIO uptake between subgroups of patients with and without MVO. An increased sample size will help determine whether a difference in USPIO uptake between these groups truly exists. 
I chose T2 mapping to assess myocardial oedema as it provides improved sensitivity and addresses some of the problems with subjective image interpretation using traditional T2-weighted techniques [Giri et al 2009; Verhaert et al 2011]. The finding of ongoing myocardial oedema in the infarct zone at 3 months after MI may at first appear surprising. However several groups have described the long lasting presence of myocardial oedema, often up to 6 months after MI [Nilsson 2001; Dall'Armellina et al 2011]. This suggests that it may take many months for complete resolution of injury and return of normal vascular homeostasis and function.

What are the clinical applications of my technique? I have here used the 'model' of acute myocardial infarction and demonstrated the predicted time course of tissueresident macrophage uptake using USPIO. I therefore suggest that this technique has utility in defining the extent of myocardial cellular inflammation that has potential utility in risk stratification. Perhaps more importantly, USPIO-enhanced MRI may prove a useful tool to assess the response to novel anti-inflammatory interventions targeted at reducing myocardial cellular inflammation to improve clinical outcomes. Such a study was carried out at our centre and has recently been published [Alam et al 2015]. Currently, there is no USPIO licensed as a contrast agent for routine clinical MR imaging, which limits its widespread use. However the most exciting application would be to extend this technique to help diagnose, monitor disease progression and assess therapeutic intervention in myocardial diseases associated with and mediated by macrophage infiltration. Such diseases include myocarditis, cardiac transplant rejection and cardiac sarcoidosis and a multicentre study is 
currently underway to assess this (EUDRACT 2013-002336-24). This would be important given the lack of simple definitive diagnostic tests in these conditions, the challenges of unreliable cardiac biopsies, and the need for rapid assessments of disease progression and therapeutic interventions.

In conclusion, I have successfully imaged, quantified and validated macrophage inflammation and myocardial oedema over 3 months in patients following MI using USPIO-enhanced $\mathrm{T} 2 * \mathrm{MR}$ imaging and $\mathrm{T} 2$ mapping. This technique can provide a non-invasive method for the diagnosis and monitoring of tissue inflammatory macrophage activity in the heart. It also provides a potential platform on which to assess existing and novel therapeutic interventions that might modify the inflammatory process, not only after MI but also in other inflammatory processes affecting the heart. 


\section{CHAPTER 5}

FERUMOXYTOL-ENHANCED MAGNETIC RESONANCE IMAGING IN ACUTE MYOCARDITIS 


\subsection{SUMMARY}

Background Ultrasmall superparamagnetic particles of iron oxide-enhanced magnetic resonance imaging can detect tissue-resident macrophage activity and identify cellular inflammation within tissues. I hypothesised USPIO-enhanced MRI would provide a non-invasive imaging technique that would improve the diagnosis and management of patients with acute myocarditis.

Methods Ten volunteers and 14 patients with suspected acute myocarditis underwent $\mathrm{T} 2, \mathrm{~T} 2 *$ and late gadolinium enhancement $3 \mathrm{~T}$ MR imaging, with further T2* imaging at 24 hours after USPIO (ferumoxytol, $4 \mathrm{mg} / \mathrm{kg}$ ) infusion, at baseline and 3 months. Myocardial oedema and USPIO enhancement were determined within areas of LGE as well as throughout the myocardium.

Results Myocarditis was confirmed in 9 of the 14 suspected cases of myocarditis. There was greater myocardial oedema in regions of late gadolinium enhancement in patients with myocarditis when compared to healthy volunteer myocardium (T2 value, $57.1 \pm 5.3$ versus $46.7 \pm 1.6 \mathrm{~ms}$; $\mathrm{p}<0.0001$ ). There was no demonstrable difference in USPIO enhancement between patients and volunteers even within regions displaying LGE (change in $\mathrm{R} 2 * 3,35.0 \pm 15.0$ versus $37.2 \pm 9.6 \mathrm{~s}^{-1} ; \mathrm{p}>0.05$ ). Imaging after 3 months in myocarditis patients revealed a reduction in volume of LGE, a reduction in oedema measures within regions displaying LGE, and improvement in ejection fraction (LGE mean $-19.7 \mathrm{~mL} ; 95 \%$ CI -0.5 to $-40.0 \mathrm{~mL}$, $\mathrm{T} 2-5.8 \mathrm{~ms} ; 95 \% \mathrm{CI}-0.9$ to $-10.7 \mathrm{~ms}$, and $\mathrm{EF}+6 \%$; $95 \%$ CI 0.5 to $11.5 \%$ respectively; $\mathrm{p}<0.05$ for all). 
Conclusion In patients with acute myocarditis, USPIO-enhanced MRI does not provide additional clinically relevant information to LGE and T2 mapping MRI. This suggests that tissue-resident macrophages do not provide a substantial contribution to the myocardial inflammation in this condition.

\section{$5.2 \quad$ INTRODUCTION}

Acute myocarditis comprises a wide clinical spectrum from subclinical disease to severe heart failure, and is a major cause of sudden death in young adults [Drory et al 1991]. Pathologically, it is characterised by inflammatory cell infiltration of the myocardium with evidence of myocyte necrosis that is not characteristic of an ischaemic aetiology [Aretz et al 1987]. Causes of myocarditis include infections, immune-mediated injury, and toxins (such as anthracyclines) although frequently no cause is identified [Caforio et al 2013].

A variety of inflammatory cells infiltrate the myocardium during myocarditis. In viral myocarditis, the infiltrate is predominantly lymphocyte rich, but other cells including plasma cells, neutrophils, eosinophils, giant cells, and macrophages are also present [Aretz et al 1987; Feldman and McNamara 2000; Magnani and Dec 2006; Li et al 2009; Barin et al 2012; Sagar et al 2012; Jaquenod et al 2015]. Monocytes differentiate into macrophages at sites of myocarditis, suggesting they play an important role in the injury or repair of the myocardium [Baba et al 2006]. 
Endomyocardial biopsy is considered the gold standard investigation for diagnosis. However this is prone to sampling error and is uncommon in routine clinical practice [Caforio et al 2013]. Cardiac magnetic resonance imaging plays a key role in the diagnosis and shows typical appearances on T2-weighted and LGE imaging according to the Lake Louise Criteria [Friedrich et al 2009]. More recently, quantitative mapping techniques appear to improve diagnostic accuracy further [Luetkens et al 2015].

Iron oxide nanoparticles are generating interest as an MRI contrast agent able to detect macrophages, and clinical applications are now emerging [Trivedi et al 2004; Tang et al 2006; Trivedi et al 2006; Tang et al 2009; Richards et al 2011; Alam et al 2012; Yilmaz et al 2013; McBride et al 2015; Stirrat et al 2016 and 2017]. Ultrasmall superparamagnetic particles of iron oxide consist of an iron oxide core surrounded by a carbohydrate or polymer coating. They are small enough to extravasate passively through capillaries, where they are engulfed by tissue-resident macrophages [Ruehm et al 2011], and are detectable by T2*-weighted MR imaging. Thus USPIO-enhanced MRI can identify tissue-resident macrophage activity and identify cellular inflammation within tissues.

In a promising preclinical study of autoimmune myocarditis [Moon et al 2012], iron nanoparticles were ingested by inflammatory cells and improved distinction of areas with severe inflammation on MRI compared to conventional T2-weighted and gadolinium-enhanced MRI. I aimed to assess and to quantify myocardial USPIO enhancement in acute myocarditis, and to correlate enhancement with clinical 
measures of inflammation and oedema using T2 mapping and LGE MR imaging and compare these measures with healthy volunteers [Stirrat et al 2016]. I hypothesised that USPIO-enhanced MRI would detect myocardial macrophage activity in myocarditis and provide a cellular specific non-invasive imaging technique that may aid and improve patient diagnosis and management.

\subsection{METHODS}

Patients were recruited from a single centre as part of an open label observational multicentre cohort study (NCT02319278). The study was performed in accordance with the Declaration of Helsinki, the approval of the Scotland A Research Ethics Committee, and the written informed consent of all participants. The Medicines and Healthcare products Regulatory Agency of the United Kingdom gave Clinical Trial Authorisation for the study (EUDraCT 2013-002336-24).

\subsubsection{STUDY POPULATIONS}

Adult ( $>18$ years of age) patients with suspected acute myocarditis were recruited into the study. The clinical diagnosis was made by an independent clinical cardiologist based on the history, electrocardiogram, serum troponin (ARCHITECT STAT troponin I assay, Abbott Diagnostics), and other available imaging modalities. The diagnosis of myocarditis was verified from casenote review by an independent cardiologist and retained for analysis if the MRI scan (reported and agreed by two independent radiologists) showed imaging features of myocarditis on LGE and/or T2 mapping [Friedrich et al 2009]. Healthy volunteers had no clinically significant past 
medical history. Exclusion criteria were contraindication to MRI or ferumoxytol infusion, any other inflammatory comorbidity, renal failure (estimated glomerular filtration rate $<30 \mathrm{~mL} / \mathrm{min} / 1.73 \mathrm{~m}^{2}$ ), pregnancy, breastfeeding and women of childbearing potential without reliable contraception.

\subsubsection{STUDY PROTOCOL}

Patients with suspected myocarditis and healthy volunteers underwent paired MRI scans at baseline, and patients were invited to return for repeat imaging after 3 months.

\subsubsection{MAGNETIC RESONANCE IMAGING}

Magnetic resonance imaging was performed using a Magnetom Verio $3 \mathrm{~T}$ MRI scanner running software version VB17, with a dedicated cardiac array coil. All images were acquired using electrocardiogram-gated breath-holds. Routine steady state free precession sequences were used to acquire long axis and short axis images of the heart. Oedema imaging was conducted using a Siemens T2 mapping based on a prototype T2-prepared TrueFISP acquisition acquiring identical long and short axis slice positions. Quantitative USPIO imaging was performed in similar slice positions using a prototype $\mathrm{T} 2 *$-weighted multi-gradient-echo acquisition using a volumetric shim applied over the entire heart volume. Standard cardiac slice widths $(6 \mathrm{~mm}$ width with $4 \mathrm{~mm}$ gap) and 8 echo times (2.1-17.1 ms range) with matrix size of $256 \times 115$ were acquired for $\mathrm{T} 2 *$ maps. The in-plane resolution differed as required for larger or smaller objects; generally, a field of view of $400 \times 300 \mathrm{~mm}$ was used 
with an in-plane resolution of $2.6 \times 1.6 \mathrm{~mm}$. T2* relaxation times were calculated before and after administration of USPIO.

Immediately after the baseline T2, T2* and SSFP cine imaging, breath held inversion recovery sequences in long axis and short axis planes were used to acquire late enhancement images following an intravenous administration of gadolinium contrast medium $(0.1 \mathrm{mmol} / \mathrm{kg})$. Optimal inversion time was determined on a slice-by-slice basis using standard late enhancement TI-scout protocols. The inversion recovery late enhancement short axis slices were acquired using similar slice positions as the $\mathrm{T} 2$ and $\mathrm{T} 2 *$ imaging.

\subsubsection{USPIO}

Intravenous infusion of USPIO (ferumoxytol, $4 \mathrm{mg} / \mathrm{kg}$ ) was performed immediately following the baseline magnetic resonance scan over 15 minutes using a concentration of $2-8 \mathrm{mg} / \mathrm{mL}$, diluted in $0.9 \%$ saline or $5 \%$ dextrose. Haemodynamic monitoring was conducted throughout.

\subsubsection{IMAGE ANALYSIS}

All T2*-weighted multi-gradient-echo images for each patient were analysed using Circle CVI software. An experimentally determined threshold used in previous work [Alam et al 2012] for the coefficient of determination $\left(\mathrm{r}^{2}>0.85\right)$ was used to exclude data that did not have an acceptable exponential decay when signal intensity was plotted against echo time. Individual images affected by artefact were excluded. The inverse of the mean $\mathrm{T} 2 *(\mathrm{R} 2 *)$ for each $\mathrm{ROI}$ was then calculated to assess the uptake 
of USPIO, where the higher the value, the greater the USPIO accumulation.

T2 maps, late gadolinium enhancement, ventricular volume and functional analyses were also performed using Circle CVI software. T2 and T2* data were collected immediately prior to USPIO administration. USPIO-enhanced $\mathrm{T}^{*}$ data were collected 24-25 hours following ferumoxytol administration.

\subsubsection{REGION OF INTEREST (ROI) SELECTION}

Regions of interest were drawn using the standard 16-segment cardiac model [Cerqueira et al 2002], and pan-myocardial values averaged using all 16 segments. As a final method of analysis to focus on inflamed myocardium, regions with contiguous LGE $>1 \mathrm{~cm}^{2}$ on a single short axis slice were retained and visually coaligned with corresponding T2 and T2* images. These corresponding coaligned regions were then averaged to form $\mathrm{LGE}+\mathrm{T} 2$ and $\mathrm{T} 2 *$ regions.

\subsubsection{Statistical ANALYSIS}

All statistical analysis was performed with GraphPad Prism, version 6. Shapiro-Wilk normality testing was carried out prior to testing. To compare USPIO uptake and myocardial oedema in patients and volunteers, $\mathrm{R} 2 *$ and $\mathrm{T} 2$ values were compared using paired and unpaired $t$-tests, Mann-Whitney, and Wilcoxon tests depending on pairing and normality of data. To compare results at 3 months with baseline, a paired $t$-test was used. Statistical significance was defined as two-sided $\mathrm{p}<0.05$. 


\subsection{RESULTS}

Ten volunteers and 14 patients with suspected myocarditis were recruited. Nine patients had confirmed myocarditis according to imaging criteria and independent review. Baseline imaging was conducted within a week of diagnosis. Four patients had alternative diagnoses (tako-tsubo cardiomyopathy $(n=2)$, lung cancer $(n=1)$, polymyositis $(\mathrm{n}=1))$ and 1 patient had an incidental finding of an unknown chest wall metallic implant. These 5 patients were excluded from further analysis. All 9 patients retained for analysis had typical features of myocarditis on late gadolinium enhancement imaging. Of these 9 patients, 1 patient did not return at baseline for the 24 hour post USPIO scan. Seven of the 9 patients returned at 3 months for repeat imaging.

The healthy volunteer group was older with greater ejection fraction than the myocarditis group at baseline $(\mathrm{p}<0.001$ for both; Table 5.1). Patients with myocarditis had greater neutrophil count, CRP and serum troponin concentrations than volunteers $(\mathrm{p}<0.05, \mathrm{p}<0.01$ and $\mathrm{p}<0.0001$ respectively). There were no other significant differences between participant groups at baseline.

Administration of ferumoxytol was well tolerated with no adverse reactions reported during or immediately after administration in any of the participants. 


\begin{tabular}{|c|c|c|c|}
\hline & $\begin{array}{c}\text { Healthy } \\
\text { Volunteers }\end{array}$ & $\begin{array}{l}\text { Myocarditis } \\
\text { (Baseline) }\end{array}$ & $\begin{array}{l}\text { Myocarditis } \\
\text { (3 months) }\end{array}$ \\
\hline Number & 10 & 9 & 7 \\
\hline Female & 6 & 1 & 1 \\
\hline Age (years) & $50(45-53)$ & $28(24-34) * * *$ & $25(23-35)$ \\
\hline Body mass index $\left(\mathrm{kg} / \mathrm{m}^{2}\right)$ & $26(23-29)$ & $25(22-29)$ & $25(22-27)$ \\
\hline $\begin{array}{l}\text { Left ventricular ejection } \\
\text { fraction }(\%)\end{array}$ & $61.1 \pm 4.1$ & $51.0 \pm 4.9 * * *$ & $57.1 \pm 4.3$ \\
\hline $\begin{array}{l}\text { Late gadolinium } \\
\text { enhancement }(\mathrm{mL})\end{array}$ & Nil & $30.3 \pm 19.3$ & $16.3 \pm 12.3$ \\
\hline $\begin{array}{l}\text { ECG } \\
\text { ST elevation } \\
\text { T wave inversion } \\
\text { Normal }\end{array}$ & $\mathrm{n} / \mathrm{a}$ & $\begin{array}{c}9 / 9 \\
7 \\
1 \\
1\end{array}$ & \\
\hline $\begin{array}{l}\text { Coronary Angiogram } \\
\text { Normal }\end{array}$ & $\mathrm{n} / \mathrm{a}$ & $\begin{array}{c}4 / 9 \\
4\end{array}$ & \\
\hline $\begin{array}{l}\text { Echo } \\
\text { Wall motion } \\
\text { abnormality } \\
\text { Normal }\end{array}$ & $\mathrm{n} / \mathrm{a}$ & $\begin{array}{c}8 / 9 \\
4 \\
4\end{array}$ & \\
\hline $\begin{array}{l}\text { Blood Tests } \\
\text { White cell count }\left(\times 10^{9} / \mathrm{L}\right) \\
\text { Neutrophil count }\left(\times 10^{9} / \mathrm{L}\right) \\
\text { Lymphocyte count }\left(\times 10^{9} / \mathrm{L}\right) \\
\text { Monocyte count }\left(\times 10^{9} / \mathrm{L}\right) \\
\text { C-reactive protein }(\mathrm{mg} / \mathrm{L}) \\
\text { Troponin }(\mathrm{ng} / \mathrm{L})\end{array}$ & $\begin{array}{l}5.8(4.2-7.1) \\
3.2(2.2-4.2) \\
1.7(1.4-2.1) \\
0.4(0.3-0.7) \\
1.0(1.0-2.5) \\
2.0(1.5-4.0)\end{array}$ & $\begin{array}{c}7.1(6.1-8.9) \\
4.4(3.7-5.2)^{*} \\
1.8(1.3-2.2) \\
0.6(0.5-0.8) \\
38(14-63)^{* *} \\
11842(3950- \\
25722)^{* * * *}\end{array}$ & $\begin{array}{l}5.1(4.6-5.6) \\
2.8(2.7-3.5) \\
1.8(1.2-1.9) \\
0.4(0.3-0.5) \\
2.0(1.0-2.0) \\
4.0(2.0-6.0)\end{array}$ \\
\hline
\end{tabular}




\subsubsection{USPIO ENHANCEMENT}

At baseline, there were no differences in R2* values between healthy volunteers and patients with myocarditis (pan-myocardium and LGE+ regions; Figures 5.1 and 5.2). After USPIO administration, the R2* in all three groups increased but there was no difference in either the change in, or post USPIO, R2* between the groups (Figure 5.2 and Table 5.2).

\subsubsection{MYOCARDIAL OEDEMA}

There was no difference in pan-myocardial T2 between volunteers and patients with myocarditis. Regions displaying LGE in myocarditis patients had higher T2 than pan-myocardial values for both healthy volunteers and patients with myocarditis $(p<0.0001$ and $p<0.01$ respectively; Figure 5.2 and Table 5.2).

\subsubsection{BASELINE VERSUS 3-MONTH IMAGING IN MYOCARDITIS PATIENTS}

There were no changes in myocardial USPIO uptake between baseline and 3 months either on pan-myocardial analysis (mean $+7.6 \mathrm{~s}^{-1} ; 95 \% \mathrm{CI}-4.8$ to 19.9 ) or in regions with LGE (mean $-4.2 \mathrm{~s}^{-1}$; 95\% CI -21.9 to 13.5 ; $\mathrm{p}>0.05$ for both; Figure 5.3). Panmyocardial T2 did not change over 3 months (mean -1.1 ms; 95\% CI -5.8 to 3.5; $\mathrm{p}>0.05$ ). Volume of LGE and T2 within regions displaying LGE reduced over the 3-month period (mean $-19.7 \mathrm{~mL}$; $95 \%$ CI -0.5 to -40.0 and mean $-5.8 \mathrm{~ms}$; $95 \% \mathrm{CI}$ -0.9 to -10.7 respectively; $\mathrm{p}<0.05$ for both). Ejection fraction increased over the 3-month period (mean $+6 \%$; $95 \%$ CI 0.5 to 11.5 ; $\mathrm{p}<0.05$ ). 

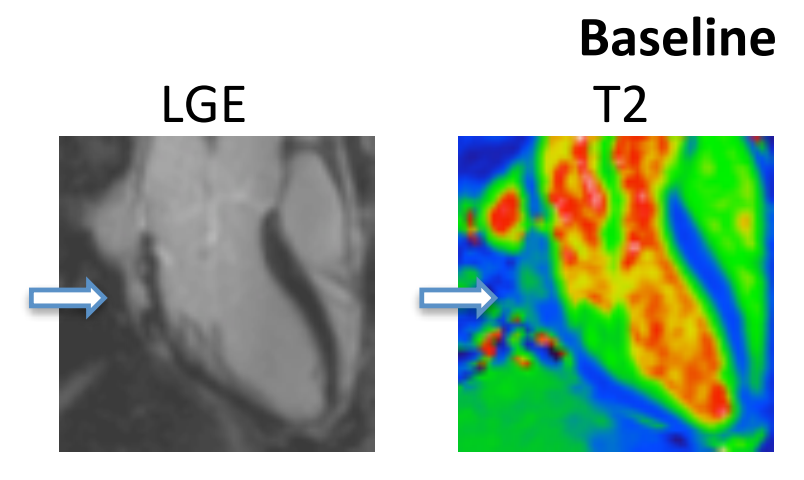

\section{4h Post USPIO R2*}
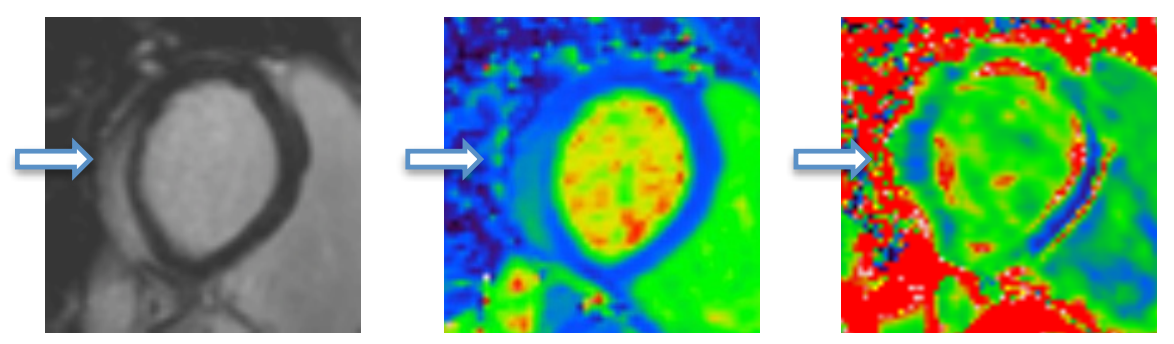

\section{3months}
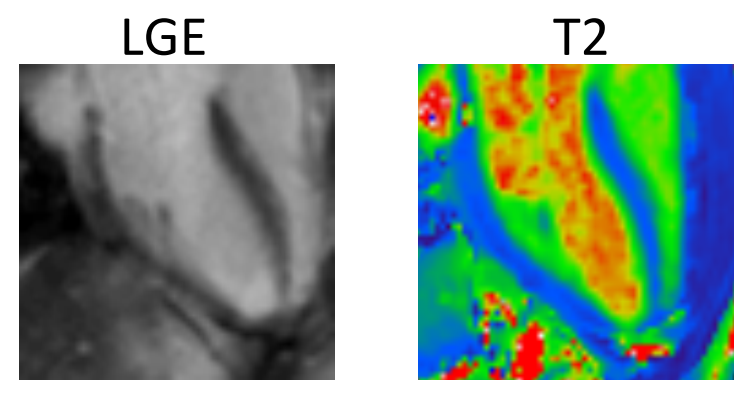

24h Post USPIO R2*
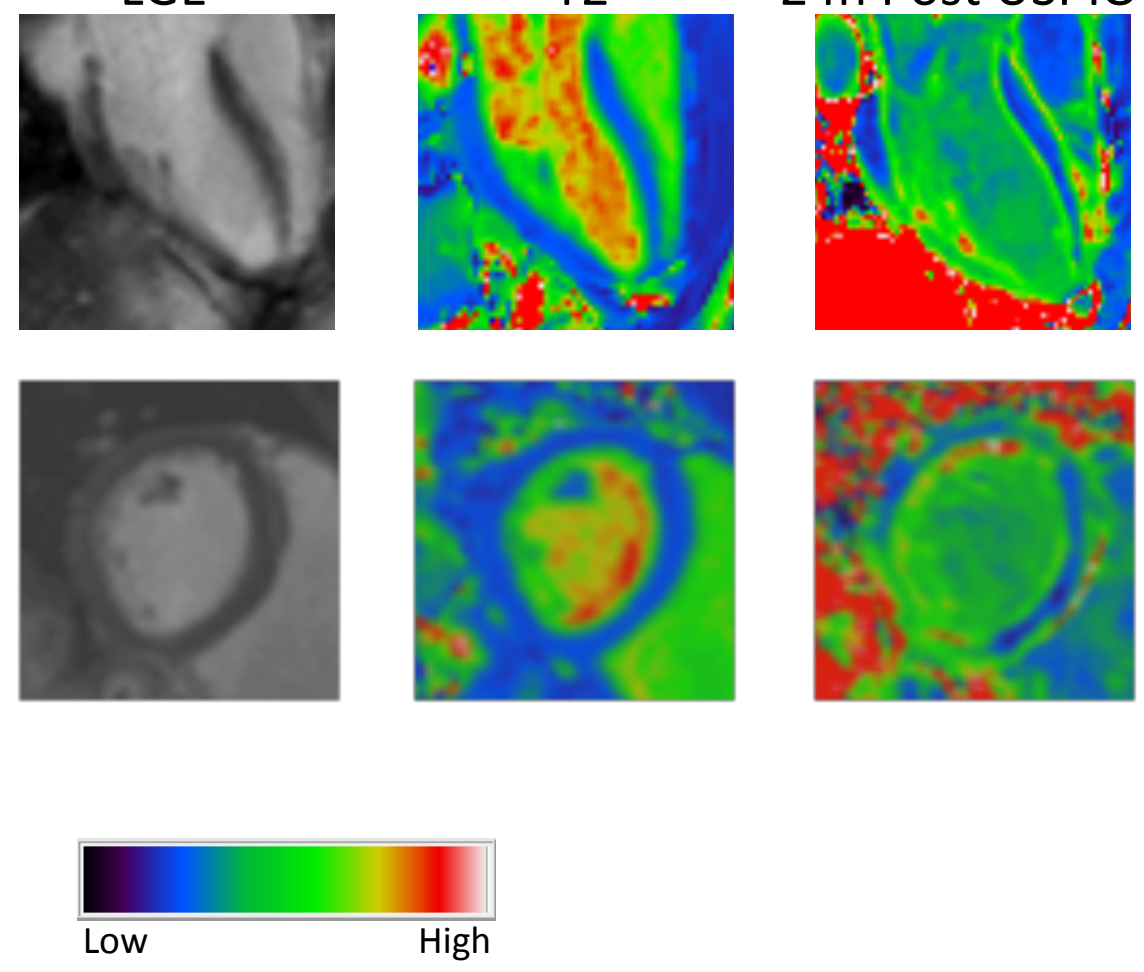

Figure 5.1. Images of a patient with myocarditis. Three chamber and basal short axis images of a patient with myocarditis displaying patchy posterolateral LGE (white regions, arrowed) that correspond to oedematous regions of myocardium on the T2 map (lighter regions, arrowed). There is no uptake of USPIO in the post USPIO R2* maps; corresponding regions may even have lower R2* in this patient. Myocardial oedema appears to normalise but subtle LGE remains at 3 months. LGE - late gadolinium enhancement; h - hours; USPIO - ultrasmall superparamagnetic particles of iron oxide. 
Myocardial USPIO enhancement

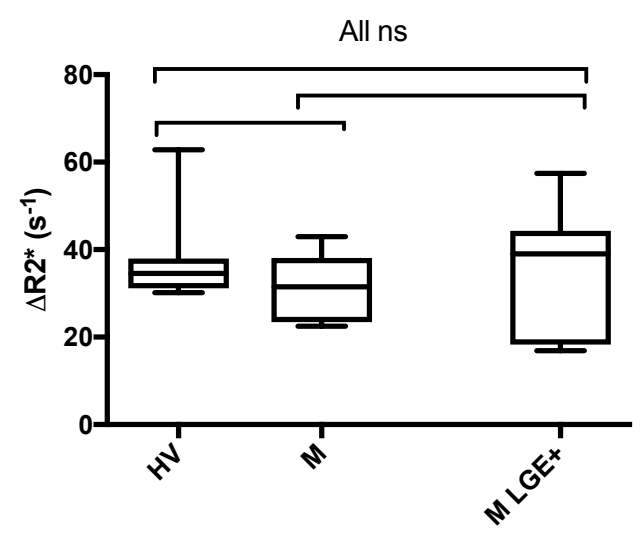

Myocardial Oedema

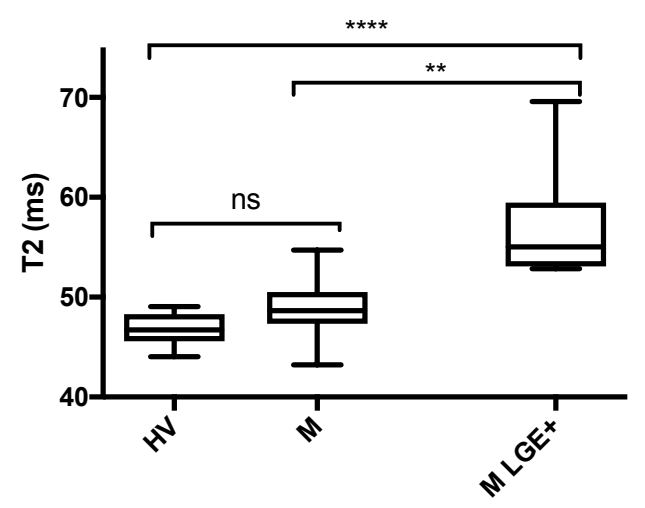

Figure 5.2. Myocardial USPIO enhancement versus oedema at baseline. Changes in myocardial R2* due to USPIO accumulation (A, left) and myocardial oedema by T2 mapping $(\mathbf{B}$, right) are shown in healthy volunteers (HV) and patients with myocarditis (M) using pan-myocardial average, and further limited to regions displaying LGE in patients with myocarditis (M LGE+). There were no significant differences in $\triangle \mathrm{R} 2 *$ due to USPIO accumulation between all groups ( $\mathrm{p}>0.05$ for all). There was no difference in pan-myocardial T2 between volunteers and patients with myocarditis. Myocardial regions displaying LGE $(\mathrm{M} \mathrm{LGE}+)$ had greater $\mathrm{T} 2$ than the pan-myocardial value for healthy volunteers and myocarditis patients $(* * * *=p<0.0001$ and $* *=p<0.01$ respectively). USPIO ultrasmall superparamagnetic particles of iron oxide; ns - not significant; LGE - late gadolinium enhancement. 
TABLE 5.2 Myocardial R2* and T2 values in healthy volunteers and patients with myocarditis

\begin{tabular}{lccc}
\hline & $\begin{array}{c}\text { Healthy Volunteers } \\
\text { (Pan-myocardial) }\end{array}$ & $\begin{array}{c}\text { Myocarditis } \\
\text { (Pan-myocardial) }\end{array}$ & $\begin{array}{c}\text { Myocarditis } \\
\text { (LGE+ regions) }\end{array}$ \\
\hline $\begin{array}{l}\text { Pan-myocardial pre } \\
\text { USPIO R2* }\left(\mathrm{s}^{-1}\right)\end{array}$ & $46.9 \pm 4.1$ & $43.7 \pm 5.3$ & $51.8 \pm 8.3$ \\
$\begin{array}{l}\text { Pan-myocardial post } \\
\text { USPIO R2* }\left(\mathrm{s}^{-1}\right)\end{array}$ & $84.2 \pm 12.4$ & $75.6 \pm 11.3$ & $86.1 \pm 15.3$ \\
$\begin{array}{l}\text { Pan-myocardial } \\
\mathbf{A R 2} * \mathrm{~s}^{-1} \text { ) }\end{array}$ & $37.2 \pm 9.6$ & $31.4 \pm 7.5$ & $35.0 \pm 15.0$ \\
$\begin{array}{l}\text { T2 (ms) } \\
\text { mean } \pm \text { SD. }\end{array}$ & $46.7 \pm 1.6$ & $48.9 \pm 3.1$ & $57.1 \pm 5.3 * * * *$ \\
$\begin{array}{l}* * * * \\
\text { superparamagnetic particles of iron oxide. }\end{array}$ & & & \\
\end{tabular}




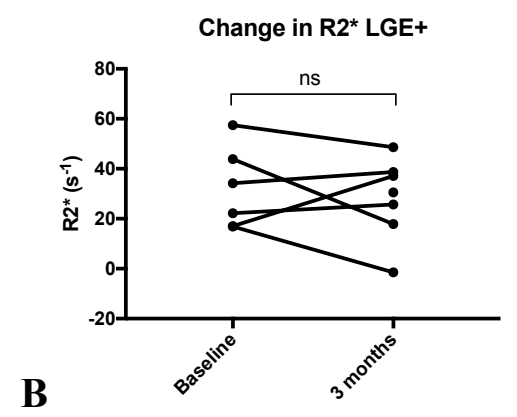

A

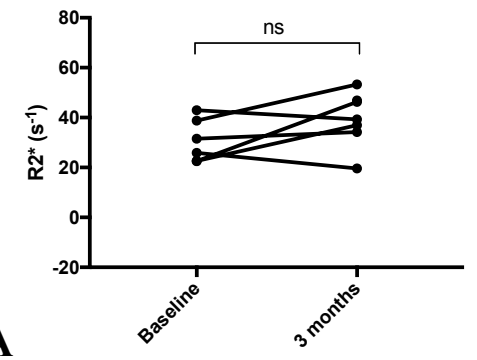

Change in T2 - pan-myocardium

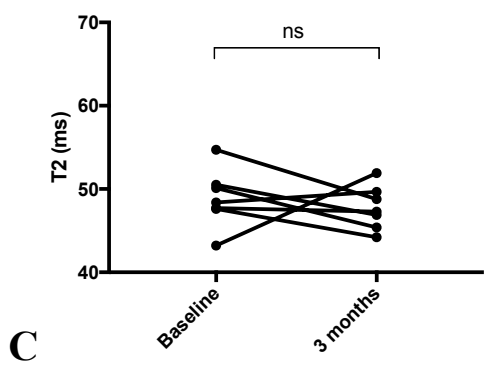

$\mathbf{E}$

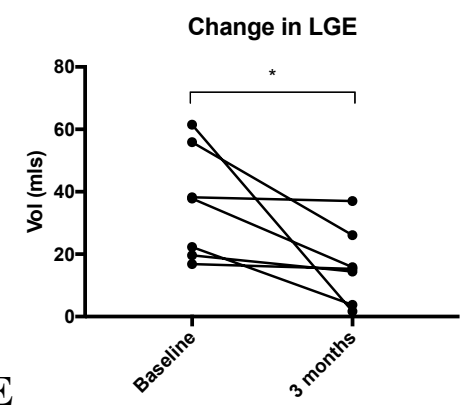

B

Change in T2 - LGE+

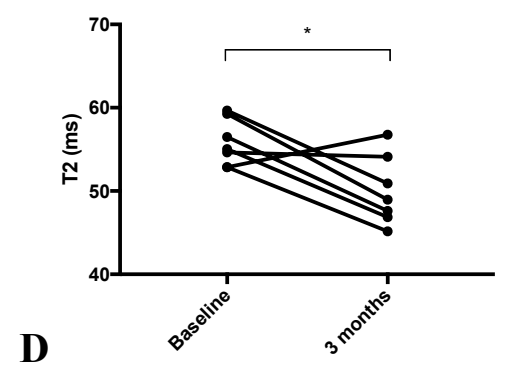

$\mathbf{F}$

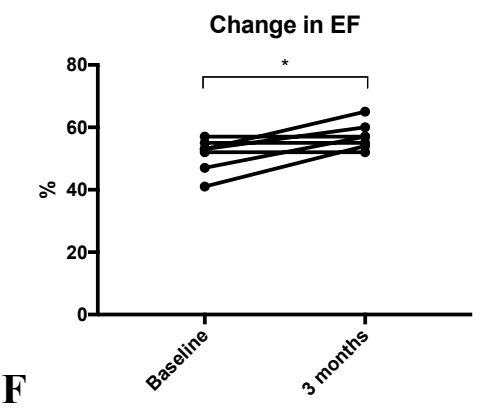

Figure 5.3. Changes on repeat imaging at 3 months in patients with myocarditis. There was no significant (ns) difference in USPIO uptake between baseline and 3 months in both pan-myocardium (A) and in regions displaying LGE (B). There was no difference in pan-myocardial T2 (C) over 3 months. T2 in regions displaying LGE (D) reduced, as did the volume of LGE (E) over the 3-month period There was an increase in ejection fraction (EF) between baseline and 3 months (F) $(*=\mathrm{p}<0.05)$. USPIO - ultrasmall superparamagnetic particles of iron oxide; LGE - late gadolinium enhancement. 


\subsection{DISCUSSION}

For the first time, I report the combined assessment of myocarditis using late gadolinium enhancement, T2 mapping and USPIO-enhanced T2* MRI. USPIOenhanced T2* MRI has previously been used in man to assess cardiovascular inflammation in a range of different conditions [Tang et al 2009; Richards et al 2011; Alam et al 2012; Yilmaz et al 2013], with preliminary positive results from a rodent model of myocarditis. This raised hope that USPIO-enhanced T2*MRI might add useful cell-specific clinical information in patients with myocarditis. However, whilst I found typical features of myocarditis using standard imaging with LGE and T2 mapping CMR, I did not detect USPIO enhancement within the myocardium of our patients. This suggests that USPIO-enhanced MRI provides no additional diagnostic value in these patients and that tissue-resident macrophages are not a major contributor to the cellular inflammation following acute myocarditis.

My case study population of 9 patients were excellent examples of myocarditis. They were young, with significantly elevated levels of systemic inflammatory markers, including neutrophil count, CRP and cardiac specific high-sensitivity troponin, and all had typical features of myocarditis on CMR reported by two independent clinical radiologists. Most patients had ECG changes suggesting significant myocyte injury and several had normal coronary angiograms. All patients that did not have a coronary angiogram did not have any risk factors for coronary artery disease. I detected MRI features of myocarditis in every patient. Late gadolinium enhancement was discontinuous in nature and generally epicardial and subepicardial in distribution, but could be found in the midwall and occasionally in the subendocardium. LGE was 
never found in the subendocardium alone or confined to one coronary territory that would be more suggestive of myocardial infarction. In keeping with the typical distribution of myocarditis, most patients displayed LGE in the inferior, posterior and lateral walls. We are therefore confident that the diagnosis of myocarditis was robust in all patients, and an independent cardiologist verified this.

Myocardial oedema was easily visualised, and indeed quantified, using T2 mapping (Figure 5.2). I found intense myocardial oedema in our myocarditis cohort with maximal $\mathrm{T} 2$ values approaching $70 \mathrm{~ms}$ on $\mathrm{T} 2$ mapping. Within regions displaying LGE (LGE+), there was a profound increase in T2 when compared to volunteers. This signal was so powerful that a trend remained evident when comparing pan-myocardial T2 values to those of volunteers, although the difference was not statistically significant. Seven patients returned for repeat imaging assessment at 3 months, where we recorded an improvement in clinical features: a reduction in the volume of LGE (by around 50\%); a reduction in oedema within inflamed (LGE+) regions, and an overall improvement in ejection fraction.

I was unable to detect USPIO accumulation in our cohort of patients with myocarditis. I know that macrophages can be present in myocarditis, although they are generally not the predominant cell type. It is possible that patients within my group had predominantly lymphocytic or neutrophil rich cell infiltrate, and the finding of greater levels of neutrophils in patients with myocarditis at baseline may support the latter. The clinical care team did not feel that endomyocardial biopsy was justified in any of the cases, and lack of histological data is clearly a limitation. In 
recent work, I have detected USPIO-laden macrophages in patients with recent myocardial infarction and demonstrated macrophage uptake of USPIO in biopsies taken at the time of cardiac surgery [Stirrat et al 2017]. I therefore acknowledge that while some USPIO-laden macrophages may have been present in patients with myocarditis, there were either insufficient numbers of macrophages or USPIO engulfment was inhibited or deficient. Ultimately USPIO-enhanced T2* CMR was unable to demonstrate increased macrophage activity and lacked sensitivity for detecting myocarditis.

A further contributory reason for failure to detect USPIO enhancement within inflamed regions may be due to difficulties encountered in USPIO-enhanced T2* imaging. These are discussed in greater detail elsewhere [Stirrat et al 2016] but artefact due to breathing and in particular, 'blooming' artefacts from the vascular organs of the lungs, liver and stomach can be problematic. This can make analysis more challenging by having to exclude the affected later echo times from $\mathrm{T} 2 *$ decay curve fitting. Indeed, blooming artefacts from nearby tissues and organs most commonly affect the inferior and lateral walls, which is the usual site of inflammation in myocarditis. Therefore the myocardial regions of greatest interest were often the most difficult to analyse although this can usually be overcome [Stirrat et al 2016]. This may go some way to explain why there is greater spread of R2* data in patients with myocarditis, especially in LGE+ regions. Finally, patients with myocarditis often had symptoms of pleurisy and pericarditis, and despite adequate analgesia, often found the long breath-holding required for $\mathrm{T} 2 *$ imaging difficult. 


\subsubsection{LiMitATIONS}

Some final limitations also deserve mention. First, the sample size was small, but numbers in this pilot study were sufficient to show that USPIO-enhanced T2* CMR fails to add clinically relevant information to CMR imaging parameters of LGE and T2 mapping for individual patients. Second, the control volunteer group was not age or sex matched but we have no reason to believe that this influenced the results. Lastly, myocardial geometry is affected by several factors that vary between scans such as presence of tissue oedema, heart rate and blood volume status, and accurate co-registration can be challenging.

Despite these results, USPIO-enhanced imaging still holds promise as a non-invasive imaging method for the diagnosis and monitoring of tissue inflammatory macrophages in the heart. We have recently found that USPIO-enhanced MRI can detect and serially monitor macrophages after myocardial infarction [Stirrat et al 2017] and work is underway at our centre as part of this project (NCT02319278) to assess the value of this technique in diagnosing cardiac sarcoidosis and cardiac transplant rejection. If successful, USPIO-enhanced MRI may provide a platform on which to assess existing and novel therapeutic interventions that might modify the inflammatory process, not only after MI but also in other inflammatory processes affecting the heart. 


\subsubsection{Conclusion}

In conclusion, I have shown that in patients with acute myocarditis, USPIOenhanced T2* MRI does not provide additional clinically relevant information to that of LGE and T2 mapping MRI. This suggests that tissue-resident macrophages do not provide a substantial contribution to the myocardial inflammation in this condition. 
CHAPTER 6

FERUMOXYTOL-ENHANCED MAGNETIC

RESONANCE IMAGING IN PATIENTS WITH PRIOR

CARDIAC TRANSPLANTATION 


\subsection{SUMMARY}

Background Ultrasmall superparamagnetic particles of iron oxide-enhanced magnetic resonance imaging can detect cellular inflammation within tissues and may help non-invasively identify cardiac transplant rejection. Here, I aimed to determine the normal reference values for USPIO-enhanced MRI in patients with a prior cardiac transplant, and examine whether USPIO-enhanced MRI could detect myocardial inflammation in patients with transplant rejection.

Methods Ten volunteers and 11 patients with cardiac transplant underwent T2, T2* and late gadolinium enhancement 1.5 T MRI, with further T2* imaging at 24 hours after USPIO (ferumoxytol, $4 \mathrm{mg} / \mathrm{kg}$ ) infusion, at baseline and 3 months.

Results Ten patients with clinically stable cardiac transplantation and nine healthy volunteers were retained for analysis. Myocardial T2 values were higher in patients with cardiac transplant versus healthy volunteers $(53.8 \pm 5.2$ versus $48.6 \pm 1.9 \mathrm{~ms}$ respectively; $\mathrm{p}=0.003$ ). There were no differences in the magnitude of USPIOinduced change in $\mathrm{R} 2 *$ in patients with transplantation (change in R2*, $26.6 \pm 7.3$ versus $22.0 \pm 10.4 \mathrm{~s}^{-1}$ in healthy volunteers; $\mathrm{p}=0.28$ ). After 3 months, patients with transplantation $(\mathrm{n}=5)$ had unaltered T2 values $(52.7 \pm 2.8$ versus $52.12 \pm 3.4 \mathrm{~ms} ; \mathrm{p}=0.80)$ and changes in $\mathrm{R} 2 *$ following USPIO (29.42 \pm 8.14 versus $\left.25.8 \pm 7.8 \mathrm{~s}^{-1} ; \mathrm{p}=0.43\right)$.

Conclusion Stable patients with cardiac transplantation have increased myocardial T2 values, consistent with resting myocardial oedema or fibrosis. In contrast, USPIO-enhanced MRI is normal and stable over time suggesting the absence of chronic macrophage-driven cellular inflammation. It remains to be determined 
whether USPIO-enhanced MRI may be able to identify acute cardiac transplant rejection.

\subsection{INTRODUCTION}

Cardiac transplantation is a life prolonging treatment for end stage cardiac disease. Transplant rejection is a major threat to the allograft, occurring in $20-30 \%$ of recipients in the first year [Hertz et al 2009], but can occur at any stage after transplantation and causes significant morbidity and mortality. Rejection is notoriously difficult to diagnose using existing non-invasive imaging methods necessitating repeated surveillance myocardial biopsies.

Most cases of acute rejection are due to cellular rejection with antibody-mediated rejection less prevalent. Rejection severity is classified according to histological findings, and although the cellular infiltrate in acute cellular rejection is predominantly lymphocytic, macrophage infiltration has a key role [Stewart et al 2005; Mannon 2012]. The importance of macrophages in acute cardiac allograft rejection was recently emphasised in a rodent study that showed depletion of circulating macrophages protected the allograft against rejection, raising the possibility of therapeutic targeting of macrophages as a novel treatment strategy [Wu et al 2013].

Iron oxide nanoparticles are generating interest as an MR imaging contrast medium that is able to detect macrophages, and clinical applications, such as myocardial 
infarction, are now emerging [Trivedi 2004; Tang et al 2006; Trivedi 2006; Tang et al 2009; Richards et al 2011; Alam et al 2012; Yilmaz et al 2013; McBride et al 2015; Stirrat et al 2017]. Ultrasmall superparamagnetic particles of iron oxide consist of an iron oxide core surrounded by a carbohydrate or polymer coating. They are small enough to extravasate passively through capillaries, where they are engulfed by tissue-resident macrophages [Ruehm et al 2001], and are detectable by T2*-weighted MRI. Thus USPIO-enhanced MRI can identify tissue-resident macrophage activity and help to identify cellular inflammation within tissues.

Promising preclinical studies have shown USPIO-enhanced MRI is able to detect acute cardiac and renal allograft rejection with USPIO signal correlating with macrophage distribution, rejection severity on histology, and impaired cardiac function. Moreover, this approach can also be used to assess treatment response with rodent models demonstrating less USPIO enhancement following initiation of immunosuppression [Kanno et al 2001; Johansson et al 2002; Penno et al 2005; Wu et al 2009; Wu et al 2013; Alam et al 2015]. A future role of USPIO includes a 'theranostic' strategy whereby imaging is combined with therapy. For example, Guo et al recently conjugated an iron nanoparticle to a $\mathrm{CD} 3$ antibody and a therapy gene, allowing imaging and targeting of $\mathrm{T}$ cells that play a central role in acute cardiac allograft rejection [Guo et al 2012].

In this study, I aimed to assess and quantify myocardial USPIO enhancement in stable patients with cardiac transplantation and patients with cardiac transplant rejection, correlating enhancement with clinical measures of inflammation and 
oedema including T2 mapping MRI; a quantitative imaging method assessing myocardial oedema in transplant rejection [Giri et al 2009; Thavendiranathan et al 2012]. I hypothesised that USPIO-enhanced MRI would detect myocardial macrophage activity in the inflamed myocardium of rejecting transplanted hearts, but not in stable healthy cardiac allografts, and provide a cellular-specific non-invasive imaging technique that may aid and improve patient diagnosis and management.

\subsection{METHODS}

This was an open label observational multicentre cohort study (NCT02319278). The study was performed in accordance with the Declaration of Helsinki, the approval of the Scotland A Research Ethics Committee (13-SS-0111), and the written informed consent of all participants. The Medicines and Healthcare products Regulatory Agency of the United Kingdom gave Clinical Trial Authorisation for the study (EUDraCT 2013-002336-24).

\subsubsection{STUDY POPULATIONS}

Adult ( $>18$ years of age) patients with a history of cardiac transplantation (including suspected allograft rejection) were recruited into the study. Healthy volunteers had no clinically significant past medical history. Exclusion criteria were contraindication to MR imaging or ferumoxytol infusion, any other inflammatory comorbidity, renal failure (estimated glomerular filtration rate $<30 \mathrm{~mL} / \mathrm{min} / 1.73 \mathrm{~m}^{2}$ ), pregnancy, breastfeeding and women of childbearing potential without reliable contraception. 


\subsubsection{STUDY PROTOCOL}

Patients with cardiac transplantation and healthy volunteers underwent paired MRI scans at baseline, and patients were invited to return for repeat imaging after 3 months. At the time of scanning, blood samples were collected for clinical haematology and biochemistry measurements. The troponin assay used was the Elecsys ${ }^{\circledR}$ high sensitivity Troponin T assay (Roche Diagnostics).

\subsubsection{MAGNETIC RESONANCE IMAGING}

MRI was performed using a MAGNETOM Avanto $1.5 \mathrm{~T}$ MRI scanner, with a dedicated cardiac array coil. All images were acquired with electrocardiogram gating using expiration breath-holds. Routine steady state free precession sequences were used to acquire long and short axis cine images of the heart (TR $85.8 \mathrm{~ms}$, TE $1.45 \mathrm{~ms}$, flip angle $50^{\circ}$, matrix $173 \times 256$, FoV $400 \mathrm{~mm}$, slice thickness $8 \mathrm{~mm}, 2 \mathrm{~mm}$ gap). Quantitative USPIO imaging was performed in similar slice positions using a prototype $\mathrm{T} 2 *$-weighted multi-gradient-echo acquisition with a volumetric shim applied over the entire heart volume (TR 996 ms, TE 2.13, 4.3, 6.4, 8.6, 10.7, 12.8, 15.0, $17.1 \mathrm{~ms}$, flip angle $18^{\circ}$, matrix 130x256, FoV $400 \mathrm{~mm}$, slice thickness $6 \mathrm{~mm}$, gap $4 \mathrm{~mm}$ ). The T2*-weighted acquisitions included views through the liver, spleen and spine to allow quantification of USPIO accumulation within organs of the reticuloendothelial system. The same $\mathrm{T}^{*}$ protocol was used to quantify USPIO accumulation 24 hours after infusion allowing calculation of $\mathrm{T} 2 *$ relaxation rates before and after administration of USPIO. T2 mapping was conducted using a Siemens prototype T2-prepared TrueFISP acquisition acquiring identical long and short axis slice positions (TR $219.3 \mathrm{~ms}$, TE $1.07 \mathrm{~ms}$, T2P durations $0,25,50 \mathrm{~ms}$, 
flip angle $70^{\circ}$, matrix 130x192, FoV $400 \mathrm{~mm}$, slice thickness $6 \mathrm{~mm}, 4 \mathrm{~mm}$ gap). T2P-TrueFisp images are acquired at intervals of at least $3 \mathrm{RR}$ intervals to allow for sufficient magnetisation recovery in between acquisitions.

Immediately after the baseline $\mathrm{T} 2$ and $\mathrm{T} 2 *$-weighted scan, participants received an intravenous administration of gadolinium contrast medium $(0.15 \mathrm{mmol} / \mathrm{kg})$ followed by breath held inversion recovery sequences in long axis and short axis planes to acquire late enhancement images. Optimal inversion time was determined on a sliceby-slice basis using standard late enhancement TI-scout protocols (TR $750 \mathrm{~ms}$, TE $2.61 \mathrm{~ms}$, flip angle $20^{\circ}$, matrix $173 \times 256$, FoV $400 \mathrm{~mm}$, slice thickness $9 \mathrm{~mm}$, gap $1 \mathrm{~mm})$. The inversion recovery late enhancement short axis slices were acquired using similar slice positions as the $\mathrm{T} 2$ oedema and $\mathrm{T} 2 *$-weighted imaging.

\subsubsection{USPIO}

Intravenous infusion of USPIO (ferumoxytol, $4 \mathrm{mg} / \mathrm{kg}$; Rienso®) was performed immediately following the baseline magnetic resonance scan over 15 minutes using a concentration of $2-8 \mathrm{mg} / \mathrm{mL}$, diluted in $0.9 \%$ saline or $5 \%$ dextrose. Haemodynamic monitoring was conducted throughout and participants were observed for a further 30 minutes to ensure no hypersensitivity reactions.

\subsubsection{IMAGE ANALYSIS}

All T2*-weighted multi-gradient-echo images for each patient were analysed using Circle CVI software. An experimentally determined threshold used in previous work [Alam et al 2012] for the coefficient of determination $\left(\mathrm{r}^{2}>0.85\right)$ was used to exclude 
data that did not have an acceptable exponential decay when signal intensity was plotted against echo time. Individual images affected by artefact were excluded. The inverse of the mean $\mathrm{T} 2 *(\mathrm{R} 2 *)$ for each ROI was then calculated to assess the uptake of USPIO, where the higher the value, the greater the USPIO accumulation.

T2 maps, ventricular volume and functional analyses were also performed using Circle CVI software. T2 and T2* data were collected immediately prior to USPIO administration. USPIO-enhanced $\mathrm{T} 2 *$ data were collected $24-25$ hours following ferumoxytol administration. Regions of interest were drawn on $\mathrm{T} 2$ and $\mathrm{T} 2 *$ images in the septum at mid cavity level between anterior and inferior right ventricular (RV) insertion points (Figure 6.1). Septal myocardial regions only were selected to reduce influence of artefact caused by nearby stomach or lung [Stirrat et al 2016]. 
$\mathrm{R} 2 * \operatorname{map}$

\section{A - Healthy Volunteer}

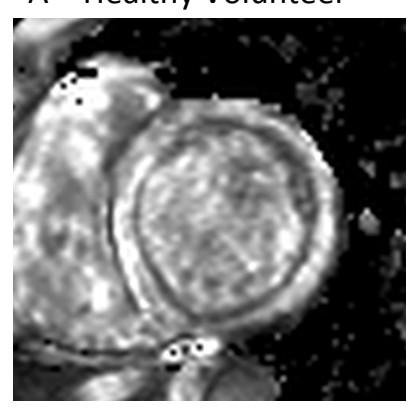

\section{B - Cardiac Transplant}

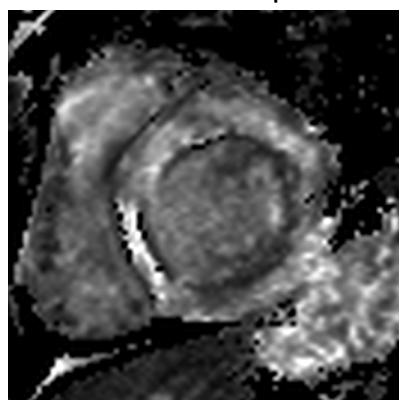

\section{C - Myocardial Infarction}

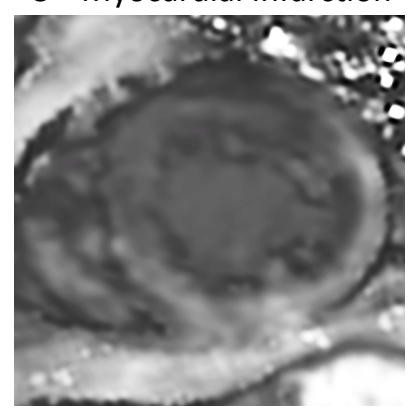

T2 Map
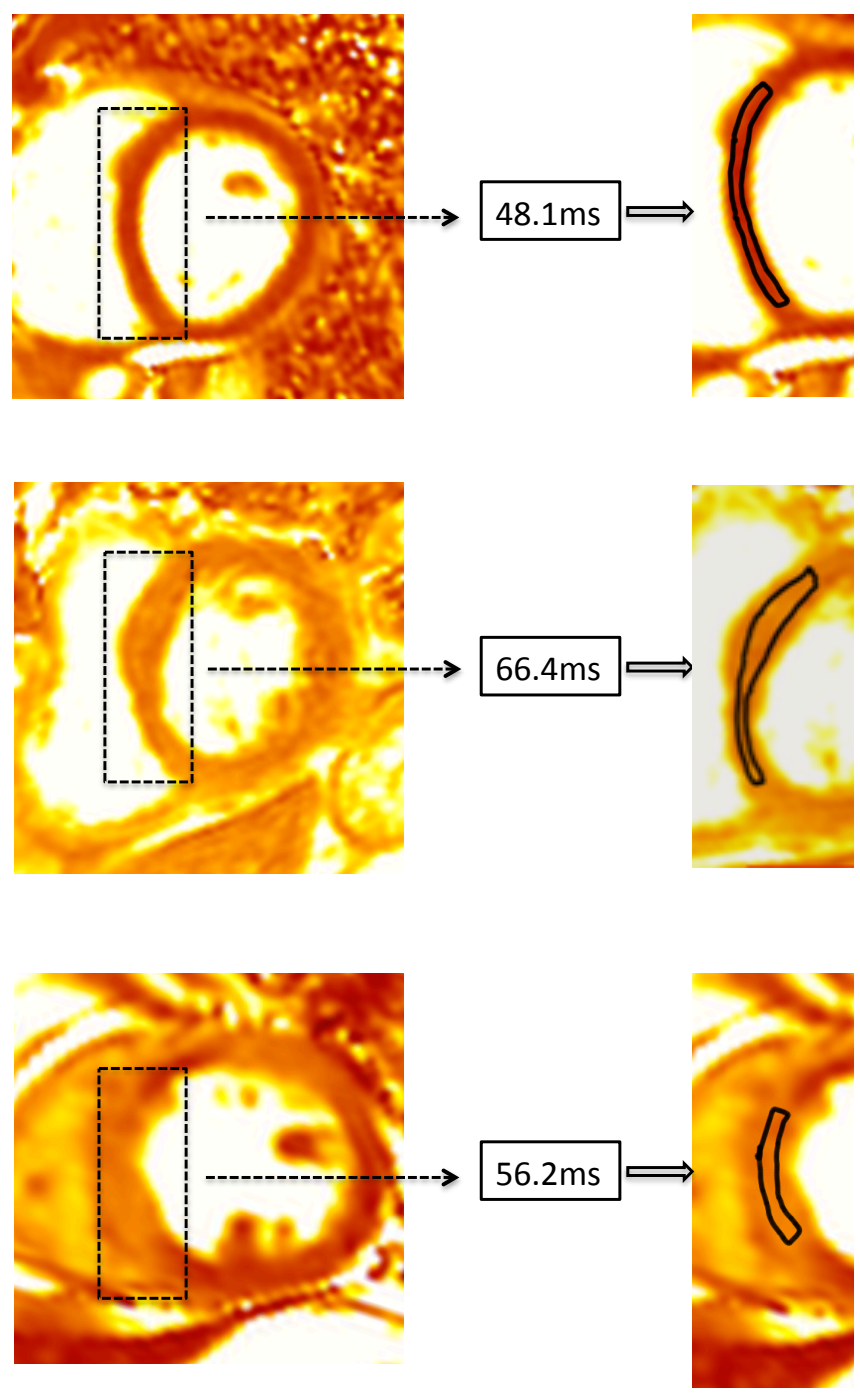

Figure 6.1. Post USPIO R2* and T2 maps for a healthy volunteer (A), patient with cardiac transplant (B) and myocardial infarction (C). Myocardial oedema is displayed as brighter signal in the T2 colour map corresponding to higher T2 value. Cardiac transplant myocardium (B) displays no USPIO accumulation but high T2, in contrast to USPIO accumulation and high $\mathrm{T} 2$ in myocardial infarction (C). USPIO - ultrasmall superparamagnetic particles of iron oxide. 


\subsubsection{Histology}

Myocardial tissue samples were obtained from one patient with cardiac transplantation undergoing surveillance biopsies without suspicion of transplant rejection. USPIO was administered 24 hours prior to the biopsy, and a trucut myocardial biopsy sample was taken from the myocardium. The biopsy sample was fixed in formalin, embedded in paraffin, sectioned, and stained to look at architecture (haematoxylin and eosin), accumulation and distribution of USPIO (Prussian Blue) and macrophages (CD68).

\subsubsection{STATISTICAL ANALYSIS}

All statistical analysis was performed with GraphPad Prism, version 6. Shapiro-Wilk or D'Agostino and Pearson tests were used to test normality of distribution. To compare participant characteristics, USPIO uptake and myocardial oedema in patients and volunteers, R2* and T2 values were compared using Chi-squared, unpaired $t$-tests, and Mann-Whitney tests depending on normality of data. To compare results at 3 months with baseline, paired $t$-test and Wilcoxon test was used depending on normality of distribution. Statistical significance was defined as twosided $\mathrm{p}<0.05$. 


\subsection{RESULTS}

Ten volunteers and 11 patients with cardiac transplantation were recruited. One volunteer was excluded from analysis due to the presence of late gadolinium enhancement as described previously [Stirrat et al 2016]. At the time of recruitment, all transplant patients were assessed to be clinically well with no firm evidence of allograft rejection. One patient with prior cardiac transplant was excluded due the finding of LGE consistent with myocardial infarction that may influence the USPIO enhancement data [Alam et al 2012; Stirrat et al 2017]. No other patient with cardiac transplant displayed LGE. Five of the 10 cardiac transplant patients returned at 3 months for repeat imaging. Administration of ferumoxytol was well tolerated with no adverse reactions reported during or immediately after administration in any of the participants.

Volunteers were predominantly female and patients with cardiac transplant were predominantly male (Table 6.1). Transplant patients had smaller indexed enddiastolic ventricular volumes $(\mathrm{p}<0.01)$ and higher baseline plasma high-sensitivity troponin concentrations $(\mathrm{p}<0.05)$. There were more males in the transplant group $(p<0.05)$ but there were no other differences between groups at baseline.

\subsubsection{T2 AND R2* MAPPING}

Patients with cardiac transplantation had a higher T2 value than volunteers $(53.8 \pm 5.2$ versus $48.6 \pm 1.9 \mathrm{~ms}$ respectively; $\mathrm{p}=0.003$; Figures 6.1 and 6.2 , and Table 6.2 ). 
TABLE 6.1 Participant Characteristics

\begin{tabular}{lll}
\hline & Healthy Volunteers & $\begin{array}{l}\text { Patients with Cardiac } \\
\text { Transplant }\end{array}$ \\
\hline Number & 9 & 10 \\
Female & 6 & $1 *$ \\
Age (years) & $52(45.5-61.5)$ & $60(52.75-64.5)$ \\
Time since transplantation (months) & & $59(19-159)$ \\
Body mass index $\left(\mathrm{kg} / \mathrm{m}^{2}\right)$ & $22.9(20.1-26.9)$ & $25.9(24.0-27.9)$ \\
Left ventricular end-diastolic & $80.9 \pm 10.4$ & $62.9 \pm 15.5^{* *}$ \\
volume $\left(\mathrm{mL} / \mathrm{m}^{2}\right)$ & & \\
Left ventricular ejection & $63.6 \pm 4.9$ & $65.0 \pm 7.1$ \\
fraction $(\%)$ & &
\end{tabular}

Blood Tests

$\begin{array}{lll}\text { White cell count }\left(\mathrm{x} 10^{9} / \mathrm{L}\right) & 6.5 \pm 1.5 & 6.1 \pm 2.5 \\ \text { C-reactive protein }(\mathrm{mg} / \mathrm{L}) & 1.9 \pm 2.0 & 2.1 \pm 2.1 \\ \text { Plasma troponin }(\mathrm{ng} / \mathrm{L}) & 3.1 \pm 2.8 & 14.1 \pm 20.9^{*}\end{array}$

Mean \pm SD or median (interquartile range).

${ }^{*} \mathrm{p}<0.05,{ }^{* *} \mathrm{p}<0.01$ (compared to volunteers). SD - standard deviation. 


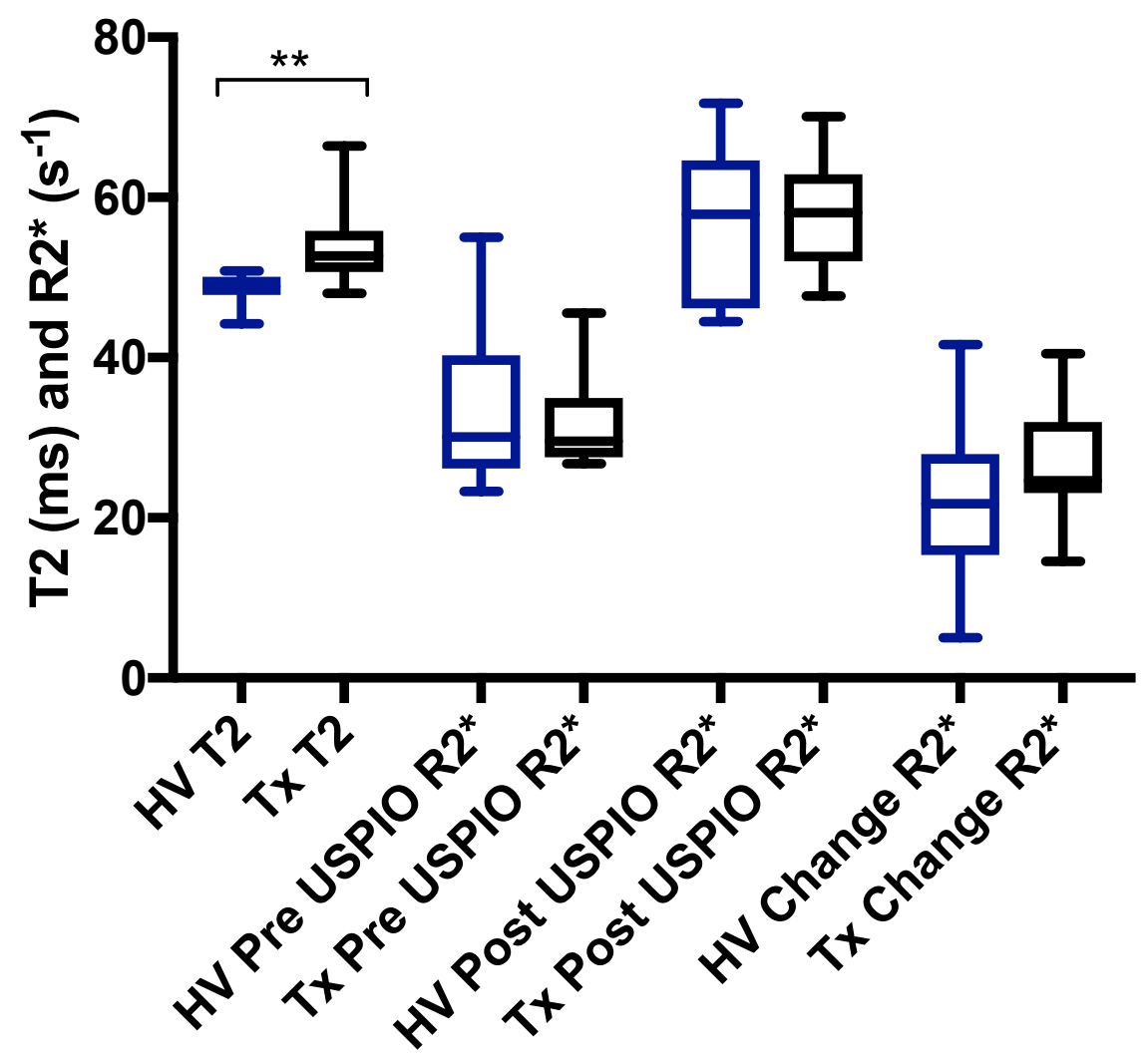

Figure 6.2. Comparison of $\mathrm{T} 2(\mathrm{~ms})$ and $\mathrm{R} 2 *\left(\mathrm{~s}^{-1}\right)$ measurements at baseline in healthy volunteers (HV, blue) and cardiac transplant patients (Tx, black). Transplant patients have higher T2 than healthy volunteers, but no other significant differences exist between groups $(* *=p<0.01)$. USPIO ultrasmall superparamagnetic particles of iron oxide. 
TABLE 6.2 Septal myocardial $\mathrm{R}^{*}$ * and $\mathrm{T} 2$ values in healthy volunteers and patients with cardiac transplantation

\begin{tabular}{lll}
\hline & $\begin{array}{c}\text { Patients with } \\
\text { Cardiac Transplant }\end{array}$ & Healthy Volunteers \\
\hline Pre USPIO R2* $\left(\mathrm{s}^{-1}\right)$ & $31.6 \pm 5.9$ & $34.0 \pm 10.1$ \\
Post USPIO R2* $\left(\mathrm{s}^{-1}\right)$ & $58.2 \pm 7.5$ & $56.0 \pm 10.2$ \\
$\mathbf{\Delta R 2} *\left(\mathrm{~s}^{-1}\right)$ & $26.6 \pm 7.3$ & $22.0 \pm 10.4$ \\
$\mathbf{T} 2(\mathrm{~ms})$ & $53.8 \pm 5.2^{* *}$ & $48.6 \pm 1.9$ \\
$\begin{array}{l}\text { Mean } \pm \mathrm{SD} \text {. } \\
\text { p }<0.01 \text { (compared to volunteers). USPIO - ultrasmall superparamagnetic particles of iron oxide; SD - standard } \\
\text { deviation. }\end{array}$ &
\end{tabular}


There were no differences in R2* between patients with cardiac transplantation and volunteers at baseline (31.6 \pm 5.9 versus $\left.34.0 \pm 10.1 \mathrm{~s}^{-1} ; \mathrm{p}=0.84\right)$, at 24 hours after USPIO administration (58.2 \pm 7.5 versus $\left.56.0 \pm 10.2 \mathrm{~s}^{-1} ; \mathrm{p}=0.60\right)$, or in the magnitude of change in R2* due to USPIO (26.6 \pm 7.3 versus $22.0 \pm 10.4 \mathrm{~s}^{-1}$; $\mathrm{p}=0.28$; Figures 6.1 and 6.2, and Table 6.2).

There were no differences in either T2 $(52.7 \pm 2.8$ versus $52.12 \pm 3.4 \mathrm{~ms} ; \mathrm{p}=0.80)$ or the change in R2* due to USPIO $\left(29.42 \pm 8.14\right.$ versus $\left.25.8 \pm 7.8 \mathrm{~s}^{-1} ; \mathrm{p}=0.43\right)$ between baseline and 3 months in patients with cardiac transplant (Figure 6.3). The myocardial biopsy 24 hours after USPIO administration revealed normal myocyte architecture with no evidence of increased numbers of inflammatory macrophages or the presence of USPIO (Figure 6.4).

\subsubsection{CARDiaC TRANSPLANT PATIENT WITH RECENTLY SUSPECTED ALLOGRAFT REJECTION}

One patient was recruited to the study 3 days following admission with non specific malaise. This was the only patient in my cohort that had a previous history of previous acute cellular rejection ( 2 years before). On this occasion, the myocardial biopsy showed grade 1a rejection (i.e. no evidence of recurrence of rejection or cellular infiltration) and the patient was reassured, but subsequently recruited to the study. This patient had the highest plasma high-sensitivity troponin I in the cohort at $71 \mathrm{ng} / \mathrm{L}$. Other blood tests were normal including white cell count $\left(4.3 \times 10^{9} / \mathrm{L}\right)$ and C-reactive protein $(<1 \mathrm{mg} / \mathrm{L})$. The $\mathrm{T} 2$ value for this subject was the highest in our cohort at $66.4 \mathrm{~ms}$ (16-segment average of $61.8 \mathrm{~ms}) . \mathrm{R} 2 *$ was $26.7 \mathrm{~s}^{-1}$ at baseline and 
$50.6 \mathrm{~s}^{-1} 24$ hours after USPIO: change in R2* was $23.8 \mathrm{~s}^{-1}$. Despite censoring this patient from analysis, patients with cardiac transplantation continued to have a higher T2 value than volunteers $(52.4 \pm 2.8$ versus $48.6 \pm 1.9 \mathrm{~ms} ; \mathrm{p}=0.0056)$. This patient did not undergo gadolinium-enhanced imaging at the request of the attending clinician due to mild renal impairment.

A

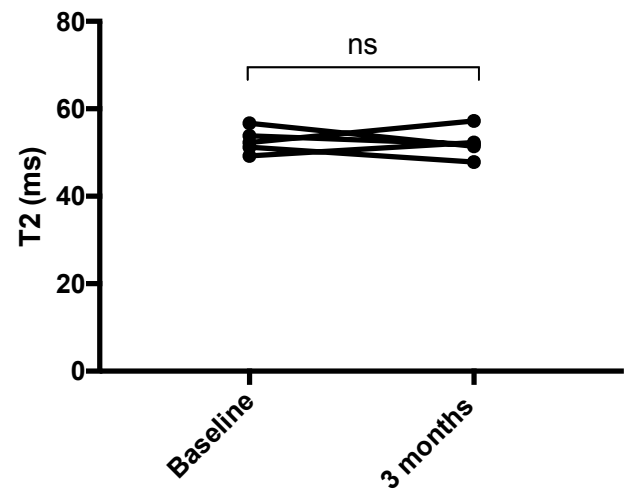

B

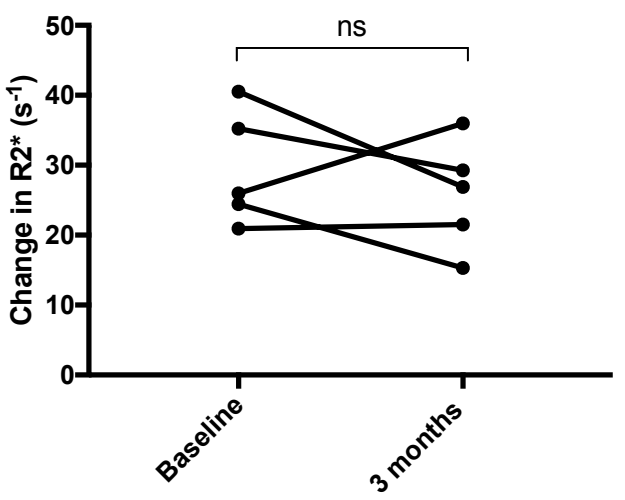

Figure 6.3. Repeated imaging in patients with cardiac transplant. There were no differences in myocardial T2 ms (A) or the change in $\mathrm{R}^{*} \mathrm{~s}^{-1}$ (B) due to USPIO between time points $(\mathrm{n}=5)$. USPIO - ultrasmall superparamagnetic particles of iron oxide.

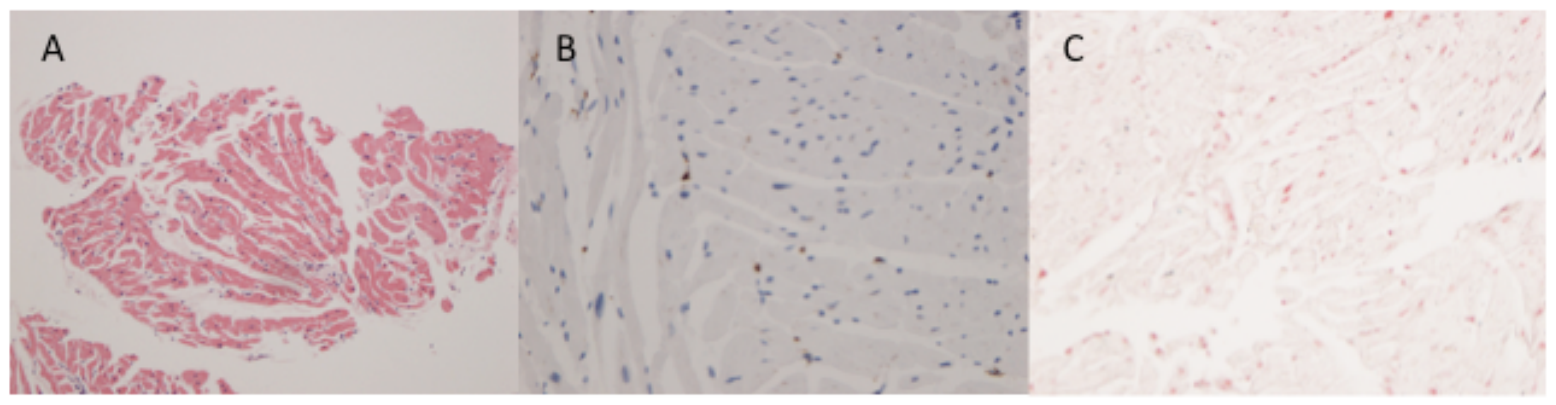

Figure 6.4. Biopsy from patient with cardiac transplant. A) Haematoxylin and eosin (x100) staining showing normal myocyte architecture with no evidence of significant inflammatory cell infiltration. B) CD68 staining (x200) showing only very few scattered macrophages (brown). C) Prussian Blue staining showing no evidence of iron. CD - cluster of differentiation. 


\subsection{DISCUSSION}

For the first time, I report the combined assessment of T2 mapping and USPIOenhanced T2* MR imaging in patients with prior cardiac transplantation and healthy volunteers. This technique is feasible, safe and well tolerated in this group of patients. I demonstrate that whilst myocardial $\mathrm{T} 2$ is increased, there is no evidence of ongoing or chronic cellular inflammatory uptake as detected by USPIO enhancement. Further assessments of these measures are now needed in patients with acute cardiac transplant rejection.

USPIO-enhanced T2* MRI has previously been used in man to assess cardiovascular inflammation in a range of different conditions [Tang et al 2009; Richards et al 2011; Alam et al 2012; Yilmaz et al 2013; Stirrat et al 2017], and preclinical models suggest this technique may be useful in assessing human patients with transplant rejection [Kanno et al 2001; Johansson et al 2002; Penno et al 2005; Wu et al 2009; $\mathrm{Wu}$ et al 2013; Alam et al 2015]. In my cohort of stable patients with cardiac transplantation, I did not detect greater USPIO enhancement within the myocardium, but this contrasted with the increased measures of myocardial T2 found in transplant patients compared to volunteers.

Why did I detect higher T2 values in transplant patients compared to controls in the absence of USPIO accumulation? There are several plausible explanations. First, chronic residual low grade inflammation or oedema without tissue-resident macrophage infiltration is possible, perhaps from lymphocytes that do not take up 
USPIO. In the absence of histological data, I cannot confirm the presence of lymphocytes, but in a stable group of patients who are clinically well this explanation appears unlikely. Second, higher T2 values in the myocardium of cardiac allografts may simply be due to increased water content, possibly related to expansion of the myocardial vasculature and blood content. The finding of greater measures of R2* (both at baseline and after USPIO), which measures iron within the blood pool, would support this mechanism. This explanation may suggest intriguing differences in the behaviour of resting myocardial vascular integrity in patients with stable cardiac allografts. Third, the relatively low field strength of the MRI scanner (1.5 T) may be contributory since it has poorer sensitivity in detecting sparse myocardial USPIO compared to higher field strength $3 \mathrm{~T}$ scanners. However, in my single myocardial biopsy, substantial numbers of macrophages were not evident. Finally, the most plausible explanation for greater measures of $\mathrm{T} 2$ in cardiac allografts may in fact reflect a change in myocyte architecture caused by myocardial fibrosis, or scarring. High T2 value is usually solely attributed to myocardial oedema, but has previously been found to inversely correlate with LV function in patients with dilated cardiomyopathy that did not have any evidence of inflammation or other reasons for myocardial oedema [Nishii et al 2014]. This may suggest higher T2 values reflect myocardial fibrosis, similar to results using T1 mapping approaches. Furthermore, higher $\mathrm{T} 2$ has also been found in patients with hepatic fibrosis [Guimaraes et al 2016]. I therefore contend that elevated $\mathrm{T} 2$ in the myocardium of cardiac allografts may reflect intrinsic changes to the cellular architecture, such as the presence of myocardial fibrosis, and not myocardial oedema in this cohort. As such, it may prove to be a further imaging biomarker of myocardial fibrosis. 
Crucially I lacked adequate access to sufficient histology or T1 mapping in order to prove whether subtle myocardial fibrosis, not detected by LGE imaging, is a causative factor in the high T2 value found. Most patients had undergone transplantation many years ago (Table 6.1) so using historical postoperative surveillance biopsies would not provide an accurate assessment of current myocyte architecture and we chose not to use these for histological correlation.

Irrespective of the aetiology, greater $\mathrm{T} 2$ in stable transplant patients is an interesting finding. Previous small studies have found that T2-weighted and T2 mapping MRI assists in diagnosis and prognosis estimation in patients with cardiac transplant rejection [Marie et al 2001; Usman et al 2012; Bonnemains et al 2014]. Following on from these studies, the DRAGET study [Bonnemains et al 2015], a large multicentre multinational study, is now prospectively recruiting patients in the first year after transplantation to assess the performance of $\mathrm{T} 2$ mapping in diagnosing transplant rejection. The findings of this study are awaited with interest, but clearly if the T2 value in myocardium of stable cardiac allografts is high, this increases the likelihood of false positive diagnoses of allograft rejection and ultimately reduces the specificity and precision of the test.

A further study by Miller et al examined 22 patients with cardiac transplantation in the early post-operative period with CMR and cardiac biopsy. Unsurprisingly, they found that myocardial $\mathrm{T} 2$ and $\mathrm{T} 1$ was higher in the patients that displayed graft rejection $(n=5)$ compared to those unaffected. In patients without biopsy evidence of graft rejection, both $\mathrm{T} 1$ and $\mathrm{T} 2$ values were significantly greater than healthy 
volunteers suggesting greater measures of myocardial oedema even in clinically well patients without biopsy evidence of inflammation. T1 and T2 measures improved over the 22 week follow-up period, but there remained significantly greater T1 values at the end of the follow up period in the non-rejecting transplant patients compared to volunteers. These findings are in keeping with my results and suggest that $\mathrm{T} 1$ and $\mathrm{T} 2$ measures may be elevated, even in 'healthy' transplant patients. It is unfortunate the Miller et al did not follow these patients for longer to see if MRI measures normalised.

One patient had very high myocardial T2 and deserves special mention. This was the only patient with a previous history of biopsy proven acute cellular rejection 2 years previously. At the time of recruitment the patient was recovering from a non specific illness and recurrent episode of rejection had been discounted on the basis of negative endomyocardial biopsies 3 days previously. Based upon the studies mentioned previously, myocardial T2 value of this magnitude suggests severe rejection. Serial imaging with T2 mapping, both prior and subsequent to this episode, would have been useful in determining the time course and fluctuation in $\mathrm{T} 2$ to assess whether it is chronically elevated, which may suggest stable myocardial fibrosis, or not. This patient in fact had T1 maps acquired as part of imaging biobank retention (the only one in my study) and septal values were elevated at $1018 \mathrm{~ms}$ (normal values 950 $21 \mathrm{~ms}$ ) [Dabir et al 2014].

Whether or not this patient had allograft rejection (with a negative biopsy) or not remains uncertain, but a clinical improvement was made with no new changes to 
medical therapy, making an episode of rejection less likely. If myocardial fibrosis and active rejection or inflammation is not the cause of elevated $\mathrm{T} 2$ then why was the T2 value so high in this patient? A high T2 value of this magnitude in the absence of rejection would cast serious doubt on the precision of T2 mapping to diagnose and exclude cardiac allograft rejection and diminish its potential utility in clinical practice. The DRAGET study will be well placed to answer this [Bonnemains et al 2015].

\subsubsection{LiMitATIONS}

There are some limitations that deserve mention. Participant numbers were small, but sufficient for a pilot study testing the feasibility of USPIO-enhanced MRI in this population. From my recent work in patients with myocardial infarction, I know that this technique works, and is capable of identifying and tracking myocardial macrophage accumulation [Stirrat et al 2016]. In this study, myocardial macrophage infiltration is either not a chronic feature of transplantation or at levels too low to be detected.

My aim was to recruit a range of patients with cardiac transplantation, from chronic stable patients, to patients with suspected allograft rejection. Unfortunately, I was only able to recruit one patient with recently suspected transplant rejection, and this was excluded clinically, with the remaining patients being stable and well. Small study numbers and absence of patients with florid transplant rejection clearly limit my findings and this technique now needs to be tested in patients with proven acute transplant rejection. 


\subsubsection{Conclusion}

I have shown that stable patients with cardiac transplantation have greater measures of myocardial T2, which may suggest myocardial oedema or fibrosis, compared with control volunteers. Ferumoxytol-enhanced MR imaging does not add clinically relevant information in this group of stable patients but may provide additional information in the setting of macrophage-driven acute cellular transplant rejection. 


\section{CHAPTER 7}

\section{CONCLUSION AND FUTURE DIRECTIONS}




\subsection{SUMMARY}

Novel molecular and cellular imaging techniques capable of non-invasively examining metabolic and biological processes would be valuable in a range of cardiovascular diseases to aid understanding of disease pathogenesis, provide diagnostic information, predict disease progression, and guide therapeutic intervention. It would therefore be a major clinical advance if a reliable non-invasive imaging technique capable of detecting tissue inflammation could be developed.

Magnetic resonance imaging has a number of advantages including excellent discrimination of soft tissue components, good spatial resolution and in particular, absence of ionising radiation, an important consideration if serial imaging is envisaged. T2* imaging for assessing iron accumulation has successfully been used for over a decade in diagnosing and grading the severity of iron accumulation in patients with transfusion-dependent thalassaemia major, helping provide serial disease monitoring and guide therapy that improves prognosis [Anderson et al 2001; Anderson et al 2004]. In this disease, T2* MRI has improved sensitivity in detecting early myocardial iron accumulation compared to serum iron sampling [Anderson et al 2001]. T2* MRI in the assessment of iron accumulation is easily quantifiable, well validated, highly reproducible, clinically robust, and is achievable in a single breathhold [Westwood et al 2003a; Westwood et al 2003b; Tanner et al 2006; Carpenter et al 2009; Kirk et al 2010; Carpenter et al 2011]. 
Iron oxide nanoparticles initially generated interest as an MRI blood pool contrast agent, but recent studies have revealed them to be an effective tracer, highlighting inflammatory tissue macrophages, and clinical applications have started to emerge [Trivedi 2004; Tang et al 2006; Trivedi 2006; Tang et al 2009; Richards et al 2011; Alam et al 2012; Yilmaz et al 2013; McBride et al 2015]. Following administration, USPIO are small enough to migrate passively across the vessel wall [Wang et al 2001] especially in regions of inflamed vasculature where there is loss of endothelial integrity. Cells of the reticuloendothelial system, particularly tissue macrophages, identify, internalise and concentrate iron oxide nanoparticles generating markedly reduced T2 and T2* MRI relaxation times. Thus USPIO-enhanced MRI can identify tissue-resident macrophage activity and identify cellular inflammation within tissues.

In the studies presented in my thesis, I first determined a range of normal T2* values at 1.5 Tesla and 3 Tesla in healthy volunteers before and after USPIO, detailing methodology used and describing commonly encountered imaging problems (Chapter 3). I went on to assess whether USPIO-enhanced T2* MRI can detect and serially monitor myocardial inflammation after myocardial infarction using single and repeated USPIO administration (Chapter 4). Thereafter I compared USPIO enhancement in the myocardium of volunteers with patients affected with acute myocarditis (Chapter 5) and prior cardiac transplantation (Chapter 6). 


\subsection{SUMMARY OF THESIS FINDINGS}

\subsubsection{A RANGE OF NORMAL TISSUE VALUES FOR USPIO-ENHANCED T2* MRI WAS}

DETERMINED AT 1.5 TESLA AND 3 TESLA

Following USPIO administration in healthy volunteers, USPIO was detected by changes in R2* from baseline at 24 hours in myocardium, skeletal muscle, kidney, liver, spleen and blood at $1.5 \mathrm{~T}$, and myocardium, kidney, liver, spleen, blood and bone at $3 \mathrm{~T}$. Myocardial changes in R2* due to USPIO were $26.5 \pm 7.3 \mathrm{~s}^{-1}$ at $1.5 \mathrm{~T}$, and $37.2 \pm 9.6 \mathrm{~s}^{-1}$ at $3 \mathrm{~T}$. Tissues showing greatest ferumoxytol enhancement were the reticuloendothelial system: the liver, spleen and bone marrow at both 1.5 and $3 \mathrm{~T}$.

Tissues with small increases in R2* after USPIO (i.e. less than that of the blood pool) can be explained by detection of USPIO within the intravascular space and include skeletal muscle (at $1.5 \mathrm{~T}$ only), myocardium and kidney. In contrast, R2* changes due to USPIO that are greater than the blood pool must be due to accumulation of USPIO, either through iron storage, uptake by macrophages or other phagocytes, or sequestered within tissue interstitium. As the most pronounced R2* changes were seen in the spleen, liver and bone marrow: organs of the reticuloendothelial system, it would appear likely that USPIO is incorporated quickly into tissue-resident phagocytes and macrophages.

\subsubsection{SOlutions TO COMMONLY ENCOUNTERED PROBLEMS WITH USPIO-} ENHANCED T2* MR IMAGING WERE DETAILED

Artefacts are commonly encountered with $\mathrm{T} 2 *$ imaging and made data analysis 
challenging. Post USPIO contrast artefacts at the blood pool to myocardial interface were commonly seen and needed careful exclusion when selecting myocardial ROI. This limited the assessment of USPIO accumulation at the endocardium. Similarly, blooming artefacts from nearby organs with high iron or blood pool USPIO content, such as lung and liver, commonly created signal deficits within the myocardium. In this situation, examination of $\mathrm{T} 2 *$ decay curves and excluding echo times influenced by artefact aided $\mathrm{T} 2 *$ decay curve fitting.

The advantage of MRI mapping techniques is that visual assessment and objective quantification can be made using the same image, and these are now entering clinical practice. However based on my experiences, I would recommend caution in interpreting maps alone. Signal attenuation seen on the T2* map may be interpreted as tissue USPIO accumulation, but may be due to blooming artefact from nearby susceptibility effects, and close examination of the $\mathrm{T} 2 *$ decay curve, and individual echoes is suggested in order to distinguish accurately between tissue USPIO accumulation and artefact. Setting a decay curve fitting acceptability threshold $\left(\mathrm{r}^{2}\right)$, as I did, helps exclude areas grossly affected by artefact. In practice however, regions with a seemingly acceptable $\mathrm{r}^{2}$ may still be heavily influenced by artefact and there is no acceptable substitute for visualising decay curves and individual echo images.

Finally, applying tissue specific echo times tailored to the expected T2* value should be used in order to achieve the most accurate decay curves possible. 


\subsubsection{ULTRASMALL SUPERPARAMAGNETIC PARTICLES OF IRON OXIDE-ENHANCED}

T2* MR IMAGING CAN DETECT MYOCARDIAL MACROPHAGES AFTER MYOCARDIAL INFARCTION

Following a single USPIO infusion in the first week after MI, USPIO enhancement is seen in all regions of the myocardium, but greatest within the infarct zone, after 24 hours. USPIO enhancement is also observed until days 4-9 in bone marrow, and until days 13-21 in the liver and spleen. Therefore the duration of USPIO enhancement is longest in the mononuclear phagocyte rich reticuloendothelial system. It is likely that resident phagocytes within these organs ingest USPIO from circulating blood. Efflux of myocardial macrophages containing USPIO may also contribute to the reticuloendothelial system T2* signal seen after MI.

\subsubsection{USPIO-ENHANCED T2* MR IMAGING CAN SERIALLY MONITOR MYOCARDIAL}

\section{MACROPHAGES AFTER MI}

Following repeated doses, USPIO uptake in the infarct zone peaked at days 2-3, and greater USPIO uptake was detected in the infarct zone compared to remote myocardium until days 10-16. In contrast, T2-defined myocardial oedema peaked at days 3-9 and remained increased in the infarct zone throughout the 3-month follow up period.

I have demonstrated a discontinuity between the prolonged (up to 3 months) T2defined time course of tissue oedema, and the brief (first 2 weeks) T2*-defined time limited cellular inflammation. This observed USPIO uptake in the infarct zone for up to 2 weeks following MI is consistent with data from previous studies demonstrating 
that tissue-resident macrophages are active and predominate within the infarcted myocardium in the first 2 weeks after MI [Tsujioka et al 2009; Nahrendorf et al 2010]. Furthermore, the finding of myocardial oedema within the infarct zone at 3 months after MI may at first appear surprising, but long lasting myocardial oedema, often up to 6 months after MI, has previously been described [Nilsson et al 2001; Dall'Armellina et al 2011].

\subsubsection{USPIO ENHANCEMENT IN THE MYOCARDIUM OF PATIENTS WITH ACUTE} MYOCARDITIS

There was no demonstrable difference in USPIO enhancement between patients and volunteers even within regions displaying late gadolinium enhancement. However, there was greater T2 value in regions of LGE in patients with myocarditis when compared to healthy volunteer myocardium suggestive of myocardial oedema. This suggests that tissue-resident macrophages do not provide a substantial contribution to the myocardial inflammation in this condition, and that USPIO-enhanced MRI does not provide additional clinically relevant information to LGE and T2 mapping MRI in this condition.

Imaging after 3 months in myocarditis patients was repeated and imaging evidence of recovery was found: a reduction in volume of LGE, a reduction in T2 value in regions displaying LGE, and improvement in ejection fraction.

7.2.6 USPIO ENHANCEMENT IN THE MYOCARDIUM OF PATIENTS WITH PRIOR CARDIAC TRANSPLANTATION 
I determined that there were no differences in USPIO uptake in the myocardium of patients with transplantation compared to healthy volunteers. Therefore USPIOenhanced MRI is normal and stable over time suggesting the absence of chronic macrophage-driven cellular inflammation. However, in contrast, myocardial T2 values were higher in patients with cardiac transplant versus healthy volunteers. This finding is intriguing and there are several explanations. Chronic residual low grade inflammation or oedema without tissue-resident macrophage infiltration (or inadequate MRI magnetic strength to detect sparse USPIO-laden macrophages) is possible, but the raised T2 values found may simply relate to expansion of the myocardial vasculature after cardiac transplantation. Finally, the most plausible explanation for greater measures of $\mathrm{T} 2$ in cardiac allografts may in fact reflect a change in myocyte architecture caused by myocardial fibrosis, or scarring. Crucially I lacked adequate access to sufficient histology or T1 mapping in order to prove whether subtle myocardial fibrosis, not detected by LGE imaging, is a causative factor in the high $\mathrm{T} 2$ value found.

\subsection{FUTURE DIRECTIONS}

The conclusions of this thesis raises several questions regarding whether USPIOenhanced cardiac magnetic resonance will enter clinical practice to aid diagnosis, improve risk stratification and serial monitoring in inflammatory conditions driven by macrophages. I will discuss my ideas for future research. 


\subsubsection{IRON NANOPARTICLES TO AID DETECTION OF STEM CELLS}

Current research endeavours are increasingly focusing on stem cell therapy that attempts to restore function to organs with little or no regenerative capacity. This follows the discovery that pluripotent progenitor cells bear the capacity to differentiate into mature cardiac cells. There is therefore a need to non-invasively track the distribution and viability of administered stem cells. Cell labelling with iron nanoparticles has been proposed as it is relatively simple, fast, inexpensive and is well described, although there are few published studies using this technique. Tracking of stem cells labelled with iron nanoparticles using MR imaging is still at the preclinical stage but offers high spatial resolution, excellent soft tissue detail and avoids exposure to ionising radiation.

Küsterman et al [2005] labelled cardiac progenitor cells expressing enhanced green fluorescent protein (EGFP) with ferumoxtran-10 (USPIO) and implanted the cells into murine myocardium injured through either cryocoagulation or coronary artery ligation. Colocalisation of USPIO and stem cells was confirmed by comparing the high-resolution MRI images with Prussian Blue staining (for iron) on histology, and immunohistochemistry for EGFP.

Chapon et al [2009] combined MR imaging and positron emission tomography (PET), to track transplanted stem cells after myocardial infarction in rats providing anatomical, physiological and metabolic tissue information. They conjugated rat bone marrow-derived stem cells to CLIO. Stem cells were successfully detected on 
MRI and histology for the 6-week duration of the study, although increased myocardial metabolic activity detected by PET (18F-fluorodeoxyglucose uptake) was only seen in the first week post stem cell implantation. This may represent a short-lived improvement in tissue viability after stem cell transplantation, but more likely may be due to early, localised inflammation from intramyocardial stem cell injection settling over time. A trend to an improvement in cardiac function was found after stem cell implantation, although this did not reach statistical significance.

Imaging of stem cells using larger iron particles has also been conducted with success [Kraitchman 2003; Chung et al 2011; Lu et al 2012]. Of particular interest, Lu et al [2012] recently investigated the effects of culprit vessel intracoronary infusion of SPIO-labelled (ferucarbotran) autologous bone marrow-derived mesenchymal stem cells (MSCs) after acute myocardial infarction in pigs. One week after MI, SPIO-labelled MSCs were infused via the culprit coronary vessel and compared to a control infusion of phosphate buffer saline. The pigs were imaged immediately before and 3 days after cell transplantation. Detection of SPIO-labelled MSCs was observed on T2* MRI after cell transplantation. However, pronounced reduction in $\mathrm{T}^{*}$ values was found in the spleen, liver and kidney with immunohistochemistry confirming this to be the SPIO-MSC conjugate, suggesting the majority of the infused cells escaped into the systemic circulation, and were subsequently sequestered in the organs of the reticuloendothelial system. The major disadvantage of labelling MSCs in vitro prior to injection (as in this study), is that subsequent MRI would not detect any 'offspring' MSCs (having no SPIO attached) should the stem cell successfully proliferate. 
As a solution to this problem of in vitro SPIO-labelled MSCs being unable to detect newly created stem cells, Chung et al [2011] made use of a clever reporter geneexpressing antigen on the surface of the embryonic stem cells (ESCs). They separately injected SPIO-conjugated to a monoclonal antibody (SPIO-MAb) that targeted the antigen expressed by the reporter gene, allowing in vivo MRI quantification of viability, proliferation and teratoma formation of transplanted ESCs and their offspring. The advantage of this technique over direct stem cell labelling is that proliferated stem cells can now be tracked. Signal is enhanced when the stem cell successfully divides thus allowing successful detection of newly proliferated stem cells.

These studies above suggest that iron nanoparticle enhanced MRI may be well placed to assess the viability and proliferation of implanted stem cells in future research. Clinical trials using this technique have yet to emerge but this may happen in the near future. Before this can take place, MRI detection limits for specific stem cell, iron nanoparticle, MRI scanner combinations are likely to be different and would have to be predetermined, although work has already begun on this [Mathiasen et al 2013].

\subsubsection{IRON NANOPARTICLES TO AID DETECTION OF APOPTOSIS}

Targeting of various pathophysiological processes of interest becomes possible when contrast agents, including iron oxide particles, are conjugated to a specific antibody or peptide [McAteer et al 2010]. Like stem cells described above, iron nanoparticles can facilitate imaging of apoptosis, or cell death. Non-invasive detection of early, 
reversible apoptosis might be useful clinically. During apoptosis, the phospholipid phosphatidylserine is externalised on the cell surface. Annexin V is an endogenous protein that binds with high affinity to phosphatidylserine, and when conjugated to CLIO, has successfully been able to identify apoptotic cells in vitro using MR imaging [Schellenbeerger et al 2002]. Sosnovik et al [2005] used this particle as a foundation to create a multimodality imaging nanoparticle when conjugated to cyanine 5.5 dye (AnxCLIO-Cy5.5) allowing co-identification of apoptosis with fluorescence microscopy and T2* MRI. In a murine model of ischaemia-reperfusion injury, a reduction in signal intensity on $\mathrm{T} 2 *$ MRI was observed in reperfused regions with AnxCLIO-Cy5.5 but not with unlabelled CLIO-Cy5.5. The area of signal loss correlated with the region of contractile dysfunction seen on MRI, and with areas of increased fluorescence intensity on near infrared fluorescence confirming that this was correctly identifying ischaemic myocardium.

The same group then developed a novel dual contrast molecular MRI probe to help identify ischaemic 'at-risk', but viable, myocardium after ischaemia-reperfusion injury [Sosnovik et al 2009]. Using the same AnxCLIO-Cy5.5 particle described above, they identified cellular apoptosis in mice on MRI 4-6 hours after ischaemiareperfusion injury. However, to illustrate necrosis, gadolinium was also administered with late enhancement MRI. Gadolinium accumulates in regions of cell necrosis, with cell wall rupture and expansion of the extracellular volume. In apoptosis alone, the cell wall remains intact, and gadolinium does not accumulate. Interestingly, they found only $21 \%$ of regions displaying apoptotic myocardium (accumulation of AnxCLIO5.5) had evidence of necrosis (defined by late gadolinium enhancement). 
This suggests that the majority of apoptotic cardiomyocytes remain viable 4-6 hours after ischaemia-reperfusion injury, and this was confirmed on fluorescence microscopy and immunohistochemistry revealing large numbers of apoptotic, but potentially viable, cardiomyocytes in the at-risk area (AnxCLIO-Cy5.5 positive, gadolinium negative areas). This imaging technique may provide an ideal imaging platform to trial strategies to salvage apoptotic, but not yet necrotic, myocardium after an ischaemic insult.

Perhaps the most interesting role for iron nanoparticles in assessing apoptosis was illustrated by Dash et al [2011] in the setting of chemotherapy induced cardiotoxicity. They conjugated SPIO to recombinant human Annexin V (ANX-SPIO), and examined doxorubicin-induced cardiotoxicity in rat myocytes, cardiac fibroblasts, mesenchymal stem cells and also in mice in vivo. Areas of signal attenuation on T2* MRI correlated with iron accumulation and regions of apoptosis on histology, although myocardial structure appeared normal suggesting the detection of early apoptosis only. Perhaps more importantly, they demonstrated that treatment with A61603 (an alpha-1 adrenoreceptor agonist that prevents doxorubicin-induced cardiomyopathy), reversed apoptosis on histology and eliminated the ANX-SPIO signal on MRI. These findings suggest that this technique may have the ability to detect early myocyte injury, such as in patients with early chemotherapy-induced cardiotoxicity, at a stage where effective treatment can be delivered. 


\subsubsection{ASSESSMENT OF USPIO ENHANCEMENT WITH T2 MAPPING}

Imaging assessment of USPIO has historically been achieved with T2* MRI. T2* MRI measures the 'true' $\mathrm{T} 2$ of tissue in addition to magnetic field inhomogeneities due to imperfections of the scanner magnet itself or magnetic susceptibility effects within the patient or placed within the magnetic field (such as USPIO). $\mathrm{T} 2 *$-weighted imaging therefore requires high uniformity of the magnetic field. In spin echo T2 imaging, a refocusing pulse removes dephasing due to magnetic field inhomogeneities and susceptibility effects. In gradient echo T2* MRI there is no such refocusing pulse and the susceptibility effects of USPIO can be measured by reduction in $\mathrm{T} 2 *$. Gradient echo has the advantages of shorter acquisition times but suffers from relatively reduced signal, artefacts in regions of tissue interfaces, and generally poorer image quality compared to T2 spin echo sequences.

Accumulation of USPIO particles has two major effects: a predominant susceptibility effect mentioned above, but also direct $\mathrm{T} 2$ shortening caused by local field inhomogeneities that promotes transverse relaxation [Sanjay et al 2004]. As such, UPSIO can be detected on both $\mathrm{T} 2$ and $\mathrm{T} 2 *$ imaging, but $\mathrm{T} 2 *$ imaging is more sensitive in detecting USPIO than T2 imaging [Kaim et al 2002; Simon et al 2006]. The reduced sensitivity of $\mathrm{T} 2$ imaging in detecting USPIO may be in part due to prolonged T2 values of oedematous tissue opposing the USPIO-lowering T2 effects, especially when assessing areas of tissue inflammation. Observations of our data throughout this thesis show that the spread of $\mathrm{T} 2 *$ values, even in healthy tissue, is greater than that seen in T2 imaging. This is probably explained by artefact and poorer image quality of gradient echo $\mathrm{T} 2 *$ sequences compared to $\mathrm{T} 2$ imaging 
described above. Larger spread of data within normal healthy tissue makes it more difficult to detect threshold levels of USPIO accumulation within inflamed tissue. This raises the question of whether $\mathrm{T} 2$ imaging could be used to assess USPIO accumulation. The answer lies with whether the sensitivity of T2 imaging for detecting tissue USPIO is good enough to swing the balance in favour of T2 imaging. I am unable to answer this with results in this thesis but this could be readily addressed with pre and post USPIO T2 mapping.

\subsubsection{NOVEL INFLAMMATORY IMAGING WITH COMBINED MRI/PET}

${ }^{18}$ F-Fluorodeoxyglucose positron emission tomography (FDG-PET) has been widely used to measure myocardial inflammation. As shown in this thesis, cardiac MRI with late gadolinium enhancement has become well established for detecting and differentiating myocardial injury in a range of conditions. The development of simultaneous PET/MR imaging now allows combination of these techniques alongside function assessment with advantages in terms of improved image co-registration and interpretation, and reduced radiation exposure.

Sarcoidosis is a multisystem inflammatory disease. There is cardiac involvement in up to $27 \%$ of sarcoid cases and complications include atrial and ventricular arrhythmias, congestive cardiac failure and sudden cardiac death [Roberts et al 1977; Yazaki et al 2001; Banba et al 2007; Kandolin et al 2011]. The core inflammatory cells of sarcoid granulomas include monocytes and macrophages [Mitchell et al 1977] and an imaging technique targeting these cells would be ideally placed to establish the diagnosis and to assess disease activity. 
Hybrid MRI/PET has recently been found to be useful in detecting active myocardial sarcoidosis and acute myocarditis [Abgral et al 2017; Dweck et al 2017]. Patients with inflammatory cardiac conditions are now being recruited in our centre and it will be interesting to follow these results to assess the success of this novel technique in diagnosing and monitoring inflammatory cardiac conditions.

As part of this series of research studies, we intended to recruit patients with active cardiac sarcoidosis. Despite having three recruiting centres across the UK (including the national sarcoid centre at the Royal Brompton hospital) we struggled to recruit sufficient numbers of patients. Twelve patients with systemic sarcoidosis were recruited and only 2 had evidence of active or previous myocardial involvement. This was insufficient for conclusions to be drawn and is not discussed further in this thesis.

\subsubsection{THINGS I WOULD DO DIFFERENTLY}

Completing a thesis such as this gives the opportunity for reflection. Naturally, there are things during the course of my research that, given the opportunity again, I would do slightly differently. I feel it is important to mention these at the end of a piece of work such as this.

After myocardial infarction, I was able to detect macrophages in the infarct region during the expected time period of two weeks after myocardial infarction. However, as discussed in Chapter 4, two specific subsets of macrophages that have divergent functions operate during this period. To help guide future research studies, it would 
have been helpful to determine whether or not both subsets of macrophages ingest USPIO. The time course of USPIO enhancement seen suggests both subgroups ingest USPIO, but the collection of peripheral blood for flow cytometry at the time of MRI would provide further information on circulating macrophages at the time of MRI.

As part of a pilot study for an undergraduate medical student study, we collected blood for flow cytometry from a small number of patients $(n=9)$ at some, but not all, of the MRI time points, Without access to original data, I was unable to include this into the results of this thesis, however we saw the expected early peak in classical monocytes with a later peak in non-classical monocytes. Collecting and measuring circulating monocytes in all patients at all MRI time points may help determine whether both subgroups ingest USPIO, and whether a correlation exists been particular monocyte subgroups and USPIO accumulation measured on MRI.

In this chapter I have already mentioned the potential for USPIO measurement by T2 mapping. However T1 mapping is entering clinical practice as an MRI marker for myocardial fibrosis and has also recently been proposed as a technique to assess intra cardiac iron deposition with good correlation with myocardial T2*, and may even have enhanced sensitivity in detecting myocardial iron compared to $\mathrm{T} 2 *$ [AbdelGadir et al 2016]. However due to an already lengthy MRI scanning protocol, I was not able to add pre and post USPIO T1 mapping sequences to the protocol. This would be a useful addition to future research studies using USPIO. 
Running a multicenter imaging study in 3 sites in the UK, with the majority of participants recruited in the final year, meant that some areas I had considered for research were never reached. Further analysis could be carried out on stored blood samples, particularly for serial measurements of inflammatory markers and mediators, as well as biomarkers such as BNP. This could be considered at a later date.

Finally, it is regrettable that I was not able to recruit sufficient numbers of patients with confirmed active cardiac transplant rejection and active cardiac sarcoidosis. The utility of USPIO-enhanced MRI in these groups of patients is unknown.

\subsection{CLINICAL PERSPECTIVE}

There is an unmet clinical need for reliable, non-invasive imaging techniques capable of detecting disease processes at a molecular and cellular level. Successful development of such techniques would improve diagnosis by detecting early disease states, help understand disease pathophysiology, provide serial monitoring and risk stratification, and help guide therapeutic intervention. For these reasons, there is currently considerable research interest into the development of novel molecular imaging techniques.

Magnetic resonance imaging has the particular advantage of having excellent spatial resolution and being free from ionising radiation. Following administration, USPIO is taken up by macrophages and can be detected on T2* MRI. Therefore USPIOenhanced MRI is able to detect tissue-resident macrophages and provide information 
on cellular and tissue inflammation in inflammatory myocardial diseases mediated by macrophage infiltration.

Following myocardial infarction, maladaptive macrophage accumulation can lead to adverse functional recovery. USPIO-enhanced MRI may be of value in diagnosing active myocardial inflammation and quantifying macrophage accumulation. Ultimately, this technique may aid risk stratification after MI, provide serial assessment, and act as a platform on which to assess existing and novel therapeutic anti-inflammatory interventions that might modify the inflammatory process.

Acute myocarditis and cardiac transplant rejection, other conditions where inflammation affects the myocardium, cause considerable clinical concern and rapid assessment is vital. These conditions remain a diagnostic challenge and suffer from a lack of simple definitive diagnostic tests, where gold standard cardiac biopsies are unreliable, associated with risk and are rarely carried out. Following my experiments, USPIO-enhanced MRI provided no additional diagnostic value in acute myocarditis or in assessing stable patients with cardiac transplantation, and that tissue-resident macrophages do not appear to be a major contributor to the cellular inflammation in these conditions. Of considerable interest, stable patients with cardiac transplantation have increased resting myocardial T2 values, consistent with myocardial oedema or fibrosis. This suggests that T2 assessment of myocardial oedema alone in this condition may lead to false positive diagnoses of active tissue inflammation and cause unnecessary treatment interventions. Results from my experiments could not determine whether USPIO-enhanced MRI is able to diagnose and monitor acute 
cardiac allograft rejection, findings suggested in preclinical models, and this patient group requires further examination [Kanno et al 2001; Johansson et al 2002; Penno et al 2005; Wu et al 2009; Wu et al 2013].

Although USPIO-enhanced MRI failed to detect tissue macrophages in clear cases of acute myocarditis, this does not mean it provides no value. We know this technique can detect macrophages after MI. It may be simply that there were no macrophages to detect in acute myocarditis. Further assessments in patients with macrophage infiltration of the myocardium should now take place; such as acute cardiac transplant rejection. Furthermore, USPIO-enhanced MRI appears well placed to be involved in regenerative medicine in assessing the viability, distribution and proliferation after stem cell therapy, and also in detecting preclinical stages of chemotherapy-induced cardiotoxicity, where rapid detection and early intervention may improve clinical outcomes for cancer patients.

Continued refinements to image acquisition and analysis processes, with particular emphasis on reducing $\mathrm{T} 2 *$ imaging artefacts will ensure reliable and robust quantification of tissue enhancement. T2 mapping in the assessment of USPIO should also be considered.

In summary, I have outlined and developed robust methodologies, and demonstrated current and future clinical applications for USPIO-enhanced MRI to assess myocardial macrophage-driven myocardial inflammation. This technique has the 
potential as an invaluable clinical tool, and results from future studies will determine whether it will be incorporated into routine clinical practice. 


\section{REFERENCES}




\section{REFERENCES}

Abdel-Aty H, Zagrosek A, Schulz-Menger J et al. Delayed enhancement and T2weighted cardiovascular magnetic resonance imaging differentiate acute from chronic myocardial infarction. Circulation 2004;109:2411-2416.

Abdel-Gadir A, Vorasettakarnkij Y, Ngamkasem H et al. Ultrafast Magnetic Resonance Imaging for Iron Quantification in Thalassemia Participants in the Developing World. The TIC-TOC study. Circulation 2016; 134:432-434

Abgral R, Dweck MR, Trivieri MG et al. Clinical utility of combined FDG-PET/MR to assess myocardial disease. JCMG 2017;10:594-597.

Alam SR, Lewis SC, Zamvar V et al. Perioperative elafin for ischaemia-reperfusion injury during coronary artery bypass graft surgery: a randomised-controlled trial. Heart 2015;101:1639-1645.

Alam SR, Shah ASV, Richards J et al. Ultrasmall superparamagnetic particles of iron oxide in patients with acute myocardial infarction: Early clinical experience. Circ Cardiovasc Imaging 2012;5:559-565.

Alam SR, Tse GH, Stirrat C et al. Nanoparticle enhanced MRI scanning to detect cellular inflammation in experimental chronic renal allograft rejection. Int $J \mathrm{Mol}$ Imaging 2015;2015(5):507909-8.

Allkemper T, Bremer C, Matuszewski L, Ebert W, Reimer P. Contrast-enhanced blood-pool MR angiography with optimized iron oxides: effect of size and dose on vascular contrast enhancement in rabbits. Radiology 2002;223(2):432-438.

Amano Y, Herfkens RJ, Shifrin RY, Alley MT, Pelc NJ. Three-dimensional cardiac cine magnetic resonance imaging with an ultrasmall superparamagnetic iron oxide blood pool agent (NC100150). J Magn Reson Imaging 2000;11(2):81-86.

Anderson LJ, Holden S, Davis B et al. Cardiovascular T2-star (T2*) magnetic resonance for the early diagnosis of myocardial iron overload. Eur Heart J 2001; 22(23):2171-2179.

Anderson LJ, Westwood MA, Holden S et al. Myocardial iron clearance during reversal of siderotic cardiomyopathy with intravenous desferrioxamine: a prospective study using T2* cardiovascular magnetic resonance. Br J Haematol 2004;127:348355 .

Aretz HT, Billingham ME, Edwards WD et al. Myocarditis. A histopathologic definition and classification. Am J Cardiovasc Pathol 1987;1:3-14.

Arslan F, de Kleijn DP, Pasterkamp G. Innate immune signalling in cardiac ischaemia. Nat Rev Cardiol. 2011;8:292-300. 
Baba $\mathrm{T}$, Ishizu A, Iwasaki $\mathrm{S}$ et al. CD4+/CD8+ macrophages infiltrating at inflammatory sites: a population of monocytes/macrophages with a cytotoxic phenotype. Blood 2006;107(5):2004-2012.

Banba K, Kusano KF, Nakamura K et al. Relationship between arrhythmogenesis and disease activity in cardiac sarcoidosis. Heart Rhythm 2007;4:1292-1299.

Barin JG, Rose NR, Ciháková D. Macrophage diversity in cardiac inflammation: a review. Immunobiology 2012;217(5):468-475.

Bernd H, De Kerviler E, Gaillard S, Bonnemain B. Safety and tolerability of ultrasmall superparamagnetic iron oxide contrast agent: comprehensive analysis of a clinical development program. Invest Radiol 2009;44(6):336-342.

Berry CC, Wells S, Charles S, Curtis ASG. Dextran and albumin derivatised iron oxide nanoparticles: influence on fibroblasts in vitro. Biomaterials 2003;24(25):4551-4557.

Berry CC, Wells S, Charles S, Aitchison G, Curtis A. Cell response to dextranderivatised iron oxide nanoparticles post internalisation. Biomaterials 2004 25(23):5405-5413.

Bjerner T, Ericsson A, Wikström $\mathrm{G}$ et al. Evaluation of nonperfused myocardial ischemia with MRI and an intravascular USPIO contrast agent in an ex vivo pig model. J Magn Reson Imaging 2000;12(6):866-872.

Bonnemains L, Villemin T, Escanye J-M et al. Diagnostic and prognostic value of MRI T2 quantification in heart transplant patients. Transpl Int 2014;27:69-76.

Bonnemains L, Cherifi A, Girerd N, Odille F, Felblinger J. Design of the DRAGET Study: a multicentre controlled diagnostic study to assess the detection of acute rejection in patients with heart transplant by means of T2 quantification with MRI in comparison to myocardial biopsies. BMJ Open 2015;5:e008963.

Bourrinet $\mathrm{P}$, Bengele $\mathrm{HH}$, Bonnemain $\mathrm{B}$ et al. Preclinical safety and pharmacokinetic profile of ferumoxtran-10, an ultrasmall superparamagnetic iron oxide magnetic resonance contrast agent. Invest Radiol 2006;41(3):313-324.

Brisset J-C, Desestret V, Marcellino S et al. Quantitative effects of cell internalization of two types of ultrasmall superparamagnetic iron oxide nanoparticles at $4.7 \mathrm{~T}$ and $7 \mathrm{~T}$. Eur Radiol 2010;20(2):275-285.

Bunce NH, Moon JC, Bellenger NG et al. Improved cine cardiovascular magnetic resonance using Clariscan (NC100150 injection). J Cardiovasc Magn Reson 2001;3(4):303-310.

Caforio AL, Goldman JH, Haven AJ et al. Circulating cardiac-specific autoantibodies as markers of autoimmunity in clinical and biopsy-proven myocarditis. The Myocarditis Treatment Trial Investigators. Eur Heart J. 1997;18(2):270. 
Caforio ALP, Pankuweit S, Arbustini E et al. Current state of knowledge on aetiology, diagnosis, management, and therapy of myocarditis: a position statement of the European Society of Cardiology Working Group on Myocardial and Pericardial Diseases. Eur Heart J 2013;34:2636-2648.

Canet E, Revel D, Forrat R et al. Superparamagnetic iron oxide particles and positive enhancement for myocardial perfusion studies assessed by subsecond T1-weighted MRI. Magn Reson Imaging 1993;11:1139-1145.

Carpenter J-P, He T, Kirk $\mathrm{P}$ et al. Calibration of myocardial iron concentration against T2-star cardiovascular magnetic resonance. J Cardiovasc Magn Reson 2009;11(Suppl 1):1-2.

Carpenter J-P, He T, Kirk $\mathrm{P}$ et al. On $\mathrm{T} 2 *$ magnetic resonance and cardiac iron. Circulation 2011;123:1519-1528.

Cerqueira MD, Weissman NJ, Dilsizian V et al. Standardized myocardial segmentation and nomenclature for tomographic imaging of the heart: A statement for healthcare professionals from the Cardiac Imaging Committee of the Council on Clinical Cardiology of the American Heart Association. J Am Soc Echocardiography 2002;15:463-467.

Chan JK, Roth J, Oppenheim et al. Alarmins: awaiting a clinical response. $J$ Clin Invest. 2012;122:2711-2719.

Chapon C, Jackson JS, Aboagye EO, Herlihy AH, Jones WA, Bhakoo KK. An in vivo multimodal imaging study using MRI and PET of stem cell transplantation after myocardial infarction in rats. Mol Imaging Biol 2009;11(1):31-38.

Chung J, Kee K, Barral JK et al. In vivo molecular MRI of cell survival and teratoma formation following embryonic stem cell transplantation into the injured murine myocardium. Magn Reson Med 2011;66:1374-1381.

Clément O, Siauve N, Cuénod CA, Frija G. Liver imaging with ferumoxides (Feridex): fundamentals, controversies, and practical aspects. Top Magn Reson Imaging 1998;9(3):167-182.

Cooper LT, Baughman KL, Feldman AM et al. The Role of endomyocardial biopsy in the management of cardiovascular disease: a scientific statement from the American Heart Association, the American College of Cardiology, and the European Society of Cardiology endorsed by the Heart Failure Society of America and the Heart Failure Association of the European Society of Cardiology. J Am Coll Cardiol 2007;50(19):1914-1931.

Dabir D, Child N, Kalra A et al. Reference values for healthy human myocardium using a T1 mapping methodology: results from the International T1 Multicenter cardiovascular magnetic resonance study. J Cardiovasc Magn Reson 2014;16:69.

Dall'Armellina E, Karia N, Lindsay AC et al. Dynamic changes of edema and late gadolinium enhancement after acute myocardial infarction and their relationship to 
functional recovery and salvage index. Circ Cardiovasc Imaging 2011;4:228-236.

Dash R, Chung J, Chan T et al. A molecular MRI probe to detect treatment of cardiac apoptosis in vivo. Magn Reson Med 2011;66:1152-1162.

Drory Y, Turetz Y, Hiss Y et al. Sudden unexpected death in persons less than 40 years of age. Am J Cardiol 1991;68:1388-1392.

Dweck MR, Abgral R, Trivieri MG et al. Hybrid magnetic resonance imaging and positron emission tomography with fluorodeoxyglucose to diagnose active cardiac sarcoidosis. JACC Cardiovasc Imaging 2017 DOI:10.1016/jcmg.2017.02.021.

Eigenbrod T, Park J-H, Harder J et al. Cutting edge:critical role for mesothelial cells in necrosis-induced inflammation through the recognition of IL-1 $\alpha$ released from dying cells. J Immunol 2008;181:8194-8198.

Entman ML, Smith CW. Postreperfusion inflammation: a model for reaction to injury in cardiovascular disease. Cardiovasc Res 1994;28(9):1301-1311.

Esfandiarei M, McManus BM. Molecular bioogy and pathogenesis of viral myocarditis. Annu Rev Pathol. 2008;3:127.

Feldman AM, McNamara D. Myocarditis. N Engl J Med 2000;343(19):1388-1398.

Fenoglio JJ Jr, McAllister HA Jr, Mullick FG. Drug related myocarditis. I. Hypersensitivity Myocarditis. Hum Pathol. 1981; 12(10):900.

Frangogiannis NG, Youker KA, Rossen RD et al. Cytokines and the microcirculation in ischemia and reperfusion. J Mol Cell Cardiol 1998;30(12):2567-2576.

Frangogiannis NG, Smith CW, Entman ML. The inflammatory response in myocardial infarction. Cardiovasc Res 2002;53(1):31-47.

Friedrich MG, Sechtem U, Schulz-Menger J et al. Cardiovascular magnetic resonance in myocarditis. A JACC White Paper. J Am Coll Cardiol 2009;53(17): $1475-1487$.

Gellissen J, Axmann C, Prescher A, Bohndorf K, Lodemann KP. Extra- and intracellular accumulation of ultrasmall superparamagnetic iron oxides (USPIO) in experimentally induced abscesses of the peripheral soft tissues and their effects on magnetic resonance imaging. Magn Reson Imaging 1999;17(4):557-567.

Giri S, Chung Y-C, Merchant A et al. T2 quantification for improved detection of myocardial edema. J Cardiovasc Magn Reson 2009;11:56.

Go AS, Mozaffarian D, Roger VL et al. Heart disease and stroke statistics - 2014 update: a report from the American Heart Association. Circulation 2014;129:e28-92.

Guimaraes AR, Siqueira L, Uppal R et al. T2 relaxation time is related to liver fibrosis severity. Quant Imaging Med Surg 2016;6:103-114. 
Guo Y, Chen W, Wang W et al. Simultaneous diagnosis and gene therapy of immuno-rejection in rat allogeneic heart transplantation model using a T-celltargeted theranostic nanosystem. ACS Nano 2012;6(12):10646-10657

Hahn PF, Stark DD, Lewis JM et al. First clinical trial of a new superparamagnetic iron oxide for use as an oral gastrointestinal contrast agent in MR imaging. Radiology 1990;175:695-700.

Han S, Xing W, Lu H, Han H. Early diagnostic method for sepsis based on neutrophil MR imaging. Radiol Infect Dis 2015;2(1):33-39.

He T, Gatehouse PD, Kirk P et al. Black-blood T2* technique for myocardial iron measurement in thalassemia. J Magn Reson Imaging 2007;25:1205-1209.

Heesakkers RAM, Jager GJ, Hövels AM et al. Prostate cancer: detection of lymph node metastases outside the routine surgical area with ferumoxtran-10-enhanced MR imaging. Radiology 2009;251(2):408-414.

Hertz MI, Aurora P, Christie JD et al. Scientific Registry of the International Society for Heart and Lung Transplantation: Introduction to the 2009 Annual Reports. J Heart Lung Transplant 2009;28(10):989-992.

Hirasawa K, Tsutsui S, Takeda M, Mizutani M, Itagaki S, Doi K. Depletion of Mac1-positive macrophages protects DBA/2 mice from encephalomyocarditis virusinduced myocarditis and diabetes. J Gen Virol 1996;77(4):737-741.

Ho V, Reddy GP. Cardiovascular imaging. Elsevier Health Sciences 2010.

Howarth SP, Tang TY, Graves MJ et al. Ann Surg Innov Res 2007;1(1):4.

Hyafil F, Laissy J-P, Mazighi M et al. Ferumoxtran-10-enhanced MRI of the hypercholesterolemic rabbit aorta: relationship between signal loss and macrophage infiltration. Arterioscler Thromb Vasc Biol 2006;26(1):176-181.

Ishii D, Scheck AD, Baba S et al. Role of TNF $\alpha$ in early chemokine production and leukocyte infiltration into heart allografts. Am J Transplant. 2010;10:59-68.

Jaquenod De Giusti C, Ure AE, Rivadeneyra L, Schattner M, Gomez RM. Macrophages and galectin 3 play critical roles in CVB3-induced murine acute myocarditis and chronic fibrosis. J Mol Cell Cardiol 2015;85:58-70.

Johansson LO, Nolan MM, Taniuchi M, Fischer SE, Wickline SA, Lorenz CH. Highresolution magnetic resonance coronary angiography of the entire heart using a new blood-pool agent, NC100150 injection: comparison with invasive x-ray angiography in pigs. J Cardiovasc Magn Reson 1999;1(2):139-143.

Johansson L, Johnsson C, Penno E, Bjornerud A, Ahlstrom H. Acute cardiac transplant rejection: detection and grading with MR Imaging with a blood pool contrast agent experimental study in the rat. Radiology 2002;225:97-103. 
Kaim AH, Wischer $\mathrm{T}$, O'Reilly $\mathrm{T}$ et al. MR imaging with ultrasmall superparamagnetic iron oxide particles in experimental soft-tissue infections in rats. Radiology 2002;225:808-814.

Kandolin R, Lehtonen J, Kupari M. Cardiac sarcoidosis and giant cell myocarditis as causes of atrioventricular block in young and middle-aged adults. Circ Arrhythm Electrophysiol 2011;4:303-309.

Kanno S, Wu Y-JL, Lee PC et al. Macrophage accumulation associated with rat cardiac allograft rejection detected by magnetic resonance imaging with ultrasmall superparamagnetic iron oxide particles. Circulation 2001;104:934-938.

Kellman P, Aletras AH, Mancini C et al. T2-prepared SSFP improves diagnostic confidence in edema imaging in acute myocardial infarction compared to turbo spin echo. Magn Reson Med 2007;57:891-897.

Kirk P, He T, Anderson LJ et al. International reproducibility of single breathhold T2* MR for cardiac and liver iron assessment among five thalassemia centers. J Magn Reson Imaging 2010;32:315-319.

Klein C, Schalla S, Schnackenburg B et al. Improvement of image quality of noninvasive coronary artery imaging with magnetic resonance by the use of the intravascular contrast agent Clariscan (NC100150 injection) in patients with coronary artery disease. J Magn Reson Imaging 2003;17(6):656-662.

Kooi ME. Accumulation of ultrasmall superparamagnetic particles of iron oxide in human atherosclerotic plaques can be detected by in vivo magnetic resonance imaging. Circulation 2003;107(19):2453-2458.

Kraitchman DL. In vivo magnetic resonance imaging of mesenchymal stem cells in myocardial infarction. Circulation 2003;107:2290-2293.

Kresse M, Wagner S, Thode K, Dinkelborg L. MR plaque imaging using superparamagnetic iron oxide particles. Proc ISMRM 1998.

Kroft LJM, Doornbos J, van der Geest RJ, van der Laarse A, van der Meulen H, de Roos A. Ultrasmall superparamagnetic particles of iron oxide (USPIO) MR imaging of infarcted myocardium in pigs. Magn Reson Imaging 1998;16:755-763.

Krombach GA, Wendland MF, Higgins CB, Saeed M. MR imaging of spatial extent of microvascular injury in reperfused ischemically injured rat myocardium: value of blood pool ultrasmall superparamagnetic particles of iron oxide. Radiology 2002;225(2):479-486.

Küstermann E, Roell W, Breitbach $\mathrm{M}$ et al. Stem cell implantation in ischemic mouse heart: a high-resolution magnetic resonance imaging investigation. NMR Biomed 2005;18:362-370.

La Rosa DF, Rahman AH, Turka LA. The innate immune system in allograft rejection and tolerance. J Immunol 2007; 178:7503-7509. 
Lee WW, Marinelli B, van der Laan AM et al. PET/MRI of inflammation in myocardial infarction. J Am Coll Cardiol 2012;59:153-163.

Li K, Xu W, Guo Q et al. Differential macrophage polarization in male and female BALB/c mice infected with coxsackievirus B3 defines susceptibility to viral myocarditis. Circ Res 2009;105:353-364.

Lu M, Zhao S, Liu Q et al. Transplantation with autologous mesenchymal stem cells after acute myocardial infarction evaluated by magnetic resonance imaging: an experimental study. $J$ Thorac Imaging 2012;27:125-135.

Luetkens JA, Homsi R, Sprinkart AM et al. Incremental value of quantitative CMR including parametric mapping for the diagnosis of acute myocarditis. Eur Heart $J$ Cardiovasc Imaging 2015; 17(2):154-161.

Ma G-S, Qi C-M, Liu N-F et al. Efficiently tracking of stem cells in vivo using different kinds of superparamagnetic iron oxide in swine with myocardial infarction. Chin Med J 2011;124(8):1199-1204.

Maekawa Y, Anzai T, Yoshikawa T et al. Prognostic significance of peripheral monocytosis after reperfused acute myocardial infarction:a possible role for left ventricular remodeling. J Am Coll Cardiol 2002;39(2):241-246.

Magnani JW, Dec GW. Myocarditis: current trends in diagnosis and treatment. Circulation 2006;113:876-890.

Mahmoudi M, Simchi A, Imani M, Milani AS, Stroeve P. An in vitro study of bare and poly(ethylene glycol)-co-fumarate-coated superparamagnetic iron oxide nanoparticles: a new toxicity identification procedure. Nanotechnology 2009;20(22):225104.

Mahmoudi M, Simchi A, Imani $\mathrm{M}$ et al. A new approach for the in vitro identification of the cytotoxicity of superparamagnetic iron oxide nanoparticles. Colloids and Surfaces B: Biointerfaces 2010;75(1):300-309.

Mandeville JB, Moore J, Chesler DA, Garrido L, Weissleder R, Weisskoff RM. Dynamic liver imaging with iron oxide agents: effects of size and biodistribution on contrast. Magn Reson Med 1997;37(6):885-890.

Mannon RB. Macrophages: contributors to allograft dysfunction, repair, or innocent bystanders? Curr Opin Organ Transplant 2012;17:20-25.

Marie PY, Carteaux JP, Escanye JM et al. Detection and prediction of acute heart transplant rejection with the myocardial $\mathrm{T} 2$ determination provided by a black-blood magnetic resonance imaging sequence. J Heart Lung Transplant 2001;20:193-194.

Mathiasen AB, Hansen L, Friis T, Thomsen C, Bhakoo K, Kastrup J. Optimal labeling dose, labeling time, and magnetic resonance imaging detection limits of ultrasmall superparamagnetic iron-xxide nanoparticle labeled mesenchymal stromal cells. Stem Cells Int 2013;2013:1-10. 
McAteer MA, Akhtar AM, Zur Muhlen von C, Choudhury RP. An approach to molecular imaging of atherosclerosis, thrombosis, and vascular inflammation using microparticles of iron oxide. Atherosclerosis 2010;209:18-27.

McBride OMB, Berry C, Burns $\mathrm{P}$ et al. MRI using ultrasmall superparamagnetic particles of iron oxide in patients under surveillance for abdominal aortic aneurysms to predict rupture or surgical repair: MRI for abdominal aortic aneurysms to predict rupture or surgery-the MA(3)RS study. Open Heart. 2015;2(1):e000190-0.

McLachlan SJ, Morris MR, Lucas MA et al. Phase I clinical evaluation of a new iron oxide MR contrast agent. J Magn Reson Imaging 1994;4(3):301-307.

Miller CA, Naish JH, Shaw SM et al. Multiparametric cardiovascular magnetic resonance surveillance of acute cardiac allograft rejection and characterization of transplantation-associated myocardial injury: a pilot study. J Cardiovasc Magn Reson 2014;16(1):52.

Mitchell DN, Scadding JG, Heard BE, Hinson KF. Sarcoidosis: histopathological definition and clinical diagnosis. J Clin Pathol 1977;30:395-408.

Moon H, Park HE, Kang $\mathbf{J}$ et al. Noninvasive assessment of myocardial inflammation by cardiovascular magnetic resonance in a rat model of experimental autoimmune myocarditis. Circulation 2012;125(21):2603-2612.

Moore A, Weissleder R, Bogdanov A. Uptake of dextran-coated monocrystalline iron oxides in tumor cells and macrophages. J Magn Reson Imaging 1997;7(6): $1140-1145$.

Moreau A, Varney E, Anegon I, Cuturi M-C. Effector Mechanisms of Rejection. Cold Spring Harb Perspect Med. 2013;3(11):a015461

Müller K, Skepper JN, Posfai M et al. Effect of ultrasmall superparamagnetic iron oxide nanoparticles (Ferumoxtran-10) on human monocyte-macrophages in vitro. Biomaterials 2007;28(9):1629-1642.

Nahrendorf M, Swirski FK, Aikawa E et al. The healing myocardium sequentially mobilizes two monocyte subsets with divergent and complementary functions. $J$ Exp Med 2007;204:3037-3047.

Nahrendorf M, Pittet MJ, Swirski FK. Monocytes: Protagonists of infarct inflammation and repair after myocardial infarction. Circulation 2010;121(22): $2437-2445$.

Nilsson JC. Sustained postinfarction myocardial oedema in humans visualised by magnetic resonance imaging. Heart 2001;85:639-642.

Nishii T, Kono AK, Shigeru $\mathrm{M}$ et al. Cardiovascular magnetic resonance T2 mapping can detect myocardial edema in idiopathic dilated cardiomyopathy. Int $J$ Cardiovasc Imaging 2014;30(Suppl 1):65-72. 
Paetsch I, Thiele $\mathrm{H}$, Schnackenburg B et al. Improved functional cardiac MR imaging using the intravascular contrast agent CLARISCAN. Int $J$ Cardiovasc Imaging 2003;19(4):337-343.

Panizzi P, Swirski FK, Figueiredo JL et al. Impaired infarct healing in atherosclerotic mice with Ly-6C. J Am Coll Cardiol 2010;55(15):1629-1638.

Penno E, Johnsson C, Johansson L, Ahlström H. Comparison of ultrasmall superparamagnetic iron oxide particles and low molecular weight contrast agents to detect rejecting transplanted hearts with magnetic resonance imaging. Invest Radiol 2005;40:648-654.

Penno E, Johnsson C, Johansson L, Ahlstrom H. Macrophage uptake of ultra-small iron oxide particles for magnetic resonance imaging in experimental acute cardiac transplant rejection. Informa UK Ltd UK 2009;47(3):264-271.

Richards JMJ, Semple SI, MacGillivray TJ et al. Abdominal aortic aneurysm growth predicted by uptake of ultrasmall superparamagnetic particles of iron oxide: A pilot study. Circ Cardiovasc Imaging 2011;4:274-281.

Roberts WC, McAllister HA, Ferrans VJ. Sarcoidosis of the heart. A clinicopathologic study of 35 necropsy patients (group 1) and review of 78 previously described necropsy patients (group 11). Am J Med 1977;63:86-108.

Rogers JM, Lewis J, Josephson L: Visualization of superior mesenteric lymph nodes by the combined oral and intravenous administration of the ultrasmall superparamagnetic iron oxide, AMI-227. Magn Reson Imaging 1994;12:1161-1165.

Ros PR, Freeny PC, Harms SE et al. Hepatic MR imaging with ferumoxides: a multicenter clinical trial of the safety and efficacy in the detection of focal hepatic lesions. Radiology 1995;196:481-488.

Ruehm SG, Corot C, Vogt P, Kolb S, Debatin JF. Magnetic resonance imaging of atherosclerotic plaque with ultrasmall superparamagnetic particles of iron oxide in hyperlipidemic rabbits. Circulation 2001;103:415-422.

Sadat U, Taviani V, Patterson AJ et al. Ultrasmall superparamagnetic iron oxideenhanced magnetic resonance imaging of abdominal aortic aneurysms-A feasibility study. Eur J Vasc Endovasc Surg 2011;41(2):167-174.

Sagar S, Liu PP, Cooper LT. Myocarditis. Lancet 2012;379:738-747.

Saini S, Stark DD, Wittenberg J, Brady TJ, Ferrucci JT: Ferrite particles: a superparamagnetic MR contrast agent for the reticuloendothelial system. Radiology 1987;162:211-216.

Sandstede JJ, Pabst T, Wacker C et al. Breath-hold 3D MR coronary angiography with a new intravascular contrast agent (feruglose)-first clinical experiences. Magn Reson Imaging 2001;19(2):201-205. 
Sanjay K, Shetty MD, Mukesh G, Harisinghani MD. Magnetic resonance techniques in lymph node imaging. Appl Radiol July 09, 2004.

Schellenberger EA, Högemann D, Josephson L, Weissleder R. Annexin V-CLIO: a nanoparticle for detecting apoptosis by MRI. Acad Radiol 2002;9(Suppl 2):S310-1.

Schmitz SA, Taupitz M, Wagner S, Wolf KJ, Beyersdorff D, Hamm B. Magnetic resonance imaging of atherosclerotic plaques using superparamagnetic iron oxide particles. J Magn Reson Imaging 2001;14(4):355-361.

Schultheiss HP. The significance of autoantibodies against the ADP/ATP carrier for the pathogenesis of myocarditis and dilated cardiomyopathy - clinical and experimental data. Springer Semin Immunopathol. 1989;11(1):15.

Shah S, Xue H, Greiser A et al. Inline myocardial t2* mapping with iterative robust fitting. J Cardiovasc Magn Reson 2011;13:308.

Simon GH, Vopelius-Feldt von J, Wendland MF et al. MRI of arthritis: comparison of ultrasmall superparamagnetic iron oxide vs. Gd-DTPA. J Magn Reson Imaging 2006;23:720-727.

Sosnovik DE, Schellenberger EA, Nahrendorf M et al. Magnetic resonance imaging of cardiomyocyte apoptosis with a novel magneto-optical nanoparticle. Magn Reson Med 2005;54:718-724.

Sosnovik DE, Nahrendorf M, Deliolanis N et al. Fluorescence tomography and magnetic resonance imaging of myocardial macrophage infiltration in infarcted myocardium in vivo. Circulation 2007;115(11):1384-1391.

Sosnovik DE, Garanger E, Aikawa E et al. Molecular MRI of cardiomyocyte apoptosis with simultaneous delayed-enhancement MRI distinguishes apoptotic and ncrotic myocytes in vivo potential for midmyocardial salvage in acute ischemia. Circ Cardiovasc Imaging 2009;2:460-467.

St Pierre TG, Clark PR, Chua-anusorn W et al. Noninvasive measurement and imaging of liver iron concentrations using proton magnetic resonance. Blood 2005;105(2):855-861.

Stewart S, Winters GL, Fishbein MC et al. Revision of the 1990 working formulation for the standardization of nomenclature in the diagnosis of heart rejection. J Heart Lung Transplant 2005;24:1710-1720.

Stillman AE, Wilke N, Jerosch Herold M. Use of an intravascular T1 contrast agent to improve MR cine myocardial-blood pool definition in man. J Magn Reson Imaging 1997;7(4):765-767.

Stirrat CG, Alam SR, MacGillivray TJ et al. Ferumoxytol-enhanced magnetic resonance imaging methodology and normal values at 1.5 and $3 \mathrm{~T}$. $J$ Cardiovasc Magn Reson 2016;18(1):46. 
Stirrat CG, Alam SR, MacGillivray TJ et al. Ferumoxytol-enhanced magnetic resonance imaging assessing inflammation after myocardial infarction. Heart 2017;103:1528-1535.

Tang T, Howarth SPS, Miller SR et al. Assessment of inflammatory burden contralateral to the symptomatic carotid stenosis using high-resolution ultrasmall, superparamagnetic iron oxide-enhanced MRI. Stroke 2006;37:2266-2270.

Tang TY, Howarth SPS, Miller SR et al. Comparison of the inflammatory burden of truly asymptomatic carotid atheroma with atherosclerotic plaques in patients with asymptomatic carotid stenosis undergoing coronary artery bypass grafting: an ultrasmall superparamagnetic iron oxide enhanced magnetic resonance study. Eur $J$ Vasc Endovasc Surg 2008;35(4):392-398.

Tang TY, Howarth SPS, Miller SR et al. The ATHEROMA (Atorvastatin Therapy: Effects on reduction of macrophage activity) study evaluation using ultrasmall superparamagnetic iron oxide-enhanced magnetic resonance imaging in carotid disease. J Am Coll Cardiol 2009;53(22):2039-2050.

Tanner MA, He T, Westwood MA, Firmin DN, Pennell DJ, Thalassemia International Federation Heart $\mathrm{T} 2 *$ Investigators. Multi-center validation of the transferability of the magnetic resonance $\mathrm{T} 2 *$ technique for the quantification of tissue iron. Haematologica 2006;91:1388-1391.

Taylor AM, Panting JR, Keegan $\mathrm{J}$ et al. Use of the intravascular contrast agent NC100150 injection in spin-echo and gradient-echo imaging of the heart. J Cardiovasc Magn Reson 1999a;1(1):23-32.

Taylor AM, Panting JR, Keegan J et al. Safety and preliminary findings with the intravascular contrast agent NC100150 injection for MR coronary angiography. $J$ Magn Reson Imaging 1999b;9:220-227.

Thavendiranathan $\mathrm{P}$, Walls $\mathrm{M}$, Giri $\mathrm{S}$ et al. Improved detection of myocardial involvement in acute inflammatory cardiomyopathies using T2 mapping. Circ Cardiovasc Imaging 2012;5:102-110.

Thorek DLJ, Chen AK, Czupryna J et al. Superparamagnetic iron oxide nanoparticle probes for molecular imaging. Ann Biomed Eng 2006;34:23-38.

Thygesen K, Alpert JS, Jaffe AS et al. Third Universal Definition of Myocardial Infarction. J Am Coll Cardiol 2012;60(16):1581-1598.

Trillaud H, Degrèze P, Mesplède Y, Douws C, Palussière J, Grenier N. Evaluation of experimentally induced renal hypoperfusion using iron oxide particles and fast magnetic resonance imaging. Acad Radiol 1995;2(4):293-299.

Trivedi RA, U-King-Im JM, Graves MJ et al. In vivo detection of macrophages in human carotid atheroma: temporal dependence of ultrasmall superparamagnetic particles of iron oxide-enhanced MRI. Stroke 2004;35:1631-1635. 
Trivedi RA, Mallawarachi C, U-King-Im JM et al. Identifying inflamed carotid plaques using in vivo USPIO-enhanced MR imaging to label plaque macrophages. Arterioscler Thromb Vasc Biol 2006;26: 1601-1606.

Truijers M, Fütterer JJ, Takahashi S, Heesakkers RA, Blankensteijn JD, Barentsz JO. In vivo imaging of the aneurysm wall with MRI and a macrophage-specific contrast agent. Am J Roentgenol 2009;193(5):W437-W441.

Tsujioka $\mathrm{H}$, Imanishi $\mathrm{T}$, Ikejima $\mathrm{H}$ et al. Impact of heterogeneity of human peripheral blood monocyte subsets on myocardial salvage in patients with primary acute myocardial infarction.. J Am Coll Cardiol 2009;54(2):130-138.

Usman AA, Taimen $\mathrm{K}$, Wasielewski $\mathrm{M}$ et al. Cardiac magnetic resonance $\mathrm{T} 2$ mapping in the monitoring and follow-up of acute cardiac tansplant rejection clinical perspective. Circ Cardiovasc Imaging 2012;5:782-790.

Van Beers BE, Sempoux C, Materne R et al. Biodistribution of ultrasmall iron oxide particles in the rat liver. J Magn Reson Imaging 2001;13:594-599.

Verhaert D, Thavendiranathan P, Giri S et al. Direct T2 quantification of myocardial edema in acute ischemic injury. JACC: Cardiovascular Imaging 2011;4:269-278.

Wang Y-XJ, Hussain SM, Krestin GP. Superparamagnetic iron oxide contrast agents: physicochemical characteristics and applications in MR imaging. Eur Radiol 2001;11:2319-2331.

Wang Y-XJ. Superparamagnetic iron oxide based MRI contrast agents: current status of clinical application. Quant Imaging Med Surg 2011;1(1):35-40.

Wang ZJ, Lian L, Chen Q, Zhao H, Asakura T, Cohen AR. 1/T2 and magnetic susceptibility measurements in a gerbil cardiac iron overload model. Radiology 2005;234(3):749-755.

Weinstein JS, Varallyay CG, Dosa E et al. Superparamagnetic iron oxide nanoparticles: diagnostic magnetic resonance imaging and potential therapeutic applications in neurooncology and central nervous system inflammatory pathologies, a review. J Cereb Blood Flow Metab 2010;30(1):15-35.

Westwood M, Anderson LJ, Firmin DN et al. A single breath-hold multiecho T2* cardiovascular magnetic resonance technique for diagnosis of myocardial iron overload. J Magn Reson Imaging 2003a;18:33-39.

Westwood MA, Anderson LJ, Firmin DN et al. Interscanner reproducibility of cardiovascular magnetic resonance $\mathrm{T} 2 *$ measurements of tissue iron in thalassemia. J Magn Reson Imaging 2003b;18:616-620.

Wood JC. Cardiac iron determines cardiac T2*, T2, and T1 in the gerbil model of iron cardiomyopathy. Circulation 2005; 112(4):535-543. 
Wu YL, Ye Q, Sato K, Foley LM, Hitchens TK, Ho C. Noninvasive evaluation of cardiac allograft rejection by cellular and functional cardiac magnetic resonance. $J C M G ; 2009 ; 2(6): 731-741$.

Wu YL, Ye Q, Eytan DF et al. Magnetic resonance imaging investigation of macrophages in acute cardiac allograft rejection after heart transplantation. Circ Cardiovasc Imaging 2013;6:965-973.

Wunderbaldinger P, Josephson L, Weissleder R. Crosslinked iron oxides (CLIO): a new platform for the development of targeted MR contrast agents. Acad Radiol 2002;9(Suppl 2):S304-306.

Yang Y, Yang Y, Yanasak N, Schumacher A, Hu TCC. Temporal and noninvasive monitoring of inflammatory-cell infiltration to myocardial infarction sites using icrometer-sized iron oxide particles. Magn Reson Med 2010;63(1):33-40.

Yang Y, Liu J, Yang Y, Cho SH, Hu TCC. Assessment of cell infiltration in myocardial infarction: A dose-dependent study using micrometer-sized iron oxide particles. Magn Reson Med 2011;66(5):1353-1361.

Yazaki Y, Isobe M, Hiroe M et al. Prognostic determinants of long-term survival in Japanese patients with cardiac sarcoidosis treated with prednisone. Am J Cardiol 2001;88:1006-1010.

Yilmaz A, Dengler MA, van der Kuip $\mathrm{H}$ et al. Imaging of myocardial infarction using ultrasmall superparamagnetic iron oxide nanoparticles: a human study using a multi-parametric cardiovascular magnetic resonance imaging approach. Eur Heart $J$ 2013;34:462-475.

Zimmer C, Weissleder R, O'Connor D, LaPointe L, Brady TJ, Enochs WS. Cerebral iron oxide distribution: in vivo mapping with MR imaging. Radiology 1995; 196(2):521-527. 


\section{Publications}

Stirrat CG, Alam SR, MacGillivray TJ et al. Ferumoxytol-enhanced magnetic resonance imaging in acute myocarditis. Accepted by Heart 2017, awaiting publication.

Stirrat CG, Alam SR, MacGillivray TJ et al. Ferumoxytol-enhanced magnetic resonance imaging assessing inflammation after myocardial infarction. Heart 2017; 103:1528-1535.

Stirrat CG, Alam SR, MacGillivray TJ et al. Ferumoxytol-enhanced magnetic resonance imaging methodology and normal values at 1.5 and $3 \mathrm{~T}$. $J$ Cardiovasc Magn Reson 2016;18:46.

Stirrat CG, Vesey AT, McBride OMB et al. Ultra-small superparamagnetic particles of iron oxide in magnetic resonance imaging of cardiovascular disease. J Vasc Diagnostics 2014;2:99-112.

Stirrat CG, Newby DE, Robson JMJ, Jansen MA. The use of superparamagnetic iron oxide nanoparticles to assess cardiac inflammation. Curr Cardiovasc Imaging Rep 2014;7:9263. DOI 10.1007/s12410-014-9263-3. 


\title{
The Use of Superparamagnetic Iron Oxide Nanoparticles to Assess Cardiac Inflammation
}

\author{
Colin G. Stirrat • David E. Newby • \\ Jennifer M. J. Robson • Maurits A. Jansen
}

(C) Springer Science+Business Media New York 2014

\begin{abstract}
Superparamagnetic iron oxide-based contrast agents enhance and complement in vivo magnetic resonance imaging (MRI) by shortening $\mathrm{T} 2$ and $\mathrm{T} 2 *$ relaxation times. They are able to highlight areas of cellular inflammation, being detected and engulfed by cells of the reticuloendothelial system, and can be targeted to specific cellular processes or subtypes using antibody or ligand labeling. These agents have been used preclinically for the assessment of cardiac transplant rejection, cardiomyocyte apoptosis, myocardial infarction, myocarditis, and stem and endothelial cell imaging, with clinical applications now emerging. We here review recent studies using iron oxide particles to image cardiac inflammation, and highlight the potential of these agents for future clinical and research applications.
\end{abstract}

Keywords Cardiac · MRI · Iron oxide nanoparticles · Inflammation $\cdot$ Molecular imaging

\section{Introduction}

Overview of Iron Oxide Nanoparticles

In many inflammatory diseases of the myocardium, endomyocardial biopsy is the 'gold standard' diagnostic investigation. However, this does carry the potential for lifethreatening complications [1], and false negative or inconclusive results are not uncommon due to the focal nature and spatial heterogeneity of the underlying inflammatory disease

This article is part of the Topical Collection on Molecular Imaging

C. G. Stirrat $(\triangle) \cdot$ D. E. Newby · J. M. J. Robson · M. A. Jansen British Heart Foundation/University Centre for Cardiovascular Science, University of Edinburgh, Room SU 305 Chancellor's Building, 49 Little France Crescent, Edinburgh EH16 4SB, UK e-mail: colin.stirrat@ed.ac.uk process. Development of a reliable noninvasive imaging technique capable of detecting myocardial inflammation would be extremely beneficial and a major advance. This could provide a diagnosis in patients displaying early signs suggestive of myocardial inflammation or with subclinical disease, creating an opportunity to prevent the disease process from become established or limit its progression. Furthermore, this could provide a surrogate measure of treatment response and facilitate serial disease monitoring.

Iron oxide nanoparticles can be used for molecular and cellular imaging [2], and thereby enhance the sensitivity of magnetic resonance imaging (MRI) to detect and characterize various pathologic processes [3]. They rely on the principle that accumulation of superparamagnetic iron oxide particles within tissues causes local magnetic field inhomogeneities that shorten $\mathrm{T} 2$ and $\mathrm{T} 2 *$ relaxation times. They generally consist of an iron oxide core surrounded by a carbohydrate or polymer coat that prevents aggregation and oxidation of the iron, and provides a surface for conjugation of drug molecules and targeting ligands. A range of particles exists and they vary in size and coating. These 2 properties of iron nanoparticles are the key determinants of their biodistribution, magnetic behavior and, thus, the potential applications of the particle $[4,5]$.

Iron oxide particles can be categorized according to their size (Table 1): nano-sized very small (VSPIO, $<20 \mathrm{~nm}$ in diameter), ultrasmall (USPIO, 20-50 nm in diameter) and small (SPIO, 60-250 $\mathrm{nm}$ in diameter) superparamagnetic particles of iron oxide, and micrometer-sized (MPIO, 1-8 $\mu \mathrm{m}$ in diameter) particles of iron oxide. The 'superparamagnetic' particles become magnetic within a strong external magnetic field such as exists within an MRI scanner, but do not retain their magnetism once removed from the field.

Iron oxide particles were initially used as contrast agents for imaging of the gastrointestinal tract [6], the Kupffer cells of the liver and spleen [7], and lymph nodes [8], as well as a 
Table 1 Summary of superparamagnetic particles of iron oxide

\begin{tabular}{|c|c|c|c|c|c|c|}
\hline Particle Name & Particle Size (diameter) & Agent Name & Coating & Clinical/ Preclinical & Application & Reference \\
\hline \multirow[t]{4}{*}{ USPIO } & \multirow[t]{4}{*}{$20-50 \mathrm{~nm}$} & $\mathrm{n} / \mathrm{a}$ & Dextran & Preclinical & Allograft rejection & {$[25]$} \\
\hline & & $\mathrm{n} / \mathrm{a}$ & Dextran & Preclinical & Allograft rejection & {$[26]$} \\
\hline & & $\mathrm{n} / \mathrm{a}$ & Polymer & Preclinical & Allograft rejection & {$[27 \bullet]$} \\
\hline & & Ferumoxytol & Dextran & Clinical & Myocardial Infarction & {$[35 \bullet \bullet, 36 \bullet \bullet$} \\
\hline CLIO & $30-50 \mathrm{~nm}$ & $\mathrm{n} / \mathrm{a}$ & Dextran & Preclinical & Apoptosis & {$[32,33]$} \\
\hline \multirow[t]{5}{*}{ SPIO } & \multirow[t]{5}{*}{$60-250 \mathrm{~nm}$} & Ferumoxides & Dextran & Preclinical & Apoptosis & [29] \\
\hline & & $\mathrm{n} / \mathrm{a}$ & PEG/silica & Preclinical & Myocarditis & [39] \\
\hline & & Ferumoxides & Dextran & Preclinical & Stem Cells & [40] \\
\hline & & Ferucarbotran & Carbodextran & Preclinical & Stem Cells & [41] \\
\hline & & $\mathrm{n} / \mathrm{a}$ & Polysaccharide & Preclinical & Stem cells & {$[42 \cdot]$} \\
\hline \multirow[t]{3}{*}{ MPIO } & \multirow[t]{3}{*}{$1-8 \mu \mathrm{m}$} & ME03F & Polymer & Preclinical & Myocardial Infarction & {$[18,34]$} \\
\hline & & MyOne & $\mathrm{n} / \mathrm{a}$ & Preclinical & VCAM-1 & [37] \\
\hline & & $\mathrm{n} / \mathrm{a}$ & $\mathrm{n} / \mathrm{a}$ & Preclinical & VCAM-1 & {$[43]$} \\
\hline
\end{tabular}

blood-pool contrast agent for the evaluation of cerebral, myocardial, and renal perfusion [9-11]. Here, we focus on its application to image cardiac inflammation with an emphasis on recent findings, and comment on future applications.

\section{USPIO and VSPIO}

The VSPIO particle is the smallest of the iron particles and the USPIO particle is only slightly larger; hence, these agents are often classified together as USPIO. Their clinical application makes use of their ability to escape immediate phagocytosis by cells of the reticuloendothelial system, conferring a considerably longer half-life within the blood pool (up to 24 hours [12]) compared with larger particles. Both VSPIO and USPIO have been used successfully as contrast agents for MR coronary angiography $[13,14]$ because they have a longer blood pool circulating time compared with standard MR angiographic agents such as Gadolinium. Although USPIO remain predominantly within the blood pool, they are small enough to migrate passively across the endothelium [2] especially in regions of the vasculature where there is loss of endothelial integrity. Once present within tissues, resident macrophages with enhanced phagocytic activity engulf and concentrate these nanoparticles. This colocalization and concentration of nanoparticles enhances their superparamagnetic effects markedly shortening the $\mathrm{T} 2 *$ value and creating local signal deficits that appear dark on T2- and T2*-weighted images (Fig. 1). It is these magnetic effects of USPIO that allow their use to detect capillary leakiness and tissue-resident inflammatory cell activity, thereby highlighting areas of tissue inflammation.

\section{SPIO}

SPIOs are larger than USPIOs, and as such are more rapidly phagocytosed by cells of the reticuloendothelial system in the liver, spleen, bone marrow, and lymph nodes (Fig. 2). They are rapidly cleared from the blood pool after injection having a short blood pool half-life of less than 15 minutes [15] in rats and around 2 hours in humans [16]. After intracellular uptake, SPIOs are degraded into a nonsuperparamagnetic form of iron that becomes incorporated into the body's iron stores [2]. The coating of the particle also has to be metabolized, and coating with dextran confers the advantage that it is biodegradable and excreted in urine and feces [17].

\section{MPIO}

The largest of the iron particles discussed, MPIOs, are often used for molecular imaging of endovascular targets. As a result of their large size MPIOs have a very short blood pool half-life of $<1$ minute in mice [18] and $<2$ minutes in rats [19]. Following intravenous administration phagocytic cells within the blood pool and the lymphoreticular system identify them rapidly. However, until they are cleared, they are less susceptible than smaller particles to migrate across endovascular borders or be incorporated into endothelial cells, and are confined to the intravascular compartment [20]. For this reason, MPIOs are often used to provide a platform for quantitative targeted molecular imaging of vascular endothelial targets. By labeling cells ex vivo with MPIO, single cell detection has been shown in vivo [21]. Importantly, MPIO contain significantly more iron oxide than USPIO or SPIO and consequently fewer MPIO are required for detection. Indeed, they create such an effect on local magnetic field homogeneity that the spatial extent of the change in signal intensity on T2- and $\mathrm{T} 2 *$-weighted imaging can be up to 50 times its own physical diameter. This so-called "blooming effect" markedly enhances sensitivity such that MPIOs can detect even sparse endothelial molecular targets [22], but spatial information may be compromised. 
Fig. 1 Patient imaged 5 days after an anterior myocardial infarct with $\mathrm{T} 2 *$-weighted magnetic resonance imaging. Images show $\mathrm{T}^{*} *$ maps of baseline pre-iron scan (left), and 24 hours following ferumoxytol (USPIO) administration (right). Iron uptake is seen in the dark myocardial regions in the region of the anterior infarct (arrow)
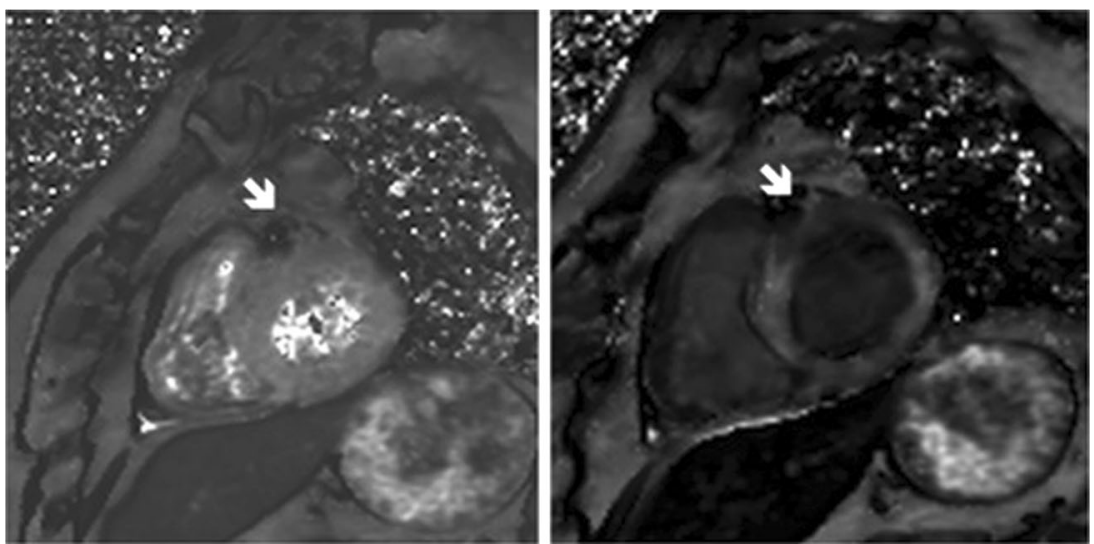

\section{Imaging Using Iron Particles}

Early preclinical imaging work took advantage of the ability of inflammatory cells of the reticuloendothelial system to engulf iron particles enabling the iron particles to act as a tracer localizing to areas of inflammation. Cardiac pathologies examined include cardiac transplantation, cardiomyocyte apoptosis, myocardial infarction (MI), and myocarditis. The use of iron particles has also been employed to examine cellular apoptosis and tracking of stem cells. Histologic validation is important and tissue can be stained with Prussian Blue or Perl's stain for iron (Fig. 3) and colocalization can be confirmed if the same tissue is subsequently stained for inflammatory cells such as macrophages (CD68 immunohistochemistry).

\section{Cardiac Transplant Rejection}

Cardiac transplantation is a major treatment option for endstage cardiac disease, but is challenged by the development of transplant rejection in $20 \%-30 \%$ of recipients in the first year

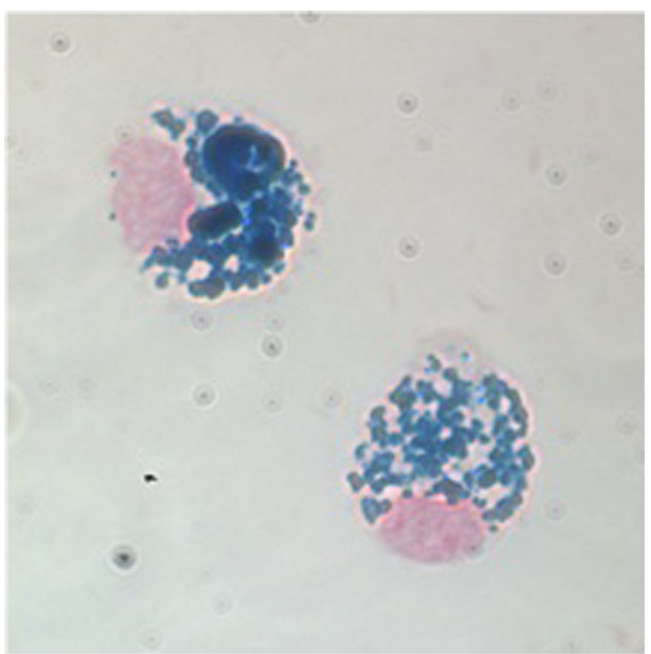

Fig. 2 Perl's iron stain reveals aggregates of SPIO within the cytoplasm of human mononuclear cells labeled with SPIO ex vivo
[23]. This causes significant morbidity and mortality. Transplant rejection can occur at any stage after transplantation and is notoriously difficult to diagnose noninvasively. It is characterized histologically using the International Society for Heart and Lung Transplantation (ISHLT) classification system and graded into 1 of 3 groups depending on the presence of interstitial and perivascular mononuclear cell infiltrates, myocyte damage, edema, hemorrhage, or vasculitis [24].

Endomyocardial biopsy is undertaken routinely for rejection surveillance following transplantation, although this procedure can be associated with serious complications that include damage to the underlying cardiac structure, bleeding, arrhythmia, and death [1]. Kanno et al. [25] detected organ rejection using MRI and dextran-coated USPIO in rats with evidence of cardiac allograft rejection. Histopathology not only confirmed active rejection, but also confirmed UPSIO uptake was confined to tissue-resident macrophages that accumulated within the rejecting cardiac allograft. Interestingly, in rats that had more prolonged immunosuppression, they also

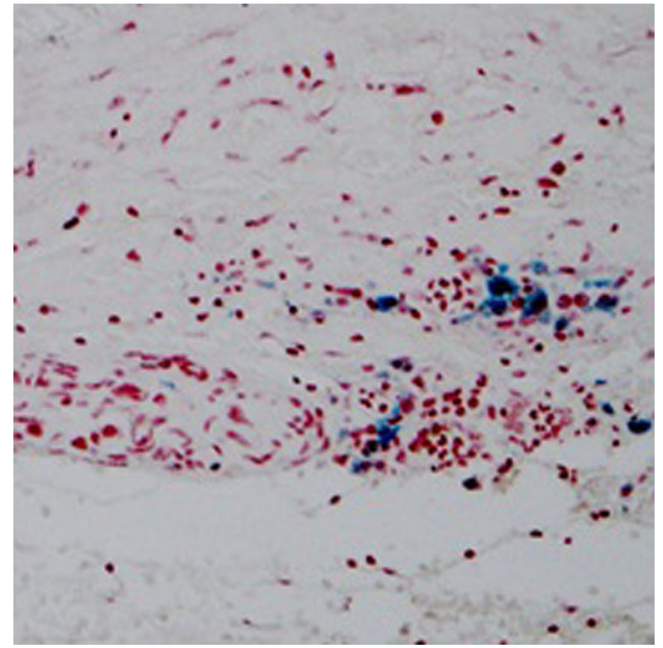

Fig. 3 Perl's iron stain of the aortic wall from a patient with an abdominal aortic aneurysm. Tissue sample was acquired during operative repair 4 days following the administration of USPIO. USPIO is seen to have accumulated within macrophages in an inflamed region of the tunica media 
observed a decline in the rate of signal intensity reduction, suggesting less inflammation and indicating that successful treatment response could also be monitored by this method.

Wu et al. [26] studied a rat model of heart-lung transplant rejection involving the implantation of an additional intraabdominal heart and lung allowing the native heart to support life despite severe rejection in the transplanted organs. USPIO was administered 1 week following transplantation. One day after USPIO administration, hypointense areas appeared on T2*-weighted images of allograft hearts that were not present within the control native hearts and immediate histology after graft explantation revealed accumulation of iron particles mostly within macrophages.

Targeting of various pathophysiological processes of interest becomes possible when contrast agents, including iron oxide particles, are conjugated to a specific antibody or peptide [12]. Recent work by Guo et al. [27•] targeted T cells that play a central role in acute rejection in most cardiac allografts. They built on work performed using ligand targeting of cells to employ a "theranostic" strategy, combining diagnostic imaging with administration of therapy within the 1 system. They targeted $\mathrm{T}$ cells in the endocardium of abdominally implanted rejecting rat hearts using a CD-3 antibody-targeted multifunctional polymeric nanocarrier. This polyplex nanoparticle was further conjugated to a therapy gene (pDNADGKalpha) enabling simultaneous imaging of primary T cells expressing CD-3 and achieving their immune modulation via gene therapy.

\section{Cardiomyocyte Apoptosis}

Apoptosis is the process of programmed cell death in an organism and in the myocardium confers a survival advantage to the organism over necrosis, which induces inflammation and leads to scarring. During apoptosis the phospholipid phosphatidylserine is exteriorized and displayed on the cell surface. Annexin V is an endogenous protein that binds with high affinity to phosphatidylserine. Narula et al. [28] used ${ }^{99 \mathrm{~m}}$ Technetium-labelled Annexin $\mathrm{V}$ to image apoptosis in cardiac allograft rejection in humans. Myocardial ${ }^{99 \mathrm{~m}}$ Technetium-labelled Annexin V binding was observed in 5 out of the 18 human patients with cardiac allografts. All 5 of these patients displayed at least moderate rejection histologically and demonstrated caspase-3 staining, a marker for apoptosis. In contrast, none of the 13 patients with no annexin uptake displayed histologic signs of necrosis.

Dash et al. [29] developed a molecular MRI probe using SPIO conjugated to recombinant human Annexin V (ANXSPIO). Using T2*-weighted MRI, they were able to detect doxorubicin-induced early apoptosis in mouse hearts in vivo. There was a spatial correlation between areas of signal attenuation on $\mathrm{T} 2 *$-weighted images and histologic evidence of iron accumulation and apoptosis (caspase activity), although myocardial structure was normal suggesting only early apoptosis. They were also able to detect apoptosis in cultured ex vivo neonatal rat ventricular myocytes much earlier than TUNEL staining: the latter detects DNA fragmentation and is a late terminal event in the apoptotic sequence. Perhaps more importantly, they were able to use ANX-SPIO to demonstrate that the alpha-1 adrenoreceptor agonist, A61603, could prevent doxorubicin-induced cardiomyopathy. They documented early apoptosis through increased caspase activation and reduction in myocardial T2* value that could be eliminated by A61603 treatment.

The development of cross-linked particles of iron oxide (CLIO) [30] was exploited for targeted imaging of apoptosis again conjugated to annexin V [31]. Cross-linking and amination of the USPIO dextran chains encourages circumferential adherence of the dextran coat to the iron nanoparticle providing chemical stability and improved conjugation [30]. This allowed Sosnovik et al. [32] to develop a CLIO particle conjugated not only to annexin $\mathrm{V}$, but also to cyanine 5.5 dye to allow co-identification with fluorescence microscopy and T2*-weighted MRI. In a murine model of ischemia reperfusion injury, a reduction in signal intensity on $\mathrm{T} 2 *$-weighted imaging was observed with AnxCLIO-Cy5.5 but not with unlabeled CLIO-Cy5.5. The area of signal loss correlated with the zone of contractile dysfunction suggesting uptake of AnxCLIO-Cy5.5 was within the injured and apoptotic myocardium. This was further confirmed by near infra-red fluorescence where increased fluorescence intensity co-localized to regions of reduced signal intensity on MRI.

More recently, Sosnovik et al. employed a similar concept in developing a novel dual-contrast molecular MRI technique to image both cardiomyocyte apoptosis and necrosis in mice with ischemia reperfusion injury in vivo [33]. They used a similar protocol to identify apoptosis and followed this with injection of a novel magnetofluorescent gadolinium chelate (Gd-DTPA-NBD), with subsequent gadolinium delayed enhancement MRI. Interestingly, they found that only $21 \%$ of apoptotic myocardium (accumulation of AnxCLIO5.5) had evidence of necrosis (defined by delayed enhancement with Gd-DTPA-NBD). This was confirmed on fluorescence microscopy and immunohistochemistry revealing the presence of large numbers of apoptotic, but potentially viable, cardiomyocytes in the 'at-risk' area (AnxCLIO-Cy5.5 positive, Gd-DTPA-NBD negative areas). The authors concluded by remarking that strategies to salvage apoptotic, but not yet necrotic, myocardium should be explored now that these areas could be successfully imaged.

\section{Myocardial Infarction}

Yang et al. [18] imaged inflammatory cell infiltration in a murine left anterior descending coronary artery ligation model of myocardial infarction. Micrometer-sized particles of iron 
oxide (MPIO) were administered and $\mathrm{T} 2 *$ signal attenuation was seen in the infarct zone of these mice when compared with control groups of mice that (I) were administered MPIO and had a "sham' operation, or (II) were subjected to MI but did not receive MPIO. Unsurprisingly, they noted increased signal attenuation in the infarct area, illustrating MPIO accumulation secondary to increased inflammatory cell infiltration. This correlated functionally with a reduction in left ventricular ejection fraction.

In a subsequent study by the same group [34], they explored the dose and timing of MPIO administration and MRI scanning. The investigators concluded that an optimal temporal scanning window of 7-14 days post-MI, and an optimal dose of $9.1-14.5 \mu \mathrm{g}$ of iron per gram bodyweight to image myocardial inflammation.

Alam et al. [35••] used USPIO to image inflammation dynamics following acute myocardial infarction (MI). Within 48 hours following acute MI, patients received an infusion of ferumoxytol (USPIO) and underwent MRI scanning at baseline (pre-USPIO) and for 3 days. The infarct zone was defined using a late gadolinium enhancement (LGE) scan. Following USPIO administration, there was a significant increase in $\mathrm{R} 2 *(\mathrm{R} 2 *=1 / \mathrm{T} 2 *)$ in the infarct zone, with smaller changes observed in the peri-infarct zone (surrounding LGE) and even in remote myocardium when compared with control tissue (skeletal muscle). The authors suggested that this was a reflection of peri-infarct and remote myocardial inflammation, although there were no histologic data to confirm this. Notably, the R2* value was greatest 24 hours following USPIO (ferumoxytol) infusion, and had declined by 48 hours suggesting that 24 hours postinfusion is the most sensitive imaging time point in this group of patients.

In a similar study, Yilmaz et al. [36••] also detected a reduction in $\mathrm{T}^{*}$ value in the infarct as well as in the periinfarct region, the remote myocardium and the lymphoreticular system. In imaging the first 3 patients at 6 , 24, 48, and 96 hours after ferumoxytol administration, they attempted to find the optimal time to characterize inflammation kinetics within the myocardium. From these data, they suggest that signal attenuation was maximal at 24 hours but that the best combination of T2-weighted (hypoenhancement seen) and $\mathrm{T} 2 *$-weighted images were obtained at 48 hours.

Unfortunately, neither Yilmaz nor Alam had access to histologic samples. Such data would address the outstanding questions regarding the exact distribution of USPIO within the myocardium, and the potential contribution to the changes in $\mathrm{T} 2 *$ or $\mathrm{R} 2 *$ of extracellular iron accumulation within the interstitium, capillary bed, or tissue resident macrophages.

The finding of $\mathrm{T}^{*} *$ signal void in remote myocardium in the above articles is an interesting observation suggesting a pan-myocardial inflammatory response in the context of a smaller, highly localized infarct. This finding is in agreement with Grieve et al. [37] who used a rat model of ischemia- reperfusion injury, and examined endovascular inflammation using a targeted MPIO tracer. They found MPIO signal in the myocardium and pericardium in segments remote from the ischemia-reperfusion injury. In a mouse model of myocardial infarction, remote inflammation after myocardial infarction has also been demonstrated [38]. An increase in inflammatory mediators was found not only within the remote myocardium, but also within renal glomeruli suggesting that inflammation is not only confined to the myocardium, but extends to distal sites and tissues.

\section{Myocarditis}

Transplant rejection and myocardial infarction are not the only inflammatory myocardial processes to be scrutinized by molecular imaging employing iron particles. Like transplant rejection, myocarditis suffers from the lack of a reliable noninvasive diagnostic test, and again patients are subjected to the risk of biopsy procedures that have a lower diagnostic sensitivity than clinicians would wish.

Moon et al. [39] recently examined a rat model of experimental autoimmune myocarditis. They made use of superparamagnetic and fluorescent "magneto-fluorescent nanoparticles" (MNPs) consisting of a SPIO core with a rhodamine isothiocyanate (RITC)-incorporated silica coating of approximately $20 \mathrm{~nm}$ thickness, and polyethylene glycol (PEG) conjugated onto the silica coating. They compared MNP (10 mg Fe/kg) contrast MRI with unenhanced conventional MRI protocols using T2-weighted, T1-weighted and early and late gadolinium enhancement sequences. The degree of inflammation was also assessed histologically. MNPenhanced $\mathrm{T} 2 *$-weighted CMR more accurately detected scattered foci of inflammation within the myocardium of rats with autoimmune myocarditis and improved visualization of small and less-severe myocardial inflammation than conventional CMR imaging. Areas detected by MNP-enhanced T2*weighted CMR correlated with inflammatory foci and macrophage accumulation on histology as well as MNP-derived fluorescence signals on fluorescence microscopy. However, MNP-enhanced MRI ultimately proved unable to detect mild histologic grades of myocardial inflammation, and this may limit its clinical applicability.

\section{Stem Cells}

Current research endeavors are increasingly focusing on stem cell therapy in an attempt to restore function to organs that were previously felt to have no or little regenerative capacity. There is, therefore, a clear need to track stem cells when administered for therapeutic and regenerative purposes so that the distribution and fate of administered cells can be determined. Kraitchman et al. [40] labeled mesenchymal stem cells (MSC) with SPIO in order to detect and track them 
noninvasively using MRI in a swine model of myocardial infarction. Labeling did not appear to affect the viability of the MSCs, and hypointense regions were seen on MRI within infarcted myocardium after intramyocardial SPIO-MSC injection, suggesting uptake of the SPIO-MSC conjugate.

$\mathrm{Lu}$ et al. [41] recently investigated the effects of culprit vessel intracoronary infusion of SPIO-labeled autologous bone marrow-derived MSCs after acute myocardial infarction in pigs. One-week postmyocardial infarction SPIO-labeled MSCs were infused via the culprit coronary vessel and compared with a control infusion of phosphate-buffer saline. The pigs were imaged immediately before and 3 days after cell transplantation. A reduction in myocardial $\mathrm{T} 2 *$ value was observed after cell transplantation in the study group compared with the control group. However, the majority of the infused cells were found in the spleen, liver, and kidney, resulting in a more pronounced reduction in $\mathrm{T} 2 *$ values in those organs. Immunohistochemistry confirmed this to be the SPIO-MSC conjugate, suggesting the majority of the infused cells escaped into the systemic circulation. There was no difference seen between groups in terms of global or regional cardiac function or myocardial viability although the followup in this group was too short to see any functional recovery. The authors acknowledge a weakness of this method is that signal may be "diluted" if the stem cells subsequently proliferate. Furthermore, this cell tracking method could create misleading results. Although not demonstrated by this study, the iron nanoparticle could become detached from the stem cell allowing it to be taken up by another cell, or left behind in the tissue.

In a murine model of myocardial infarction, Chung et al. [42•] studied cell viability and teratoma formation after embryonic stem cell (ESC) transplantation. They made use of a reporter gene designed to express antigens on the surface of embryonic stem cells (ESCs). By employing SPIOconjugated targeted monoclonal antibody (SPIO-MAb) against these reporter antigens, the viability, proliferation, and teratoma formation of transplanted ESCs could be quantified in vivo with MRI. The advantage of this reporter gene technique over direct stem cell labeling, is that the signal is enhanced when the stem cell successfully divides, thus, allowing quantification of subsequent stem cell proliferation.

\section{Endothelium}

Although the majority of preclinical endothelial imaging using iron oxide has examined large vessel vascular endothelium, which is beyond the scope of this review, there has been some recent work in the heart. Dall'Armellina et al. [43] employed a targeted system in characterizing the role of vascular cell adhesion molecule-1 (VCAM) that is known to play a crucial role in promoting endothelial inflammation after ischemia-reperfusion injury [44]. They described T2*- weighted MRI signal drop out with antibody-conjugated MPIO targeting VCAM-1 in mouse hearts following coronary artery ligation.

Grieve et al. [37] followed this work by adopting a similar protocol conjugating MPIO with monoclonal VCAM antibodies in a rodent model of MI. Extensive foci of signal voids were observed within the infarcted area on ex vivo $\mathrm{T} 2 *$ weighted MRI corresponding to MPIO deposition on histology. Supporting previous evidence of pan-myocardial inflammation after myocardial infarction, signal void was also detected in remote myocardium and pericardium.

\section{Conclusion and Future Applications}

Important and recent research has demonstrated that superparamagnetic particles of iron oxide can be used to detect cardiac inflammation in preclinical models of myocardial infarction, cardiac transplant rejection, myocarditis, as well as targeted imaging in stem cell therapy and apoptosis. These agents are able to provide a platform for both imaging and modulation of the disease process. Molecular MRI using iron nanoparticles, therefore, has the potential to develop into a powerful tool in diagnosing and treating inflammatory cardiac conditions in clinical medicine.

Recent translation of preclinical studies has already led to clinical applications examining myocardial inflammation following myocardial infarction. There have been concerns over the safety profile of iron oxide particles in the past, and at present there is only 1 iron oxide particle preparation available for clinical use: ferumoxytol (Rienso), a USPIO measuring 17-30 $\mathrm{nm}$. Interestingly Rienso is indicated for the intravenous treatment of iron deficiency anemia in adult patients with chronic kidney disease (CKD), and not its imaging properties.

Previous clinical preparations of superparamagnetic particles of iron oxide have not had a particularly successful track record. Another USPIO, ferumoxtran-10 (Sinerem), was previously approved in some European countries, but the manufacturer discontinued development due to concerns over unacceptably high false positive rate of detecting lymph node metastasis in patients with prostate cancer [45]. Other iron oxide nanoparticles including NC100500 (Clariscan), ferumoxides (Feridex in USA, Endorem in Europe), and ferucarbotran (Resovist) have all been discontinued over recent years. The latter 2 agents were the only 2 clinically approved SPIO agents for MRI, and it remains to be seen whether commercial companies will consider it economically viable to develop further iron oxide based agents for human imaging.

The natural progression following limited clinical studies using ferumoxytol, would be to characterize kinetics and dynamics of inflammatory cell recruitment to the myocardium not only following MI but also in other inflammatory 
myocardial processes including cardiac transplant rejection, myocarditis, and active cardiac sarcoidosis. If iron nanoparticle-enhanced MRI was able to diagnose and characterize inflammation in these diseases, it may eventually replace the need for cardiac biopsy. In conjugating iron oxide to various peptide, antibody, or gene therapy platforms, a new method of targeted therapy is a further potential opportunity for the clinic. This novel imaging tool may therefore prove a useful platform not only for diagnostic and disease monitoring purposes, but may even provide a treatment approach in these conditions.

Funding The British Heart Foundation (BHF) and Chief Scientist Office (CSO) have provided grants supporting work in this field at our center carried out by CS, JR and DEN. Work currently carried out by CS and DEN is supported by grants from the the BHF (FS/12/83/29781) and the CSO (ETM/266). JR was supported by a BHF Clinical PhD Training Fellowship (FS/07/060). DEN is supported by the British Heart Foundation $(\mathrm{CH} / 09 / 002)$

\section{Compliance with Ethics Guidelines}

Conflict of Interest Colin G Stirrat, David E Newby, Jennifer MJ Robson, and Maurits A Jansen declare that they have no conflict of interest.

Human and Animal Rights and Informed Consent This article does not contain any studies with human or animal subjects performed by any of the authors.

\section{References}

Papers of particular interest, published recently, have been highlighted as:

- Of importance

-• Of major importance

1. Cooper LT, Baughman KL, Feldman AM, Frustaci A, Jessup M, Kuhl $\mathrm{U}$, et al. The role of endomyocardial biopsy in the management of cardiovascular disease. A scientific statement from the American Heart Association, the American College of Cardiology, and the European Society of Cardiology Endorsed by the Heart Failure Society of America and the Heart Failure Association of the European Society of Cardiology. J Am Coll Cardiol. 2007;50:1914-31.

2. Wang Y-XJ, Hussain SM, Krestin GP. Superparamagnetic iron oxide contrast agents: physicochemical characteristics and applications in MR imaging. Eur Radiol. 2001;11:2319-31.

3. Wang Y-XJ. Superparamagnetic iron oxide based MRI contrast agents: current status of clinical application. Quant Imaging Med Surg. 2011;1:35-40.

4. Allkemper T, Bremer C, Matuszewski L, Ebert W, Reimer P. Contrast-enhanced blood-pool MR angiography with optimized iron oxides: effect of size and dose on vascular contrast enhancement in rabbits. Radiology. 2002;223:432-8.

5. Mandeville JB, Moore J, Chesler DA, Garrido L, Weissleder R, Weisskoff RM. Dynamic liver imaging with iron oxide agents: effects of size and biodistribution on contrast. Magn Reson Med. 1997;37:885-90
6. Hahn PF, Stark DD, Lewis JM, Saini S, Elizondo G, Weissleder R, et al. First clinical trial of a new superparamagnetic iron oxide for use as an oral gastrointestinal contrast agent in MR imaging. Radiology. 1990;175:695-700.

7. Saini S, Stark DD, Wittenberg J, Brady TJ, Ferrucci JT. Ferrite particles: a superparamagnetic MR contrast agent for the reticuloendothelial system. Radiology. 1987;162:211-6.

8. Rogers JM, Lewis J, Josephson L. Visualization of superior mesenteric lymph nodes by the combined oral and intravenous administration of the ultrasmall superparamagnetic iron oxide, AMI-227. Magn Reson Imaging. 1994;12:1161-5.

9. Zimmer C, Weissleder R, O'Connor D, LaPointe L, Brady TJ, Enochs WS. Cerebral iron oxide distribution: in vivo mapping with MR imaging. Radiology. 1995;196:521-7.

10. Canet E, Revel D, Forrat R, Baldy-Porcher C, de Lorgeril M, Sebbag L, et al. Superparamagnetic iron oxide particles and positive enhancement for myocardial perfusion studies assessed by subsecond T1-weighted MRI. Magn Reson Imaging. 1993;11: $1139-45$.

11. Trillaud H, Degrèze $P$, Mesplède $Y$, Douws $C$, Palussière J, Grenier N. Evaluation of experimentally induced renal hypoperfusion using iron oxide particles and fast magnetic resonance imaging. Acad Radiol. 1995;2:293-9.

12. McAteer MA, Akhtar AM, von Zur Muhlen C, Choudhury RP. An approach to molecular imaging of atherosclerosis, thrombosis, and vascular inflammation using microparticles of iron oxide. Atherosclerosis. 2010;209:18-27.

13. Wagner S, Schnorr J, Pilgrimm H, Hamm B, Taupitz M. Monomercoated very small superparamagnetic iron oxide particles as contrast medium for magnetic resonance imaging: preclinical in vivo characterization. Invest Radiol. 2002;37:167-77.

14. Taylor AM, Panting JR, Keegan J, Gatehouse PD, Amin D, Jhooti $\mathrm{P}$, et al. Safety and preliminary findings with the intravascular contrast agent NC100150 injection for MR coronary angiography. J Magn Reson Imaging. 1999;9:220-7.

15. Lind K, Kresse M, Debus NP, Müller RH. A novel formulation for superparamagnetic iron oxide (SPIO) particles enhancing MR lymphography: comparison of physicochemical properties and the in vivo behaviour. J Drug Target. 2002;10:221-30.

16. Clément $\mathrm{O}$, Siauve N, Cuénod CA, Frija G. Liver imaging with ferumoxides (Feridex): fundamentals, controversies, and practical aspects. Top Magn Reson Imaging. 1998;9:167-82.

17. Bourrinet $\mathrm{P}$, Bengele HH, Bonnemain B, Dencausse A, Idee J-M, Jacobs PM, et al. Preclinical safety and pharmacokinetic profile of ferumoxtran-10, an ultrasmall superparamagnetic iron oxide magnetic resonance contrast agent. Invest Radiol. 2006;41:313-24.

18. Yang Y, Yang Y, Yanasak N, Schumacher A, Hu TCC. Temporal and noninvasive monitoring of inflammatory - cell infiltration to myocardial infarction sites using micrometer - sized iron oxide particles. Magn Reson Med. 2010;63:33-40.

19. Ye Q, Wu YL, Foley LM, Hitchens TK, Eytan DF, Shirwan H, et al. Longitudinal tracking of recipient macrophages in a rat chronic cardiac allograft rejection model with noninvasive magnetic resonance imaging using micrometer-sized paramagnetic iron oxide particles. Circulation. 2008;118:149-56.

20. Briley-Saebo KC, Johansson LO, Hustvedt SO, Haldorsen AG, Bjørnerud A, Fayad ZA, et al. Clearance of iron oxide particles in rat liver: effect of hydrated particle size and coating material on liver metabolism. Invest Radiol. 2006;41:560-71.

21. Shapiro EM, Sharer K, Skrtic S, Koretsky AP. In vivo detection of single cells by MRI. Magn Reson Med. 2006;55:242-9.

22. Shapiro EM, Skrtic S, Sharer K, Hill JM, Dunbar CE, Koretsky AP. MRI detection of single particles for cellular imaging. Proc Natl Acad Sci U S A. 2004;101:10901-6.

23. Hertz MI, Aurora P, Christie JD, Dobbels F, Edwards LB, Kirk R, et al. Scientific Registry of the International Society for Heart and 
Lung Transplantation: introduction to the 2009 Annual Reports. J Heart Lung Transplant. 2009;28:989-92.

24. Stewart S, Winters GL, Fishbein MC, Tazelaar HD, Kobashigawa J, Abrams J, et al. Revision of the 1990 working formulation for the standardization of nomenclature in the diagnosis of heart rejection. J Heart Lung Transplant. 2005;24:1710-20.

25. Kanno S, Wu Y-JL, Lee PC, Dodd SJ, Williams M, Griffith BP, et al. Macrophage accumulation associated with rat cardiac allograft rejection detected by magnetic resonance imaging with ultrasmall superparamagnetic iron oxide particles. Circulation. 2001;104:934-8

26. Wu YL, Ye Q, Sato K, Foley LM, Hitchens TK, Ho C. Noninvasive evaluation of cardiac allograft rejection by cellular and functional cardiac magnetic resonance. JACC Cardiovasc Imaging. 2009;2: 731-41.

27. Guo Y, Chen W, Wang W, Shen J, Guo R, Gong F, et al. Simultaneous diagnosis and gene therapy of immuno-rejection in rat allogeneic heart transplantation model using a t-cell-targeted theranostic nanosystem. ACS Nano. 2012;121204131551005. Novel "theranostic" nanosystem allowing diagnosis and treatment (via gene therapy) of allogeneic cardiac transplantation rejection in rats.

28. Narula J, Acio ER, Narula N, Samuels LE, Fyfe B, Wood D, et al. Annexin-V imaging for noninvasive detection of cardiac allograft rejection. Nat Med. 2001;7:1347-52. doi:10.1038/nm1101-1241 [Pub online: Nov 1, 2001].

29. Dash R, Chung J, Chan T, Yamada M, Barral J, Nishimura D, et al. A molecular MRI probe to detect treatment of cardiac apoptosis in vivo. Magn Reson Med. 2011;66:1152-62.

30. Wunderbaldinger P, Josephson L, Weissleder R. Crosslinked iron oxides (CLIO): a new platform for the development of targeted MR contrast agents. Acad Radiol. 2002;9 Suppl 2:S304-6.

31. Schellenberger EA, Högemann D, Josephson L, Weissleder R. Annexin V-CLIO: a nanoparticle for detecting apoptosis by MRI. Acad Radiol. 2002;9 Suppl 2:S310-1.

32. Sosnovik DE, Schellenberger EA, Nahrendorf M, Novikov MS, Matsui T, Dai G, et al. Magnetic resonance imaging of cardiomyocyte apoptosis with a novel magneto- optical nanoparticle. Magn Reson Med. 2005;54:718-24.

33. Sosnovik DE, Garanger E, Aikawa E, Nahrendorf M, Figuiredo JL, Dai G, et al. Molecular MRI of cardiomyocyte apoptosis with simultaneous delayed-enhancement MRI distinguishes apoptotic and necrotic myocytes in vivo potential for midmyocardial salvage in acute ischemia. Circ Cardiovasc Imaging. 2009;2:460-7.

34. Yang Y, Liu J, Yang Y, Cho SH, Hu TCC. Assessment of cell infiltration in myocardial infarction: a dose-dependent study using micrometer-sized iron oxide particles. Magn Reson Med. 2011;66: 1353-61.

35.• Alam SR, Shah ASV, Richards J, Lang NN, Barnes G, Joshi N, et al. Ultrasmall superparamagnetic particles of iron oxide in patients with acute myocardial infarction: early clinical experience. Circ Cardiovasc Imaging. 2012;5:559-65. First human studies evaluating uptake of iron nanoparticles after myocardial infarction.

36.• Yilmaz A, Dengler MA, van der Kuip H, Yildiz H, Rosch S, Klumpp S, et al. Imaging of myocardial infarction using ultrasmall superparamagnetic iron oxide nanoparticles: a human study using a multi-parametric cardiovascular magnetic resonance imaging approach. Eur Heart J. 2013;34:462-75. First human studies evaluating uptake of iron nanoparticles after myocardial infarction.

37. Grieve SM, Lønborg J, Mazhar J, Tan TC, Ho E, Liu C-C, et al. Cardiac magnetic resonance imaging of rapid VCAM-1 upregulation in myocardial ischemia-reperfusion injury. Eur Biophys J. 2012;42:61-70.

38. Ruparelia N, Digby JE, Jefferson A, Medway DJ, Neubauer S, Lygate CA, et al. Myocardial infarction causes inflammation and leukocyte recruitment at remote sites in the myocardium and in the renal glomerulus. Inflamm Res. 2013;62:515-25.

39. Moon H, Park HE, Kang J, Lee H, Cheong C, Lim YT, et al. Noninvasive assessment of myocardial inflammation by cardiovascular magnetic resonance in a rat model of experimental autoimmune myocarditis. Circulation. 2012;125:2603-12.

40. Kraitchman DL. In vivo magnetic resonance imaging of mesenchymal stem cells in myocardial infarction. Circulation. 2003;107: 2290-3.

41. Lu M, Zhao S, Liu Q, Jiang S, Song P, Qian H, et al. Transplantation with autologous mesenchymal stem cells after acute myocardial infarction evaluated by magnetic resonance imaging: an experimental study. J Thorac Imaging. 2012;27:125-35.

42. Chung J, Kee K, Barral JK, Dash R, Kosuge H, Wang X, et al. In vivo molecular MRI of cell survival and teratoma formation following embryonic stem cell transplantation into the injured murine myocardium. Magn Reson Med. 2011;66:1374-81. Imaging of proliferation and teratoma formation after embryonic stem cell transplantation. A reporter gene expressing antigen on the surface of stem cells allowed quantification of viability and proliferation of stem cells whilst monitoring for teratoma formation.

43. Dall'Armellina E, Lygate CA, Mcateer M. Ex vivo and in vivo MR imaging of ischemia reperfusion injury in mouse hearts using microparticles of iron oxide targeting VCAM-1. Proceedings of the 18th Annual Meeting ISMRM, Stockholm, Sweden, 2010;3637.

44. Gabrielsen A, Lawler PR, Yongzhong W, Steinbrüchel D, Blagoja $\mathrm{D}$, Paulsson-Berne $\mathrm{G}$, et al. Gene expression signals involved in ischemic injury, extracellular matrix composition and fibrosis defined by global mRNA profiling of the human left ventricular myocardium. J Mol Cell Cardiol. 2007;42:870-83.

45. Heesakkers RAM, Jager GJ, Hövels AM, de Hoop B, van den Bosch HCM, Raat F, et al. Prostate cancer: detection of lymph node metastases outside the routine surgical area with ferumoxtran-10enhanced MR imaging. Radiology. 2009;251:408-14. 


\section{Ultra-small superparamagnetic particles of iron oxide in magnetic resonance imaging of cardiovascular disease}

\author{
Colin G Stirrat ${ }^{1}$ \\ Alex T Vesey' \\ Olivia MB McBride' \\ Jennifer MJ Robson' \\ Shirjel R Alam' \\ William A Wallace ${ }^{2}$ \\ Scott I Semple ${ }^{1,3}$ \\ Peter A Henriksen' \\ David E Newby'
}

'British Heart Foundation Centre for Cardiovascular Science, University of Edinburgh, Edinburgh, UK; ${ }^{2}$ Department of Pathology, University of Edinburgh, Edinburgh, UK; ${ }^{3} \mathrm{Clinical}$ Research Imaging Centre, University of Edinburgh, Edinburgh, UK
Correspondence: Colin G Stirrat British Heart Foundation Centre for Cardiovascular Science, Room SU 305, Chancellor's Building, University of Edinburgh, 49 Little France Crescent, Edinburgh EHI6 4SB, UK

Tel +44 I3I 2426515

$\mathrm{Fax}+44$ I3| 2426379

Email colin.stirrat@ed.ac.uk

\begin{abstract}
Ultra-small superparamagnetic particles of iron oxide (USPIO) are iron-oxide based contrast agents that enhance and complement in vivo magnetic resonance imaging (MRI) by shortening $\mathrm{T} 1, \mathrm{~T} 2$, and $\mathrm{T} 2 *$ relaxation times. USPIO can be employed to provide immediate blood pool contrast, or to act as subsequent markers of cellular inflammation through uptake by inflammatory cells. They can also be targeted to specific cell-surface markers using antibody or ligand labeling. This review will discuss the application of USPIO contrast in MRI studies of cardiovascular disease.
\end{abstract}

Keywords: cardiac, aortic, MRI, USPIO, carotid, vascular, molecular imaging

\section{Introduction}

Beyond the anatomical and morphological information afforded by standard clinical cardiovascular imaging techniques, it is increasingly recognized that additional information regarding the underlying biological processes involved would be valuable in a range of cardiovascular diseases to aid understanding of disease pathogenesis, provide diagnostic information, predict disease progression, and to monitor response to treatment. Novel molecular and cellular imaging techniques can identify metabolic and biological processes that are critical to disease pathogenesis, and therefore have the potential to predict clinical events and guide therapeutic intervention.

A range of potential imaging modalities exists (Table 1). Magnetic resonance imaging (MRI) has a number of advantages including excellent discrimination of soft tissue components, good spatial resolution and avoidance of ionizing radiation, a particular consideration if serial imaging is envisaged.

Iron oxide particles are a group of MRI contrast agents that can be used for vascular, molecular and cellular imaging. ${ }^{1}$ Iron oxide particles can be categorized according to their size: nano-sized very small superparamagnetic particles of iron oxide (VSPIO, $<20 \mathrm{~nm}$ in diameter), ultra-small superparamagnetic particles of iron oxide (USPIO, 20-50 nm in diameter), small superparamagnetic particles of iron oxide (SPIO, $60-250 \mathrm{~nm}$ in diameter), and micro-sized particles of iron oxide (MPIO, 1-8 $\mu \mathrm{m}$ in diameter). VSPIO and USPIO are often classified together (as USPIO) due to similar size. Superparamagnetism occurs when the size of the iron oxide crystal is smaller than that of ferromagnetic domains (approximately $30 \mathrm{~nm}$ ). Under the influence of a strong external magnetic field, such as within an MRI scanner, iron oxide nanoparticles have a large magnetic moment, but unlike larger ferromagnetic particles, this magnetic moment is lost when the field is removed. 
Table I Summary of commonly used molecular imaging modalities

\begin{tabular}{|c|c|c|c|c|c|c|}
\hline Modality & Spatial resolution & Temporal resolution & Sensitivity & Penetration & Radiation & Cost \\
\hline MRI & $10-100$ micron & High & Low & Good & No & High \\
\hline US & 50 micron & High & Medium & $\mathrm{cm}$ & No & Medium \\
\hline SPECT & $\mathrm{I}-2 \mathrm{~mm}$ & Low & Medium & Good & Yes & Medium \\
\hline PET & $\mathrm{I}-2 \mathrm{~mm}$ & Low & High & Good & Yes & High \\
\hline Fluorescence & $2-3 \mathrm{~mm}$ & High & High & $<\mathrm{l} \mathrm{cm}$ & Yes/no & Low \\
\hline
\end{tabular}

Abbreviations: MRI, magnetic resonance imaging; US, ultrasound; SPECT, single-photon emission computerised tomography; PET, positron emission tomography.

USPIO generally consist of an iron oxide core surrounded by a coating, usually of carbohydrate or polymer construction, with a total particle diameter of less than $50 \mathrm{~nm}$. The purpose of the coating is to prevent aggregation, to retain particles within a colloidal suspension and to provide a surface for conjugation of drug molecules and targeting ligands. A range of particles exists varying in size and coating; these two properties are the key determinants of biodistribution, magnetic behavior, and thus the potential applications of the particle. ${ }^{2,3}$ The majority of proposed applications for iron oxide nanoparticles utilize particles within the ultra-small and small size ranges.

Following administration, cells of the reticuloendothelial system including inflammatory macrophages internalize iron oxide nanoparticles. Neutrophils and structural endothelial and tumor cells also take up iron nanoparticles. Larger SPIO particles are identified and rapidly engulfed, principally by resident macrophages in the lymphoreticular organs including the liver, spleen, and lymph nodes. In contrast, USPIO particles are much smaller and therefore less readily recognized by cells, escaping immediate phagocytosis and resulting in a much longer half-life within the blood pool compared to larger particles (up to 36 hours for $\mathrm{USPIO}^{4}$ versus as low as 2 hours for larger $\mathrm{SPIO}^{5}$ in humans). The biodistribution of USPIO is individual to each particle but a blood pool half-life of several hours is expected with distribution predominantly in the spleen, liver, bone marrow, and lymph nodes, before being cleared within a matter of a few days. Cytotoxicity data for iron nanoparticles is limited but is not found until exposure to high levels of iron and is also dependent on factors such as surface coating or its breakdown products, composition of the cell medium, and protein-iron nanoparticle interaction. ${ }^{6-9}$

Whilst circulating within the blood pool, USPIO are small enough to migrate passively across the endothelium ${ }^{1}$ especially in regions of the vasculature where there is loss of endothelial integrity. Once present within tissues, nanoparticles are concentrated and engulfed by resident macrophages through phagocytosis and pinocytosis resulting in their concentration within inflamed tissues. This concentration results in magnetic cooperativity, enhancing their superparamagnetic effects, markedly shortening the $\mathrm{T} 2 *$ relaxation time and creating local signal deficits on $\mathrm{T} 2$ - and $\mathrm{T} 2 *$-weighted images. Thus USPIO can highlight areas of tissue inflammation on MRI. ${ }^{10}$

Earlier studies using iron nanoparticles employed semiquantitative methods to calculate reduction in signal intensity consistent with iron accumulation, however this is liable to variations in signal across the image relative to proximity to surface coils between visits, and also assumes that signal intensity in the control tissue is consistent between both pre- and post-USPIO scans. Calculation of changes in $\mathrm{T} 2$ and $\mathrm{T} 2 *$ relaxivity values has been adopted for a more sensitive and objective approach. The T2 and T2* relaxivity values, are the initial decay constants for the exponential decay of signal intensity with increasing echo time. Calculation and comparison of decay constants for each region of interest allows for a more objective and quantitative analysis approach and thus accounts for problems caused by variations in relaxivity values between regions and also MRI coil sensitivity between subject visits. In the presence of USPIO the signal decays more rapidly and the $\mathrm{T} 2$ and $\mathrm{T} 2 *$ relaxivity values are reduced. On the resulting images, particularly on $\mathrm{T} 2 *$-weighted images, a profound reduction in signal intensity is evident as a region of signal void (hypointense region) where USPIOs have accumulated (Figure 1).

Precise blood suppression during USPIO-enhanced MRI is important to reduce artifact into nearby myocardium,
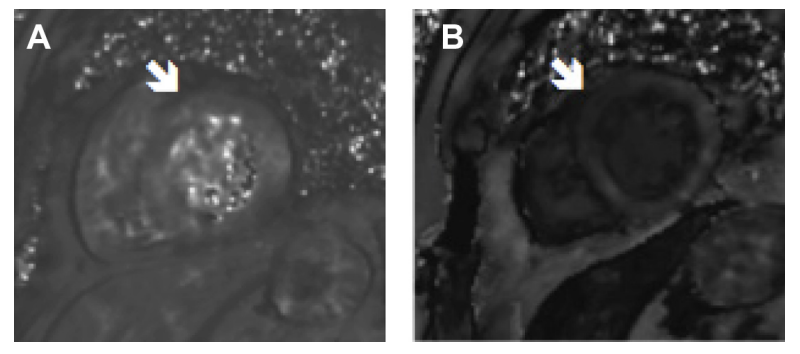

Figure I Patient imaged 3 days after an anterior myocardial infarct with T2*weighted magnetic resonance imaging.

Notes: Images show T2* maps of baseline pre-iron scan (A), and 24 hours following ferumoxytol (USPIO) administration (B). Iron uptake is seen as signal void (dark) in the region of the anterior infarct (arrow).

Abbreviation: USPIO, ultra-small superparamagnetic particles of iron oxide. 
which is often the region of interest under examination. Magnetization transfer between the blood pool and myocardium, and also flow-related blood pool artifact, may compromise the quality of T2* data. Double inversion techniques are commonly used to suppress blood signal in most cardiac MRI examinations. However, double inversion techniques do not account for changes in blood pool USPIO related T1 shortening. Therefore, more complicated inversion strategies (such as a quadrature inversion technique) may be required to fully null blood signal in both pre- and post-USPIO data, although these inversion techniques are less commonly available on clinical systems and so are not widely used.

Here, we focus on the use of USPIO in cardiovascular imaging, discussing their application as a blood pool contrast agent, a tracer highlighting inflammatory cells of the reticuloendothelial system, and in targeted imaging conjugated to a specific antibody or peptide (Table 2).

\section{Cardiac imaging Blood pool contrast}

USPIO have been used successfully as coronary and ventricular MRI blood pool contrast agents for over a decade as they have longer blood pool circulating time compared to standard MRI angiographic agents such as gadolinium. In 1997, Stillman et $\mathrm{l}^{11}$ demonstrated improved myocardial to blood pool border definition with ferumoxtran-10 on T1-weighted MRI cine images. Image intensity gradients at the myocardial to blood pool interface increased significantly in both long and short axis views. At the time it was thought that ferumoxtran-10 would improve automated ventricular cavity edge detection.

Table 2 Summary of USPIO studies discussed in this article

\begin{tabular}{|c|c|c|c|c|c|c|c|}
\hline Agent name & $\begin{array}{l}\text { Iron oxide cored } \\
\text { diameter, nm } \\
\text { (total diameter) }\end{array}$ & Coating & $\begin{array}{l}\text { Magnet } \\
\text { strength }\end{array}$ & $\begin{array}{l}\text { Administration } \\
\text { site }\end{array}$ & $\begin{array}{l}\text { Clinical/ } \\
\text { preclinical }\end{array}$ & Application & Reference \\
\hline Ferumoxtran-10 & $6.3(35)$ & Dextran & $1.5 \mathrm{~T}$ & Intravenous & Clinical & Ventricular contrast & 11 \\
\hline (AMI-227, & & & $1.5 \mathrm{~T}$ & Intravenous & Preclinical & Cardiac transplant & 26,28 \\
\hline Combidex ${ }^{\circledR}$, & & & & & & rejection & \\
\hline \multirow[t]{8}{*}{ Sinerem ${ }^{\circledR}$ ) } & & & $7 \mathrm{~T}$ & Intramyocardial & Preclinical & Stem cells & 36 \\
\hline & & & $1.5 \mathrm{~T}$ & Intravenous & Preclinical & Atherosclerosis & 50,51 \\
\hline & & & $1.5 \mathrm{~T}$ & Intravenous & Clinical & Atherosclerosis & $\begin{array}{l}52-55 \\
57-59,62\end{array}$ \\
\hline & & & $\mathrm{I} .5 \mathrm{~T}$ & Intravenous & Clinical & Atherosclerosis + AAA & 76 \\
\hline & & & $1.5 \mathrm{~T}$ & Intravenous & Clinical & Aortic + iliac aneurysms & 77 \\
\hline & & & $1.5 \mathrm{~T}$ & Intravenous & Clinical & AAA & 78 \\
\hline & & & $3 \mathrm{~T}$ & Intravenous & Clinical & AAA & 79 \\
\hline & & & I.5 T & Intravenous & Clinical & Lymph node metastases & 80 \\
\hline $\mathrm{NCIO0I50}$ & $6.43(20)$ & Dextran & $0.5 / 1.5 \mathrm{~T}$ & Intravenous & Clinical & Ventricular contrast & $12-15$ \\
\hline \multirow[t]{5}{*}{$\left(\right.$ Clariscan $\left.^{\mathrm{TM}}\right)$} & & & $\mathrm{I} .5 \mathrm{~T}$ & Intravenous & Preclinical & Coronary contrast & 16 \\
\hline & & & $1.5 \mathrm{~T}$ & Intravenous & Clinical & Coronary contrast & 17,18 \\
\hline & & & $1.5 \mathrm{~T}$ & Intravenous & Preclinical & Myocardial contrast & 19,20 \\
\hline & & & $2 \mathrm{~T}$ & Intravenous & Preclinical & Myocardial contrast & 21 \\
\hline & & & $1.5 \mathrm{~T}$ & Intravenous & Preclinical & $\begin{array}{l}\text { Cardiac transplant } \\
\text { rejection }\end{array}$ & 25,26 \\
\hline$n / a$ & n/a (27) & Dextran & $4.7 \mathrm{~T}$ & Intravenous & Preclinical & $\begin{array}{l}\text { Cardiac transplant } \\
\text { rejection }\end{array}$ & 24,27 \\
\hline$n / a$ & $6(n / a)$ & Polymer & $\mathrm{I} .5 \mathrm{~T}$ & Intravenous & Preclinical & $\begin{array}{l}\text { Cardiac allograft } \\
\text { rejection }\end{array}$ & 30 \\
\hline \multirow[t]{5}{*}{ n/a (CLIO) } & $3-5(15-20)$ & Dextran & $9.4 \mathrm{~T}$ & Intravenous & Preclinical & Myocardial infarction & 32 \\
\hline & & & $9.4 \mathrm{~T}$ & Intramyocardial & Preclinical & Stem cells & 37 \\
\hline & & & $\mathrm{I} .5 \mathrm{~T}$ & $\begin{array}{l}\text { Gel Phantom/ } \\
\text { intramyocardial }\end{array}$ & $\begin{array}{l}\text { In vitro/ } \\
\text { ex vivo }\end{array}$ & Stem cells & 39 \\
\hline & & & $\mathrm{n} / \mathrm{a}$ & n/a (in-vitro) & In vitro & Apoptosis & 40 \\
\hline & & & $9.4 \mathrm{~T}$ & Intravenous & Preclinical & Apoptosis & 41,42 \\
\hline Ferumoxytol & $3.25(|7-3|)$ & Dextran & $3 \mathrm{~T}$ & Intravenous & Clinical & Myocardial infarction & 33 \\
\hline (Rienso ${ }^{\circledR}$, & & & $1.5 \mathrm{~T}$ & Intravenous & Clinical & Myocardial infarction & 34 \\
\hline \multicolumn{8}{|l|}{ Feraheme $^{\circledR}$ ) } \\
\hline $\mathrm{n} / \mathrm{a}$ & n/a (7.5) & Amine silane & $3 \mathrm{~T}$ & Intracoronary & Preclinical & Stem cells & 38 \\
\hline $\mathrm{n} / \mathrm{a}$ & $2-7(15-40)$ & Polysaccharide & $1.5 \mathrm{~T}$ & Intravenous & Preclinical & Atherosclerosis & 49 \\
\hline
\end{tabular}

Note: Rienso ${ }^{\circledR}$; Takeda Italia S.p.A, Rome, Italy.

Abbreviations: USPIO, ultra-small superparamagnetic particles of iron oxide; AAA, abdominal aortic aneurysms; CLIO, cross-linked particles of iron oxide; n/a, not available. 
This was soon followed up by Taylor et $\mathrm{al}^{12}$ who used an alternative USPIO agent, NC100150 (Clariscan ${ }^{\mathrm{TM}}$ ) with a blood pool half-life of over 2 hours, to aid ventricular cavity edge detection using MRI. They administered NC100150 to 18 healthy male volunteers and examined optimized cine imaging sequences. At higher doses ( 3 and $4 \mathrm{mg} \mathrm{Fe} / \mathrm{kg}$ ) a reduction in signal intensity was seen in the blood pool enabling enhanced detection of the left ventricle blood pool to myocardial interface thus improving functional assessment of the left ventricle. The finding that NC100150 improves myocardial blood pool definition has since been repeated in other human studies. ${ }^{13-15}$

NC100150 has been used extensively as a coronary contrast agent for MRI coronary angiography (MRCA) with mixed results. Johansson et al compared invasive X-ray angiography with T1-weighted MRCA using NC100150 in pigs with coronary artery stenosis. ${ }^{16}$ Although the sample size was small $(n=6)$, the location and severity grading of stenoses on MRCA correlated well with X-ray angiography findings in all animals. Within a few years, Sandstede et a ${ }^{17}$ carried out a feasibility study using the same contrast agent in patients with coronary artery disease. Proximal segments of coronary arteries could be visualized but distal visualization was poor. Furthermore, no improvement in image quality was seen when an increased USPIO dose was used and only six of eight proximal coronary stenoses seen on conventional angiography were detected on the NC100150 contrast enhanced scan.

Klein et $\mathrm{al}^{18}$ conducted an experiment comparing NC100150 enhanced MRI with unenhanced MRI imaging, again yielding mixed results. Although the visible length of mid and distal portions of the coronary artery improved on USPIO-enhanced imaging, there was no improvement when assessing the proximal portion of the artery. Furthermore, only eight of the eleven significant coronary stenoses $(>50 \%)$ were detected on USPIO-enhanced MRI compared to six on the unenhanced scan.

Soon after the development of USPIO as a blood pool contrast agent for ventricular cavity and coronary imaging, attempts were made to illustrate myocardial viability with conflicting results. Kroft et al ${ }^{19}$ administered ferumoxtran-10 (AMI-227, Sinerem ${ }^{\circledR}$ ) in a pig model of myocardial infarction (MI) and then imaged using T1-weighted MRI with a $1.5 \mathrm{~T}$ scanner. Interim imaging failed to detect USPIO in the viable myocardium, likely due to low dose, fast elimination time of the contrast agent, and a low field strength scanner. Increasing the magnetic field strength provides improved signal-to-noise ratio, greater spatial resolution, and higher sensitivity for detecting lower concentrations of iron, which is advantageous in the detection of USPIO.

Bjerner et $\mathrm{a}^{20}$ had more success using NC100150 in a similar model. Using T1-, T2-, and T2*-weighted imaging, they found that T1-weighted MRI with doses of 4 and $8 \mathrm{mg}$ $\mathrm{Fe} / \mathrm{kg}$ produced the best evaluation of perfused myocardium after histological validation against areas of fluorescein stain uptake on ex vivo histological analysis.

The use of USPIO as a myocardial tissue contrast agent was extended to a model of ischemia-reperfusion in rats to evaluate microvascular injury in reperfused myocardium. Krombach et $\mathrm{al}^{21}$ employed coronary ligation for 45 minutes and 3 hours of reperfusion, and administered NC100150, to help define regions of microvascular injury and obstruction after MI. USPIO distributes by diffusion in the extravascular space of injured myocardium with damaged, leaky vasculature. They found that USPIO-enhanced imaging provided some indication of transmural and non-transmural microvascular injury. This imaging approach tended to overestimate the area of true infarction when compared to triphenyltetrazolium chloride stain, and underestimate the area of myocardium deemed "at risk" of infarction determined by redo coronary ligation and intravenous phthalocyanine blue dye injection.

\section{Inflammation}

Endomyocardial biopsy is the "gold standard" diagnostic investigation for inflammatory diseases of the myocardium. This does carry the potential for life-threatening complication, ${ }^{22}$ and sampling error is common due to the focal nature and spatial heterogeneity of underlying inflammatory processes. This procedure is rarely performed outside specialist tertiary referral centers.

\section{Transplant rejection}

Cardiac transplantation is a life prolonging treatment option for end-stage cardiac disease. Transplant rejection is a major threat to the allograft, occurring in $20 \%-30 \%$ of recipients in the first year. ${ }^{23}$ Rejection can occur at any stage after transplantation and causes significant morbidity and mortality. Serial surveillance myocardial biopsies are routinely undertaken as rejection is notoriously difficult to diagnose using existing non-invasive imaging methods.

Kanno et $\mathrm{al}^{24}$ exploited the finding that iron nanoparticles accumulated within the cytoplasm of macrophages, and detected organ rejection using MRI and dextran-coated USPIO in rats with evidence of cardiac allograft rejection. 
Histopathology confirmed active rejection and identified USPIO uptake confined to macrophages that had accumulated within the rejecting cardiac allograft. Rats that received more prolonged immunosuppression had a decline in the rate of signal intensity reduction, suggesting less inflammation and indicating that treatment response could be monitored with this method.

Subsequent studies have confirmed the value of using USPIO to detect acute cardiac rejection in rats, ${ }^{25-27}$ although ferumoxtran-10 proved inferior to $\mathrm{NC100150}$ at detecting rejection ${ }^{26}$ and was unable to detect acute cardiac rejection in rats in another study. ${ }^{28}$ In the latter study, ferumoxtran-10 was given at two doses of $2 \mathrm{mg}$ and $11 \mathrm{mg} \mathrm{Fe} / \mathrm{kg}$ with T2*weighted MRI conducted using a $1.5 \mathrm{~T}$ scanner at postoperative day 7, 24 hours after USPIO administration. The failure to detect USPIO was thought to be due to the low field strength magnet of the MRI scanner.

Iron oxide particles can be conjugated to a specific antibody or peptide to target pathophysiological processes. ${ }^{29}$ Recent work by Guo et al ${ }^{30}$ targeted $\mathrm{T}$ cells that play a central role in acute cardiac allograft rejection. They built on work performed using ligand targeting of cells to employ a "theranostic" strategy, combining diagnostic imaging with administration of therapy within the one system (Figure 2). They targeted $\mathrm{T}$ cells in the endocardium of rejecting rat hearts using a CD-3 antibody-targeted multifunctional polymeric nanocarrier. This polyplex nanoparticle was further conjugated to a therapeutic gene ( $p D N A-D G K a l p h a$ ) enabling simultaneous imaging and immune modulation of CD-3 expressing T cells.

\section{MI}

The development of cross-linked particles of iron oxide $(\mathrm{CLIO})^{31}$ allowed targeted cardiac inflammatory imaging. Cross-linking and amination of the USPIO dextran chains encourages circumferential adherence of the dextran coat to the iron nanoparticle providing chemical stability and improved conjugation. ${ }^{31}$ Sosnovik et al ${ }^{32}$ conjugated CLIO with cyanine 5.5 dye creating a "magnetofluorescent" particle allowing co-registration of MRI and fluorescence molecular tomography data. They administered CLIO-Cy5.5 48 hours after left coronary artery ligation in a mouse model of MI with dual modality imaging 48 hours later. The accumulation of CLIO-Cy5.5 within infarcted regions of myocardium was clearly seen on fluorescence and MRI images that were not present on control mice (mice injected with CLIO-Cy5.5 but a "sham" operation). In vivo imaging findings were subsequently confirmed with histology. Examination of infarcted

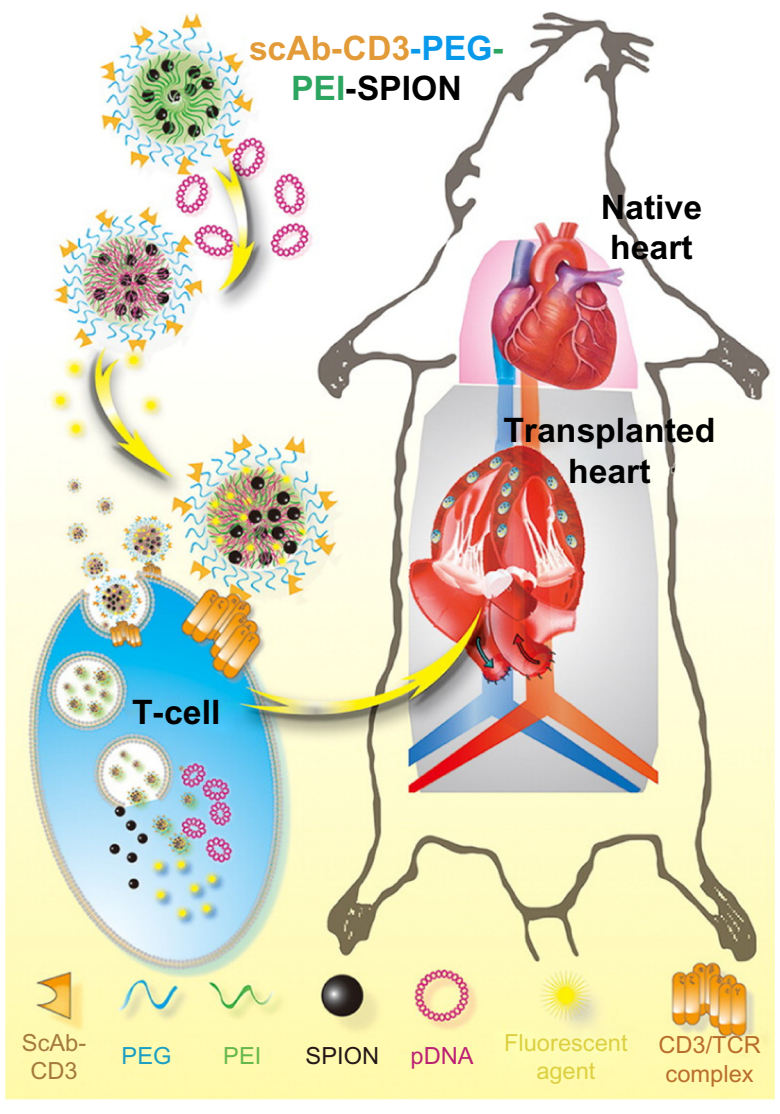

Figure 2 Schematic diagram of therapeutic process of magnetic targeting polyplex scAbCD3-PEG-g-PEI-SPION in vitro and in vivo.

Notes: Reprinted with permission from Guo Y, Chen W, Wang W, et al. Simultaneous diagnosis and gene therapy of immuno-rejection in rat allogeneic heart transplantation model using a T-cell-targeted theranostic nanosystem. ACS Nano. 20I2;6(I2): 10646-10657. Copyright (C) 2012 American Chemical Society. ${ }^{30}$

Abbreviations: ScAb-CD3, CD3 single chain antibody; PEG, polyethylene glycol; PEI, polyethylenimine; SPION, superparamagnetic iron oxide nanoparticles; pDNA, Plasmid DNA; TCR, T-cell receptor.

myocardium revealed a high degree of co-localization between inflammatory cell infiltrates on hematoxylin and eosin staining, increased signal intensity on fluorescence microscopy, and an abundance of macrophages on immunohistochemistry staining. This is consistent with uptake of CLIO-Cy5.5 by macrophages infiltrating the infarcted myocardium.

Clinical applications of USPIO in highlighting myocardial inflammation after MI have since emerged following the discovery that inflammation around the time of MI affects both the extent of myocardial salvage and recovery of left ventricular function. Our group ${ }^{33}$ used USPIO to image myocardial inflammation following acute MI. Within 48 hours following acute MI, patients underwent MRI scanning at baseline (pre-USPIO) before receiving an infusion of ferumoxytol (USPIO) with subsequent serial MRI scans thereafter. The infarct zone was defined by delayed enhancement with gadolinium. Following 
USPIO administration, there was a significant increase in $\mathrm{R} 2 *(\mathrm{R} 2 *=1 / \mathrm{T} 2 *)$ in the infarct zone, with smaller changes observed in the peri-infarct zone (surrounding areas of delayed enhancement) and even in remote myocardium when compared with control tissue (skeletal muscle). This suggested peri-infarct and remote myocardial inflammation, although no histological data were available to confirm this. Notably, the R2* value was greatest 24 hours following USPIO (ferumoxytol) infusion, and had declined by 48 hours suggesting that 24 hours post-infusion is a more sensitive imaging time than 48 hours post-infusion in this group of patients.

Yilmaz et $\mathrm{al}^{34}$ also detected a reduction in $\mathrm{T} 2 *$ value in the infarct as well as in the peri-infarct region, the remote myocardium, and the lymphoreticular system. On imaging the first three patients at 6, 24, 48 and 96 hours after ferumoxytol administration, they attempted to find the optimal time window to characterize inflammation within the myocardium. From these data, they suggest that signal attenuation was maximal at 24 hours but that the best combination of T2-weighted (hypoenhancement seen) and T2*-weighted images was obtained at 48 hours.

Histological data are required to address the outstanding question regarding distribution of USPIO within the myocardium and the relative contribution to changes in $\mathrm{T} 2 *$ of extracellular iron accumulating within the interstitium, capillary bed, and inflammatory macrophages.

The finding of $\mathrm{T} 2 *$ signal void in remote myocardium suggests a pan-myocardial inflammatory response in the context of a focal infarct. Grieve et $\mathrm{a}^{35}$ confirmed this finding in remote segments in a rat model of ischemia-reperfusion injury examining endovascular inflammation using a targeted larger iron oxide tracer (MPIO).

\section{Other cardiac imaging}

\section{Stem cells}

Current research endeavors are increasingly focusing on stem cell therapy following the discovery that pluripotent progenitor cells bear the capacity to differentiate into mature cardiac cells, in an attempt to restore function to organs with no regenerative capacity. There is a need to track stem cells following administration so that their distribution and fate can be determined. Cell labeling with iron nanoparticles is relatively simple, fast, inexpensive and is well described, although there have been few published studies using USPIO in cardiac stem cell imaging. Tracking of stem cells labeled with iron nanoparticles using MRI is still at the pre-clinical stage but offers high spatial resolution, excellent soft tissue detail, and avoids exposure to ionizing radiation.
Küstermann et $\mathrm{al}^{36}$ labeled cardiac progenitor cells expressing enhanced green fluorescent protein with ferumoxtran-10 and implanted the cells into murine myocardium injured through cryocoagulation or coronary artery ligation. Co-registration of USPIO and stem cells was confirmed by comparing high-resolution MRI images with Prussian Blue stain for iron on histology, and immunohistochemistry for enhanced green fluorescent protein. Difficulties were encountered using this approach as the signal attenuation of unlabeled ischemic myocardium was similar to that induced by USPIO-labeled cells in T2*-weighted images making detection of labeled cells within infarcted regions difficult. However, the authors subsequently found that the combination of proton density- and $\mathrm{T} 2 *$-weighted MRI sequences identified regions of myocardial injury and USPIO-labeled cells respectively - the larger area of signal attenuation in the $\mathrm{T} 2 *$-weighted images was caused by infiltration of the iron-labeled cells.

Chapon et $\mathrm{al}^{37}$ combined MRI and positron emission tomography to track transplanted stem cells after MI in rats providing anatomical, physiological, and metabolic information. They conjugated rat bone marrow-derived stem cells to CLIO and fluorescein dye enabling co-localization of stem cells to be assessed by in vivo MRI and ex vivo magnetic resonance spectroscopy. Stem cells were successfully detected on MRI and histology for the 6 week duration of the study, however positron emission tomography signs of metabolic activity (18F-fluorodeoxyglucose uptake) was only seen in the first week post-stem cell implantation. This may have been due to early, localized inflammation from stem cell injection or more likely death of the stem cells. A trend to an improvement in cardiac function was found after stem cell implantation, although this did not reach statistical significance.

Ma et $\mathrm{al}^{38}$ compared the ability of USPIO and MPIO in tracking stem cells via intracoronary injection, as opposed to a direct intramyocardial route, in a swine model of ischemiareperfusion injury. They found that similar labeling efficiency was found in both particles, and differentiation and proliferation of mesenchymal stem cells (MSCs) in vivo were not affected by labeling with either iron particle, confirmed by immunohistochemical assays showing similar protein expression. Hypointensity was seen in infarcted regions with both particles on $\mathrm{T} 2 *$-weighted MRI at 4 and 8 weeks and co-localized with regions of iron uptake seen on Prussian Blue staining. However there was no hypointensity detected in 2/7 of the MPIO labeled pigs and the authors suggested that USPIO labeling was more suited to intracoronary injection than the larger MPIO particle. 
Mathiasen et a ${ }^{39}$ recently sought to determine the MRI detection limits of CLIO labeled human MSCs using gelphantoms in a clinical $1.5 \mathrm{~T}$ MR scanner. They determined the detection limit to be 250,000 MSCs using a 21 hours' incubation time and $10 \mu \mathrm{g}$ USPIO/10 $0^{5} \mathrm{MSCs}$. At lower incubation times and doses of USPIO, significantly more cells were needed for detection. They confirmed their findings in ex vivo porcine myocardium by visualizing the USPIOlabeled MSCs.

USPIO-enhanced MRI appears well placed to provide future clinical research opportunities to detect and track stem cells following implantation. However, to enhance sensitivity, detection limits for stem cell number and administered iron concentration for a particular MRI scanner would need to be determined in advance.

\section{Apoptosis}

During apoptosis, or programmed cell death, the phospholipid phosphatidylserine is externalized on the cell surface. Annexin $\mathrm{V}$ is an endogenous protein that binds with high affinity to phosphatidylserine, and when conjugated to CLIO, has successfully been able to identify apoptotic cells in vitro using MRI. ${ }^{40}$ Sosnovik et $\mathrm{al}^{41}$ used this particle as a foundation to create a multimodal nanoparticle further conjugated to cyanine 5.5 dye - AnxCLIO-Cy5.5 - allowing co-identification with fluorescence microscopy and T2*-weighted MRI. In a murine model of ischemia reperfusion injury, a reduction in signal intensity on $\mathrm{T} 2 *$-weighted imaging was observed in reperfused regions with AnxCLIO-Cy5.5 but not with unlabeled CLIO-Cy5.5 suggesting uptake of AnxCLIO-Cy5.5 within the injured and apoptotic myocardium. The area of signal loss correlated with the region of contractile dysfunction seen on MRI, and subsequently with areas of increased fluorescence intensity on near infra-red fluorescence.

More recently, Sosnovik et al employed a similar approach in developing a novel dual-contrast molecular MRI probe capable of detecting cardiomyocyte apoptosis and necrosis in mice with ischemia reperfusion injury in vivo. ${ }^{42}$ Using the same AnxCLIO-Cy5.5 particle to identify cellular apoptosis on MRI 4-6 hours after ischemia-reperfusion injury, they also administered a novel magnetofluorescent gadolinium chelate with subsequent gadolinium delayed enhancement MRI to illustrate necrosis. Interestingly, they found that only $21 \%$ of apoptotic myocardium (accumulation of AnxCLIO-Cy5.5) had evidence of necrosis (defined by delayed gadolinium enhancement) suggesting that the majority of apoptotic cardiomyocytes remain viable 4-6 hours after ischemia-reperfusion injury. This was confirmed on fluores- cence microscopy and immunohistochemistry revealing the presence of large numbers of apoptotic, but potentially viable, cardiomyocytes in the "at-risk" area (AnxCLIO-Cy5.5 positive, Gd-DTPA-NBD negative areas). The authors remarked that strategies to salvage apoptotic, but not yet necrotic, myocardium should be explored now that these areas could be successfully imaged.

\section{Carotid atherosclerosis}

Carotid atherosclerosis and stroke are a major cause of disability and death. In the United States it is estimated that around 750,000 people become victims of stroke annually. ${ }^{43}$ Over one million Americans live with disabilities caused by stroke and $28 \%$ of strokes occur in people under the age of $65 .{ }^{44}$

Although the traditional imaging marker of disease burden has been stenosis, this is a poor marker of disease activity. It is now accepted that atherosclerosis is an inflammatory disorder ${ }^{45-47}$ involving recruitment of inflammatory cells into the lipid-rich plaque core. In atherosclerotic plaques, high macrophage density is a feature of vulnerability and correlates with risk of plaque rupture and clinical events. ${ }^{48}$ Modern techniques are now able to assess the composition and pathobiology of the arterial wall itself, and identification of vulnerable plaques at high risk of rupture is therefore key. USPIO-enhanced MRI is well placed to evaluate inflammation within plaques and will be discussed in this section.

Kresse et $\mathrm{a}^{49}$ first demonstrated USPIO uptake in atherosclerosis in vivo. They administered atherosclerotic rabbits custom-made iron oxide nanoparticles, visualizing these on MRI and confirming histologically with Prussian Blue staining. This study was uncontrolled and no attempt was made at quantitative analysis. Ruehm et a ${ }^{50}$ soon followed this with a more robust protocol. They administered USPIO to hyperlipidemic atherosclerotic rabbits and performed MRI scans at multiple time points. They noted extensive but patchy signal voids along the length of the aortic wall in atherosclerotic rabbits, but not in controls. Aortae were harvested for histological assessment and electron microscopy. Hyperlipidemic rabbits given USPIO showed extensive iron deposition in the subintima demonstrated by Prussian Blue staining, and on electron microscopy, iron inclusions were noted within plaque macrophages.

Hyafil et a ${ }^{51}$ examined hypercholesterolemic rabbits following iatrogenic aortic injury from balloon angioplasty and conducted MRI sequences at various time points following ferumoxtran-10 administration. At 5 days post-infusion, signal voids were seen in injured aortae on in vivo MRI, extending both inward into the lumen and outward into the periaortic region 
creating a "pseudostenosis" appearance. There was significantly more signal void in balloon-injured aortae compared to control aortae (uninjured aortic regions or rabbits administered saline instead of USPIO). Regions of signal void correlated with iron and RAM-11 staining (a rabbit macrophage marker), and a close correlation was also noted between the distribution of iron and RAM-11 staining. Extensive histological iron staining was seen in the balloon-injured aortae.

The first demonstration of the feasibility of atherosclerosis imaging using USPIO in humans was reported by Schmitz et $\mathrm{al}^{52}$ in a small study of patients with pelvic malignancy undergoing MRI for assessment of lymphatic metastasis using the USPIO ferumoxtran-10, which was at the time licensed for this indication. They retrospectively examined the aortic bifurcation and detected new focal areas of subintimal signal drop out in seven of 20 patients on T2*-weighted imaging that had not been present on the pre-USPIO images. The authors speculated that the presumed accumulation of subintimal USPIO was a consequence of dysfunctional endothelium and that this technique might function as an assay of endothelial integrity.

Kooi et $\mathrm{a}^{53}$ performed the first prospective human study evaluating atherosclerosis using USPIO (ferumoxtran-10) in a carotid endarterectomy model. Eleven patients scheduled to undergo carotid surgery following a symptomatic neurovascular event were imaged with $\mathrm{T} 2 *$-weighted MRI at three time points: pre-USPIO, 24 and 72 hours post-USPIO. Semi-quantitative image analysis was undertaken by calculating the relative signal intensity in quadrants with visible signal changes and comparing to reference tissue (skeletal muscle). Following surgery, carotid plaque was processed for immunohistochemical analysis and electron microscopy. Significant iron staining was noted in nine of ten plaques eligible for analysis, and regions co-localized with CD68 staining for macrophages. Electron microscopy confirmed this, demonstrating abundant USPIOsized intra-cytoplasmic inclusions within macrophages, which on energy-dispersive X-ray analysis were confirmed to be composed of iron. On magnetic resonance analysis, reduction in relative signal intensity was confined to areas of plaque that demonstrated positive histological iron staining. Maximum difference in pre- versus post-USPIO signal intensity occurred at 24 hours, with the 72 hour window failing to reach significance, suggesting that this time point was too late to image USPIO uptake.

Trivedi et $\mathrm{al}^{54}$ conducted a small study of eight patients scheduled for carotid endarterectomy, performing multisequence MRI at various time points (before, 24, 36, 48, and 72 hours after ferumoxtran-10 administration). Only segments of plaque staining positive for iron revealed signal void on MRI which was clearest on qualitative review of the T2*-weighted images at the 36 and 48 hour time points. This finding was replicated in the quantitative analysis (employing relative signal intensity) revealing greatest relative signal loss between 24 and 48 hours. Histological and electron microscopy analysis revealed most plaque sections demonstrated iron staining and that there was co-localization of iron and macrophage staining (MAC387), but there were regions with large populations of macrophages in the absence of iron staining. Electron microscopy confirmed the presence of USPIO-sized particles within macrophages.

In a subsequent study by the same authors, ${ }^{55} 30$ patients awaiting carotid endarterectomy for severe carotid stenosis were imaged before and 36 hours post-USPIO, in line with the findings of their previous work. MRI analysis was similar and more extensive histological validation was undertaken. Areas of reduced relative signal intensity were seen in 24/27 patients (three excluded for excessive artifact) that corresponded to USPIO/macrophage positive histology, and most samples $(23 / 30)$ revealed iron staining within the plaque. There was a significant correlation between the presence of USPIO (based upon change in relative signal intensity) on MRI and iron staining in plaques with focal but not diffuse distribution of signal void. Co-localization of iron staining with CD68 and MAC387 was seen but only modestly correlated (there were areas seen to stain for iron and not macrophages and vice versa), and in some sections there was extracellular iron staining around small neo-vessels, possibly indicating that these immature and leaky vessels may represent an entry port for USPIO.

The authors speculated that possible reasons for the weak correlation between iron and macrophage staining was a lack of sensitivity of Perls' stain for USPIO, delayed kinetics of ferumoxtran-10 in inflammatory tissue, and that macrophage subtypes might have differing uptake kinetics for USPIO. This latter theory has been given some experimental traction recently in a study which has shown that M1 and M2 macrophages take up USPIO at different rates. ${ }^{56}$ M1 polarized (pro-inflammatory/destructive) macrophages appear to take up relatively less USPIO than M2 polarized (anti-inflammatory/pre-healing) macrophages.

In 2006, Tang et $\mathrm{al}^{57}$ used a more objective analysis approach by calculating adjusted mean change in relative signal intensity. Instead of investigator selected regions of interest that are prone to bias, the mean change in relative signal intensity was calculated using multiple sample data points and a statistical model accounting for the correlation 
between signal loss in different quadrants within a slice and different slices within a plaque. They recruited 20 patients due to undergo carotid surgery and compared symptomatic to contralateral asymptomatic carotid artery plaques (mean stenosis $77 \%$ versus $46 \%$ respectively). They found that all symptomatic plaques demonstrated signal void and interestingly, 19/20 patients displayed regions of inflammation on the contralateral asymptomatic side. Symptomatic lesions did, however, have a greater percentage of positive quadrants $(72 \%$ versus $51 \%, P=0.007)$, and a significant reduction in adjusted mean relative signal intensity $(10.9 \% \pm 4.3 \%, P=0.02)$ that was not apparent on the contralateral side. They also observed that, as seen in previous studies, several asymptomatic "stable" plaques actually showed enhancement (signal increase) in the fibrous cap. The authors concluded that the results demonstrate the systemic nature of atherosclerotic inflammation and that this technique may aid risk-stratification of asymptomatic plaques in the future.

The notion of systemic inflammation in atherosclerosis was explored further in another study by Tang et al. ${ }^{58}$ Ten patients awaiting coronary artery bypass graft surgery for active coronary disease with incidental asymptomatic carotid stenosis $(>50 \%)$ were compared to ten individuals with similar severity of asymptomatic carotid stenosis but no known coronary artery disease. Plaques from patients awaiting coronary artery bypass graft surgery showed a significant mean signal intensity attenuation of $16.4 \%$ versus an increase (ie, enhancement) of $8.4 \%$ in the truly asymptomatic group making a true difference of $24.9 \%$ (95\% confidence interval $16.7 \%$ to $33.0 \% ; P<0.001)$ between groups. These data support the hypothesis that vascular inflammation is a systemic phenomenon measurable by USPIO accumulation and that signal enhancement may indicate a more stable plaque phenotype.

The same authors ${ }^{59}$ sought to clarify the relationship between traditional radiological markers of stroke risk and USPIO uptake in 71 individuals with asymptomatic carotid stenosis of varying severity. USPIO uptake on T2*W MRI was compared to luminal stenosis measurements of North American Symptomatic Carotid Endarterectomy Trial $(\text { NASCET) })^{60}$ and European Carotid Surgery Trial (ECST) $)^{61}$ methods, and normalized wall index. The group also made an attempt to model the USPIO data with respect to increasing stenosis severity. As with their previous work, no relationship was evident between either measurement of stenosis severity and plaque inflammation, and both are independent risk factors providing valuable and completely different pathological information. To further assess this, a prospective trial examining outcome data in patients stratified by USPIO uptake would be required.

The ATHEROMA (Atorvastatin THerapy: Effects on Reduction Of Macrophage Activity) trial was designed to evaluate the effects of statin therapy on carotid plaque inflammation. ${ }^{62}$ Forty-seven patients with carotid stenosis of greater than $40 \%$ who demonstrated plaque USPIO uptake were randomized to low $(10 \mathrm{mg})$ or high-dose $(80 \mathrm{mg})$ atorvastatin for 12 weeks with USPIO-enhanced MRI at 6 and 12 weeks. Twenty patients completed 12 weeks of treatment in each study arm. The primary end point was met with a significant reduction in USPIO uptake observed in the high-dose treatment arm at both 6 and 12 weeks. No such difference was evident in the low dose arm. Unsurprisingly, beneficial effects were noted on plasma lipid profiles but of greater interest was that microembolic load assessed by transcranial Doppler was significantly reduced. Furthermore, enhancement was seen in the fibrous cap and subintima in the high-dose but not the low-dose cohort, supporting previous observations that such enhancement may be associated with a more stable plaque phenotype. This study represents progress in developing a reliable imaging-based biomarker of atherosclerotic disease activity and inflammation. However, hard clinical outcomes are still lacking. Degnan et $\mathrm{a}^{63}$ performed a retrospective analysis of all participants screened for the ATHEROMA trial $(n=62)$ who received USPIO-enhanced MRI, and recorded the occurrence of stroke, transient ischaemic attack, MI or cardiovascular death during a median follow-up of 4 years. Although those who had events did appear to have greater mean change in relative signal intensity on MRI, there were small absolute differences that did not reach statistical significance $(P=0.07)$. When the population was divided into USPIO-positive or -negative plaque groups, survival curves did not diverge significantly. The authors conclude that their study was not powered to assess outcome but that there were perhaps encouraging signs emerging from the data.

\section{Abdominal aortic aneurysms}

Abdominal aortic aneurysms (AAAs) are defined as focal dilatations of the aorta, where aortic diameter is $>1.5$ times normal. They affect $5 \%$ of men over the age of 65 , and within this group cause $2 \%-3 \%$ of deaths annually. ${ }^{64,65}$ The vast majority of these deaths occur as a result of aortic rupture, which has a mortality rate of $65 \%-85 \%$ even in those reaching hospital alive. ${ }^{66,67}$ Currently, aneurysm diameter alone is the sole parameter used to stratify risk, but up to a fifth of ruptured 
AAAs have a diameter less than the $55 \mathrm{~mm}$ intervention threshold and many patients have larger aneurysms without symptoms. ${ }^{68,69}$ The implementation of aneurysm screening has reduced aneurysm-related mortality by up to $53 \%{ }^{64}$ but will increase the number of patients under long-term surveillance. Due to the unpredictability and non-linearity of aneurysm growth, improvements to the prediction of aneurysm rupture are required. Accordingly, there is a shift in focus from anatomical and morphological imaging to novel cellular and molecular techniques that evaluate lesion vulnerability on the basis of inflammatory and biological activity.

A prominent histological feature of AAAs is infiltration of $\mathrm{T}$ and $\mathrm{B}$ lymphocytes, macrophages, neutrophils, and natural killer cells. ${ }^{70-73}$ These inflammatory cells regulate the release of cytokines, resulting in the expression of cell adhesion molecules, protease production, and release of reactive oxygen species that in turn cause degradation of the extracellular matrix through alterations in the balance of matrix metalloproteinases and tissue inhibitors of matrix metalloproteinases..$^{74,75}$ In addition, inflammation and hypoxia lead to new vessel formation and loss of vascular smooth muscle cells, and it is these mechanisms that predispose to aortic dilatation and increased risk of AAA rupture. Inflammation within the aortic wall is often focally distributed and it is these hotspots of pathological activity that have been the focus of much investigation.

Howarth et al were the first to report USPIO uptake in AAA in man. They described simultaneous USPIO uptake within atheroma of both the internal carotid artery and a $56 \mathrm{~mm}$, asymptomatic AAA. ${ }^{76}$ Truijers et al later described USPIO (ferumoxtran-10) uptake in the wall of a small number of asymptomatic AAAs $(n=6) .{ }^{77}$ They found significantly more USPIO-positive quadrants in aneurysm patients than in age-matched controls, and the patient with the largest number of USPIO-positive quadrants had the largest diameter aneurysm although there was a wide variation in USPIO uptake. A subsequent pilot feasibility study by Sadat et al $(n=14)$ quantified USPIO uptake in the AAA wall by using calculated T2 and T2* values. ${ }^{78}$ They found significant correlation between imaging sequences pre- and 36 hours post-USPIO infusion, and both imaging methods were able to detect the presence of USPIO uptake.

Our group evaluated the potential for this technique to predict aneurysm expansion. Twenty-nine asymptomatic patients with AAAs underwent baseline MRI scans followed by intravenous administration of USPIO and repeat MRI scans 24-36 hours later. A similar technique was used to assess iron accumulation; the percentage change in T2* value was calculated following USPIO administration, but additionally, a threshold of significance for change was applied. By applying this threshold based on repeatability data for calculation of the T2* value in vascular tissue of subjects not receiving USPIO, we were able to distinguish changes in $\mathrm{T} 2 *$ associated with USPIO accumulation from artifact. All patients demonstrated non-specific peri-luminal USPIO accumulation, likely reflecting USPIO uptake in the fresh, highly cellular component of the thrombus. Thrombus within AAAs is structurally complex and is known to consist of a continuous network of interconnected canaliculi. ${ }^{79}$ This network may support transportation of inflammatory cells from lumen into the thrombus, and further into aortic wall as seen in some patients. Importantly, despite similar aneurysm sizes, it is these patients with mural USPIO uptake who had evidence of aneurysm growth rates three-fold higher (6.6 $\mathrm{mm} /$ year) than patients with no mural USPIO uptake, suggesting that this technique may be used to identify patients at greater risk of aneurysm growth. Aortic tissue was collected from patients undergoing open surgical aneurysm repair and histological analysis confirmed co-localization

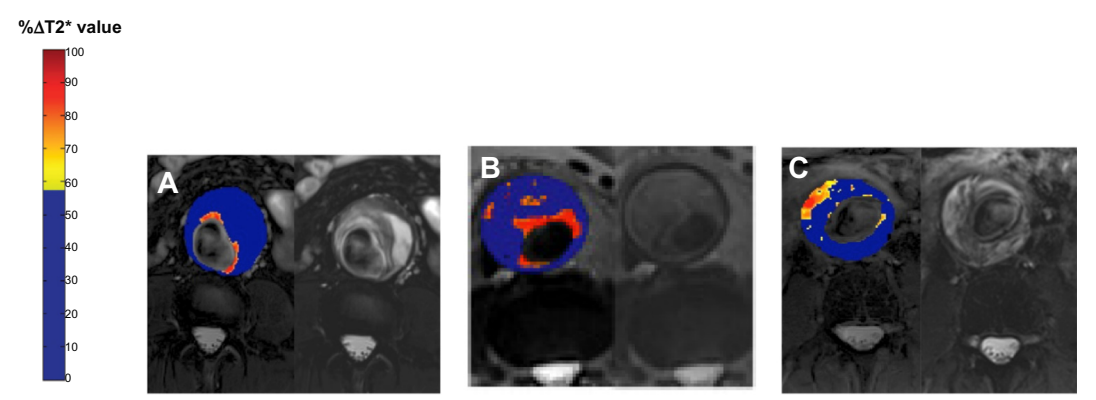

Figure 3 T2*-weighted color maps showing alongside the corresponding T2-weighted anatomic images from patients with abdominal aortic aneurysms (AAA). Notes: The color scale represents the magnitude of the change in $T 2^{*}$ value, with blue indicating minimal change and red indicating a large change in $T 2^{*}$ value using a previously defined threshold above which changes in T2* value can be attributed to ultra-small superparamagnetic particles of iron oxide (USPIO) accumulation. Only changes in T2* value above the threshold are presented on the graduated (yellow-red) color scale; data below the threshold appear blue. (A) shows a change in T2* value confined to the peri-luminal area; (B) diffuse patchy changes in T2* throughout the intraluminal thrombus but no distinct focal area of USPIO uptake affecting the aortic wall; and (C) discrete focal area of USPIO uptake involving the wall of the AAA that is distinct from the peri-luminal region. 


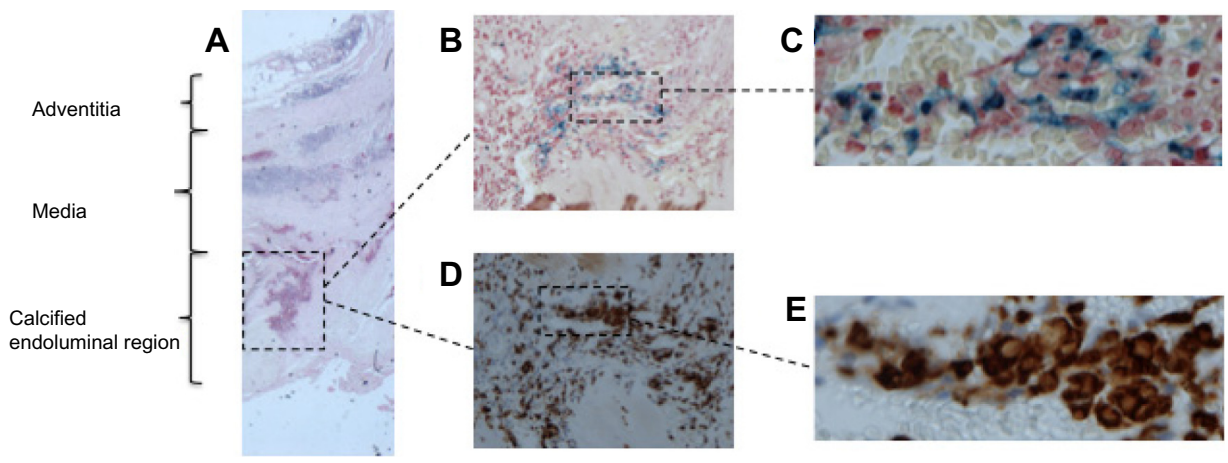

Figure 4 Histology from an abdominal aortic aneurysm wall.

Notes: (A) Hematoxylin and eosin staining at $\times 20$ - inflammatory cell infiltrates seen in the endoluminal region with focal calcified atheroma. (B) Perls' stain for iron at $\times 100$ and (C) $\times 600$ showing the presence of iron (blue). (D) CD68 staining for macrophages (brown) at $\times 100$ and (E) $\times 600$ showing macrophages colocalizing with iron stain.

of USPIO with CD68 staining for macrophages. ${ }^{80}$ An observational surveillance study is currently underway in our center ( $\mathrm{MA}^{3} \mathrm{RS}$ study) examining whether USPIOenhanced MRI is able to predict the likelihood of rupture or need for surgery (Figures 3 and 4).

\section{Conclusion and future applications}

USPIO can detect inflammation in cardiac, carotid, and aortic disease, whilst providing targeted imaging in stem cell therapy and apoptosis. It is a technique that holds major promise for translation into the clinic.

The development of iron nanoparticle-enhanced MRI has been greatly affected by the discontinuation of several agents in recent years. Ferumoxtran-10 $\left(\right.$ Sinerem $\left.^{\circledR}\right)$, was discontinued due to a high false-positive rate of detecting lymph node metastasis in patients with prostate cancer. ${ }^{81}$ NC100500 (Clariscan ${ }^{\mathrm{TM}}$ ) was discontinued over concerns regarding long-term liver retention, ${ }^{82}$ and the larger SPIO ferumoxides (Feridex ${ }^{\circledR}$ in United States, Endorem ${ }^{\mathrm{TM}}$ in Europe), and ferucarbotran (Resovist ${ }^{\mathbb{R}}$ ) have also been discontinued; the latter two agents were the only two clinically approved SPIO agents for MRI. At present there is only one iron oxide particle preparation available for clinical use: ferumoxytol (Rienso $^{\circledR}$; Takeda Italia S.p.A, Rome, Italy), a 17-30 nm USPIO. Rienso is marketed for the intravenous treatment of iron deficiency anemia in adult patients with chronic kidney disease, but is currently not licensed for imaging.

There are several clear opportunities for the developments of USPIO-enhanced MRI as a clinical research tool and our group is using ferumoxytol-enhanced MRI successfully in several clinical research studies. We are attempting to characterize myocardial inflammation following MI in humans: using the assessment of cellular myocardial inflammation as a biomarker for therapeutic interventions. We are also piloting USPIO-enhanced MRI as a diagnostic test in other inflammatory myocardial diseases such as sarcoidosis and myocarditis. At the same time, we are exploring whether USPIO-enhanced MRI can identify patients with high-risk atherosclerotic plaques and AAAs who require early medical or surgical intervention. There are also clear opportunities for USPIO-enhanced MRI in the development of stem cell therapy.

It is difficult to predict where USPIO-enhanced MRI will make a breakthrough into clinical practice but there are clear applications in a range of cardiovascular and noncardiovascular diseases. This exciting imaging technique has great potential and results of research studies in coming years will determine its place in the clinic.

\section{Disclosure}

The British Heart Foundation (FS/07/060, FS/12/83/29781, PG/12/8/29371), National Institutes for Health Research (Efficacy and Mechanism Evaluation, 11/20/03), Medical Research Council (96182) and Chest Heart and Stroke Scotland (r11/A135) and Chief Scientist Office (ETM/266) have provided grants supporting work in this field at our center carried out by CGS, ATV, OMBM, SRA, JMJR, PAH, and DEN. DEN is supported by the British Heart Foundation (CH/09/002). The authors have no other conflicts of interest in this work.

\section{References}

1. Wang XY, Hussain SM, Krestin GP. Superparamagnetic iron oxide contrast agents: physicochemical characteristics and applications in MR imaging. Eur Radiol. 2001;11(11):2319-2331.

2. Allkemper T, Bremer C, Matuszewski L, Ebert W, Reimer P. Contrastenhanced blood-pool MR angiography with optimized iron oxides: effect of size and dose on vascular contrast enhancement in rabbits. Radiology. 2002;223(2):432-438. 
3. Mandeville JB, Moore J, Chesler DA, Garrido L, Weissleder R, Weisskoff RM. Dynamic liver imaging with iron oxide agents: effects of size and biodistribution on contrast. Magn Reson Med. 1997;37(6):885-890.

4. McLachlan SJ, Morris MR, Lucas MA, et al. Phase I clinical evaluation of a new iron oxide MR contrast agent. J Magn Reson Imaging. 1994;4(3):301-307.

5. Clément O, Siauve N, Cuénod CA, Frija G. Liver imaging with ferumoxides (Feridex): fundamentals, controversies, and practical aspects. Top Magn Reson Imaging. 1998;9(3):167-182.

6. Mahmoudi M, Simchi A, Imani M, Milani AS, Stroeve P. An in vitro study of bare and poly(ethylene glycol)-co-fumarate-coated superparamagnetic iron oxide nanoparticles: a new toxicity identification procedure. Nanotechnology. 2009;20(22):225104.

7. Mahmoudi M, Simchi A, Imani M, et al. A new approach for the in vitro identification of the cytotoxicity of superparamagnetic iron oxide nanoparticles. Colloids Surf B Biointerfaces. 2010;75(1):300-309.

8. Berry CC, Wells S, Charles S, Aitchison G, Curtis A. Cell response to dextran-derivatised iron oxide nanoparticles post internalisation. Biomaterials. 2004;25(23):5405-5413.

9. Berry CC, Wells S, Charles S, Curtis AS. Dextran and albumin derivatised iron oxide nanoparticles: influence on fibroblasts in vitro. Biomaterials. 2003;24(25):4551-4557.

10. Wang YX. Superparamagnetic iron oxide based MRI contrast agents: Current status of clinical application. Quant Imaging Med Surg. 2011;1(1):35-40.

11. Stillman AE, Wilke N, Jerosch-Herold M. Use of an intravascular T1 contrast agent to improve MR cine myocardial-blood pool definition in man. J Magn Reson Imaging. 1997;7(4):765-767.

12. Taylor AM, Panting JR, Keegan J, et al. Use of the intravascular contrast agent NC100150 injection in spin-echo and gradient-echo imaging of the heart. J Cardiovasc Magn Reson. 1999;1(1):23-32.

13. Paetsch I, Thiele H, Schnackenburg B, et al. Improved functional cardiac MR imaging using the intravascular contrast agent CLARISCAN. Int J Cardiovasc Imaging. 2003;19(4):337-343.

14. Bunce NH, Moon JC, Bellenger NG, et al. Improved cine cardiovascular magnetic resonance using Clariscan (NC100150 injection). J Cardiovasc Magn Reson. 2001;3(4):303-310.

15. Amano Y, Herfkens RJ, Shifrin RY, Alley MT, Pelc NJ. Threedimensional cardiac cine magnetic resonance imaging with an ultrasmall superparamagnetic iron oxide blood pool agent (NC100150). J Magn Reson Imaging. 2000;11(2):81-86.

16. Johansson LO, Nolan MM, Taniuchi M, Fischer SE, Wickline SA, Lorenz $\mathrm{CH}$. High-resolution magnetic resonance coronary angiography of the entire heart using a new blood-pool agent, NC100150 injection: comparison with invasive $\mathrm{x}$-ray angiography in pigs. $J$ Cardiovasc Magn Reson. 1999;1(2):139-143.

17. Sandstede JJ, Pabst T, Wacker C, et al. Breath-hold 3D MR coronary angiography with a new intravascular contrast agent (feruglose) - first clinical experiences. Magn Reson Imaging. 2001;19(2):201-205.

18. Klein C, Schalla S, Schnackenburg B, et al. Improvement of image quality of non-invasive coronary artery imaging with magnetic resonance by the use of the intravascular contrast agent Clariscan (NC100150 injection) in patients with coronary artery disease. J Magn Reson Imaging. 2003;17(6):656-662.

19. Kroft LJM, Doornbos J, van der Geest RJ, van der Laarse A, van der Meulen H, de Roos A. Ultrasmall superparamagnetic particles of iron oxide (USPIO) MR imaging of infarcted myocardium in pigs. Magn Reson Imaging. 1998;16(7):755-763.

20. Bjerner T, Ericsson A, Wikström G, et al. Evaluation of nonperfused myocardial ischemia with MRI and an intravascular USPIO contrast agent in an ex vivo pig model. J Magn Reson Imaging. 2000;12(6):866-872.

21. Krombach GA, Wendland MF, Higgins CB, Saeed M. MR imaging of spatial extent of microvascular injury in reperfused ischemically injured rat myocardium: value of blood pool ultrasmall superparamagnetic particles of iron oxide. Radiology. 2002;225(2):479-486.
22. Cooper LT, Baughman KL, Feldman AM, et al. The role of endomyocardial biopsy in the management of cardiovascular disease A scientific statement from the American Heart Association, the American College of Cardiology, and the European Society of Cardiology endorsed by the Heart Failure Society of America and the Heart Failure Association of the European Society of Cardiology. J Am Coll Cardiol. 2007;50(19):1914-1931.

23. Hertz MI, Aurora P, Christie JD, et al. Scientific registry of the International Society for Heart and Lung Transplantation: introduction to the 2009 annual reports. J Heart Lung Transplant. 2009;28(10): 989-992.

24. Kanno S, Wu YJ, Lee PC, Dodd SJ, et al. Macrophage accumulation associated with rat cardiac allograft rejection detected by magnetic resonance imaging with ultrasmall superparamagnetic iron oxide particles. Circulation. 2001;104(8):934-938.

25. Johansson L, Johnsson C, Penno E, Bjornerud A, Ahlstrom H. Acute cardiac transplant rejection: detection and grading with MR imaging with a blood pool contrast agent experimental study in the rat. Radiology. 2002;225(1):97-103.

26. Penno E, Johnsson C, Johansson L, Ahlström H. Comparison of ultrasmall superparamagnetic iron oxide particles and low molecular weight contrast agents to detect rejecting transplanted hearts with magnetic resonance imaging. Invest Radiol. 2005;40(10): 648-654.

27. Wu YL, Ye Q, Sato K, Foley LM, Hitchens TK, Ho C. Noninvasive evaluation of cardiac allograft rejection by cellular and functional cardiac magnetic resonance. JACC Cardiovasc Imaging. 2009;2(6):731-741.

28. Penno E, Johnsson C, Johansson L, Ahlstrom H. Macrophage uptake of ultra-small iron oxide particles for magnetic resonance imaging in experimental acute cardiac transplant rejection. Acta Radiol. 2009;47(3):264-271.

29. McAteer MA, Akhtar AM, Zur Muhlen von C, Choudhury RP. An approach to molecular imaging of atherosclerosis, thrombosis, and vascular inflammation using microparticles of iron oxide. Atherosclerosis. 2010;209(1):18-27.

30. Guo Y, Chen W, Wang W, et al. Simultaneous diagnosis and gene therapy of immuno-rejection in rat allogeneic heart transplantation model using a T-cell-targeted theranostic nanosystem. ACS Nano. 2012;6(12):10646-10657.

31. Wunderbaldinger P, Josephson L, Weissleder R. Crosslinked iron oxides (CLIO): a new platform for the development of targeted MR contrast agents. Acad Radiol. 2002;9 Suppl 2:S304-S306.

32. Sosnovik DE, Nahrendorf M, Deliolanis N, et al. Fluorescence tomography and magnetic resonance imaging of myocardial macrophage infiltration in infarcted myocardium in vivo. Circulation. 2007;115(11):1384-1391.

33. Alam SR, Shah AS, Richards J, et al. Ultrasmall superparamagnetic particles of iron oxide in patients with acute myocardial infarction: early clinical experience. Circ Cardiovasc Imaging. 2012;5(5):559-565.

34. Yilmaz A, Dengler MA, van der Kuip H, et al. Imaging of myocardial infarction using ultrasmall superparamagnetic iron oxide nanoparticles: a human study using a multi-parametric cardiovascular magnetic resonance imaging approach. Eur Heart J. 2013;34(6): 462-475.

35. Grieve SM, Lønborg J, Mazhar J, et al. Cardiac magnetic resonance imaging of rapid VCAM-1 up-regulation in myocardial ischemiareperfusion injury. Eur Biophys J. 2012;42(1):61-70.

36. Küstermann E, Roell W, Breitbach M, et al. Stem cell implantation in ischemic mouse heart: a high-resolution magnetic resonance imaging investigation. NMR Biomed. 2005;18(6):362-370.

37. Chapon C, Jackson JS, Aboagye EO, Herlihy AH, Jones WA, Bhakoo KK. An in vivo multimodal imaging study using MRI and PET of stem cell transplantation after myocardial infarction in rats. Mol Imaging Biol. 2009;11(1):31-38.

38. Ma GS, Qi CM, Liu NF, et al. Efficiently tracking of stem cells in vivo using different kinds of superparamagnetic iron oxide in swine with myocardial infarction. Chin Med J (Engl). 2011;124(8): 1199-1204. 
39. Mathiasen AB, Hansen L, Friis T, Thomsen C, Bhakoo K, Kastrup J. Optimal labeling dose, labeling time, and magnetic resonance imaging detection limits of ultrasmall superparamagnetic ironoxide nanoparticle labeled mesenchymal stromal cells. Stem Cells Int. 2013;2013:353105.

40. Schellenberger EA, Högemann D, Josephson L, Weissleder R. Annexin V-CLIO: a nanoparticle for detecting apoptosis by MRI. Acad Radiol. 2002;9 Suppl 2:S310-S311.

41. Sosnovik DE, Schellenberger EA, Nahrendorf M, et al. Magnetic resonance imaging of cardiomyocyte apoptosis with a novel magneto-optical nanoparticle. Magn Reson Med. 2005;54(3): $718-724$.

42. Sosnovik DE, Garanger E, Aikawa E, et al. Molecular MRI of cardiomyocyte apoptosis with simultaneous delayed-enhancement MRI distinguishes apoptotic and necrotic myocytes in vivo potential for midmyocardial salvage in acute ischemia. Circ Cardiovasc Imaging. 2009;2(6):460-467.

43. Sobieszczyk P, Beckman J. Carotid artery disease. Circulation. 2006;114(7):e244-e247.

44. Stroke [webpage on the Internet]. Fairfax: Society of Interventional Radiology; 2014. Available from: http://www.sirweb.org/patients/ stroke/. Accessed August 24, 2014.

45. Ross R. Atherosclerosis - An inflammatory disease. $N$ Engl J Med. 1999;340(2):115-126.

46. Libby P. Inflammation in atherosclerosis. Nature. 2002;420(6917): 868-874.

47. Libby P. Inflammation in Atherosclerosis. Arterioscler Thromb Vasc Biol. 2012;32(9):2045-2051.

48. Naghavi M, Libby P, Falk E, et al. From vulnerable plaque to vulnerable patient: a call for new definitions and risk assessment strategies: part II. Circulation. 2003;108(15):1772-1778.

49. Kresse M, Wagner S, Thode K, Dinkelborg L. MR plaque imaging using superparamagnetic iron oxide particles. Proc ISMRM. 1998.

50. Ruehm SG, Corot C, Vogt P, Kolb S, Debatin JF. Magnetic resonance imaging of atherosclerotic plaque with ultrasmall superparamagnetic particles of iron oxide in hyperlipidemic rabbits. Circulation. 2001;103(3):415-422.

51. Hyafil F, Laissy JP, Mazighi M, et al. Ferumoxtran-10-enhanced MRI of the hypercholesterolemic rabbit aorta: relationship between signal loss and macrophage infiltration. Arterioscler Thromb Vasc Biol. 2006;26(1):176-181.

52. Schmitz SA, Taupitz M, Wagner S, Wolf KJ, Beyersdorff D, Hamm B. Magnetic resonance imaging of atherosclerotic plaques using superparamagnetic iron oxide particles. J Magn Reson Imaging. 2001; 14(4):355-361.

53. Kooi ME, Cappendijk VC, Cleutjens KB, et al. Accumulation of ultrasmall superparamagnetic particles of iron oxide in human atherosclerotic plaques can be detected by in vivo magnetic resonance imaging. Circulation. 2003;107(19): 2453-2458.

54. Trivedi RA, U-King-Im JM, Graves MJ, et al. In vivo detection of macrophages in human carotid atheroma: temporal dependence of ultrasmall superparamagnetic particles of iron oxide-enhanced MRI. Stroke. 2004;35(7):1631-1635

55. Trivedi RA, Mallawarachi C, U-King-Im JM, Graves MJ, et al. Identifying inflamed carotid plaques using in vivo USPIO-enhanced MR imaging to label plaque macrophages. Arterioscler Thromb Vasc Biol. 2006;26(7):1601-1606.

56. Satomi T, Ogawa M, Mori I, et al. Comparison of contrast agents for atherosclerosis imaging using cultured macrophages: FDG versus ultrasmall superparamagnetic iron oxide. J Nucl Med. 2013;54(6): 999-1004.

57. Tang T, Howarth SP, Miller SR, et al. Assessment of inflammatory burden contralateral to the symptomatic carotid stenosis using highresolution ultrasmall, superparamagnetic iron oxide-enhanced MRI. Stroke. 2006;37(9):2266-2270.
58. Tang TY, Howarth SP, Miller SR, et al. Comparison of the inflammatory burden of truly asymptomatic carotid atheroma with atherosclerotic plaques in patients with asymptomatic carotid stenosis undergoing coronary artery bypass grafting: an ultrasmall superparamagnetic iron oxide enhanced magnetic resonance study. Eur J Vasc Endovasc Surg. 2008;35(4):392-398.

59. Tang TY, Howarth SP, Miller SR, et al. Correlation of carotid atheromatous plaque inflammation using USPIO-enhanced MR imaging with degree of luminal stenosis. Stroke. 2008;39(7):2144-2147.

60. North American Symptomatic Carotid Endarterectomy Trial Collaborators. Beneficial effect of carotid endarterectomy in symptomatic patients with high-grade carotid stenosis. $N$ Engl J Med. 1991;325(7): $445-453$.

61. [No authors listed] Randomised trial of endarterectomy for recently symptomatic carotid stenosis: final results of the MRC European Carotid Surgery Trial (ECST). Lancet. 1998;351(9113):1379-1387.

62. Tang TY, Howarth SP, Miller SR, et al. The ATHEROMA (Atorvastatin Therapy: Effects on Reduction of Macrophage Activity) study evaluation using ultrasmall superparamagnetic iron oxide-enhanced magnetic resonance imaging in carotid disease. J Am Coll Cardio. 2009;53(22):2039-2050.

63. Degnan AJ, Patterson AJ, Tang TY, Howarth SPS, Gillard JH. Evaluation of ultrasmall superparamagnetic iron oxide-enhanced MRI of carotid atherosclerosis to assess risk of cerebrovascular and cardiovascular events: follow-up of the ATHEROMA trial. Cerebrovasc Dis. 2012;34(2):169-173.

64. Scott R. The Multicentre Aneurysm Screening Study (MASS) into the effect of abdominal aortic aneurysm screening on mortality in men: a randomised controlled trial. Lancet. 2002;360(9345):1531-1539.

65. Gillum RF. Epidemiology of aortic aneurysm in the United States. 1995;48(11):1289-1298.

66. Basnyat PS, Biffin AHB, Moseley LG, Hedges AR, Lewis MH. Mortality from ruptured abdominal aortic aneurysm in Wales. British J of Surgery. 1999;86:765-770.

67. Johnston KW. Ruptured abdominal aortic aneurysm: six-year follow-up results of a multicenter prospective study. Canadian Society for Vascular Surgery Aneurysm Study Group. J Vasc Surg. 1994;19(5): 888-900.

68. Darling RC, Messina CR, Brewster DC, Ottinger LW. Autopsy study of unoperated abdominal aortic aneurysms. The case for early resection. Circulation. 1977;56(3 Suppl):II161-II164.

69. Powell JT, Brown LC, Greenhalgh RM, Thompson SG. The rupture rate of large abdominal aortic aneurysms: is this modified by anatomical suitability for endovascular repair? Ann Surg. 2008;247(1): 173-179.

70. Eliason JL, Hannawa KK, Ailawadi G, et al. Neutrophil depletion inhibits experimental abdominal aortic aneurysm formation. Circulation. 2005;112(2):232-240.

71. Reeps C, Pelisek J, Seidl S, et al. Inflammatory infiltrates and neovessels are relevant sources of MMPs in abdominal aortic aneurysm wall. Pathobiology. 2009;76(5):243-252.

72. Houard X, Ollivier V, Louedec L, Michel JB, Back M. Differential inflammatory activity across human abdominal aortic aneurysms reveals neutrophil-derived leukotriene B4 as a major chemotactic factor released from the intraluminal thrombus. FASEB J. 2009;23(5): 1376-1383.

73. Ocana E, Bohórquez JC, Pérez-Requena J, Brieva JA, Rodríguez C. Characterisation of T and B lymphocytes infiltrating abdominal aortic aneurysms. Atherosclerosis. 2003;170(1):39-48.

74. Thompson MM, Wills A, McDermott E, Crowther M, Brindle N, Bell PR. An in vitro model of aneurysmal disease: effect of leukocyte infiltration and shear stress on MMP production within the arterial wall. Ann N Y Acad Sci. 1996;800:270-273.

75. Anidjar S, Salzmann JL, Gentric D, Lagneau P, Camilleri JP, Michel JB. Elastase-induced experimental aneurysms in rats. Circulation. 1990;82(3):973-981. 
76. Howarth SP, Tang TY, Graves MJ, et al. Non-invasive MR imaging of inflammation in a patient with both asymptomatic carotid atheroma and an abdominal aortic aneurysm: a case report. Ann Surg Innov Res. 2007; $1: 4$.

77. Truijers M, Fütterer JJ, Takahashi S, Heesakkers RA, Blankensteijn JD, Barentsz JO. In vivo imaging of the aneurysm wall with MRI and a macrophage-specific contrast agent. AJR Roentgenol. 2009;193(5): W437-W441.

78. Sadat U, Taviani V, Patterson AJ, et al. Ultrasmall superparamagnetic iron oxide-enhanced magnetic resonance imaging of abdominal aortic aneurysms - A feasibility study. Eur J Vasc Endovasc Surg. 2011;41(2):167-174
79. Adolph R, Vorp DA, Steed DL, Webster MW, Kameneva MV, Watkins SC. Cellular content and permeability of intraluminal thrombus in abdominal aortic aneurysm. J Vasc Surg. 1997;25(5):916-926.

80. Richards JM, Semple SI, MacGillivray TJ, et al. Abdominal aortic aneurysm growth predicted by uptake of ultrasmall superparamagnetic particles of iron oxide: a pilot study. Circ Cardiovasc Imaging. 2011;4(3):274-281.

81. Heesakkers RA, Jager GJ, Hövels AM, et al. Prostate cancer: detection of lymph node metastases outside the routine surgical area with ferumoxtran-10-enhanced MR imaging. Radiology. 2009;251(2):408-414.

82. Ho V, Reddy GP. Cardiovascular Imaging. Elsevier Health Sciences; 2010.

\section{Publish your work in this journal}

Journal of Vascular Diagnostics is an international, peer-reviewed journal of diagnostics, focusing on non invasive vascular investigation methods involved in the evaluation of vascular diseases. The journal is committed to the rapid publication in the fields of vascular diseases. Original research, review, case reports, expert opinion and commentaries
Dovepress

are all considered for publication. The manuscript management system is completely online and includes a very quick and fair peer-review system, which is all easy to use. Visit http://www.dovepress.com/testimonials.php to read real quotes from published authors.

Submit your manuscript here: http://www.dovepress.com/journal-of-vascular-diagnostics-journal 


\title{
Ferumoxytol-enhanced magnetic resonance imaging methodology and normal values at 1.5 and $3 \mathrm{~T}$
}

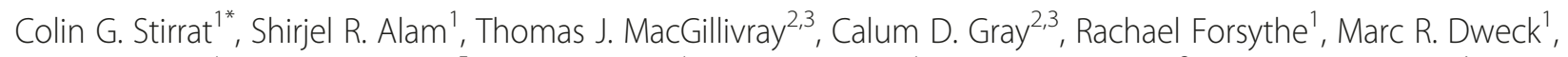
John R. Payne", Sanjay K. Prasad ${ }^{5}$, Mark C. Petrie ${ }^{4}$, Roy S. Gardner ${ }^{4}$, Saeed Mirsadraee ${ }^{2}$, Peter A. Henriksen?', David E. Newby ${ }^{1,2}$ and Scott I. K. Semple $e^{1,2}$

\begin{abstract}
Background: Ultrasmall superparamagnetic particles of iron oxide (USPIO)-enhanced magnetic resonance imaging (MRI) can detect tissue-resident macrophage activity and identify cellular inflammation. Clinical studies using this technique are now emerging. We aimed to report a range of normal $R 2^{*}$ values at 1.5 and $3 \mathrm{~T}$ in the myocardium and other tissues following ferumoxytol administration, outline the methodology used and suggest solutions to commonly encountered analysis problems.

Methods: Twenty volunteers were recruited: 10 imaged each at $1.5 \mathrm{~T}$ and $3 \mathrm{~T} . \mathrm{T} 2^{*}$ and late gadolinium enhanced (LGE) MRI was conducted at baseline with further T2* imaging conducted approximately $24 \mathrm{~h}$ after USPIO infusion (ferumoxytol, $4 \mathrm{mg} / \mathrm{kg}$ ). Regions of interest were selected in the myocardium and compared to other tissues.

Results: Following administration, USPIO was detected by changes in R2* from baseline $\left(1 / T 2^{*}\right)$ at $24 \mathrm{~h}$ in myocardium, skeletal muscle, kidney, liver, spleen and blood at 1.5 T, and myocardium, kidney, liver, spleen, blood and bone at $3 \mathrm{~T}$ $(p<0.05$ for all). Myocardial changes in R2* due to USPIO were $26.5 \pm 7.3 \mathrm{~s}-1$ at $1.5 \mathrm{~T}$, and $37.2 \pm 9.6 \mathrm{~s}-1$ at $3 \mathrm{~T}(p<0.0001$ for both). Tissues showing greatest ferumoxytol enhancement were the reticuloendothelial system: the liver, spleen and bone marrow $(216.3 \pm 32.6 s-1,336.3 \pm 60.3 s-1,69.9 \pm 79.9 s-1 ; p<0.0001, p<0.0001, p=n s$ respectively at $1.5 \mathrm{~T}$, and $275.6 \pm 69.9 s-1,463.9 \pm 136.7 s-1,417.9 \pm 370.3 s-1 ; p<0.0001, p<0.0001, p<0.01$ respectively at $3 \mathrm{~T})$.
\end{abstract}

Conclusion: Ferumoxytol-enhanced MRI is feasible at both $1.5 \mathrm{~T}$ and $3 \mathrm{~T}$. Careful data selection and dose administration, along with refinements to echo-time acquisition, post-processing and analysis techniques are essential to ensure reliable and robust quantification of tissue enhancement.

Trial registration: ClinicalTrials.gov Identifier - NCT02319278. Registered 03.12.2014.

Keywords: Cardiac, MRI, Inflammation, USPIO

\section{Background}

Iron oxide nanoparticles are a class of magnetic resonance imaging (MRI) contrast agents that are generating interest as a method of detecting tissue inflammation. Historically, these nanoparticles were initially used for gastrointestinal, reticuloendothelial system and lymph node imaging [1-3], and subsequently in hepatic and cardiac imaging [4-7]. Recently however, it is in their

\footnotetext{
*Correspondence: colin.stirrat@ed.ac.uk

${ }^{1}$ British Heart Foundation/University Centre for Cardiovascular Science, University of Edinburgh, Edinburgh, UK

Full list of author information is available at the end of the article
}

use as an MRI contrast agent for detecting tissueresident macrophages that clinical applications are now emerging [8-15].

T2* MRI has been successfully used for over a decade in diagnosing and grading severity of iron accumulation in transfusion-dependent thalassaemia major, and has been instrumental in guiding therapy that improves prognosis, and allows serial disease monitoring [16, 17]. T2* MRI in the assessment of iron accumulation is easily quantifiable, well validated, highly reproducible, clinically robust, and is achievable in a single breath hold [18-22]. 


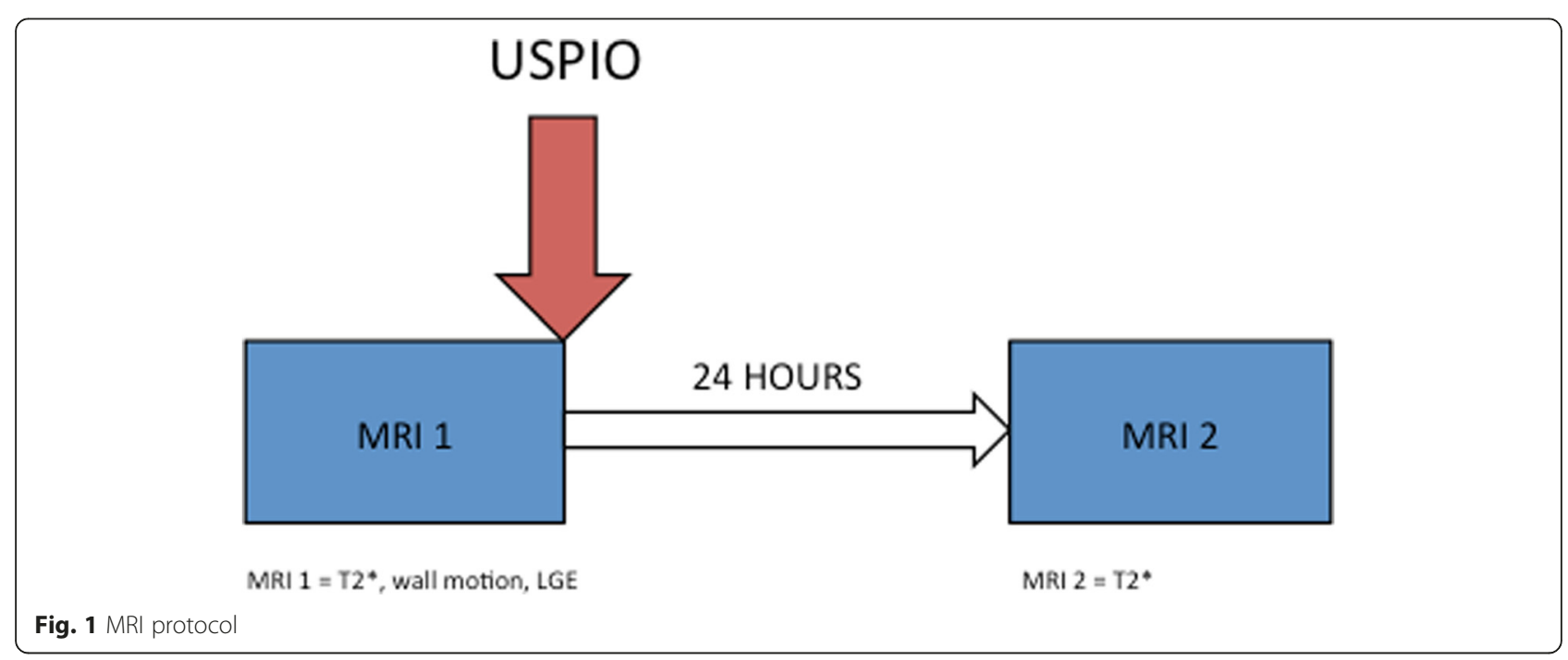

Ultrasmall superparamagnetic particles of iron oxide (USPIO) consist of an iron oxide core surrounded by a carbohydrate or polymer coating. These particles can extravasate through damaged capillaries, where they are engulfed and concentrated by tissue-resident macrophages [23]. Gradient echo T2*-weighted $(\mathrm{T} 2 * \mathrm{~W})$ sequences are highly sensitive to magnetic field inhomogeneities such as susceptibility artifacts due to the presence of iron, including USPIO. Accumulation of USPIOs in macrophages can be quantified and visualized using T2*W MRI $[8,9]$ and calculation of, and observing the reduction in, $\mathrm{T} 2$ * relaxation time due to the presence of iron. Thus USPIOenhanced MRI can detect tissue-resident macrophage activity and identify localized cellular inflammation within tissues.

In this present study we aimed to observe and quantify the distribution ferumoxytol enhancement following intravenous administration at 1.5 and $3 \mathrm{~T}$ MRI and establish a range of normal values for healthy myocardium and other tissue. We also aimed to develop our methodology and describe commonly encountered problems in T2* image analysis of USPIO.

\section{Methods}

This was an open-label observational multi-centre cohort study using human volunteers recruited as part of a larger trial, recruiting patients with cardiac inflammation. The study was performed in accordance with the declaration of Helsinki, the approval of the Scotland A research ethics committee, and the written informed consent of all participants.

\section{Subjects}

Participants were aged over 18 years of age. Exclusion criteria were contraindication to MRI or ferumoxytol infusion, any systemic inflammatory comorbidity (eg rheumatoid arthritis), renal failure (estimated glomerular filtration rate $<30 \mathrm{~mL} / \mathrm{min}$ ), pregnancy, breastfeeding and women of child-bearing age not ensuring reliable contraception.

\section{Magnetic resonance imaging}

MRI was performed using $3 \mathrm{~T}$ and $1.5 \mathrm{~T}$ scanners (Magnetom Verio and Avanto respectively, Siemens Healthcare $\mathrm{GmbH}$, Erlangen, Germany), with dedicated cardiac array coils. All images were acquired using electrocardiogramgated breath-hold imaging. Routine steady state free precession (TrueFISP) sequences were used to acquire long-axis and short-axis images of the heart. Standard cardiac slice widths (6-mm width with 4-mm gap) and 8 echo times (2.1-17.1 ms range) with matrix size of $256 \times 115$ were acquired in order to generate $\mathrm{T} 2 *$ maps. The in-plane resolution differed as required for larger or smaller subjects; generally, a field of view of $400 \times 300 \mathrm{~mm}$ was used with an in-plane resolution of $2.6 \times 1.6 \mathrm{~mm}$. T2* relaxation maps were generated before and approximately $24 \mathrm{~h}$ after administration of USPIO.

Immediately after the baseline $\mathrm{T}_{2} *$ and SSFP cine imaging, breath-held inversion enhancement images were acquired following an intravenous administration of gadolinium contrast medium $(0.1$ and $0.15 \mathrm{mmol} / \mathrm{kg}$ at $3 \mathrm{~T}$ and $1.5 \mathrm{~T}$ respectively; Gadovist, Bayer $\mathrm{Plc}$, Germany).

Table 1 Participant characteristics

\begin{tabular}{lll}
\hline & $1.5 T$ & $3 T$ \\
\hline Number & 9 & 10 \\
Male;Female & $3: 6$ & $4: 6$ \\
Age (years) & $52[45.5-61.5]$ & $50[45.25-53]$ \\
Body-mass Index $\left(\mathrm{kg} / \mathrm{m}^{2}\right)$ & $22.9[20.1-26.9]$ & $25.9[22.5-29.4]$ \\
Ejection Fraction $(\%)$ & $63.6 \pm 4.9$ & $61.1 \pm 4.1$ \\
\hline
\end{tabular}

$\mathrm{N}(\%)$, mean $\pm \mathrm{SD}$, or median [interquartile range] 
Table 2 Normal values

\begin{tabular}{|c|c|c|c|c|c|c|}
\hline & $\begin{array}{l}\text { 1.5 T Pre-USPIO } \\
\text { R2* }\left(S^{-1}\right)\end{array}$ & $\begin{array}{l}1.5 \text { T Post-USPIO } \\
\mathrm{R}^{*}\left(\mathrm{~s}^{-1}\right)\end{array}$ & $\begin{array}{l}\text { 1.5 T Change } \\
\mathrm{R} 2^{*}\left(\mathrm{~s}^{-1}\right)\end{array}$ & $\begin{array}{l}3 \text { T Pre-USPIO } \\
\text { R2* }^{*}\left(S^{-1}\right)\end{array}$ & $\begin{array}{l}3 \text { T Post-USPIO } \\
\mathrm{R}^{*}\left(\mathrm{~s}^{-1}\right)\end{array}$ & $\begin{array}{l}\text { 1.5 T Change } \\
\mathrm{R} 2^{*}\left(\mathrm{~s}^{-1}\right)\end{array}$ \\
\hline Panmyocardial average & $33.5 \pm 5.4$ & $60.5 \pm 7.2$ & $26.5 \pm 7.3$ & $46.9 \pm 4.1$ & $84.2 \pm 12.4$ & $37.2 \pm 9.6$ \\
\hline Skeletal muscle & $34.7 \pm 4.2$ & $44.9 \pm 4.7$ & $10.2 \pm 5.8$ & $55.5 \pm 17.1$ & $59.8 \pm 6.6$ & $4.3 \pm 16.3$ \\
\hline Kidney & $16.6 \pm 2.0$ & $81.2 \pm 15.2$ & $64.6 \pm 16.1$ & $43.5 \pm 39.1$ & $115.2 \pm 28.1$ & $71.8 \pm 48.8$ \\
\hline Liver & $36.0 \pm 7.2$ & $252.3 \pm 34.3$ & $216.3 \pm 32.6$ & $65.3 \pm 21.2$ & $340.9 \pm 57.8$ & $275.6 \pm 69.9$ \\
\hline Spleen & $22.0 \pm 7.7$ & $358.3 \pm 59.5$ & $336.3 \pm 60.3$ & $51.2 \pm 21.1$ & $515.1 \pm 137.4$ & $463.9 \pm 136.7$ \\
\hline Blood & $11.3 \pm 4.1$ & $96.0 \pm 26.6$ & $84.7 \pm 27.2$ & $18.8 \pm 5.3$ & $91.5 \pm 20.9$ & $72.6 \pm 18.3$ \\
\hline Bone & $84.4 \pm 29.2$ & $154.3 \pm 62.0$ & $69.9 \pm 79.9$ & $330 \pm 168.7$ & $747.9 \pm 277.8$ & $417.9 \pm 370.3$ \\
\hline
\end{tabular}

Mean \pm SD

Optimal inversion time (TI) was determined on a slice-byslice basis using standard late-enhancement TI-scout protocols. The inversion-recovery late-enhancement short-axis slices were acquired using similar slice positions to the myocardial $\mathrm{T} 2 *$ imaging. The $\mathrm{T} 2 *$ acquisitions also included imaging of the liver, spleen and spine to allow quantification of USPIO accumulation within organs of the reticuloendothelial system.

\section{USPIO}

Intravenous infusion of USPIO (ferumoxytol, $4 \mathrm{mg} / \mathrm{kg}$; Rienso $^{\oplus}$, Takeda Italia, Italy) was performed immediately following the baseline magnetic resonance scan over at least $15-\mathrm{min}$ using a concentration of $2-8 \mathrm{mg} / \mathrm{mL}$, diluted in $0.9 \%$ saline or $5 \%$ dextrose. Hemodynamic monitoring was conducted throughout.

\section{Study protocol}

Volunteers received 2 MRI scans approximately $24 \mathrm{~h}$ apart (Fig. 1).

\section{Image analysis}

All T2*-weighted multi-gradient-echo images for each patient were analyzed using Circle CVI software (Circle CVI42, Canada). Regions of interest (ROI) were drawn in the heart using standard cardiac segmentation [24], and panmyocardial values averaged using segments $1-16$. Further ROI were drawn in skeletal muscle, kidney, liver, spleen, blood pool (from LV cavity) and bone marrow.

An experimentally determined threshold used in previous work [8] for the coefficient of determination $\left(\mathrm{r}^{2}>\right.$ 0.85) was used to exclude data that did not have an acceptable exponential decay when signal intensity (SI) was plotted against echo time. The inverse of the mean T2* $\left(\mathrm{R} 2{ }^{*}\right)$ for each ROI was then calculated to assess the uptake of USPIO, where the higher the value, the greater the USPIO accumulation.

Late gadolinium enhancement (LGE), ventricular volume and functional analyses were performed using Circle CVI software (Circle CVI42, Calgary, Canada). T2* data were collected immediately prior to USPIO administration. USPIO-enhanced T2* data were collected 24-25 h following ferumoxytol administration.

\section{Statistical analysis}

All statistical analysis was performed with GraphPad Prism, version 6 (GraphPad Software, San Diego, CA). To assess uptake of USPIO in tissues following single administration, $\mathrm{R} 2 *$ increase from pre to $24 \mathrm{~h}$ following USPIO were compared using repeated measures oneway ANOVA. Statistical significance was defined as twosided $p<0.05$.

\section{Results}

Twenty volunteer patients were recruited in total (10 at $1.5 \mathrm{~T}, 10$ at $3 \mathrm{~T})$. Forty MRI scans and 20 infusions of ferumoxytol were completed over the course of the study. Data from one participant at $1.5 \mathrm{~T}$ has been removed due to the presence of LGE, (which was included

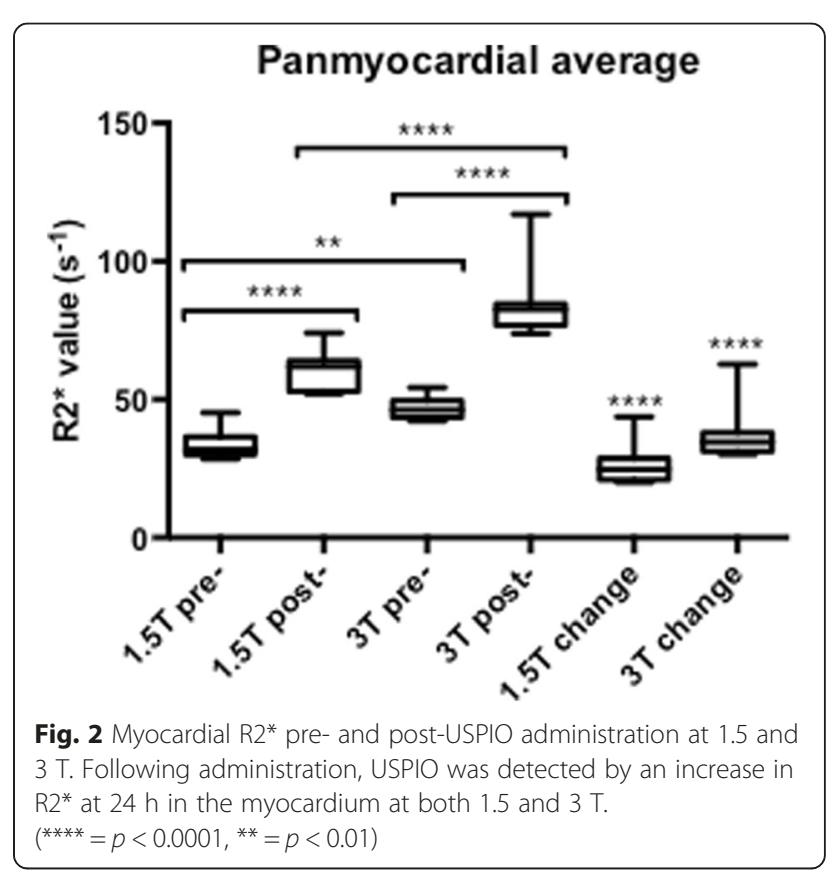


in the cardiac MR protocol so that we could exclude volunteers with any detectable cardiac MR abnormalities according to standard cardiac MR protocols). All other volunteers that were included had structurally normal hearts. One participant was prescribed antihypertensive medication but had a normal cardiac MR study and was normotensive so the data was retained for analysis. Administration of ferumoxytol was well tolerated with no adverse reactions reported during or immediately after administration in any of the participants.

Participants were predominantly middle aged, with greater numbers of women in both groups (Table 1).
There were no differences between $1.5 \mathrm{~T}$ and $3 \mathrm{~T}$ groups in BMI or ejection fraction at baseline.

A summary of results is shown in Table 2. At baseline, panmyocardial R2* values were greater at $3 \mathrm{~T}$ than $1.5 \mathrm{~T}$ $\left(46.9 \pm 4.1\right.$ versus $33.5 \pm 5.4 \mathrm{~s}^{-1}$, Fig. $\left.2, p<0.01\right)$ as expected. Baseline $\mathrm{R}^{*}$ values were also greater at $3 \mathrm{~T}$ in bone $(P<0.0001)$ but no baseline differences were seen between magnetic field strength in all other tissues (Fig. 3, $p>0.05$ for all). USPIO increased panmyocardial R2* values at $24 \mathrm{~h}$ in both $1.5 \mathrm{~T}$ and $3 \mathrm{~T}$ scanners $(p<0.0001$ for both). Post-USPIO panmyocardial R2* values were again greater at $3 \mathrm{~T}$ than $1.5 \mathrm{~T}$, as expected $(84.2 \pm 12.4$

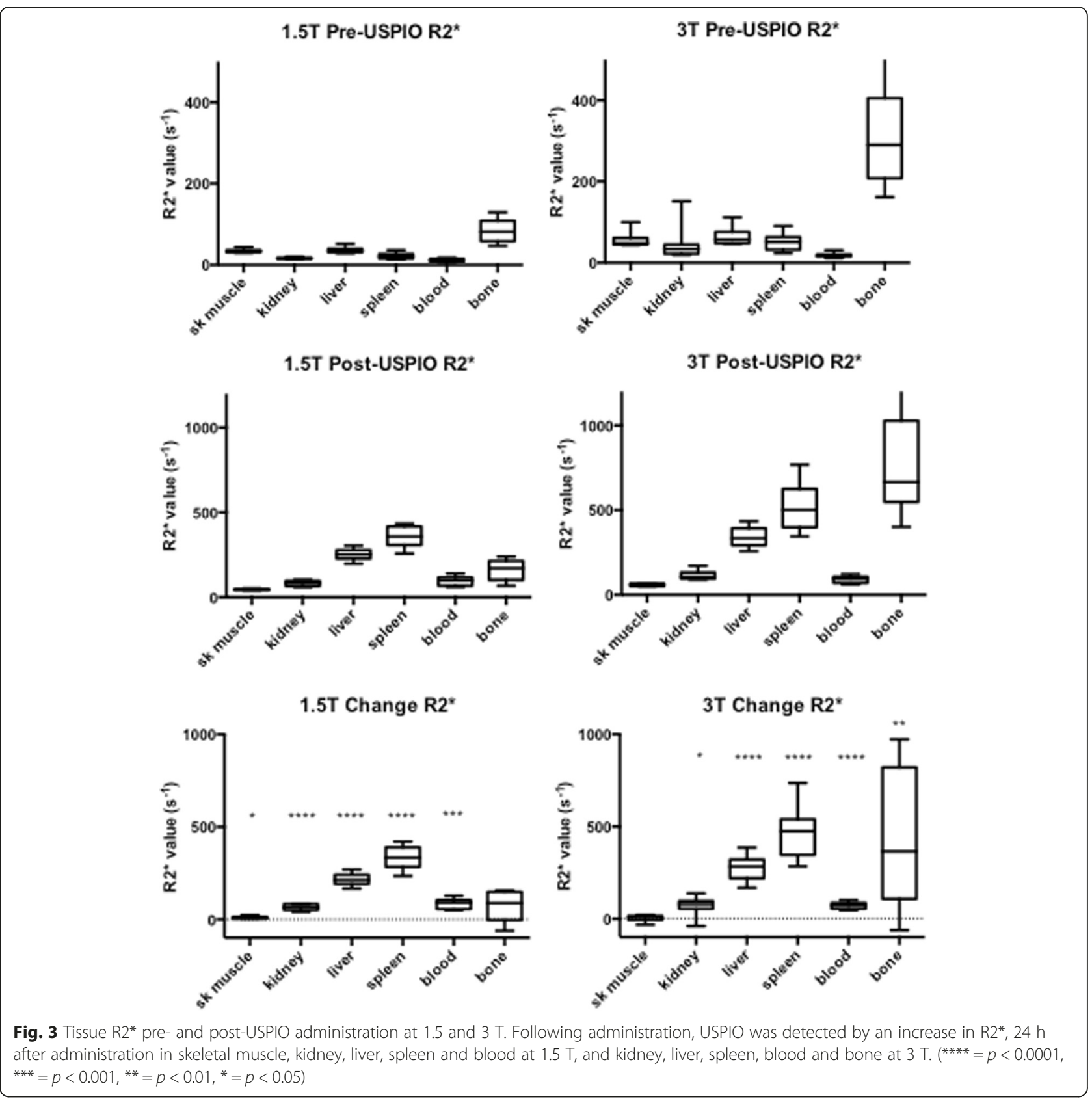


versus $\left.60.5 \pm 7.2 \mathrm{~s}^{-1}, p<0.0001\right)$. Panmyocardial change in R2* between baseline and $24 \mathrm{~h}$ post USPIO at $1.5 \mathrm{~T}$ was $26.5 \pm 7.3 \mathrm{~s}^{-1}$ and at $3 \mathrm{~T}$ was $37.2 \pm 9.6 \mathrm{~s}^{-1}(p<0.0001$ for both). Detectable increases in $\mathrm{R} 2 *$ were also observed at $24 \mathrm{~h}$ post-USPIO in skeletal muscle, kidney, liver, spleen and blood at $1.5 \mathrm{~T}$, and kidney, liver, spleen, blood and bone at 3 T. (Fig. 3, $p<0.05$ for all). BMI correlated with the panmyocardial R2 2 changes due to USPIO contrast (Fig. $4 ; r=0.72, p<0.001$ ).

\section{Discussion}

For the first time, we report a range of normal T2* values in the healthy human heart and other tissues $24 \mathrm{~h}$ after ferumoxytol administration at 1.5 and $3 \mathrm{~T}$. We also report problems, solutions and guidance in ferumoxytolenhanced $\mathrm{T}_{2} *$ image analysis.

Following administration, USPIO is detectable by $\mathrm{T} 2 *$ imaging in the myocardium and other tissues at both 1.5 and $3 \mathrm{~T}$. Tissues with small increases in R2* (less than the blood pool) are likely to represent detection of USPIOs within the intravascular space and include skeletal muscle (at $1.5 \mathrm{~T}$ only), myocardium and kidney. In contrast, R2* changes that are greater than the blood pool must be due to accumulation of USPIO, either through iron storage, uptake by macrophages or other phagocytes, or sequestered within tissue interstitium. In the absence of tissue biopsies, we cannot be certain, but as the most pronounced $\mathrm{R} 2 *$ changes were seen in the spleen, liver and bone marrow - organs of the reticuloendothelial system - it would appear likely that USPIO is incorporated quickly into tissue-resident phagocytes and macrophages.

Detection of USPIO enhancement in skeletal muscle at $1.5 \mathrm{~T}$ but not $3 \mathrm{~T}$ is due to generally noisier data seen across all tissues at $3 \mathrm{~T}$. Due to wider data confidence intervals, a larger sample size would be required to detected the same mean change in $\mathrm{R} 2 *$. The variation in data at $3 \mathrm{~T}$ is

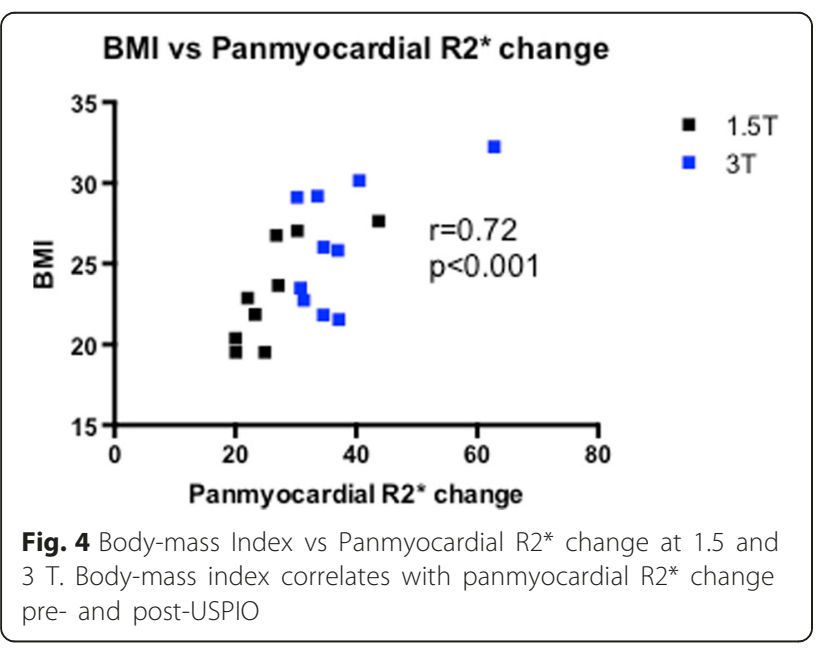

partly artifact in the images, but also because of the lower values at $3 \mathrm{~T}$ (USPIO has a faster $\mathrm{T}_{2} *$ decay time at $3 \mathrm{~T}$ ). With the same sampling echo times, there are fewer data points to construct the decay curve at $3 \mathrm{~T}$ than $1.5 \mathrm{~T}$ so our error in estimation also increases.

We chose $24 \mathrm{~h}$ post USPIO to re-image participants as myocardial signal attenuation at $24 \mathrm{~h}$ has shown to be optimal in the myocardium compared to later time points $[8,9]$. In view of this, scanning appointments were generally separated by $25 \mathrm{~h}$, and in practice, this regime worked well for both participants and MRI planning. According to previous work [8], we chose a weight-adjusted USPIO dose of $4 \mathrm{mg} \mathrm{Fe} / \mathrm{kg}$ body weight. However acknowledging that the distribution of USPIO following administration is predominantly in the organs of the reticuloendothelial system and blood pool, this may not be the optimum administration strategy as blood volume does not increase linearly with weight. We found a correlation between BMI and myocardial R2* change, probably due to increased blood pool USPIO concentration in those with higher BMI. We therefore suggest that a fixed dose approach may also be appropriate depending on the application.

Artifacts were commonly encountered with USPIOenhanced $\mathrm{T}^{*}$ imaging and made data analysis challenging. Post contrast artifacts at the blood-pool to myocardial interface were commonly seen and needed careful exclusion when selecting myocardial ROI. (Fig. 5A) This limited the assessment of USPIO accumulation at the endocardium. Similarly, blooming artifacts from nearby organs with high iron or blood pool USPIO content, such as lung and liver, commonly created signal deficits within the myocardium. In this situation, examination of $\mathrm{T} 2 *$ decay curves and excluding echo times influenced by artifact aided T2* decay curve fitting (Fig. 5).

The advantage of MRI mapping techniques is that visual assessment and objective quantification can be made using the same image, and these are now entering clinical practice. It seems likely that if UPSIO-enhanced MRI is adopted into clinical practice to detect tissue inflammation, $\mathrm{T} 2 *$ mapping would be used for image interpretation. However based on our experiences, we would recommend caution in interpreting maps alone. Signal attenuation seen on the T2* map may be interpreted as tissue USPIO accumulation, but may be due to blooming artifact from nearby susceptibility effects, and close examination of the $\mathrm{T} 2 *$ decay curve, and individual echoes if possible is suggested in order to distinguish accurately between tissue USPIO accumulation and artifact. In theory, setting an $r^{2}$ threshold as we did helps to exclude areas grossly affected by artifact. In practice however, regions with a seemingly acceptable $\mathrm{R}^{2}$ may still be influenced by artifact (Fig. 5). Manual exclusion of later echoes (influenced by artifact) from the curve may result in an improvement in $\mathrm{R}^{2}$ (a measure of how well the data 

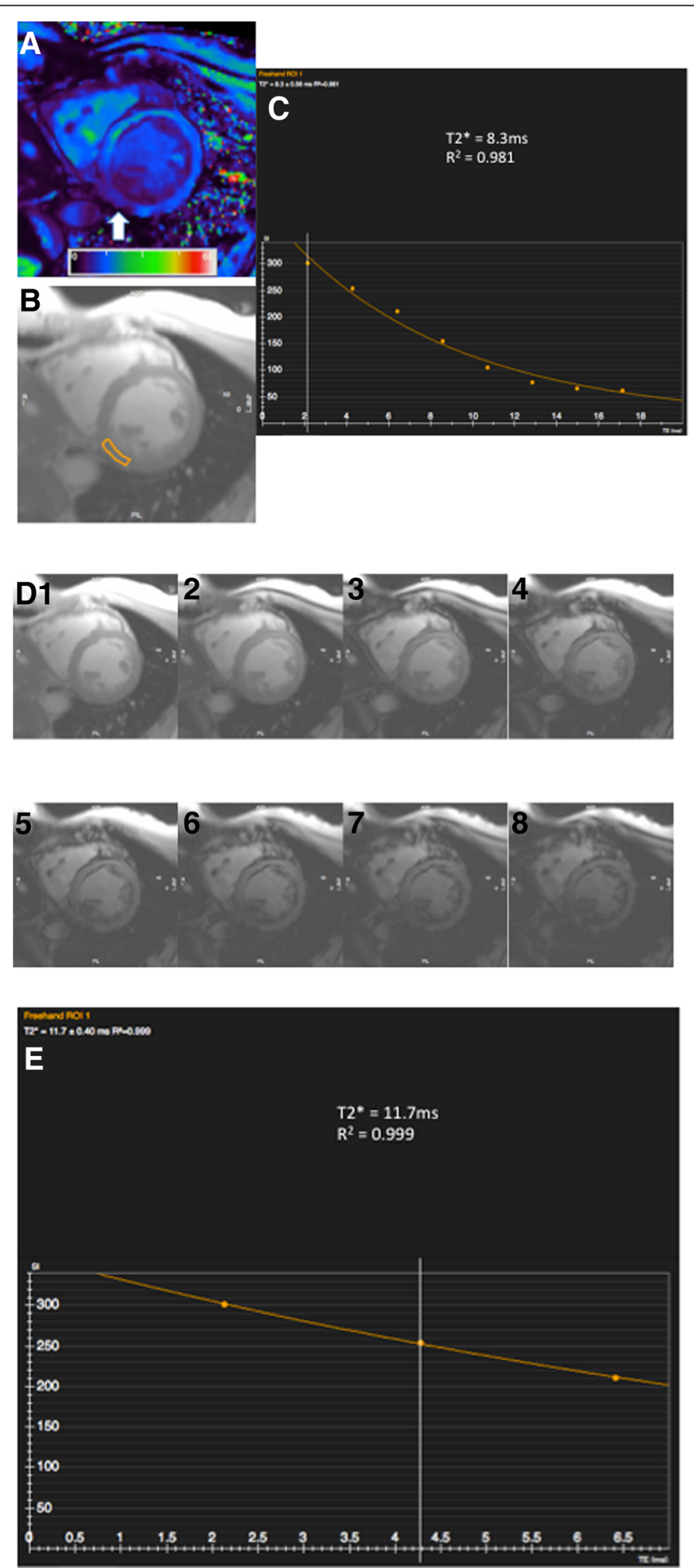

Fig. 5 (See legend on next page.) 
(See figure on previous page.)

Fig. 5 Inferior Blooming artifact. Example illustrating the challenge in assessing whether the inferior myocardial signal attenuation seen arrowed on the T2* colourmap (a, scale $0-60 \mathrm{~ms}$ ) is true or caused by artifact. Drawing a region of interest (b) and examining the decay curve (c) along with visualising individual echos (d $1-8)$ helps determine that this is a 'blooming artifact' from outside the heart is seen to influence echos 4-8. These can be manually removed, forming a new decay curve (e) with improvement in curve fitting ( $R^{2}$ value), although with fewer fitting points

points fit the curve), however there is the danger that reducing the number of fitting points will in fact reduce the overall sampling accuracy. Clearly, automated software capable of detecting and excluding artifact would be advantageous. This could be achieved by excluding, or applying less weight, to later echo times especially data points at a large distance from the initial decay curve trajectory $[25,26]$. It should be noted that like all other MRI sequences, poor data quality heavily influenced by breathing or movement artifact is generally non-interpretable and post processing using automated $\mathrm{T} 2 *$ decay curve fitting software is not likely to provide a remedy.

Echo times in this study were specific for cardiac imaging and were selected appropriately. Therefore they were not optimal for imaging tissues with $\mathrm{T} 2$ * values substantially higher or lower than myocardium. Native blood pool and post USPIO bone marrow (Fig. 6) provide examples of low and high $\mathrm{T} 2 *$ values respectively that we had difficulty accurately fitting a $\mathrm{T} 2$ * decay curve. With high T2* values, only a short part of the decay curve is plotted over the echo sampling time period, and often the signal has not decayed sufficiently for an accurate decay curve to be plotted. In contrast, regions with particularly short $\mathrm{T} 2$ * decay times have decayed to a level expected from background noise before sufficient data sampling has been made. Therefore fitting a decay curve from a small number (2-4) of echo times is clearly difficult, and often too much emphasis is
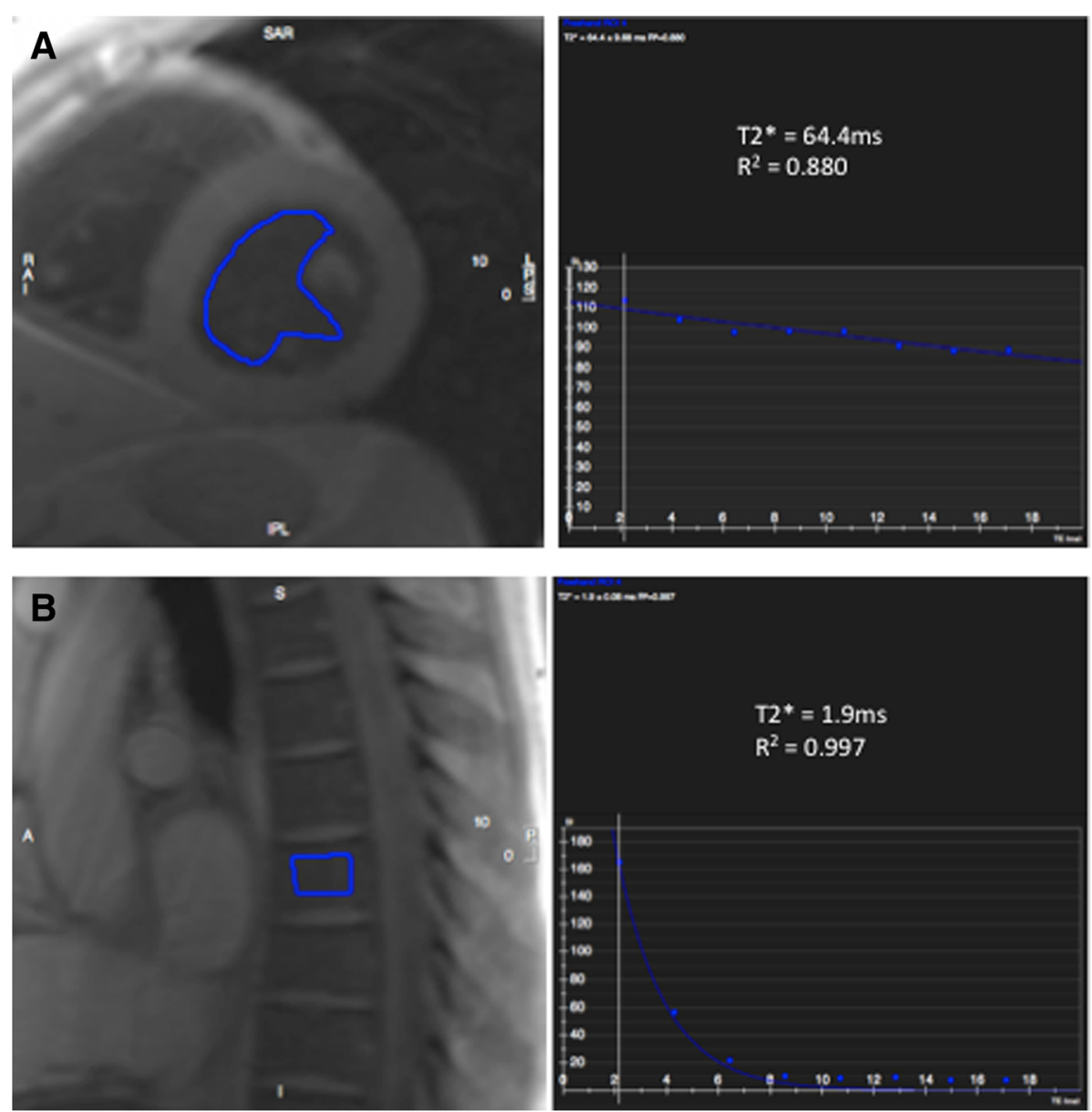

Fig. 6 Example of high and low T2* values. Regions of Interest with excessively low or high T2* value (pre-contrast blood pool, a, and post USPIO bone marrow, $\mathbf{b}$, respectively) can often be difficult to generate an accurate T2* decay curve. Imaging with tissue-spcific echo times will help generate more accurate $\mathrm{T}^{*}$ decay curves 
placed upon data decayed to the baseline level of background noise in order to generate a decay curve. Allowances can be made for background noise but are of limited value in this instance. We strongly advise applying tissue-specific echo times tailored to the expected $\mathrm{T} 2 \%$ value in order to achieve the most accurate decay curves possible.

\section{Limitations}

There are some limitations that should be taken into account when interpreting these data. First, this study has small numbers and a larger cohort of participants should be studied to further validate these normal values. Furthermore, due to geographical reasons, it was not feasible to scan the same participants at both centres so comparison cohorts at $1.5 \mathrm{~T}$ and $3 \mathrm{~T}$ were different. Despite this, both were healthy volunteers groups and displayed no differences at baseline so we do not feel this has impacted on the results. Finally, due to problems in interpreting high and low $\mathrm{T} 2 \%$ values as mentioned above, we recommend caution in interpreting some high non-cardiac R2* values; especially in the organs of the reticuloendothelial system at $3 \mathrm{~T}$. In these organs, the spread of $\mathrm{R}^{*}$ data above the median value appears wide. This is possibly caused by artifact and most evident at $3 \mathrm{~T}$, and may additionally explain why these regions have disproportionally high $\mathrm{R} 2 \%$ values.

\section{Conclusion}

We have shown that ferumoxytol-enhanced MRI is feasible at both $1.5 \mathrm{~T}$ and $3 \mathrm{~T}$, and suggest a range of expected normal values post-ferumoxytol in a range of tissues. Refinements of dose administration, optimization of acquired echo-times, careful image analysis, and development of post-processing and analysis software capable of excluding common artifacts, are essential to ensure reliable and robust quantification of tissue enhancement.

\section{Abbreviations}

LGE: late gadolinium enhancement; MRI: magnetic resonance imaging; ROI: regions of interest; USPIO: ultrasmall superparamagnetic particles of iron oxide.

\section{Funding}

This work was supported by the British Heart Foundation (FS/12/83). CS is supported by the Chief Scientist Office (ETM/266). SA and DEN are supported by the British Heart Foundation (FS/12/83; CH/09/002). DEN is the recipient of a Wellcome Trust Senior Investigator Award (WT103782AIA). Edinburgh Clinical Research Facility and the Clinical Research Imaging Centre are supported by NHS Research Scotland (NRS) through NHS Lothian. SS has received funding for this work via the British Heart Foundation Centre of Research Excellence award for the University of Edinburgh.

\section{Authors' contributions}

CS, SA, DN and SS designed the study, collected and analysed, data and drafted the manuscript. TM and CG analysed and interpreted data, and drafted the manuscript. MD, JP, SP, MP, RG, SM and PH designed the study and drafted the manuscript. All authors read and approved the manuscript.

\section{Competing interests}

The authors declare that they have no competing interests.

\section{Author details}

'British Heart Foundation/University Centre for Cardiovascular Science, University of Edinburgh, Edinburgh, UK. ${ }^{2}$ Clinical Research Imaging Centre, University of Edinburgh, Edinburgh, UK. ${ }^{3}$ Edinburgh Clinical Research Facility, University of Edinburgh, Edinburgh, UK. ${ }^{4}$ Department of Cardiology, Golden Jubilee National Hospital, Clydebank, UK. ${ }^{5}$ Department of Cardiology, Royal Brompton Hospital, London, UK.

Received: 19 January 2016 Accepted: 28 June 2016

Published online: 27 July 2016

\section{References}

1. Hahn PF, Stark DD, Lewis JM, Saini S, Elizondo G, Weissleder R, Fretz CJ, Ferrucci JT. First clinical trial of a new superparamagnetic iron oxide for use as an oral gastrointestinal contrast agent in MR imaging. Radiology. 1990; 175:695-700

2. Saini S, Stark DD, Wittenberg J, Brady TJ, Ferrucci JT. Ferrite particles: a superparamagnetic MR contrast agent for the reticuloendothelial system. Radiology. 1987;162:211-6.

3. Rogers JM, Lewis J, Josephson L. Visualization of superior mesenteric lymph nodes by the combined oral and intravenous administration of the ultrasmall superparamagnetic iron oxide, AMI-227. Magn Reson Imaging. 1994;12:1161-5.

4. Canet E, Revel D, Forrat R, Baldy-Porcher C, de Lorgeril M, Sebbag L, Vallee JP, Didier D, Amiel M. Superparamagnetic iron oxide particles and positive enhancement for myocardial perfusion studies assessed by subsecond T1weighted MRI. Magn Reson Imaging. 1993;11:1139-45.

5. Ros PR, Freeny PC, Harms SE, Seltzer SE, Davis PL, Chan TW, Stillman AE, Muroff LR, Runge VM, Nissenbaum MA. Hepatic MR imaging with ferumoxides: a multicenter clinical trial of the safety and efficacy in the detection of focal hepatic lesions. Radiology. 1995;196:481-8.

6. Kroft $L M$, Doornbos J, van der Geest RJ, van der Laarse A, van der Meulen H, de Roos A. Ultrasmall superparamagnetic particles of iron oxide (USPIO) MR imaging of infarcted myocardium in pigs. Magn Reson Imaging. 1998;16:755-63.

7. Taylor AM, Panting JR, Keegan J, Gatehouse PD, Amin D, Jhooti P, Yang GZ, McGill S, Burman ED, Francis JM, Firmin DN, Pennell DJ. Safety and preliminary findings with the intravascular contrast agent NC100150 injection for MR coronary angiography. J Magn Reson Imaging. 1999;9:220-7.

8. Alam SR, Shah ASV, Richards J, Lang NN, Barnes G, Joshi N, MacGillivray T, McKillop G, Mirsadraee S, Payne J, Fox KAA, Henriksen P, Newby DE, Semple SIK. Ultrasmall Superparamagnetic Particles of Iron Oxide in Patients With Acute Myocardial Infarction: Early Clinical Experience. Circ Cardiovasc Imaging. 2012;5:559-65.

9. Yilmaz A, Dengler MA, van der Kuip H, Yildiz H, Rosch S, Klumpp S, Klingel K, Kandolf R, Helluy X, Hiller KH, Jakob PM, Sechtem U. Imaging of myocardial infarction using ultrasmall superparamagnetic iron oxide nanoparticles: a human study using a multi-parametric cardiovascular magnetic resonance imaging approach. Eur Heart J. 2013;34:462-75.

10. Richards JMJ, Semple SI, MacGillivray TJ, Gray C, Langrish JP, Williams M, Dweck M, Wallace W, McKillop G, Chalmers RTA, Garden OJ, Newby DE. Abdominal Aortic Aneurysm Growth Predicted by Uptake of Ultrasmall Superparamagnetic Particles of Iron Oxide: A Pilot Study. Circ Cardiovasc Imaging. 2011;4:274-81.

11. McBride OMB, Berry C, Burns P, Chalmers RTA, Doyle B, Forsythe R, Garden OJ, Goodman K, Graham C, Hoskins P, Holdsworth R, MacGillivray TJ, McKillop G, Murray G, Oatey K, Robson JMJ, Roditi G, Semple S, Stuart W, van Beek EJR, Vesey A, Newby DE. MRI using ultrasmall superparamagnetic particles of iron oxide in patients under surveillance for abdominal aortic aneurysms to predict rupture or surgical repair: MRI for abdominal aortic aneurysms to predict rupture or surgery-the MA(3)RS study. Open Heart. 2015;2:e000190.

12. Trivedi RA. Identifying Inflamed Carotid Plaques Using In Vivo USPIOEnhanced MR Imaging to Label Plaque Macrophages. Arterioscler, Thromb, Vasc Biol. 2006;26:1601-6.

13. Trivedi RA, U-King-Im JM, Graves MJ, Cross JJ, Horsley J, Goddard MJ, Skepper JN, Quartey G, Warburton E, Joubert I, Wang L, Kirkpatrick PJ, Brown J, Gillard JH. In vivo detection of macrophages in human carotid atheroma: temporal dependence of ultrasmall superparamagnetic particles of iron oxide-enhanced MRI. Stroke. 2004;35:1631-5. 
14. Tang T, Howarth SPS, Miller SR, Trivedi R, Graves MJ, King-Im JU, Li ZY, Brown AP, Kirkpatrick PJ, Gaunt ME, Gillard JH. Assessment of inflammatory burden contralateral to the symptomatic carotid stenosis using highresolution ultrasmall, superparamagnetic iron oxide-enhanced MRI. Stroke. 2006:37:2266-70

15. Tang TY, Howarth SPS, Miller SR, Graves MJ, Patterson AJ, U-King-Im J-M, Li ZY, Walsh SR, Brown AP, Kirkpatrick PJ, Warburton EA, Hayes PD, Varty K, Boyle JR, Gaunt ME, Zalewski A, Gillard JH. The ATHEROMA (Atorvastatin Therapy: Effects on Reduction of Macrophage Activity) StudyEvaluation Using Ultrasmall Superparamagnetic Iron Oxide-Enhanced Magnetic Resonance Imaging in Carotid Disease. JAC. 2009;53:2039-50.

16. Anderson LJ, Holden S, Davis B, Prescott E, Charrier CC, Bunce NH, Firmin DN, Wonke B, Porter J, Walker JM, Pennell DJ. Cardiovascular T2-star (T2*) magnetic resonance for the early diagnosis of myocardial iron overload. Eur Heart J. 2001;22:2171-9.

17. Anderson LJ, Westwood MA, Holden S, Davis B, Prescott E, Wonke B, Porter $J B$, Walker JM, Pennell DJ. Myocardial iron clearance during reversal of siderotic cardiomyopathy with intravenous desferrioxamine: a prospective study using $\mathrm{T}^{*}$ cardiovascular magnetic resonance. Br J Haematol. 2004; 127:348-55.

18. Westwood MA, Anderson LJ, Firmin DN, Gatehouse PD, Lorenz CH, Wonke B, Pennell DJ. Interscanner reproducibility of cardiovascular magnetic resonance T2* measurements of tissue iron in thalassemia. J Magn Reson Imaging. 2003; 18:616-20.

19. Westwood M, Anderson $\sqcup$, Firmin DN, Gatehouse PD, Charrier CC, Wonke B, et al. A single breath-hold multiecho $\mathrm{T}^{*}$ cardiovascular magnetic resonance technique for diagnosis of myocardial iron overload. J Magn Reson Imaging. 2003;18:33-9.

20. Carpenter J-P, He T, Kirk P, Anderson LJ, Porter JB, Wood J, Galanello R, Forni G, Catani G, Fucharoen S, Fleming A, House M, Black G, Firmin DN, Pierre TGS, Pennell DJ. Calibration of myocardial iron concentration against T2-star Cardiovascular Magnetic Resonance. J Cardiovasc Magn Reson. 2009; $11: 1-2$

21. Kirk $P$, He T, Anderson $\sqcup$, Roughton M, Tanner MA, Lam WWM, Au WY, Chu WCW, Chan G, Galanello R, Matta G, Fogel M, Cohen AR, Tan RS, Chen K, Ng I, Lai A, Fucharoen S, Laothamata J, Chuncharunee S, Jongjirasiri S, Firmin DN, Smith GC, Pennell DJ. International reproducibility of single breathhold T2* MR for cardiac and liver iron assessment among five thalassemia centers. J Magn Reson Imaging. 2010;32:315-9.

22. Carpenter J-P, He T, Kirk P, Roughton M, Anderson LJ, de Noronha SV, Sheppard MN, Porter JB, Walker JM, Wood JC, Galanello R, Forni G, Catani G, Matta G, Fucharoen S, Fleming A, House MJ, Black G, Firmin DN, St Pierre TG, Pennell DJ. On T2* magnetic resonance and cardiac iron. Circulation. 2011;123:1519-28

23. Ruehm SG, Corot C, Vogt P, Kolb S, Debatin JF. Magnetic Resonance Imaging of Atherosclerotic Plaque With Ultrasmall Superparamagnetic Particles of Iron Oxide in Hyperlipidemic Rabbits. Circulation. 2001;103:415-22.

24. Cerqueira MD, Weissman NJ, Dilsizian V, Jacobs AK, Kaul S, Laskey WK, Pennell DJ, Rumberger JA, Ryan T, Verani MS, Myoca AHAWG . Standardized myocardial segmentation and nomenclature for tomographic imaging of the heart: A statement for healthcare professionals from the Cardiac Imaging Committee of the Council on Clinical Cardiology of the American Heart Association. J Am Soc Echocardiogr. 2002;15:463-7.

25. Shah $\mathrm{S}$, Xue H, Greiser A, Weale P, He T, Firmin DN, Pennell DJ, Zuehlsdorff S, Guehring J. Inline myocardial t2* mapping with iterative robust fitting. J Cardiovasc Magn Reson. 2011;13:P308.

26. He T, Gatehouse PD, Kirk P, Tanner MA, Smith GC, Keegan J, Mohiaddin RH, Pennell DJ, Firmin DN. Black-blood T2* technique for myocardial iron measurement in thalassemia. J Magn Reson Imaging. 2007:25:1205-9.

\section{Submit your next manuscript to BioMed Central and we will help you at every step:}

- We accept pre-submission inquiries

- Our selector tool helps you to find the most relevant journal

- We provide round the clock customer support

- Convenient online submission

- Thorough peer review

- Inclusion in PubMed and all major indexing services

- Maximum visibility for your research

Submit your manuscript at www.biomedcentral.com/submit
C Biomed Central 


\title{
(6) OPEN ACCESS
}

- Additional material is published online only. To view please visit the journal online (http://dx.doi.org/10.1136 heartjnl-2016-311018).

'British Heart Foundation/ University Centre for Cardiovascular Science, University of Edinburgh, Edinburgh, UK

${ }^{2}$ Clinical Research Imaging Centre, University of Edinburgh, Edinburgh, UK

${ }^{3}$ Clinical Research Facility, University of Edinburgh, Edinburgh, USA

${ }^{4}$ Department of Pathology, University of Edinburgh,

Edinburgh, UK

${ }^{5}$ Department of Cardiothoracic Surgery, Royal Infirmary of

Edinburgh, Edinburgh, UK

\section{Correspondence to}

Dr Colin G Stirrat, British Heart Foundation/University Centre for Cardiovascular Science, Room SU 305, Chancellor's Building, University of Edinburgh, 49 Little France Crescent, Edinburgh EH16 4SB, UK; colin. stirrat@ed.ac.uk

Received 12 December 2016 Revised 1 March 2017 Accepted 3 March 2017

Published Online First

22 June 2017

\section{CLlinked}

- http://dx.doi.org/10.1136 heartjnl-2017-311484

CrossMark

To cite: Stirrat CG, Alam SR, MacGillivray TJ, et al. Heart 2017:103:1528-1535.

\section{Ferumoxytol-enhanced magnetic resonance imaging assessing inflammation after myocardial infarction}

\author{
Colin G Stirrat, ${ }^{1}$ Shirjel R Alam, ${ }^{1}$ Thomas J MacGillivray, ${ }^{2,3}$ Calum D Gray, ${ }^{2,3}$ \\ Marc R Dweck, ${ }^{1}$ Jennifer Raftis, ${ }^{1}$ William SA Jenkins, ${ }^{1}$ William A Wallace, ${ }_{1}^{4}$ \\ Renzo Pessotto, ${ }^{5}$ Kelvin HH Lim, ${ }^{5}$ Saeed Mirsadraee, ${ }^{2}$ Peter A Henriksen, ${ }^{1}$ \\ Scott IK Semple, ${ }^{1,2}$ David E Newby ${ }^{1,2}$
}

\begin{abstract}
Objectives Macrophages play a central role in the cellular inflammatory response to myocardial infarction (MI) and predict subsequent clinical outcomes. We aimed to assess temporal changes in cellular inflammation and tissue oedema in patients with acute Ml using ultrasmallsuperparamagnetic particles of iron oxide (USPIO)-enhanced MRI.
\end{abstract}

Methods Thirty-one patients were recruited following acute $\mathrm{MI}$ and followed up for 3 months with repeated T2 and USPIO-enhanced T2* -mapping MRI. Regions of interest were categorised into infarct, peri-infarct and remote myocardial zones, and compared with control tissues.

Results Following a single dose, USPIO enhancement was detected in the myocardium until 24 hours $(p<0.0001)$. Histology confirmed colocalisation of iron and macrophages within the infarcted, but not the noninfarcted, myocardium. Following repeated doses, USPIO uptake in the infarct zone peaked at days $2-3$, and greater USPIO uptake was detected in the infarct zone compared with remote myocardium until days 10-16 $(p<0.05)$. In contrast, T2-defined myocardial oedema peaked at days 3-9 and remained increased in the infarct zone throughout the 3-month follow-up period $(p<0.01)$.

Conclusion Myocardial macrophage activity can be detected using USPIO-enhanced MRI in the first 2 weeks following acute MI. This observed pattern of cellular inflammation is distinct, and provides complementary information to the more prolonged myocardial oedema detectable using T2 mapping. This imaging technique holds promise as a non-invasive method of assessing and monitoring myocardial cellular inflammation with potential application to diagnosis, risk stratification and assessment of novel anti-inflammatory therapeutic interventions.

Trial registration number Trial registration number: 14663. Registered on UK Clinical Research Network (http://public.ukcrn.org.uk) and also ClinicalTrials.gov (https://clinicaltrials.gov/ct2/show/NCT02319278?term= DECIFER\&rank=2).

\section{INTRODUCTION}

More than one person every minute suffers a myocardial infarction (MI) in the USA. ${ }^{1}$ Despite improved interventional and medical treatments, mortality rates following MI remain high and many develop heart failure. ${ }^{1}$ Postinfarct inflammation plays a key role in the recovery of cardiac function, ${ }^{23}$ and it is a target for therapeutic manipulation to improve clinical outcomes.

After early neutrophil infiltration, monocytederived macrophages dominate the cellular infiltrate in the first 2 weeks following MI and sequentially coordinate digestion of damaged tissue and promotion of infarct healing. ${ }^{23}$ Optimal post-MI recovery is determined by the balance between proinflammatory and reparative macrophages, with uncontrolled early inflammation leading to adverse functional recovery. ${ }^{2-5}$

Ultrasmall superparamagnetic particles of iron oxide (USPIO) consist of an iron oxide core surrounded by a carbohydrate or polymer coating and are small enough to extravasate through diseased microvessels, where they are engulfed and concentrated by tissue-resident macrophages. ${ }^{6}$ Accumulation of USPIOs reduces T2* decay time and creates signal deficits that can be quantified and visualised using T2* MRI. ${ }^{7}{ }^{8}$ Thus, USPIOenhanced MRI can detect tissue-resident macrophage activity and identify cellular inflammation within tissues.

In current practice, T2-weighted MRI is used to evaluate myocardial oedema after MI. ${ }^{9}{ }^{10}$ However, these imaging techniques assess myocardial free water content and not active cellular inflammation. Development of a reliable non-invasive imaging technique capable of directly detecting myocardial cellular inflammation would be a major advance and could potentially facilitate risk stratification and therapeutic targeting of macrophages immediately after MI. Furthermore, this technique could provide diagnostic information, serial disease monitoring and a measure of treatment response in other conditions mediated by inflammatory cell infiltration of the heart.

In a recent proof-of-principle pilot study, ${ }^{7}$ we successfully detected inflammation following acute MI using USPIO-enhanced MRI. USPIO uptake, defined as the increase in R2* (1/T2*) 24 hours following administration, was seen in the infarct zone within the heart. In this study, we hypothesised that we could track the time course of cellular inflammation in the 3 -month period following acute MI using USPIO-enhanced MRI. Our primary aims were, first, to determine the duration and distribution of USPIO enhancement in tissues following a 
single infusion and, second, to assess the duration of macrophage infiltration of the heart by tracking myocardial USPIO enhancement using repeated USPIO administration, and compare this to measures of myocardial oedema using T2 mapping.

\section{METHODS}

This was an open-label observational cohort study. Patients were recruited within 7 days of acute MI. The study was performed in accordance with the Declaration of Helsinki, the approval of the local research ethics committee and the written informed consent of all participants.

\section{Subjects}

Participants were aged $18-80$ years and had sustained a recent MI according to the third universal definition of $\mathrm{MI},{ }^{11}$ with 12 -hour plasma troponin I concentration $\geq 5000 \mathrm{ng} / \mathrm{L}$. Exclusion criteria were known critical stenosis (>95\%) of left main stem, ongoing symptoms of angina, heart failure (Killip class $\geq 2$ ), renal failure (estimated glomerular filtration rate $<30 \mathrm{~mL} / \mathrm{min} / 1.73 \mathrm{~m}^{2}$ ) and contraindication to MRI or ferumoxytol infusion.

\section{Ultrasmall superparamagnetic particles of iron oxide}

Intravenous infusion of USPIO (ferumoxytol, Fe core $3.25 \mathrm{~nm}$, total particle diameter $17-31 \mathrm{~nm}, 4 \mathrm{mg} / \mathrm{kg}$; Rienso, Takeda Italia, Italy) was performed immediately following the baseline magnetic resonance scan over at least $15 \mathrm{~min}$ using a concentration of $2-8 \mathrm{mg} / \mathrm{mL}$, diluted in $0.9 \%$ saline or $5 \%$ dextrose. Haemodynamic monitoring was conducted throughout.

\section{Study protocol}

MRI and tissue histology were performed as described in the online supplementary material. A variable study protocol was used that was purposely designed for imaging at different time points after acute MI. Over a 3-month period following MI, patients received up to seven MRI scans and up to three infusions of USPIO. Two principal analyses were performed using data from the same participants.
First, to assess the duration of a single dose of USPIO, R2* values of only the participants who first received USPIO within 7 days of MI (21/30) were included in this analysis. They were followed on all subsequent MRI scans prior to receiving any further doses of USPIO (figure 1A). On receiving further USPIO, data were censored from the point of the second USPIO administration, hence the reduction in numbers during the course of the study.

Second, to assess the duration of myocardial macrophage activity, USPIO uptake was compared using repeated USPIO infusions over the 3-month follow-up period. All patients were included in this analysis. USPIO uptake was calculated by subtracting the baseline R2* value from values obtained at 24 hours following USPIO infusion (figure 1B). This allowed repeated assessment of myocardial USPIO uptake.

Following clinical guidance for the administration of ferumoxytol, participants were allowed a maximum of two doses of USPIO in a 1-month period, and a total of three over the 3 -month follow-up period to prevent iatrogenic iron overload. Therefore, USPIO-enhanced data were collected up to three times for each patient, and it was not possible to perform repeated USPIO-enhanced imaging at every time point in the same patient.

Finally, to assess myocardial oedema, T2 values were compared on unenhanced scans (prior to and at least 7 days following USPIO administration). All patients were included in this analysis.

\section{Image analysis}

All T2*-weighted multigradient-echo images for each patient were analysed using Circle CVI software (Circle CVI42, Canada). An experimentally determined threshold used in previous work ${ }^{7}$ for the coefficient of determination $\left(r^{2}>0.85\right)$ was used to exclude data that did not have an acceptable exponential decay when signal intensity was plotted against echo time. The relaxation rate, $\mathrm{R} 2 *$, is the inverse of the mean $\mathrm{T} 2 *$, and was calculated to assess the uptake of USPIO for each region

A

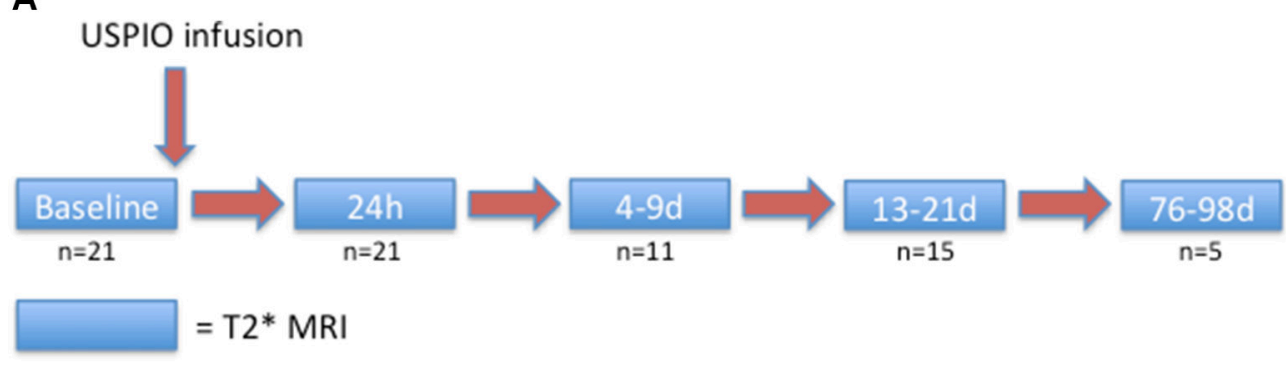

B

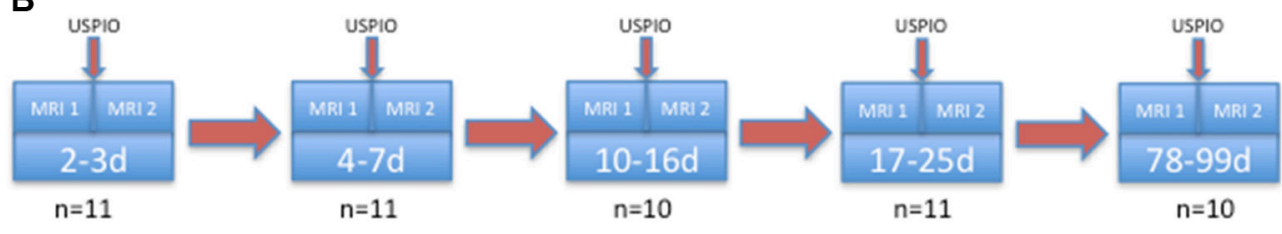

MRI $1=$ T2*, T2maps, wall motion, LGE

USPIO infusion immediately following MRI 1

MRI $2=$ T2* 24 h after USPIO

Figure 1 (A) Duration and distribution of USPIO enhancement after single-dose administration in the first week after MI. (B) Repeated myocardial uptake of USPIO following MI. MI, myocardial infarction; USPIO, ultrasmall superparamagnetic particles of iron oxide. 


\begin{tabular}{ll}
\hline Table 1 & Participant characteristics \\
\hline Number & 30 \\
\hline Age (years) & $58.4 \pm 9.8$ \\
\hline Sex & Male-29 (97) \\
& Female-1 (3) \\
Weight (kg) & $88.7 \pm 14.8$ \\
\hline Risk factors & \\
\hline Hypertension & $13(43)$ \\
\hline Diabetes mellitus & $8(27)$ \\
\hline Hypercholesterolaemia & $27(90)$ \\
\hline Family history & $14(47)$ \\
\hline Current or ex-smoker & $26(87)$ \\
\hline Infarct characteristics & \\
\hline STEMI & $25(83)$ \\
\hline Anterior & $10(33)$ \\
Inferior & $15(50)$ \\
\hline Lateral & $0(0)$ \\
\hline Diagnostic ECG to reperfusion (STEMI, & $72.5 \pm 16.8$ \\
min) & \\
\hline NSTEMI & $5(17)$ \\
\hline PCI & $26(87)$ \\
\hline Baseline ejection fraction (\%) & $48.5 \pm 11.2$ \\
\hline Infarct size (\% of LV mass) & $31.6 \pm 19.5$ \\
\hline Infarct volume (mL) & $43.9 \pm 27.5$ \\
\hline Plasma troponin (ng/L) & $31229 \pm 18563$ \\
\hline Subsequent CABG/biopsy) & $4 / 3$ \\
\hline Mea SD, N (\%) & \\
\hline
\end{tabular}

\section{Mean \pm SD, N (\%).}

CABG, coronary artery bypass grafting; LV, left ventricle; NSTEMI, non-ST-elevation myocardial infarction; PCl, percutaneous coronary intervention; STEMI, ST-elevation myocardial infarction.

of interest (ROI), where the higher the value, the greater the USPIO accumulation.

Late gadolinium enhancement (LGE), ventricular volume and functional analyses were determined manually using QMass software (Medis Medical Imaging Systems, Leiden, The Netherlands).

\section{ROI selection}

LGE images at the 3-month time point were used to determine the distribution and anatomy of the myocardial infarct, and served as reference images to which each series of T2 and T2* scans at each separate visit could be visually coaligned. This allowed myocardial ROIs to be selected on the reference short-axis LGE images and manually applied to T2* images corresponding to (1) infarct zone (defined by the short axis slice showing greatest LGE volume), (2) peri-infarct zone (half segment width immediately adjacent to LGE), and (3) remote myocardium (at least half segment width, opposite the infarct zone on the same short axis slice, and at least one full segment clear of any LGE). Other selected regions included (4) liver, (5) spleen, (6) bone marrow, (7) kidney, (8) blood pool, (9) aortic wall (inside arch) and (10) skeletal muscle.

T2 values were measured immediately prior to USPIO administration. USPIO-enhanced data were collected 24 hours following USPIO administration.

\section{Statistical analysis}

All statistical analyses were performed with GraphPad Prism, V.6 (GraphPad Software, San Diego, California, USA). To assess the duration of USPIO enhancement in tissues following single administration in the first week after MI, R2* values on subsequent MRI scans (prior to any further USPIO dosing) were compared using one-way analysis of variance (ANOVA) with Tukey's post hoc test for multiple comparisons.

To assess variation and duration of myocardial inflammation, USPIO uptake (R2* increase from pre to 24 hours following USPIO) was compared using one-way ANOVA with Tukey's post hoc test for multiple comparisons (repeated measures where appropriate). As a further method to assess USPIO accumulation within the heart, post-USPIO R2* 24 hours after administration (without baseline R2* subtraction) was compared in the same way.

Finally, to assess variation and duration of myocardial oedema, T2 values for infarct, peri-infarct and remote myocardium were compared again using one-way ANOVA with Tukey's post hoc test for multiple comparisons (repeated measures where appropriate). Statistical significance was defined as two sided, $\mathrm{p}<0.05$.

\section{RESULTS}

Thirty-one patients were recruited although 1 patient was excluded due to claustrophobia. A total of 147 MRI scans and 54 infusions of ferumoxytol were completed during the course of the study. Administration of ferumoxytol was well tolerated with no adverse reactions reported during or immediately after administration.

Participants were predominantly middle-aged men with current or previously diagnosed hypercholesterolaemia or smoking habit (table 1). Around half of the participants were hypertensive and had a family history of premature coronary heart disease. Twenty-five patients had ST-segment elevation MI, 5 patients had non-ST-segment elevation MI, 26 underwent percutaneous coronary intervention, and 4 had coronary artery bypass grafting $(\mathrm{CABG})$ surgery. The mean elevation in plasma troponin was $31200 \mathrm{ng} / \mathrm{L}$.

\section{Enhancement after single USPIO dose}

Twenty-one of the 30 participants received USPIO in the first week after MI and were included in this analysis.

USPIO was detected by an increase in R2* in all myocardial regions, blood pool, kidney and aortic wall until 24 hours, being cleared by 4-9 days (figure 2, online supplementary figure 1 and online supplementary table 1). USPIO was detected in bone marrow until 4-9 days and in liver and spleen until 13-21 days. There was no change in R2* following USPIO infusion in skeletal muscle.

Myocardial biopsies were taken from three of four patients undergoing CABG and revealed an abundance of inflammatory cells and early granulation tissue in keeping with a region of healing myocardium from around the infarct zone. Histological staining revealed colocalisation of the presence of iron (Prussian blue) and macrophages (CD68). No staining for iron or macrophages was seen within the adjacent regions of healthy myocardium (figure 2).

\section{Time course of enhancement with repeated USPIO administration}

There were no differences in baseline (pre-USPIO) R2* value between infarct and remote myocardium at all time points. Time course variation in USPIO uptake was seen in the infarct zone over the 3-month period, peaking at days 2 and 3 (figures 3a, 4 and online supplementary table $2 \mathrm{a}$ ). There was no time course variation in USPIO uptake in the peri-infarct and remote myocardium or extra-cardiac tissues over the 3-month follow-up period. 

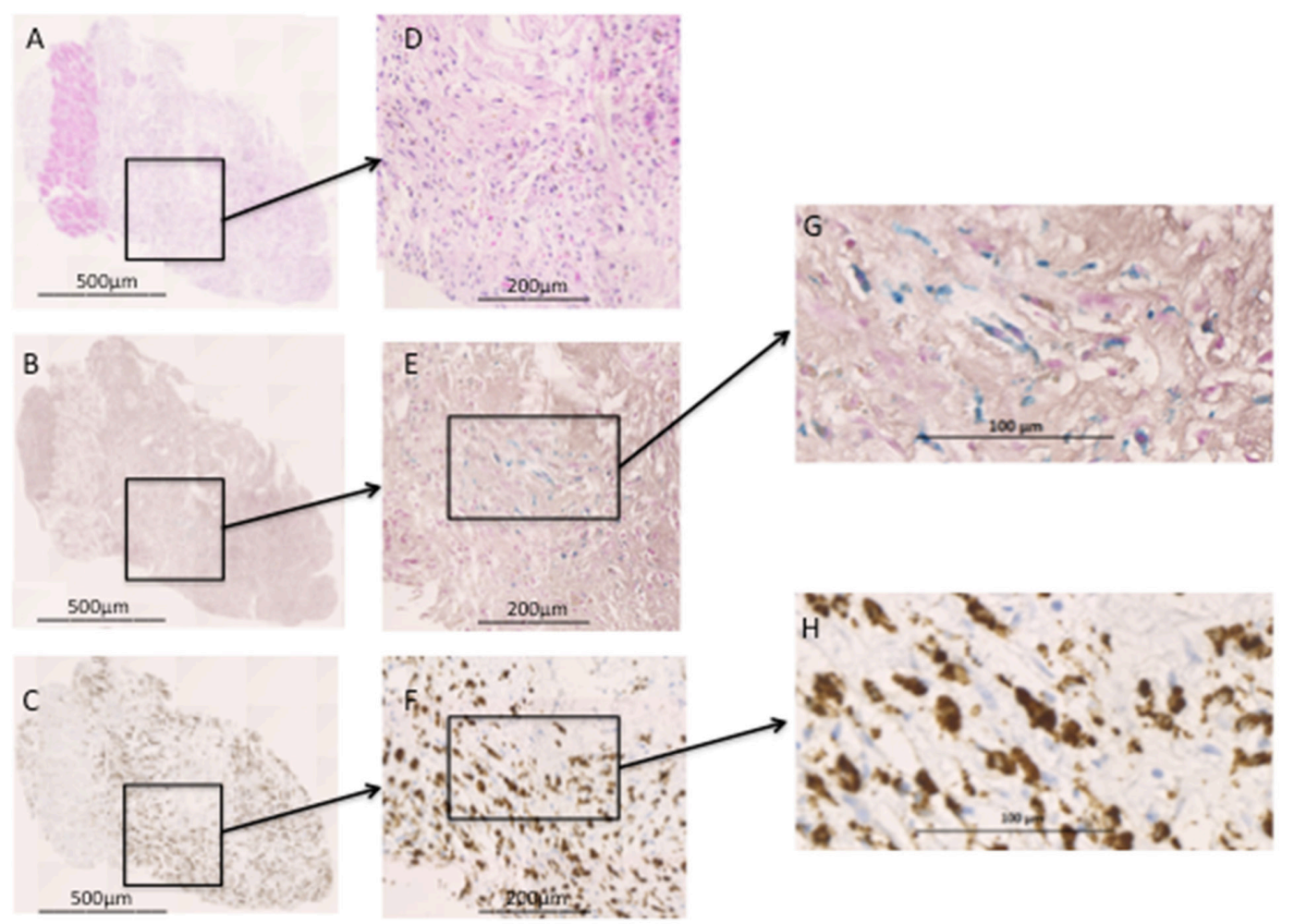

Figure 2 Histology section of trucut biopsy from infarcted myocardium. (A) Haematoxylin and eosin ( $\times 5$ magnification) stain displaying a thin region of viable healthy myocardium (strip of dark pink) surrounded by infarcted myocardium (lighter pink). (D) Infarcted myocardium shows an abundance of inflammatory cells and early granulation formation. (B) Prussian blue $(\times 5)$ staining revealing intracellular iron $\left(E_{,} \times 20\right.$ and $\left.G, \times 50\right)$, not seen in the region of healthy myocardium. (C) CD68 ( $\times 5)$ staining revealing macrophages within the infarcted myocardium $(F, \times 20$ and $H, \times 50)$, again not seen in healthy myocardium. $\mathrm{G}+\mathrm{H}$ show colocalisation of iron within macrophages.

Compared with remote myocardium, greater USPIO uptake was seen in the infarct zone until days $10-16\left(\right.$ mean $+56 \mathrm{~s}^{-1}$; 95\% CI 33 to $79 ; \mathrm{p}<0.001,+47 \mathrm{~s}^{-1} ; 95 \%$ CI 31 to $62 ; \mathrm{p}<0.0001$, and $+33 \mathrm{~s}^{-1} ; 95 \% \mathrm{CI} 4$ to $61 ; \mathrm{p}<0.05$ at $2-3$ days, $4-7$ days and 10-16 days, respectively) but not thereafter (figure 3a). Similarly, comparing R2* 24 hours following USPIO (without baseline $\mathrm{R} 2$ * subtraction), greater USPIO uptake was again evident in infarct myocardium compared with remote myocardium until 10-16 days (mean $+60 \mathrm{~s}^{-1} ; 95 \%$ CI 33 to $86 ; \mathrm{p}<0.01,+49 \mathrm{~s}^{-1}$;
95\% CI 21 to $77 ; \mathrm{p}<0.01$, and $+45 \mathrm{~s}^{-1} ; 95 \%$ CI 2 to $87 ; \mathrm{p}<0.05$ at 2-3 days, $4-7$ days and 10-16 days, respectively, figure $3 \mathrm{~b}$ and online supplementary table $2 \mathrm{~b}$ ).

\section{Assessment of myocardial oedema using T2 mapping}

Following acute MI, time course variation in infarct and periinfarct T2 values was seen (see figure 5 and online supplementary table 3). No time course variation in $\mathrm{T} 2$ value was seen

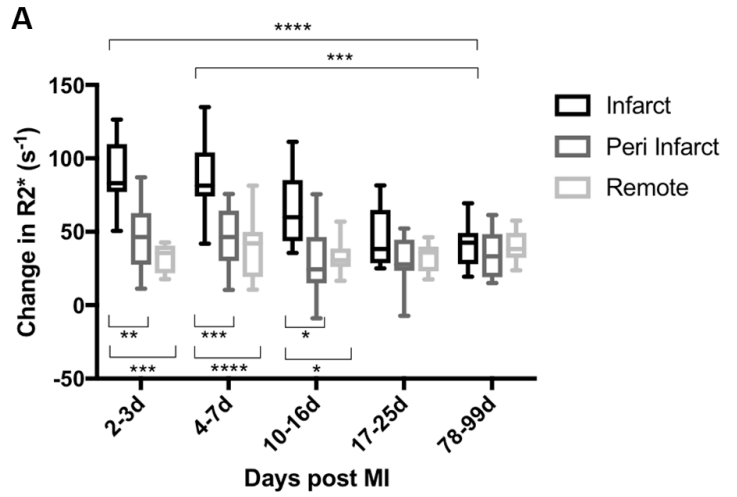

B

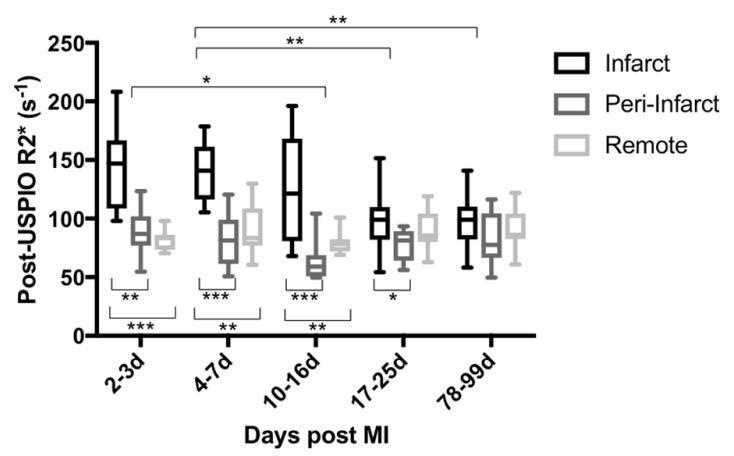

Figure 3 (A) USPIO uptake in myocardium after MI with repeated USPIO administration. (B) R2* 24 hours following USPIO (without subtraction of baseline values). Time course variation in USPIO uptake was seen in the infarct zone peaking at days 2 and 3. No variation of USPIO uptake was seen in peri-infarct and remote myocardium. Compared with remote myocardium, increased USPIO uptake was seen in the infarct zone until days 10-16 post MI. $\left({ }^{* * *} p<0.0001,{ }^{* *} p<0.001,{ }^{* *} p<0.01,{ }^{*} p<0.05\right)$. Compared with remote myocardium, greater post-USPIO R2 ${ }^{*}$ was seen in the infarct zone until days $10-16$ post MI. $\left({ }^{* *} p<0.001,{ }^{* *} p<0.01,{ }^{*} p<0.05\right)$. A+B: $n=11$ (days $2-3,4-7$ and $\left.17-25\right), 10$ (days 10-16) and 10 (days 78-99). $\mathrm{MI}$, myocardial infarction; USPIO, ultrasmall superparamagnetic particles of iron oxide. 
LGE
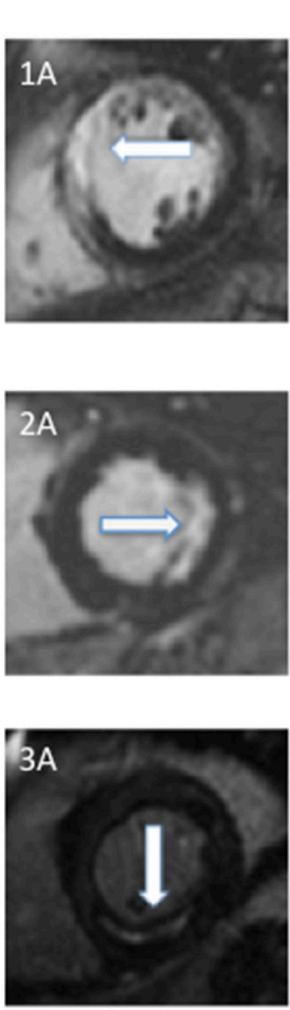

R2* Map
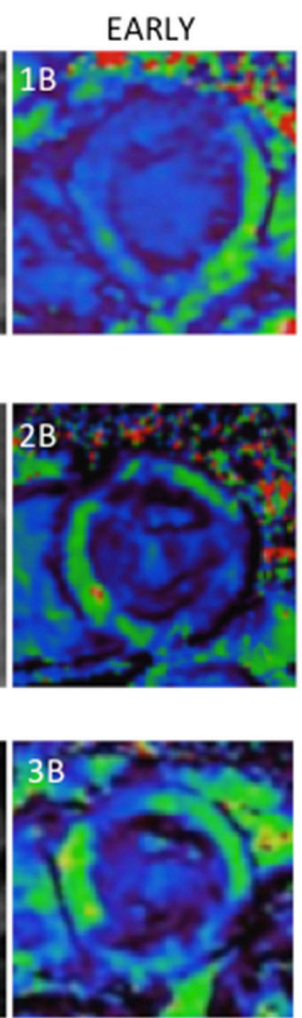

3MONTHS
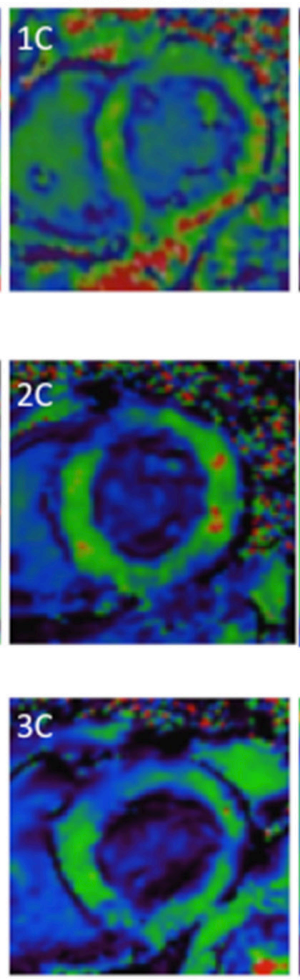

T2 MAP

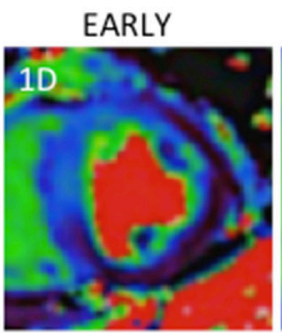

\section{MONTHS}

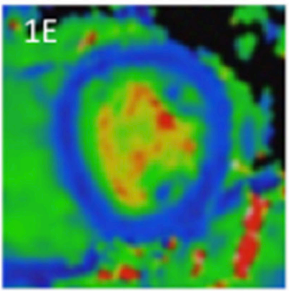

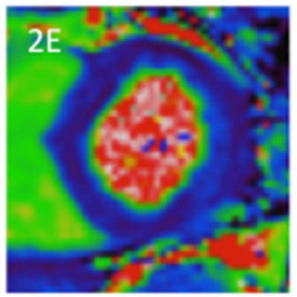
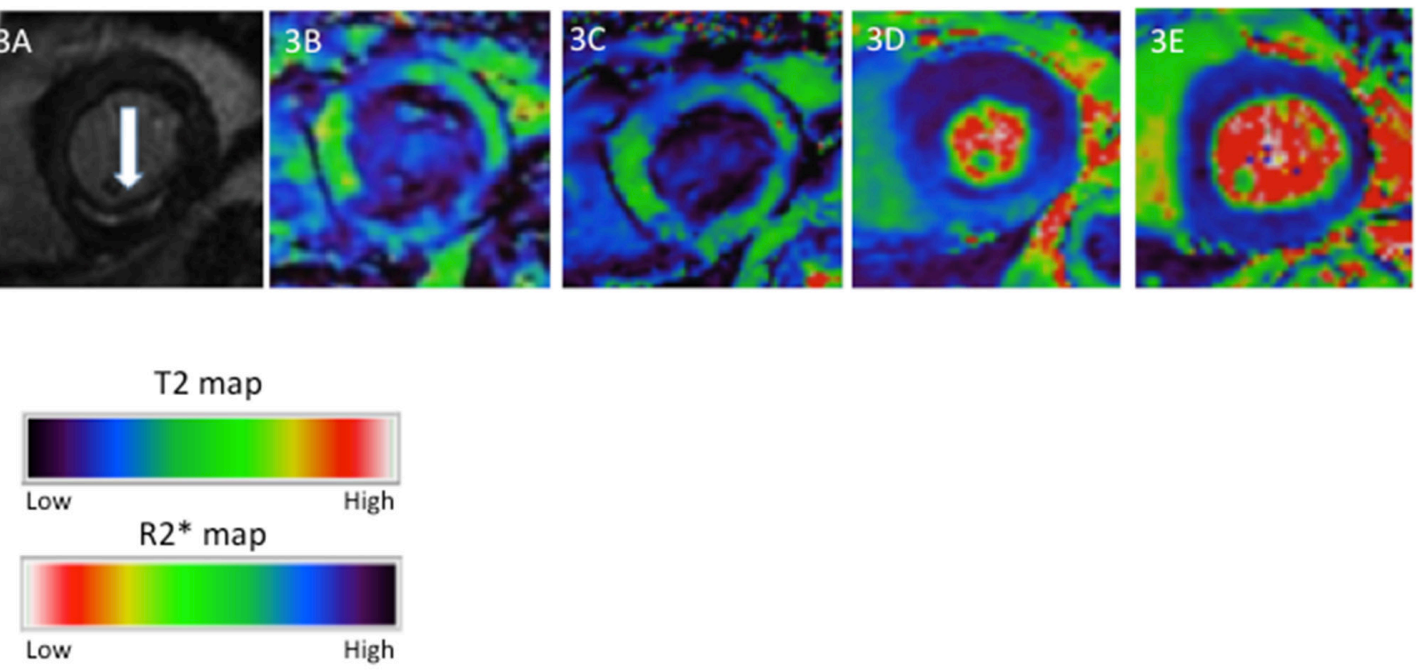

Figure 4 Examples of myocardial oedema and USPIO enhancement in the infarct zone after MI. Three examples of MI (1—anteroseptal, 2-lateral and 3-inferior) illustrating LGE, USPIO enhancement (R2* map) and oedema (T2 map) at early (up to 10 days) and late (3 months) time points. Early inflammation and oedema seen on R2* (dark region) and T2 maps (light region), respectively, have improved or resolved by 3 months. LGE, late gadolinium enhancement; MI, myocardial infarction; USPIO, ultrasmall superparamagnetic particles of iron oxide.

in remote myocardium. Compared with remote myocardium, greater myocardial T2 value was detected in the infarct zone and remained present throughout the 3 -month follow-up period (mean +12 ms, 95\% CI 7 to $18, \mathrm{p}<0.001 ;+14 \mathrm{~ms}, 95 \%$ CI 9 to $18, \mathrm{p}<0.0001$; $+15 \mathrm{~ms}, 95 \%$ CI 6 to $24, \mathrm{p}<0.01$; $+12 \mathrm{~ms}$, $95 \%$ CI 5 to $19, \mathrm{p}<0.01$ and $+6 \mathrm{~ms}, 95 \%$ CI 2 to $9, \mathrm{p}<0.01$ at days $1-2,3-9,10-16,17-24$ and 77-98, respectively).

\section{DISCUSSION}

For the first time, we have simultaneously investigated cellular inflammation and tissue oedema for 3 months following acute MI using USPIO-enhanced T2* and T2-mapping MRI. We have demonstrated that while tissue oedema persists for at least 3 months, cellular inflammation in the infarct zone is transient and lasts for only 2 weeks following MI. The time course of USPIO uptake following MI demonstrates a pattern of macrophage inflammation that is distinct to tissue oedema caused by the loss of capillary integrity. This suggests that while capillary integrity may take several months to resolve, cellular inflammation and specifically macrophage activity predominates in the first 2 weeks following MI.

USPIO can be used as an MRI contrast agent as it is immediately apparent in the blood pool following intravenous administration. ${ }^{12}$ Tissue enhancement with USPIO following inflammation has been suggested to occur as a result of either partitioning of USPIOs into the tissues because of the loss of capillary integrity alone or as a result of macrophage phagocytic activity clearing tissue-resident particles. ${ }^{8}$ We here provide confirmatory evidence that USPIO enhancement predominantly occurs as a result of cellular inflammation within the myocardium. We used T2 mapping to describe the loss of capillary integrity following MI. Using this comparator, we demonstrate that there is a discontinuity between the prolonged (up to 3 months) T2-defined time course of tissue oedema, and the brief (first 2 weeks) T2*-defined time-limited cellular inflammation. This observed USPIO uptake in the infarct zone for up to 2 weeks following MI is consistent with data from previous studies demonstrating that tissue-resident macrophages are active and 


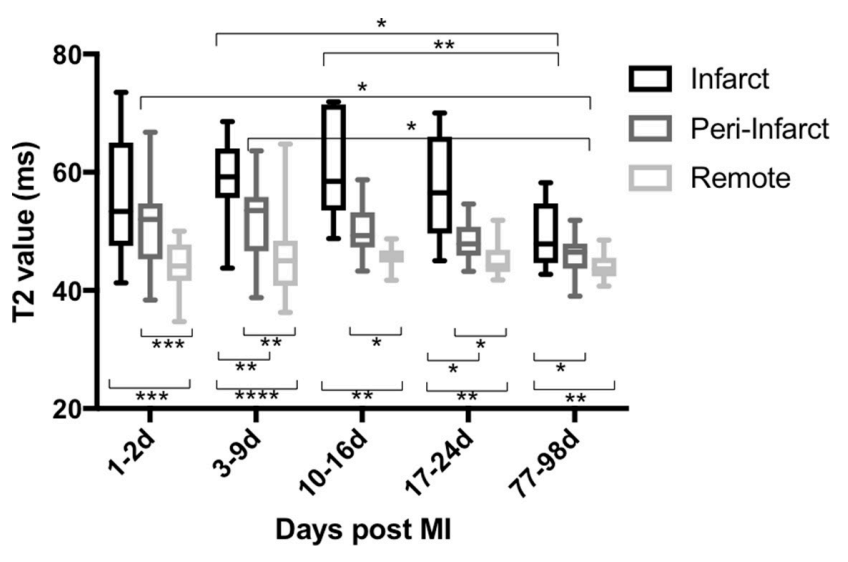

Figure 5 T2 value in the myocardium after MI. Time course variation in infarct and peri-infarct T2 was seen in the 3-month period following $\mathrm{Ml}$, both peaking at days 3-9. Compared with remote myocardium, higher $\mathrm{T} 2$ was seen in the infarct zone throughout the duration of the study and in the peri-infarct zone until days $17-24\left({ }^{* * * *} p<0.0001\right.$, $\left.{ }^{* * *} p<0.001,{ }^{* *} p<0.01,{ }^{*} p<0.05\right) ; n=15$ (days $1-2$ ), 14 (days $3-9$ and 77-98), 8 (days 10-16) and 10 (days 17-24). Ml, myocardial infarction.

predominate within the infarcted myocardium in the first 2 weeks after MI. $^{35}$ In addition, we obtained myocardial biopsies in patients undergoing CABG surgery within 2 weeks of MI and 24 hours after USPIO administration. We demonstrated marked colocalisation of iron staining with tissue-resident macrophages in infarcted but not in non-infarcted tissue. It is not possible to resolve specific USPIOs on histology, and we cannot discount the possibility that there is some contribution from postinfarct haemosiderin staining. However, we would contend that the majority of iron staining is attributable to USPIO uptake given the selective, transient and marked R2* enhancement on MRI, and the relatively constant contribution from haemosiderin during the period of assessment. Moreover, we did not detect substantial quantities of iron that were free in the extracellular space and were not associated with areas of macrophage infiltration.

Following a single infusion in the first week after MI, USPIO enhancement is seen in all regions of the myocardium, but greatest within the infarct zone, after 24 hours. USPIO enhancement is also observed until 4-9 days in bone marrow and until 13-21 days in the liver and spleen. Therefore, the duration of USPIO enhancement is longest in the mononuclear phagocyte-rich reticuloendothelial system. It is likely that resident phagocytes within these organs ingest USPIOs from circulating blood. Efflux of myocardial macrophages containing USPIO may also contribute to the reticuloendothelial system $\mathrm{T} 2 *$ signal. Ultimately, once within macrophages, the iron oxide core is broken down by lysosomes, providing a supply of iron ions for haemoglobin synthesis. ${ }^{13} 14$

The observed duration of enhancement following a single infusion of USPIO in tissues helped guide the minimum time interval between repeated USPIO administrations. Complete washout of the previous dose of USPIO, allowing baseline R2* to return to normal, is required to reassess uptake of USPIO accurately, without influence from previous USPIO administration. We have shown that USPIO is no longer detectable in the myocardium at 1 week following intravenous administration, and we suggest that 1 week is the minimum interval between repeated doses of USPIO.

USPIO is detectable in the peri-infarct and remote myocardium, kidney and aortic wall at 24 hours following administration. However, the amplitude of R2* change is less than that of blood pool, suggesting this is likely to be due to a diluted effect of blood-pool USPIO and not macrophage uptake of USPIO
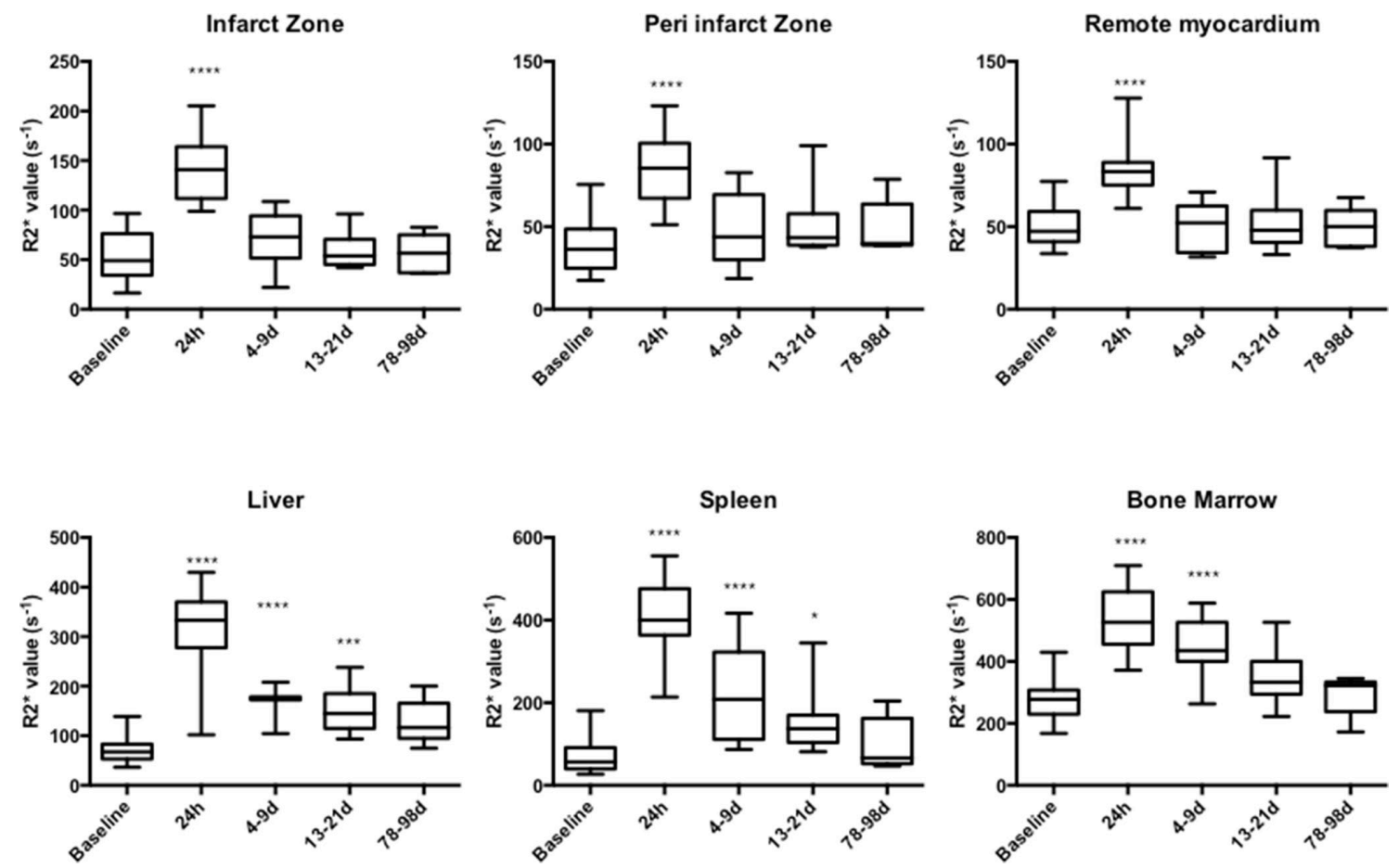

Figure 6 USPIO enhancement after single-dose administration in the first week following MI. Following single-dose administration, USPIO enhancement was detected in all myocardial regions at 24 hours. USPIO was detected in bone marrow until 4-9 days, and spleen and liver until 13-21 days $\left({ }^{* * * *} p<0.0001,{ }^{* * *} p<0.001,{ }^{*} p<0.05\right) ; n=21$ (baseline, 24 hours), 11 (days 4-9), 15 (days 13-21) and 5 (days 78-99). MI, myocardial infarction; USPIO, ultrasmall superparamagnetic particles of iron oxide. 
within these tissues. Although there appears a trend to suggest that the peri-infarct zone displays an early relative increase in USPIO uptake compared with remote myocardium, this failed to reach statistical significance.

Previous studies have suggested that the remote myocardium may exhibit USPIO and macrophage accumulation. ${ }^{7} 815$ We have shown here that there is no time course variation in USPIO uptake in remote myocardium after MI, and the small $\mathrm{R} 2$ * increase following USPIO is likely to be reflective of intravascular capillary-bed blood-pool USPIO rather than more widespread macrophage activity throughout the myocardium. This is consistent with our recent report of similar R2* increases in the myocardium of healthy volunteers which we again attribute to the blood pool signal of USPIO. ${ }^{16}$

Although no patients in this study displayed imaging evidence of myocardial haemorrhage, we were able to account for the presence of microscopic iron due to haemorrhage by subtracting baseline R2*. Therefore, any R2* increase detected is due to accumulation of USPIO and not iron from myocardial haemorrhage. We acknowledge that intramyocardial haemorrhage occurring between the pre and 24 hours post USPIO scans could theoretically increase R2*, but the earliest we imaged patients was at least 24 hours after MI, by which time haemorrhage is likely to have taken place.

We chose T2 mapping to assess myocardial oedema as it provides improved sensitivity and addresses some of the problems with subjective image interpretation using traditional T2-weighted techniques. ${ }^{17} 18$ The finding of ongoing myocardial oedema in the infarct zone at 3 months after MI may at first appear surprising. However, several groups have described the long-lasting presence of myocardial oedema, often up to 6 months after MI. ${ }^{19} 20$ This suggests that it may take many months for complete resolution of injury and return of normal vascular homeostasis and function.

What are the clinical applications of our technique? We have here used the 'model' of acute MI and demonstrated the predicted time course of tissue-resident macrophage uptake using USPIOs. We therefore suggest that this technique has utility in defining the extent of myocardial cellular inflammation that has potential utility in risk stratification. Perhaps more importantly, USPIO-enhanced MRI may prove a useful tool to assess the response to novel anti-inflammatory interventions targeted at reducing myocardial cellular inflammation to improve clinical outcomes. Such a study was carried out at our centre and has recently been published. ${ }^{21}$ Currently, there is no USPIO licensed as a contrast agent for routine clinical MRI, which limits its widespread use. However, the most exciting application would be to extend this technique to help diagnose, monitor disease progression and assess therapeutic intervention in myocardial diseases associated with and mediated by macrophage infiltration. Such diseases include myocarditis, cardiac transplant rejection and cardiac sarcoidosis, and a multicentre study is currently under way to assess this (EUDRACT 2013-002336-24). This would be important given the lack of simple definitive diagnostic tests in these conditions, the challenges of unreliable cardiac biopsies, and the need for rapid assessments of disease progression and therapeutic interventions.

In conclusion, we have successfully imaged, quantified and validated macrophage inflammation and myocardial oedema over 3 months in patients following MI using USPIO-enhanced T2* MRI and T2 mapping. This technique can provide a non-invasive method for the diagnosis and monitoring of tissue inflammatory macrophage activity in the heart. It also provides a potential platform on which to assess existing and novel therapeutic interventions that might modify the inflammatory process after MI and in other inflammatory processes affecting the heart.

\section{Key messages}

What is already known on this subject?

- Ultrasmall superparamagnetic particles of iron oxide (USPIO) are ingested by tissue macrophages that can be visualised using MRI to highlight areas of inflammation after myocardial infarction (MI).

What might this study add?

- The pattern of cellular inflammation after MI is distinct to tissue oedema. Myocardial macrophage activity is evident for 2 weeks after MI compared with the more prolonged period of myocardial oedema.

How might this impact on clinical practice?

- USPIO-enhanced MRI may be of value in diagnosing active $\mathrm{MI}$ and ultimately may provide an additional diagnostic tool or provide risk stratification and serial imaging in assessing inflammatory processes affecting the heart.

Funding This work was supported by the British Heart Foundation (FS/12/83). CGS is supported by the Chief Scientist Office (ETM/266). SA and DEN are supported by the British Heart Foundation (FS/12/83; CH/09/002). DEN is the recipient of a Wellcome Trust Senior Investigator Award (WT103782AIA). Wellcome Trust Clinical Research Facility and the Clinical Researchlmaging Centre are supported by NHS Research Scotland (NRS) through NHS Lothian.

Competing interests None declared.

Provenance and peer review Not commissioned; externally peer reviewed.

Open Access This is an Open Access article distributed in accordance with the terms of the Creative Commons Attribution (CC BY 4.0) license, which permits others to distribute, remix, adapt and build upon this work, for commercial use, provided the original work is properly cited. See: http://creativecommons.org/ licenses/by/4.0/

(c) Article author(s) (or their employer(s) unless otherwise stated in the text of the article) 2017. All rights reserved. No commercial use is permitted unless otherwise expressly granted.

\section{REFERENCES}

1 As G, Mozaffarian D, Roger VL, et al. Heart disease and stroke statistics-2014 update: a report from the - PubMed - NCBI. Circulation 2014;129:e28-92.

2 Nahrendorf M, Swirski FK, Aikawa E, et al. The healing myocardium sequentially mobilizes two monocyte subsets with divergent and complementary functions. J Exp Med 2007;204:3037-47.

3 Nahrendorf M, Pittet MJ, Swirski FK. Monocytes: protagonists of infarct inflammation and repair after myocardial infarction. Circulation 2010;121:2437-45.

4 Panizzi P, Swirski FK, Figueiredo JL, et al. Impaired infarct healing in atherosclerotic mice with Ly-6C(hi) monocytosis. J Am Coll Cardiol 2010;55:1629-38.

5 Tsujioka $\mathrm{H}$, Imanishi T, Ikejima $\mathrm{H}$, et al. Impact of heterogeneity of human peripheral blood monocyte subsets on myocardial salvage in patients with primary acute myocardial infarction. J Am Coll Cardiol 2009;54:130-8.

6 Ruehm SG, Corot C, Vogt P, et al. Magnetic resonance imaging of atherosclerotic plaque with ultrasmall superparamagnetic particles of iron oxide in hyperlipidemic rabbits. Circulation 2001;103:415-22.

7 Alam SR, Shah AS, Richards J, et al. Ultrasmall superparamagnetic particles of iron oxide in patients with acute myocardial infarction: early clinical experience. Circ Cardiovasc Imaging 2012;5:559-65.

8 Yilmaz A, Dengler MA, van der Kuip $\mathrm{H}$, et al. Imaging of myocardial infarction using ultrasmall superparamagnetic iron oxide nanoparticles: a human study using a multi-parametric cardiovascular magnetic resonance imaging approach. Eur Heart J 2013;34:462-75.

9 Abdel-Aty H, Zagrosek A, Schulz-Menger J, et al. Delayed enhancement and T2weighted cardiovascular magnetic resonance imaging differentiate acute from chronic myocardial infarction. Circulation 2004;109:2411-6.

10 Kellman P, Aletras AH, Mancini C, et al. T2-prepared SSFP improves diagnostic confidence in edema imaging in acute myocardial infarction compared to turbo spin echo. Magn Reson Med 2007;57:891-7.

11 Thygesen K, Alpert JS, Jaffe AS, et al. Third universal definition of myocardial infarction. Jac 2012;60:1581-98. 
12 Taylor AM, Panting JR, Keegan J, et al. Safety and preliminary findings with the intravascular contrast agent NC100150 injection for MR coronary angiography. J Magn Reson Imaging 1999;9:220-7.

13 Thorek DL, Chen AK, Czupryna J, et al. Superparamagnetic iron oxide nanoparticle probes for molecular imaging. Ann Biomed Eng 2006;34:23-38.

14 Van Beers BE, Sempoux C, Materne R, et al. Biodistribution of ultrasmall iron oxide particles in the rat liver. J Magn Reson Imaging 2001;13:594-9.

15 Lee WW, Marinelli B, van der Laan AM, et al. PET/MRI of inflammation in myocardial infarction. J Am Coll Cardiol 2012;59:153-63.

16 Stirrat CG, Alam SR, MacGillivray TJ, et al. Ferumoxytol-enhanced magnetic resonance imaging methodology and normal values at 1.5 and 3T. J Cardiovasc Magn Reson 2016;18:46.
17 Verhaert D, Thavendiranathan P, Giri S, et al. Direct T2 quantification of myocardial edema in acute ischemic injury. JACC Cardiovasc Imaging 2011;4:269-78.

18 Giri S, Chung YC, Merchant A, et al. T2 quantification for improved detection of myocardial edema. J Cardiovasc Magn Reson 2009;11:56.

19 Dall'Armellina E, Karia N, Lindsay AC, et al. Dynamic changes of edema and late gadolinium enhancement after acute myocardial infarction and their relationship to functional recovery and salvage index. Circ Cardiovasc Imaging 2011;4:228-36.

20 Nilsson JC, Nielsen G, Groenning BA, et al. Sustained postinfarction myocardial oedema in humans visualised by magnetic resonance imaging. Heart 2001;85:639-42.

21 Alam SR, Lewis SC, Zamvar V, et al. Perioperative elafin for ischaemia-reperfusion injury during coronary artery bypass graft surgery: a randomised-controlled trial. Heart 2015;101:1639-45. 


\section{Heart}

\section{Ferumoxytol-enhanced magnetic resonance imaging assessing inflammation after myocardial infarction}

Colin G Stirrat, Shirjel R Alam, Thomas J MacGillivray, Calum D Gray, Marc R Dweck, Jennifer Raftis, William SA Jenkins, William A Wallace, Renzo Pessotto, Kelvin HH Lim, Saeed Mirsadraee, Peter A Henriksen, Scott IK Semple and David E Newby

Heart 2017 103: 1528-1535 originally published online June 22, 2017 doi: 10.1136/heartjnl-2016-311018

Updated information and services can be found at: http://heart.bmj.com/content/103/19/1528

\begin{tabular}{cl} 
References & $\begin{array}{l}\text { These include: } \\
\text { This article cites 21 articles, 13 of which you can access for free at: } \\
\text { http://heart.bmj.com/content/103/19/1528\#BIBL }\end{array}$ \\
Open Access & $\begin{array}{l}\text { This is an Open Access article distributed in accordance with the terms of } \\
\text { the Creative Commons Attribution (CC BY 4.0) license, which permits } \\
\text { others to distribute, remix, adapt and build upon this work, for commercial } \\
\text { use, provided the original work is properly cited. See: } \\
\text { http://creativecommons.org/licenses/by/4.0/ }\end{array}$ \\
$\begin{array}{c}\text { Email alerting } \\
\text { service }\end{array}$ & $\begin{array}{l}\text { Receive free email alerts when new articles cite this article. Sign up in the } \\
\text { box at the top right corner of the online article. }\end{array}$ \\
\hline $\begin{array}{c}\text { Topic } \\
\text { Collections }\end{array}$ & $\begin{array}{c}\text { Articles on similar topics can be found in the following collections } \\
\text { Editor's choice (233) }\end{array}$ \\
\hline
\end{tabular}

\section{Notes}

To request permissions go to:

http://group.bmj.com/group/rights-licensing/permissions

To order reprints go to:

http://journals.bmj.com/cgi/reprintform

To subscribe to BMJ go to:

http://group.bmj.com/subscribe/ 


\title{
(6) OPEN ACCESS
}

\section{Ferumoxytol-enhanced magnetic resonance imaging in acute myocarditis}

\author{
Colin G Stirrat, ${ }^{1}$ Shirjel R Alam, ${ }^{1}$ Thomas J MacGillivray, ${ }^{2,3}$ Calum D Gray, ${ }^{2,3}$ \\ Marc R Dweck, ${ }^{1}$ Kevin Dibb, ${ }^{1}$ Nick Spath, ${ }^{1}$ John R Payne, ${ }^{4}$ Sanjay K Prasad, ${ }^{5}$ \\ Roy S Gardner, ${ }^{4}$ Saeed Mirsadraee, ${ }^{6}$ Peter A Henriksen, ${ }^{1}$ Scott IK Semple, ${ }^{1,3}$ \\ David E Newby ${ }^{1,3}$
}

'British Heart Foundation/ University Centre for Cardiovascular Science, University of Edinburgh, Edinburgh, UK

${ }^{2}$ Clinical Research Facility, University of Edinburgh, Edinburgh, UK

${ }^{3}$ Edinburgh Imaging QMRI

Facility, University of Edinburgh, Edinburgh, UK

${ }^{4}$ Department of Cardiology, Golden Jubilee National Hospital, Clydebank, UK

${ }^{5}$ Department of Cardiology, Royal Brompton Hospital, London, UK

${ }^{6}$ Department of Radiology, Royal Brompton Hospital, London, UK

\section{Correspondence to}

Dr Colin G Stirrat, British Heart Foundation, University Centre for Cardiovascular Science Room SU 305 Chancellor's Building, University of Edinburgh, 49 Little France Crescent, Edinburgh, EH16 4SB UK; colin.stirrat@ed.ac.uk

Received 13 April 2017 Revised 24 May 2017 Accepted 12 June 2017

\section{CLinked}

- http://dx.doi.org/10.1136 heartjnl-2017-312004

CrossMark

To cite: Stirrat CG, Alam SR, MacGillivray TJ, et al. Heart Published Online First: [please include Day Month Year]. doi:10.1136/ heartjnl-2017-311688

\begin{abstract}
Objectives Ultrasmall superparamagnetic particles of iron oxide (USPIO)-enhanced MRI can detect tissueresident macrophage activity and identify cellular inflammation within tissues. We hypothesised that USPIO-enhanced MRI would provide a non-invasive imaging technique that would improve the diagnosis and management of patients with acute myocarditis.

Methods Ten volunteers and 14 patients with suspected acute myocarditis underwent $\mathrm{T} 2, \mathrm{~T} 2^{*}$ and late gadolinium enhancement (LGE) 3T MRI, with further T2* imaging at 24 hours after USPIO (ferumoxytol, $4 \mathrm{mg} / \mathrm{kg}$ ) infusion, at baseline and 3 months. Myocardial oedema and USPIO enhancement were determined within areas of LGE as well as throughout the myocardium.

Results Myocarditis was confirmed in nine of the 14 suspected cases of myocarditis. There was greater myocardial oedema in regions of LGE in patients with myocarditis when compared with healthy volunteer myocardium ( $T 2$ value, $57.1 \pm 5.3$ vs $46.7 \pm 1.6 \mathrm{~ms}$, $p<0.0001)$. There was no demonstrable difference in USPIO enhancement between patients and volunteers even within regions displaying LGE (change in $R 2^{*}, 35.0 \pm 15.0$ vs $37.2 \pm 9.6 \mathrm{~s}^{-1}, \mathrm{p}>0.05$ ). Imaging after 3 months in patients with myocarditis revealed a reduction in volume of $L G E$, a reduction in oedema measures within regions displaying LGE and improvement in ejection fraction (mean $-19.7 \mathrm{~mL}$, $95 \% \mathrm{Cl}(-0.5$ to -40.0$)),-5.8 \mathrm{~ms}(-0.9$ to -10.7$)$ and $+6 \%(0.5 \%$ to $11.5 \%)$, respectively, $\mathrm{p}<0.05$ for all). Conclusion In patients with acute myocarditis, USPIOenhanced MRI does not provide additional clinically relevant information to LGE and T2 mapping MRI. This suggests that tissue-resident macrophages do not provide a substantial contribution to the myocardial inflammation in this condition. Clinical trial registration NCT02319278; Results.
\end{abstract}

\section{INTRODUCTION}

Acute myocarditis comprises a wide clinical spectrum from subclinical disease to severe heart failure and is a major cause of sudden death in young adults. ${ }^{1}$ Pathologically, it is characterised by inflammatory cell infiltration of the myocardium with evidence of myocyte necrosis that is not characteristic of an ischaemic aetiology. ${ }^{2}$ Causes of myocarditis include infections, immune-mediated injury and toxins (such as anthracyclines) although frequently no cause is identified. ${ }^{3}$
A variety of inflammatory cells infiltrate the myocardium during myocarditis. In viral myocarditis, the infiltrate is predominantly lymphocyte rich, but other cells including plasma cells, neutrophils, eosinophils, giant cells and macrophages are also present. ${ }^{24-9}$ Monocytes differentiate into macrophages at sites of myocarditis, suggesting that they play an important role in the injury or repair of the myocardium. ${ }^{10}$

Endomyocardial biopsy is considered the gold standard investigation for diagnosis. However, this is prone to sampling error and is uncommon in routine clinical practice. ${ }^{3}$ Cardiac MRI plays a key role in the diagnosis and shows typical appearances on T2-weighted and late gadolinium enhancement (LGE) imaging according to the Lake Louise Criteria. ${ }^{11}$ More recently, quantitative mapping techniques appear to improve diagnostic accuracy further. ${ }^{12}$

Iron oxide nanoparticles are generating interest as an MRI contrast agent able to detect macrophages, and clinical applications are now emerging. ${ }^{13-21} \quad$ Ultrasmall superparamagnetic particles of iron oxide (USPIO) consist of an iron oxide core surrounded by a carbohydrate or polymer coating. They are small enough to extravasate passively through capillaries, where they are engulfed by tissue-resident macrophages ${ }^{22}$ and are detectable by T2\%-weighted MRI. Thus, USPIO-enhanced MRI can identify tissue-resident macrophage activity and identify cellular inflammation within tissues.

In a promising preclinical study of autoimmune myocarditis, ${ }^{23}$ iron nanoparticles were ingested by inflammatory cells and improved distinction of areas with severe inflammation on MRI compared with conventional T2-weighted and gadolinium-enhanced MRI. We aimed to assess and quantify myocardial USPIO enhancement in acute myocarditis and to correlate enhancement with clinical measures of inflammation and oedema using T2 mapping and LGE MRI and compare these measures with healthy volunteers. ${ }^{24}$ We hypothesised that USPIO-enhanced MRI would detect myocardial macrophage activity in myocarditis and provide a cellular-specific non-invasive imaging technique that may aid and improve patient diagnosis and management. 


\section{METHODS}

Patients were recruited from a single centre as part of an openlabel observational multicentre cohort study (ClinicalTrials.gov (NCT02319278); Results). The study was performed in accordance with the Declaration of Helsinki, the approval of the Scotland A Research Ethics Committee and the written informed consent of all participants. The Medicines and Healthcare products Regulatory Authority of the UK gave Clinical Trial Authorisation for the study (EUDraCT 2013-002336-24).

\section{Study populations}

Adult (>18 years of age) patients with suspected acute myocarditis were recruited into the study. The clinical diagnosis was made by an independent clinical cardiologist based on the history, ECG, serum troponin (ARCHITECT STAT troponin I assay, Abbott Laboratories, Illinois, USA) and other available imaging modalities. The diagnosis of myocarditis was verified from case note review by an independent cardiologist and retained for analysis if the MRI scan (reported and agreed by two independent radiologists) showed imaging features of myocarditis on LGE and/or T2 mapping. ${ }^{11}$ Healthy volunteers had no clinically significant medical history. Exclusion criteria were contraindication to MRI or ferumoxytol infusion, any other inflammatory comorbidity, renal failure (estimated glomerular filtration rate $<30 \mathrm{~mL} / \mathrm{min} / 1.73 \mathrm{~m}^{2}$ ), pregnancy, breast feeding and women of childbearing potential without reliable contraception.

\section{Study protocol}

Patients with suspected myocarditis and healthy volunteers underwent paired MRI scans at baseline, and patients were invited to return for repeat imaging after 3 months.

\section{MRI}

MRI was performed using a MAGNETOM Verio 3T MRI scanner running software V.VB17 (Siemens Healthcare, Erlangen, Germany), with a dedicated cardiac array coil. All images were acquired using ECG-gated breath holds. Routine steady state free precession (TrueFISP) sequences were used to acquire long-axis and short-axis images of the heart. Oedema imaging was conducted using a Siemens T2 mapping based on a prototype T2-prepared TrueFISP acquisition acquiring identical long-axis and short-axis slice positions. Quantitative USPIO imaging was performed in similar slice positions using a prototype $\mathrm{T} 2 \%$-weighted multigradient-echo acquisition using a volumetric shim applied over the entire heart volume. Standard cardiac slice widths $(6 \mathrm{~mm}$ width with $4 \mathrm{~mm}$ gap) and eight echo times (2.1-17.1 ms range) with matrix size of $256 \times 115$ were acquired for T2* maps. The in-plane resolution differed as required for larger or smaller objects; generally, a field of view of $400 \times 300 \mathrm{~mm}$ was used with an in-plane resolution of $2.6 \times 1.6 \mathrm{~mm}$. T2* relaxation times were calculated before and after administration of USPIO.

Immediately after the baseline T2, T2* and TrueFISP cine imaging, breath-held inversion recovery sequences in long-axis and short-axis planes were used to acquire late enhancement images following an intravenous administration of gadolinium contrast medium $(0.1 \mathrm{mmol} / \mathrm{kg}$; Gadovist, Bayer, Germany). Optimal inversion time (TI) was determined on a slice-by-slice basis using standard late-enhancement TI-scout protocols. The inversion-recovery late-enhancement short-axis slices were acquired using similar slice positions as the T2 and T2* imaging.
USPIO

Intravenous infusion of USPIO (ferumoxytol, $4 \mathrm{mg} / \mathrm{kg}$; Rienso, Takeda Italia, Italy) was performed immediately following the baseline magnetic resonance scan over 15 min using a concentration of $2-8 \mathrm{mg} / \mathrm{mL}$, diluted in $0.9 \%$ saline or $5 \%$ dextrose. Haemodynamic monitoring was conducted throughout.

\section{Image analysis}

All T2*-weighted multigradient echo images for each patient were analysed using Circle CVI software (Circle CVI42, Canada). An experimentally determined threshold used in previous work ${ }^{14}$ for the coefficient of determination $\left(r^{2}>0.85\right)$ was used to exclude data that did not have an acceptable exponential decay when signal intensity (SI) was plotted against echo time. Individual images affected by artefact were excluded. The inverse of the mean T2* (R2*) for each regions of interest (ROI) was then calculated to assess the uptake of USPIO, where the higher the value, the greater the USPIO accumulation.

T2 mapping, LGE, ventricular volume and functional analyses were also performed using Circle CVI software. T2 and T2* data were collected immediately prior to USPIO administration. USPIO-enhanced T2* data were collected 24-25 hour following ferumoxytol administration.

\section{ROI selection}

ROI were drawn using the standard 16-segment cardiac model ${ }^{25}$ and panmyocardial values averaged using all 16 segments. As a final method of analysis to focus on inflamed myocardium, regions with contiguous $L G E>1 \mathrm{~cm}^{2}$ on a single short-axis slice were retained and visually coaligned with corresponding T2 and $\mathrm{T} 2 *$ images. These corresponding coaligned regions were then averaged to form $\mathrm{LGE}+\mathrm{T} 2$ and $\mathrm{T} 2$ * regions.

\section{Statistical analysis}

All statistical analysis was performed with GraphPad Prism V.6 (GraphPad Software, San Diego, California, USA). Shapiro-Wilk normality testing was carried out prior to testing. To compare USPIO uptake and myocardial oedema in patients and volunteers, R2* and T2 values were compared using paired and unpaired t-tests, Mann-Whitney and Wilcoxon tests depending on pairing and normality of data. To compare results at 3 months with baseline, a paired t-test was used. Statistical significance was defined as two-sided $\mathrm{p}<0.05$.

\section{RESULTS}

Ten volunteers and 14 patients with suspected myocarditis were recruited. Nine patients had confirmed myocarditis according to imaging criteria and independent review. Baseline imaging was conducted within a week of diagnosis. Four patients had alternative diagnoses (takotsubo cardiomyopathy $(\mathrm{n}=2)$, lung cancer $(n=1)$, polymyositis $(n=1))$ and one patient had an incidental finding of an unknown chest wall metallic implant. These five patients were excluded from further analysis. All nine patients retained for analysis had typical features of myocarditis on LGE imaging. Of these nine patients, one patient did not return at baseline for the 24 hours post-USPIO scan. Seven of the nine patients returned at 3 months for repeat imaging.

The healthy volunteer group was older with greater ejection fraction than the myocarditis group at baseline $(p<0.001$ for both, table 1). Patients with myocarditis had greater neutrophil count, $\mathrm{C}$-reactive protein (CRP) and serum troponin concentrations than volunteers $(p<0.05, p<0.01$ and $p<0.0001$, respectively). 


\begin{tabular}{|c|c|c|c|}
\hline & $\begin{array}{l}\text { Healthy } \\
\text { volunteers }\end{array}$ & $\begin{array}{l}\text { Myocarditis } \\
\text { (baseline) }\end{array}$ & $\begin{array}{l}\text { Myocarditis } \\
\text { (3 months) }\end{array}$ \\
\hline Number & 10 & 9 & 7 \\
\hline Female & 6 & 1 & 1 \\
\hline Age (years) & $50(45-53)$ & $28(24-34)^{* * *}$ & $25(23-35)$ \\
\hline Body mass index $\left(\mathrm{kg} / \mathrm{m}^{2}\right)$ & $26(23-29)$ & $25(22-29)$ & $25(22-27)$ \\
\hline $\begin{array}{l}\text { Left ventricular ejection } \\
\text { fraction (\%) }\end{array}$ & $61.1 \pm 4.1$ & $51.0 \pm 4.9^{* * *}$ & $57.1 \pm 4.3$ \\
\hline $\begin{array}{l}\text { Late gadolinium } \\
\text { enhancement (mL) }\end{array}$ & Nil & $30.3 \pm 19.3$ & $16.3 \pm 12.3$ \\
\hline ECG & $\mathrm{n} / \mathrm{a}$ & 9/9 & \\
\hline ST elevation & & 7 & \\
\hline T-wave inversion & & 1 & \\
\hline Normal & & 1 & \\
\hline Coronary angiogram & n/a & $4 / 9$ & \\
\hline Normal & & 4 & \\
\hline Echo & n/a & $8 / 9$ & \\
\hline $\begin{array}{l}\text { Wall motion } \\
\text { abnormality }\end{array}$ & & 4 & \\
\hline Normal & & 4 & \\
\hline \multicolumn{4}{|l|}{ Blood tests } \\
\hline $\begin{array}{l}\text { White cell count } \\
\left(\times 10^{9} / \mathrm{L}\right)\end{array}$ & $5.8(4.2-7.1)$ & $7.1(6.1-8.9)$ & $5.1(4.6-5.6)$ \\
\hline $\begin{array}{l}\text { Neutrophil count } \\
\left(\times 10^{9} / L\right)\end{array}$ & $3.2(2.2-4.2)$ & $4.4(3.7-5.2)^{*}$ & $2.8(2.7-3.5)$ \\
\hline $\begin{array}{l}\text { Lymphocyte count } \\
\left(\times 10^{9} / \mathrm{L}\right)\end{array}$ & $1.7(1.4-2.1)$ & $1.8(1.3-2.2)$ & $1.8(1.2-1.9)$ \\
\hline $\begin{array}{l}\text { Monocyte count } \\
\left(\times 10^{9} / \mathrm{L}\right)\end{array}$ & $0.4(0.3-0.7)$ & $0.6(0.5-0.8)$ & $0.4(0.3-0.5)$ \\
\hline $\begin{array}{l}\text { C-reactive protein } \\
(\mathrm{mg} / \mathrm{L})\end{array}$ & $1.0(1.0-2.5)$ & $38(14-63)^{* *}$ & $2.0(1.0-2.0)$ \\
\hline Troponin (ng/L) & $2.0\{1.5-4.0)$ & $\begin{array}{l}121842(3950- \\
25722)^{* * * *}\end{array}$ & $4.0(2.0-6.0)$ \\
\hline
\end{tabular}

Mean \pm SD or median (IQR).

${ }^{*} \mathrm{p}<0.05 ;{ }^{* *} \mathrm{p}<0.01 ;{ }^{* * *} \mathrm{p}<0.001 ;{ }^{* * *} \mathrm{p}<0.0001$ (compared with volunteers at baseline).

There were no other significant differences between participant groups at baseline.

Administration of ferumoxytol was well tolerated with no adverse reactions reported during or immediately after administration in any of the participants.

\section{USPIO enhancement}

At baseline, there were no differences in R2* values between healthy volunteers and patients with myocarditis (panmyocardium and LGE + regions; Figures 1, 2). After USPIO administration, the R2* in all three groups increased but there was no difference in either the change in or post-USPIO, R2* between the groups (figure 2 and table 2).

\section{Myocardial oedema}

There was no difference in panmyocardial T2 between volunteers and patients with myocarditis. Regions displaying LGE in myocarditis patients had higher T2 than panmyocardial values for both healthy volunteers and patients with myocarditis $(\mathrm{p}<0.0001$ and $\mathrm{p}<0.01$, respectively; figure 2 and table 2$)$.

\section{Baseline versus 3-month imaging in patients with myocarditis}

There were no changes in myocardial USPIO uptake between baseline and 3 months either on panmyocardial analysis
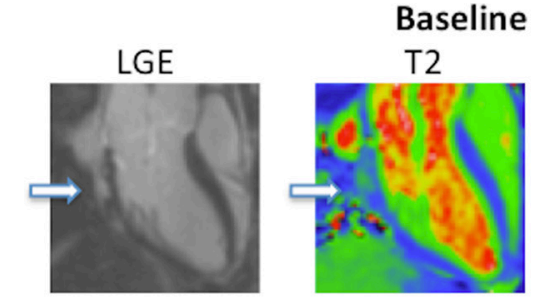

24h Post USPIO R2*
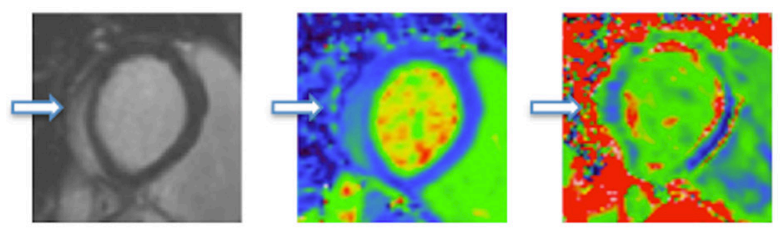

3months
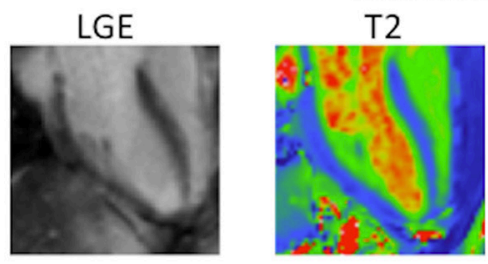

24h Post USPIO R2*
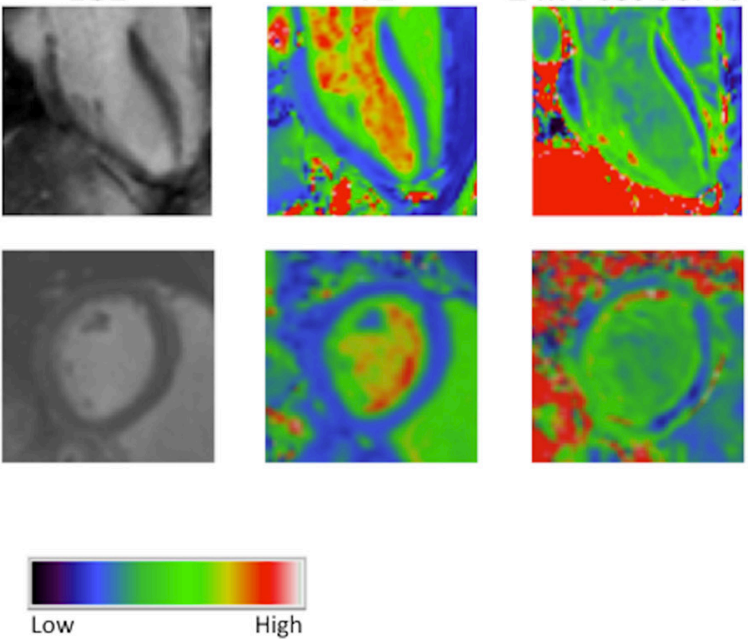

Figure 1 Images of patient with myocarditis. Three chamber and basal short-axis images of a patient with myocarditis displaying patchy posterolateral late gadolinium enhancement (LGE) (white regions, arrowed) that correspond to oedematous regions of myocardium on the T2 map (lighter regions, arrowed). There is no uptake of ultrasmall superparamagnetic particles of iron oxide (USPIO) in the post-USPIO R2* maps; corresponding regions may even have lower R2* in this patient. Myocardial oedema appears to normalise but subtle LGE remains at 3 months.

(mean $+7.6 \mathrm{~s}^{-1} 95 \% \mathrm{CI}(-4.8$ to 19.9$\left.)\right)$ or in regions with LGE $\left(-4.2 \mathrm{~s}^{-1}\right.$ ( -21.9 to 13.5$), \mathrm{p}>0.05$ for both; figure 3$)$. Panmyocardial T2 did not change over 3 months ( -1.1 (-5.8 to 3.5), $\mathrm{p}>0.05)$. Volume of LGE and T2 within regions displaying LGE reduced over the 3 -month period $(-19.7 \mathrm{~mL}(-0.5$ to -40.0$)$ and $-5.8 \mathrm{~ms}(-0.9$ to -10.7$)$, respectively, $\mathrm{p}<0.05$ for both). Ejection fraction increased over the 3 -month period $(+6 \%(0.5$ to 11.5$), \mathrm{p}<0.05)$.

\section{DISCUSSION}

For the first time, we report the combined assessment of myocarditis using LGE, T2 mapping and USPIO-enhanced T2* MRI. USPIO-enhanced T2* MRI has previously been used in man to assess cardiovascular inflammation in a range of different conditions, ${ }^{14} 162126$ with preliminary positive results from a rodent model of myocarditis. This raised hope that USPIO-enhanced T2* MRI might add useful cell-specific clinical information in patients with myocarditis. However, while we found typical features of myocarditis using standard imaging with LGE and 


\section{Myocardial USPIO enhancement}

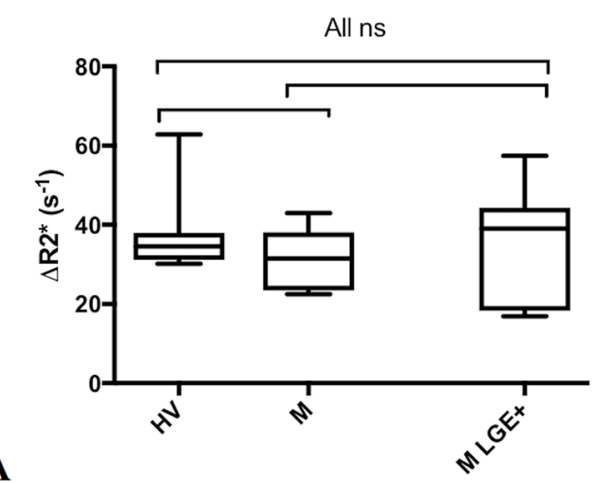

Myocardial Oedema

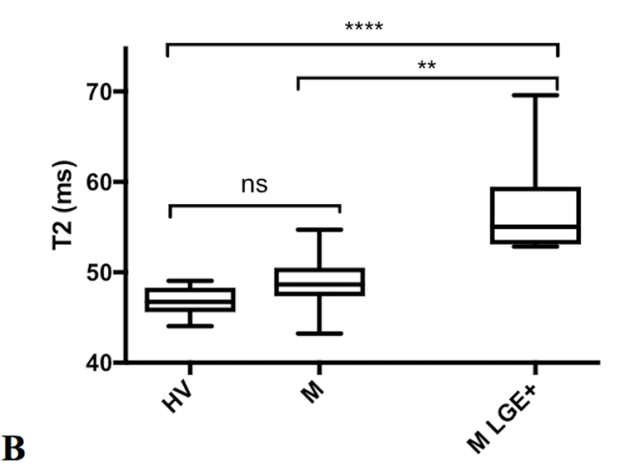

Figure 2 Myocardial USPIO enhancement vs Oedema at Baseline. Changes in myocardial R2* due to USPIO accumulation (A, left) and myocardial oedema by $\mathrm{T} 2$ mapping $(\mathrm{B}$, right) are shown in healthy volunteers $(\mathrm{HV})$ and patients with myocarditis $(\mathrm{M})$ using panmyocardial average, and further limited to regions displaying LGE in patients with myocarditis (M LGE+). There were no significant differences in $\triangle R 2{ }^{*}$ due to USPIO accumulation between all groups ( $p>0.05$ for all). There was no difference in panmyocardial T2 between volunteers with patients with myocarditis. Myocardial regions displaying LGE (M LGE+) had greater T2 than the panmyocardial value for healthy volunteers and myocarditis patients $\left({ }^{* * *} p<0.0001\right.$ and ${ }^{* *} \mathrm{p}<0.01$ respectively).

T2 mapping cardiovascular magnetic resonance (CMR), we did not detect USPIO enhancement within the myocardium of our patients. This suggests that USPIO-enhanced MRI provides no additional diagnostic value in these patients and that tissue-resident macrophages are not a major contributor to the cellular inflammation following acute myocarditis.

Our case study population of nine patients were excellent examples of myocarditis. They were young, with significantly elevated levels of systemic inflammatory markers, including neutrophil count, CRP and cardiac-specific high-sensitivity troponin, and all had typical features of myocarditis on CMR reported by two independent clinical radiologists. Most patients had ECG changes suggesting significant myocyte injury and several had normal coronary angiograms. All patients that did not have a coronary angiogram did not have any risk factors for coronary artery disease. We detected MRI features of myocarditis in every patient. LGE was discontinuous in nature and generally epicardial and subepicardial in distribution, but could be found in the midwall and occasionally in the subendocardium. LGE was never found in the subendocardium alone or confined to one coronary territory that would be more suggestive of myocardial infarction. In keeping with the typical distribution of myocarditis, most patients displayed LGE in the inferior, posterior and lateral walls. We are therefore confident that the diagnosis of myocarditis was robust in all patients, and an independent cardiologist verified this.

Table 2 Myocardial R2* and T2 values in healthy volunteers and patients with myocarditis

\begin{tabular}{llll}
\hline & $\begin{array}{l}\text { Healthy volunteers } \\
\text { (panmyocardial) }\end{array}$ & $\begin{array}{l}\text { Myocarditis } \\
\text { (panmyocardial) }\end{array}$ & $\begin{array}{l}\text { Myocarditis } \\
\text { (LGE+ regions) }\end{array}$ \\
\hline $\begin{array}{l}\text { Panmyocardial pre- } \\
\text { USPIO R2* } \\
\left(\mathrm{s}^{-1}\right)\end{array}$ & $46.9 \pm 4.1$ & $43.7 \pm 5.3$ & $51.8 \pm 8.3$ \\
$\begin{array}{l}\text { Panmyocardial post- } \\
\text { USPIO R2* } \\
\left(\mathrm{s}^{-1}\right)\end{array}$ & $84.2 \pm 12.4$ & $75.6 \pm 11.3$ & $86.1 \pm 15.3$ \\
$\begin{array}{l}\text { Panmyocardial } \\
\triangle R 2{ }^{*}\left(\mathrm{~s}^{-1}\right)\end{array}$ & $37.2 \pm 9.6$ & $31.4 \pm 7.5$ & $35.0 \pm 15.0$ \\
$\mathrm{~T} 2(\mathrm{~ms})$ & $46.7 \pm 1.6$ & $48.9 \pm 3.1$ & $57.1 \pm 5.3^{* * * *}$ \\
\hline
\end{tabular}

Mean \pm SD.

$* * * * p<0.0001$ (compared with volunteers).
Myocardial oedema was easily visualised, and indeed quantified, using T2 mapping (Figure 2). We found intense myocardial oedema in our myocarditis cohort with maximal T2 values approaching $70 \mathrm{~ms}$ on $\mathrm{T} 2$ mapping. Within regions displaying LGE $(\mathrm{LGE}+)$, there was a profound increase in T2 when compared with volunteers. This signal was so powerful that a trend remained evident when comparing panmyocardial T2 values to those of volunteers, although the difference was not statistically significant. Seven patients returned for repeat imaging assessment at 3 months, where we recorded an improvement in clinical features: a reduction in the volume of LGE (by around 50\%), a reduction in oedema within inflamed (LGE+) regions and an overall improvement in ejection fraction.

We were unable to detect USPIO accumulation in our cohort of patients with myocarditis. We know that macrophages can be present in myocarditis, although they are generally not the predominant cell type. It is possible that patients within our group had predominantly lymphocytic or neutrophil-rich cell infiltrate and the finding of greater levels of neutrophils in patients with myocarditis at baseline may support the latter. The clinical care team did not feel that endomyocardial biopsy was justified in any of the cases, and lack of histological data is clearly a limitation. In recent work, we have detected USPIO-laden macrophages in patients with recent myocardial infarction and demonstrated macrophage uptake of USPIOs in biopsies taken at the time of cardiac surgery. ${ }^{13}$ We therefore acknowledge that while some USPIO-laden macrophages may have been present in patients with myocarditis, there were either insufficient numbers of macrophages or USPIO engulfment was inhibited or deficient. Ultimately, USPIO-enhanced T2* CMR was unable to demonstrate increased macrophage activity and lacked sensitivity for detecting myocarditis.

A further contributory reason for failure to detect USPIO enhancement within inflamed regions may be due to difficulties encountered in USPIO-enhanced T2* imaging. These are discussed in greater detail elsewhere, ${ }^{24}$ but artefact due to breathing and in particular, 'blooming' artefacts from the vascular organs of the lungs, liver and stomach can be problematic. This can make analysis more challenging by having to exclude the affected later echo times from T2* decay curve fitting. Indeed, 'blooming' artefacts from nearby tissues and organs most commonly affect the inferior and lateral walls, 
Change in $\mathrm{R2}^{*}$ - panmyocardium

A

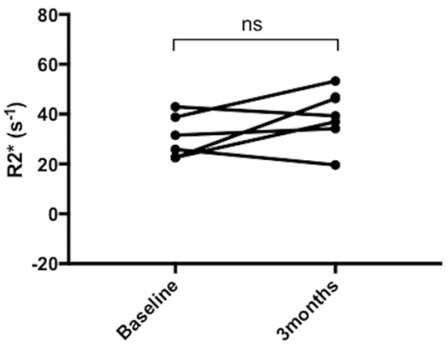

Change in T2 - LGE+

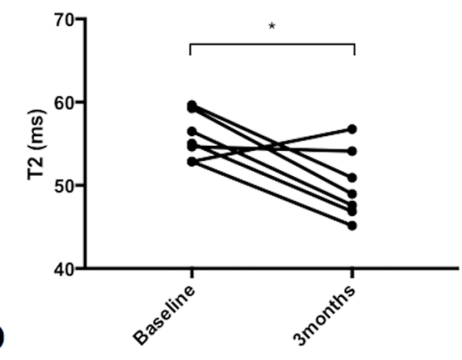

Change in R2* LGE+

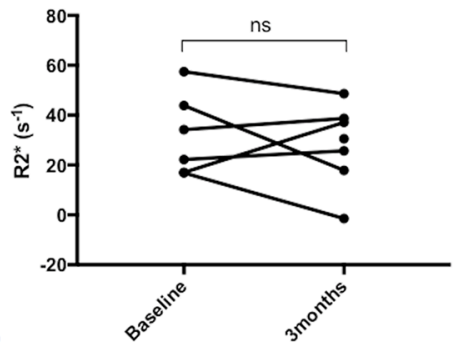

Change in LGE

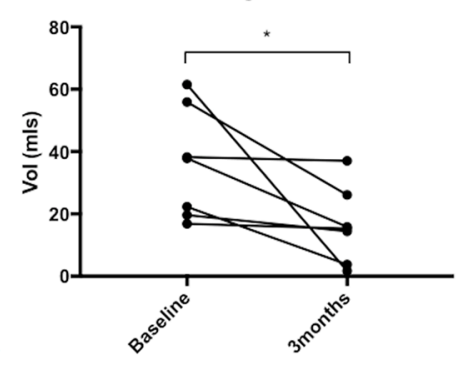

Change in T2 - panmyocardium

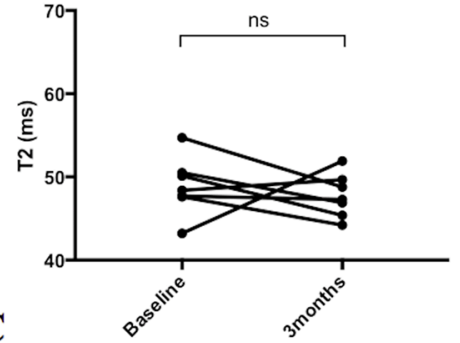

Change in EF

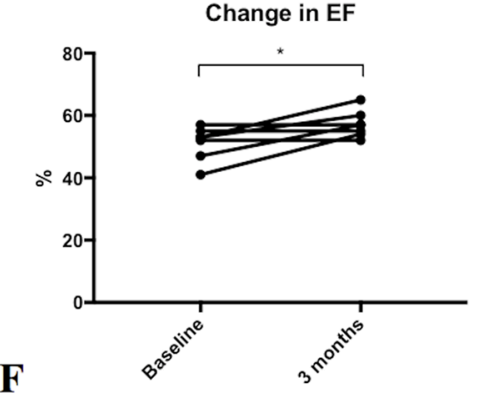

Figure 3 Changes on repeat imaging at 3 months in patients with myocarditis. There was no significant (ns) difference in ultrasmall superparamagnetic particles of iron oxide uptake between baseline and 3 months in both panmyocardium $(A)$ and in regions displaying late gadolinium enhancement (LGE) (B). There was no difference in panmyocardial T2 (C) over 3 months. T2 in regions displaying LGE (D) reduced, as did the volume of LGE (E) over the 3-month period. There was an increase in ejection fraction (EF) between baseline and 3 months (F); ${ }^{*} p<0.05$.

which is the usual site of inflammation in myocarditis. Therefore, the myocardial regions of greatest interest were often the most difficult to analyse although this can usually be overcome. ${ }^{24}$ This may go some way to explain why there is greater spread of R2* data in patients with myocarditis, especially in LGE+ regions. Finally, patients with myocarditis often had symptoms of pleurisy and pericarditis, and despite adequate analgesia, often found the long breath holding required for T2* imaging difficult.

Some final limitations also deserve mention. First, the sample size was small, but numbers in this pilot study were sufficient to show that USPIO-enhanced T2* CMR fails to add clinically relevant information to CMR imaging parameters of LGE and T2 mapping for individual patients. Second, the control volunteer group was not age or sex matched but we have no reason to believe that this influenced the results. Lastly, myocardial

\section{Key messages}

\section{What is already known about this subject?}

Ultrasmall superparamagnetic particles of iron oxide (USPIO) are ingested by tissue macrophages that can be visualised using MRI to highlight areas of inflammation within the heart.

\section{What does this study add?}

USPIO-enhanced T2* MRI does not provide additional clinically relevant information to that of LGE and T2 mapping MRI. This suggests that tissue-resident macrophages do not provide a substantial contribution to the myocardial inflammation in this condition.

\section{How might this impact on clinical practice?}

USPIO-enhanced T2* MRI may still prove to be of value in diagnosing and monitoring conditions with macrophagedriven myocardial inflammation, but its role in assessing acute myocarditis appears limited. geometry is affected by several factors that vary between scans such as the presence of tissue oedema, heart rate and blood volume status and accurate coregistration can be challenging.

Despite these results, USPIO-enhanced imaging still holds promise as a non-invasive imaging method for the diagnosis and monitoring of tissue inflammatory macrophages in the heart. We have recently found that USPIO-enhanced MRI can detect and serially monitor macrophages after myocardial infarction $(\mathrm{MI})^{13}$ and work is under way at our centre as part of this project (NCT02319278) to assess the value of this technique in diagnosing cardiac sarcoidosis and cardiac transplant rejection. If successful, USPIO-enhanced MRI may provide a platform on which to assess existing and novel therapeutic interventions that might modify the inflammatory process, not only after MI but also in other inflammatory processes affecting the heart.

In conclusion, we have shown that in patients with acute myocarditis, USPIO-enhanced T2* MRI does not provide additional clinically relevant information to that of LGE and T2 mapping MRI. This suggests that tissue-resident macrophages do not provide a substantial contribution to the myocardial inflammation in this condition.

Acknowledgements This work was supported by the Chief Scientist Office (ETM/266). SRA and DEN are supported by the British Heart Foundation (FS/12/83; $\mathrm{CH} / 09 / 002)$. DEN is the recipient of a Wellcome Trust Senior Investigator Award (WT103782AIA). Wellcome Trust Clinical Research Facility and the Clinical Research Imaging Centre are supported by NHS Research Scotland (NRS) through NHS Lothian.

Contributors CGS, SRA, SIKS and DEN designed the study, collected and analysed the data and drafted the manuscript. TJM, CDG, KD and NS analysed and interpreted the data and drafted the manuscript. MRD, JRP, SKP, RSG, SM and PAH designed the study and drafted the manuscript. All authors read and approved the manuscript. CGS is responsible for overall content as guarantor.

Funding This work was supported by the Chief Scientist Office (ETM/266).

Competing interests None declared.

Patient consent Obtained.

Ethics approval Scotland A Research Ethics Committee. 
Provenance and peer review Not commissioned; externally peer reviewed.

Open Access This is an Open Access article distributed in accordance with the terms of the Creative Commons Attribution (CC BY 4.0) license, which permits others to distribute, remix, adapt and build upon this work, for commercial use, provided the original work is properly cited. See: http://creativecommons.org/ licenses/by/4.0/

(C) Article author(s) (or their employer(s) unless otherwise stated in the text of the article) 2017. All rights reserved. No commercial use is permitted unless otherwise expressly granted.

\section{REFERENCES}

1 Drory $Y$, Turetz $Y$, Hiss $Y$, et al. Sudden unexpected death in persons less than 40 years of age. Am J Cardio/ 1991;68:1388-92.

2 Aretz HT, Billingham ME, Edwards WD, et al. Myocarditis. A histopathologic definition and classification. Am J Cardiovasc Pathol 1987;1:3-14.

3 Caforio ALP, Pankuweit S, Arbustini E, et al. Current state of knowledge on aetiology, diagnosis, management, and therapy of myocarditis: a position statement of the European Society of Cardiology Working Group on Myocardial and Pericardial diseases: The Oxford University Press, 2013:2636-48.

4 Magnani JW, Dec GW. Myocarditis: current trends in diagnosis and treatment. Circulation 2006:113:876-90.

5 Sagar S, Liu PP, Cooper LT. Myocarditis. Lancet 2012;379:738-47.

6 Barin JG, Rose NR, Ciháková D. Macrophage diversity in cardiac inflammation: a review. Immunobiology 2012;217:468-75.

7 Jaquenod De Giusti C, Ure AE, Rivadeneyra L, et al. Macrophages and galectin 3 play critical roles in CVB3-induced murine acute myocarditis and chronic fibrosis. J Mol Cell Cardiol 2015:85:58-70.

8 Feldman AM, McNamara D. Myocarditis. N Engl J Med 2000;343:1388-98.

9 Li K, Xu W, Guo Q, et al. Differential macrophage polarization in male and female $B A L B / c$ mice infected with coxsackievirus B3 defines susceptibility to viral myocarditis. Circ Res 2009;105:353-64.

10 Baba T, Ishizu A, Iwasaki S, et al. CD4+/CD8+ macrophages infiltrating at inflammatory sites: a population of monocytes/macrophages with a cytotoxic phenotype. Blood 2006;107:2004-12.

11 Friedrich MG, Sechtem U, Schulz-Menger J, et a/Cardiovascular magnetic resonance in myocarditis: a JACC white paperJ Am Coll Cardiol. , 2009:87, 1475. doi.

12 Luetkens JA, Homsi R, Sprinkart AM, et al. Incremental value of quantitative CMR including parametric mapping for the diagnosis of acute myocarditis. Eur Heart $J$ Cardiovasc Imaging 2016:17:154-61.

13 Stirrat C, Alam S, MacGillivray T, et al. MRI enhanced with ultrasmall superparamagnetic particles of iron oxide in the assessment of cellular inflammation after myocardial infarction. Lancet 2016:387:594.
14 Alam SR, Shah AS, Richards J, et al. Ultrasmall superparamagnetic particles of iron oxide in patients with acute myocardial infarction: early clinical experience. Circ Cardiovasc Imaging 2012;5:559-65.

15 Yilmaz A, Dengler MA, van der Kuip $\mathrm{H}$, et al. Imaging of myocardial infarction using ultrasmall superparamagnetic iron oxide nanoparticles: a human study using a multi-parametric cardiovascular magnetic resonance imaging approach. Eur Heart $\rfloor$ 2013:34:462-75.

16 Richards JM, Semple SI, MacGillivray TJ, et al. Abdominal aortic aneurysm growth predicted by uptake of ultrasmall superparamagnetic particles of iron oxide: a pilot study. Circ Cardiovasc Imaging 2011;4:274-81.

17 McBride OM, Berry C, Burns P, et al. MRI using ultrasmall superparamagnetic particles of iron oxide in patients under surveillance for abdominal aortic aneurysms to predict rupture or surgical repair: MRI for abdominal aortic aneurysms to predict rupture or surgery-the MA(3)RS study. Open Heart 2015;2:e000190-0.

18 Trivedi RA, Mallawarachi C, U-King-Im JM, et al. Identifying inflamed carotid plaques using in vivo USPIO-enhanced MR imaging to label plaque macrophages. Arterioscler Thromb Vasc Bio/ 2006;26:1601-6.

19 Trivedi RA, U-King-Im JM, Graves MJ, et al. In vivo detection of macrophages in human carotid atheroma: temporal dependence of ultrasmall superparamagnetic particles of iron oxide-enhanced MRI. Stroke 2004;35:1631-5.

20 Tang T, Howarth SP, Miller SR, et al. Assessment of inflammatory burden contralateral to the symptomatic carotid stenosis using high-resolution ultrasmall, superparamagnetic iron oxide-enhanced MRI. Stroke 2006:37:2266-70.

21 Tang TY, Howarth SP, Miller SR, et al. The ATHEROMA (atorvastatin therapy: effects on reduction of macrophage activity) study. Evaluation using ultrasmall superparamagnetic iron oxide-enhanced magnetic resonance imaging in carotid disease. J Am Coll Cardiol 2009;53:2039-50.

22 Ruehm SG, Corot $\mathrm{C}$, Vogt $\mathrm{P}$, et al. Magnetic resonance imaging of atherosclerotic plaque with ultrasmall superparamagnetic particles of iron oxide in hyperlipidemic rabbits. Circulation 2001;103:415-22.

23 Moon H, Park HE, Kang J, et al. Noninvasive assessment of myocardial inflammation by cardiovascular magnetic resonance in a rat model of experimental autoimmune myocarditis. Circulation 2012;125:2603-12.

24 Stirrat CG, Alam SR, MacGillivray TJ, et al. Ferumoxytol-enhanced magnetic resonance imaging methodology and normal values at 1.5 and 3T. J Cardiovasc Magn Reson 2016;18:46.

25 Cerqueira MD, Weissman NJ, Dilsizian V, et al. Standardized myocardial segmentation and nomenclature for tomographic imaging of the heart: astatement for healthcare professionals from the cardiac Imaging Committee of the Council on clinical cardiology of the American Heart Association. Journal of the American Society of Echocardiography 2002;15:463-7.

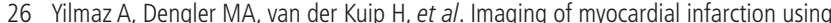
ultrasmall superparamagnetic iron oxide nanoparticles: a human study using a multi-parametric cardiovascular magnetic resonance imaging approach. Eur Heart $\rfloor$ 2013;34:462-75. 